\title{
Phytodiversität, Charakterisierung und Syntaxonomie der Trockenrasen auf Saaremaa (Estland)
}

\author{
Diplomarbeit im Studiengang Umweltwissenschaften \\ vorgelegt von Steffen Boch \\ Goethestraße 12 b \\ 21335 Lüneburg \\ Matrikelnummer: 111410
}

Institut für Ökologie und Umweltchemie

Universität Lüneburg

Lüneburg, den 15.11.2005 


\section{Inhaltsverzeichnis}

$1 \quad$ Einleitung.............................................................................................................

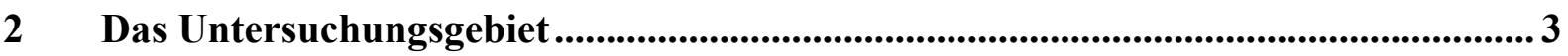

2.1 Lage und allgemeine Gebietsbeschreibung .............................................................. 3

2.2 Geologie und Geomorphologie ................................................................................. 4

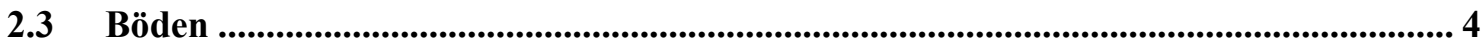

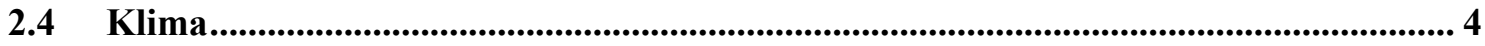

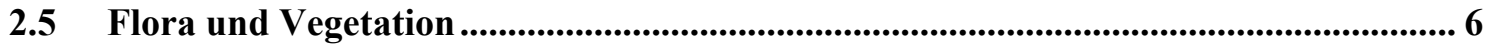

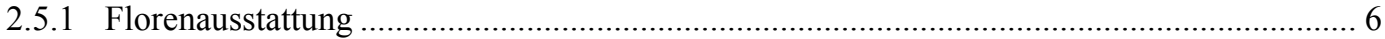

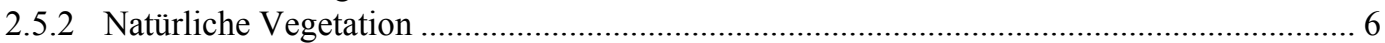

2.5.3 Vegetationsgeschichte und anthropogene Einflüsse ................................................... 8

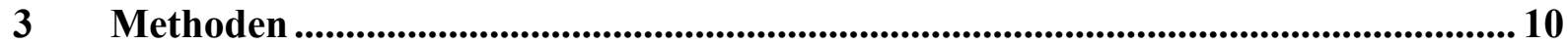

3.1 Aufnahmeflächen und Erhebungen im Gelände.............................................................. 10

3.1.1 Verteilung und Auswahl der Aufnahmeflächen............................................................... 10

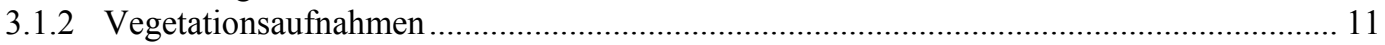

3.2 Sippennomenklatur und Bestimmungshilfen ....................................................... 15

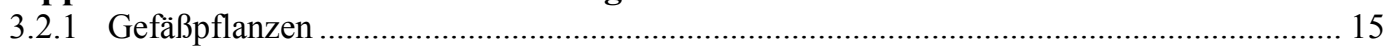

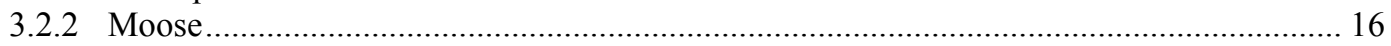

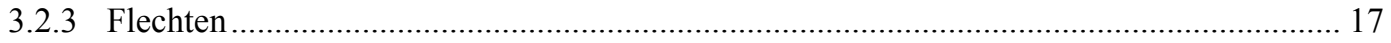

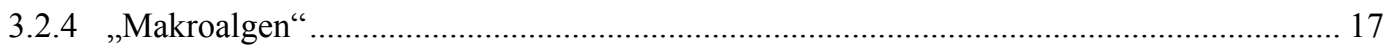

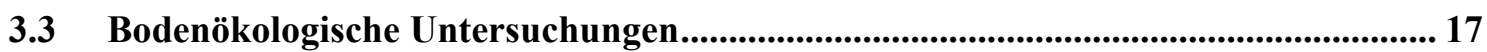

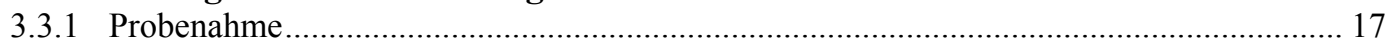

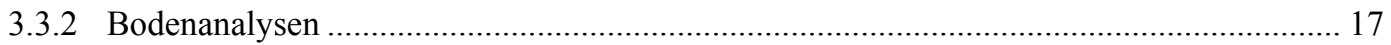

3.4 Syntaxonomische Methoden ......................................................................................... 19

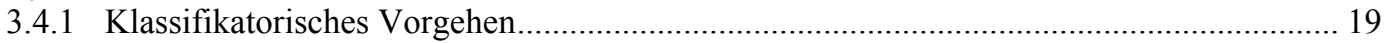

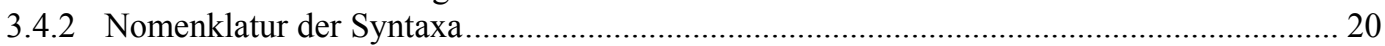

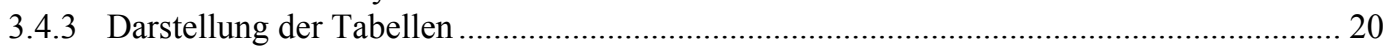

3.4.4 Diagnostische Artenkombination einer Gesellschaft ............................................... 21

3.5 Statistische Auswertung .......................................................................................................... 21

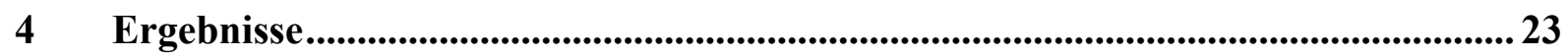

4.1 Charakterisierung der Pflanzengesellschaften..................................................................... 23

4.1.1 Caricetum arenariae Christiansen 1927 ....................................................................... 23

4.1.2 Helichryso arenarii-Jasionetum litoralis Libbert 1940 .................................................... 24

4.1.3 Festucetum polesicae Regel 1928 ............................................................................... 26

4.1.4 Sileno otitae-Festucetum brevipilae Libbert 1933 corr. Kratzert \& Dengler 1999 …......... 28

4.1.5 Cladonio symphycarpiae-Sedetum albi Tx. 1951 ........................................................... 30

4.1.6 Crepido pumilae-Allietum alvarensis Krahulec et al. ex Dengler \& Löbel 2006 ................ 31

4.1.7 Helictotrichon pratense-[Brachypodietalia pinnati]-Gesellschaft........................................ 33

4.2 Ökologie und Phytodiversität - eine vergleichende Betrachtung der Trockenrasengesellschaften Saaremaas ............................................................................................................... 34

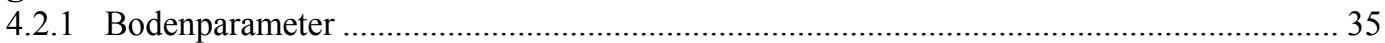

4.2.2 Deckungsgrade der Vegetation und andere Strukturparameter...……………………….... 39

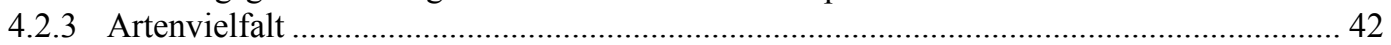

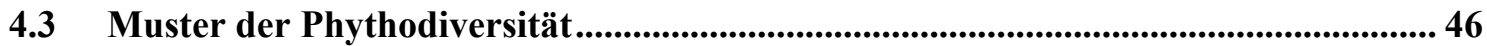

4.3.1 Beziehungen zwischen Artenreichtum und abiotischen Umweltparametern ...................... 46

4.3.2 Beziehungen zwischen Artenreichtum und biotischen Umweltparametern .......................... 50 


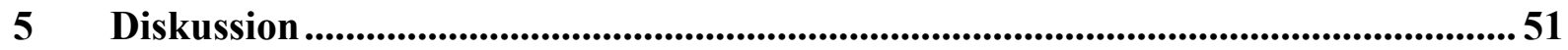

5.1 Klassifikation und Charakterisierung der estnischen Trockenrasen im überregionalen Vergleich

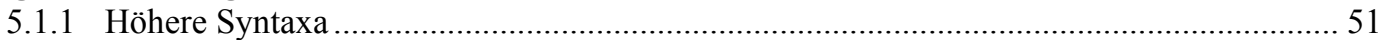

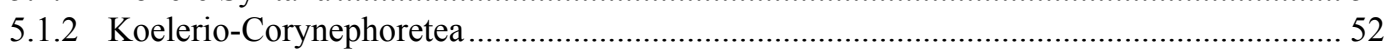

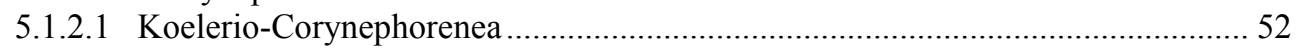

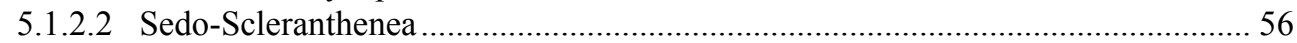

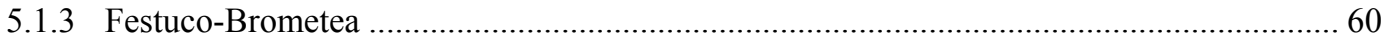

5.1.4 Zusammenfassung der syntaxonomischen Klassifikation...........................................62

5.2 FFH-Schutzstatus der Trockenrasengesellschaften des Untersuchungsgebietes ...... 63

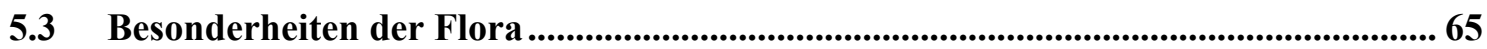

$5.4 \quad$ Ursachen der Phytodiversitätsmuster.......................................................................................68 68

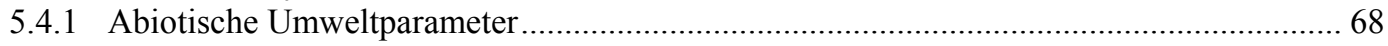

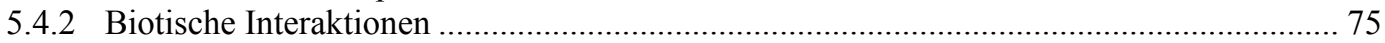

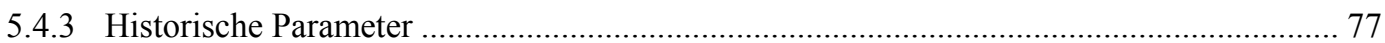

5.4.4 Zusammenfassung der Phytodiversitätsmuster .................................................................. 77

Zusammenfassung ............................................................................................................................. 79

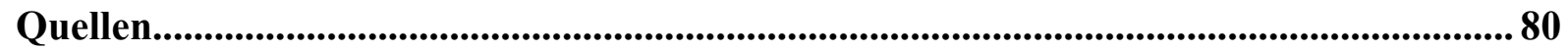

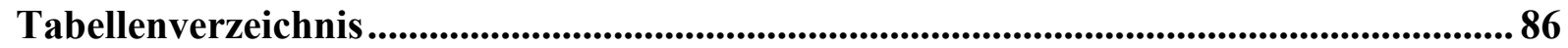

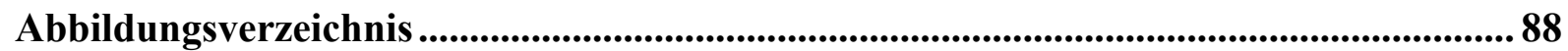

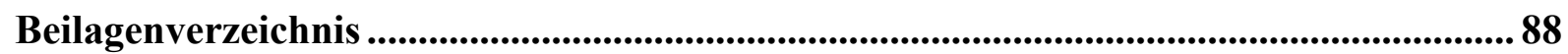

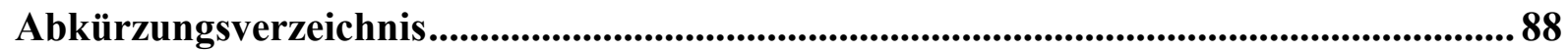

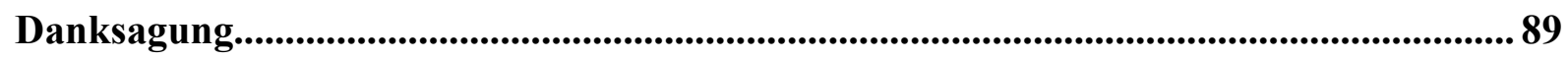

Anhang 1: Verzeichnis der Pflanzensippen .........................................................................

Anhang 2: GPS-Daten der Vegetationsaufnahmen (UTM WGS-84) ............................. VII 


\section{Einleitung}

Trockenrasen zählen zu den artenreichsten Vegetationstypen Europas. Dies gilt sowohl bezogen auf das gesamte Arteninventar der Vegetationstypen (z. B. PÄRTEL et al. 1996, 1999a, KORNECK et al. 1998) als auch für die Artendichten auf kleineren Flächengrößen (z. B. HOBOHм 1998, PÄRTEL et al. 1999a, DENGLER 2005). Sie sind jedoch aufgrund irreversibler Standortzerstörungen (z. B. Baumaßnahmen), Aufforstung, sowie Nutzungsintensivierung (z. B. intensive Beweidung, Umwandlung von Grün- in Ackerland, Dünger- und Herbizideinsatz) oder -aufgabe mit Verbuschung als Folge überregional im Rückgang begriffen (z. B. KORNECK et al. 1998, POSCHLOD \& SCHUMACHER 1998), so auch in Estland (PAAL 1998, HELM et al. im Druck). Zahlreiche Trockenrasenarten mussten daher bereits in die nationalen Roten Listen aufgenommen werden und stellen in diesen häufig den größten Anteil aller gefährdeten Arten (KORNECK et al. 1998).

Auf der estnischen Insel Saaremaa kommen verschiedene Trockenrasentypen (KoelerioCorynephoretea und Festuco-Brometea) über oberflächennah anstehenden Festgesteinplateaus (Alvare), auf weichseleiszeitlichen Moränen und in den Dünengebieten in Küstennähe noch großflächig vor. Habitatverlust sowie Fragmentierung der Lebensräume sind jedoch auch dort bereits zu einem Problem geworden und stellen langfristig gesehen eine Gefährdung der Phytodiversität in den Trockenrasen dar (z. B. HELM et al. im Druck). Aus naturschutzfachlicher Sicht sind diese Vegetationstypen folglich von besonderem Interesse. Eine wichtige Grundlage zum Schutz und der Erhaltung dieser überwiegend anthropozoogenen Vegetationstypen ist die Kenntnis über deren Vorkommen und Verbreitung.

Zum Zweck der Unterschutzstellung von Vegetationstypen sowie der Abschätzung von Schutzprioritäten werden in der Naturschutzpraxis pflanzensoziologisch möglichst genau definierte Einheiten benötigt. Diese dienen als Grundlage für naturschutzrechtliche Einordnungen und wissenschaftliche Fachgutachten. So werden beispielsweise im Anhang I der Flora-Fauna-Habitat-Richtlinie (FFH-Richtlinie) der Europäischen Union „natürliche Lebensraumtypen von gemeinschaftlichem Interesse, für deren Erhaltung besondere Schutzgebiete ausgewiesen werden müssen“ (EUROPEAN COMMISSION 2003a) beschrieben. Die meisten Trockenrasentypen sind durch diese Richtlinie geschützt, einige sogar prioritär. Des Weiteren ist eine genaue Definition von Vegetationstypen als essenziell für die Erstellung von nationalen Roten Listen der Pflanzengesellschaften anzusehen. Im Hinblick auf zukünftige Maßnahmen zum Erhalt der Trockenrasen auf Saaremaa ist deshalb ein besonderes Augenmerk auf die Synsystematik zu legen.

Über die estnische Trockenrasenvegetation liegen zwar einige auf regionaler Ebene durchgeführte pflanzensoziologische Arbeiten vor (z. B. VILBERG 1927, LIPPMAA 1933, LAASIMER 1965, ZOBEL 1987, PÄRTEL et al. 1999a), eine syntaxonomische Klassifikation mit umfassender Gesamtbetrachtung sämtlicher vorkommenden Trockenrasentypen sowie deren Einbindung in ein überregionales System mit formal gültigen Syntaxonbezeichnungen fehlt jedoch bislang. Besonders die älteren Vegetationsklassifikationen (z. B. LIPPMAA 1933, TOMSON 1937) sind nicht mit dem allgemein gültigen syntaxonomischen System vereinbar, da keine Holozönosen, sondern Synusien klassifiziert wurden. Außerdem sind die bis dato vorgenommenen informellen Einteilungen in vielen Fällen von den Autoren nicht durch Vegetationstabellen dokumentiert worden und somit schwer nachvollziehbar. Die vorliegenden Arbeiten beschränkten sich weitgehend auf die Untersuchung der Alvartrockenrasen. ZoBEL (1987) gibt dazu eine detaillierte Übersicht, indem er die klassifikatorische Einteilung verschiedener Autoren vergleicht. Zusätzlich nimmt er eine Einteilung anhand eigener Aufnahmen vor. PÄRTEL et al. (1999a) führten an einem umfangreichen Datensatz von Vegetationsaufnahmen sämtlicher Alvargebiete Estlands eine floristische Clusteranalyse durch und unterschieden dadurch sieben unterschiedliche Alvargesellschaften. Diese ordneten sie jedoch nicht einer formellen syntaxonomischen Hierarchie unter, sondern verwendeten die 
von AlBERTSON (1950) erstellte informelle Nomenklatur. Die außerhalb der Alvare vorkommenden Trockenrasentypen, insbesondere die Sandtrockenrasen, blieben jedoch bis auf wenige Erwähnungen (LIPPMAA 1933, TOMSON 1937, LAASIMER 1965) unerforscht. Sämtliche Studien beschränkten sich zudem auf die Bearbeitung von Gefäßpflanzen, während Moose nur selten und Flechten nie berücksichtigt wurden.

Im Rahmen dieser Diplomarbeit soll daher die syntaxonomische Klassifikation der unterschiedlichen Trockenrasentypen auf Saaremaa durch territorial gültige Charakter- und Differenzialarten unter gleichrangiger Beachtung von Gefäßpflanzen und Kryptogamen (in dieser Arbeit Moose, Flechten, „Makroalgen“) neu herausgebildet sowie die synsystematische Stellung der Trockenrasen auf Saaremaa im überregionalen Kontext diskutiert werden.

Im Mittelpunkt stehen dabei folgende Fragen:

- Welche Trockenrasengesellschaften können auf Saaremaa unterschieden werden?

- Wie unterscheiden sich die vorkommenden Trockenrasentypen voneinander?

- Lassen sich die unterschiedenen Gesellschaften in ein überregionales System mit formal gültigen Syntaxonbezeichnungen einfügen?

Es wird angestrebt, anhand dieser syntaxonomischen Klassifikation sämtliche auf Saaremaa vorkommenden Trockenrasentypen floristisch und standörtlich zu charakterisieren. Die auf diese Weise differenzierten Einheiten sollen zudem als Grundlage für eine Untersuchung der Phytodiversitätsmuster dienen.

Die Erforschung der Phytodiversitätsmuster von Offenlandgesellschaften sowie deren Ursachen und Gradienten waren in jüngerer Vergangenheit das Forschungsziel zahlreicher Wissenschaftler. In der Literatur werden Zusammenhänge der Phytodiversität mit abiotisch großräumigen (z. B. BEGON et al. 1998: 601 ff.), abiotisch lokalen (z. B. PÄRTEL \& ZOBEL 1999, PÄRTEL et al. 1999a, EWALD 2003, LUNDHOLM \& LARSON 2003) sowie biotischen Parametern (z. B. CONNELL 1978, ZOBEL 1992) angeführt.

Im Rahmen dieser Diplomarbeit sollen die Phytodiversitätsmuster der Trockenrasen auf Saaremaa unter Einbeziehung der bereits vorliegenden Theorien und Hypothesen untersucht werden. Primär sollen dabei folgende Fragen geklärt werden:

- Unterscheiden sich die auf Saaremaa vorkommenden Trockenrasentypen hinsichtlich ihrer mittleren Artenzahlen auf einer Fläche von $4 \mathrm{~m}^{2}$ ?

- Welche Anteile am Artenreichtum auf einer $4 \mathrm{~m}^{2}$ großen Fläche haben dabei die Artengruppen (Gefäßpflanzen, Moose und Flechten) in den unterschiedlichen Trockenrasentypen?

- Welchen Einfluss haben abiotische Parameter auf den Artenreichtum, und wird der Artenreichtum der Artengruppen in unterschiedlicher Weise beeinflusst?

- Welchen Einfluss haben biotische Parameter auf den Gesamtartenreichtum, und lassen sich Indizien für eine interspezifische Konkurrenz ableiten? 


\section{Das Untersuchungsgebiet}

\subsection{Lage und allgemeine Gebietsbeschreibung}

Die estnische Ostseeinsel Saaremaa liegt etwa $20 \mathrm{~km}$ westlich der Festlandsküste zwischen $57^{\circ} 50^{\circ}$ und $58^{\circ} 40^{\circ}$ nördlicher Breite und zwischen $21^{\circ} 45^{\circ}$ und $23^{\circ} 20^{\circ}$ östlicher Länge. Sie ist mit einer Fläche von $2.673 \mathrm{~km}^{2}$ die größte estnische Insel. Das gesamte Archipel bildet den Landkreis Saaremaa und hat eine Fläche von $2.922 \mathrm{~km}^{2}$ (MARDISTE 2002: $13 \mathrm{f}$.). Ihre Ausdehnung von Osten nach Westen beträgt etwa $55 \mathrm{~km}$ und von der im Süden liegenden Hauptstadt Kuressaare bis zum nördlichsten Punkt Pammana etwa $30 \mathrm{~km}$. Vom südlichsten Punkt Sääre auf der Halbinsel Sörve bis zum nördlichsten Punkt beträgt die Entfernung ca. $55 \mathrm{~km}$.

Im Jahre 2000 lebten 36.022 Menschen auf Saaremaa, 14.932 davon in der Hauptstadt Kuressaare, 21.090 verteilt auf den übrigen Teil der Fläche (MARKSOO 2002: 416). Dies entspricht einer Bevölkerungsdichte von etwa 13,5 Einwohner $/ \mathrm{km}^{2}$, bzw. ca. 8,5 Einwohner/ $\mathrm{km}^{2}$ im Umland, rechnet man Kuressaare ab.

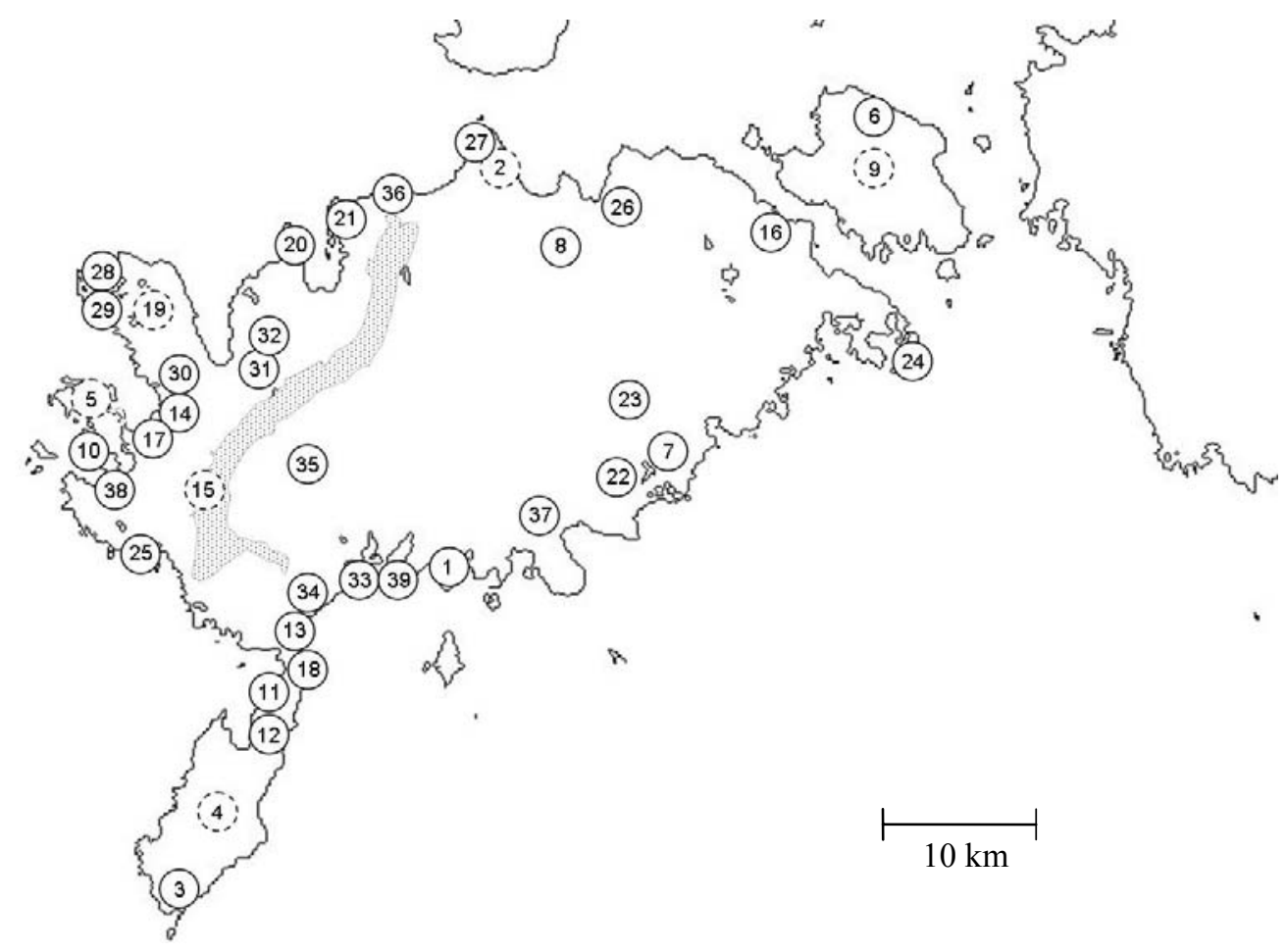

Karte 1: Die Insel Saaremaa mit den im Text erwähnten Orten (durchgehende Kreislinie) und Gebieten (gestrichelte Kreislinie) sowie dem angedeuteten Verlauf der Endmoräne (,West-Saaremaa-Hochland“). Kuressaare (1), Halbinsel Pammana (2), Sääre (3), Halbinsel Sörve (4), Insel Vilsandi (5), Raugi (Muhu) (6), Uue-Lõve (7), Karja (8), Muhu (9), Eeriksaare (10), Kaugatoma pank (11), Lõu pank (12), Salme (13), Kihelkonna (14), Naturschutzgebiet Viidumäe (15), Orrissaare (16), Halbinsel Papissaare (17), Läätsa (18), Halbinsel Tagamoisa (19), Tagaranna pank (20), Panga pank (20), Poka (21), Valjala (22), Halbinsel Kübassaare (23), Katri pank (24), Pidula laht (25), Metsa (26), Murika (27), Uudepanga laht (28), Haagi lõugas (29), Kehila (30), Kõõru (31), Pidula laht (32), Mändjala (33), Tehumardi (34), Kärla (35), Varese Sadam (36), IIpla (37), Atla (38), Nasva (39). 


\subsection{Geologie und Geomorphologie}

Auf Saaremaa stehen silurische Kalkgesteine an (ausführlich in RAUKAS \& TEEDUMÄE 1997: 35 ff.), die größtenteils von quartären Ablagerungen bedeckt sind. Besonders die letzte Vereisung des Pleistozäns prägt das Landschaftsbild der Insel. Die in Karte 1 angedeutete Endmoräne bildet mit einer Höhe von 34-54 m ü. NN das „West-Saaremaa-Hochland“, dessen höchstgelegene Bereiche vor etwa 10.300 Jahren erstmals aus dem Baltischen Eismeer auftauchten. Während der folgenden Entwicklungsstadien der Ostsee, dem Yoldiameer, dem Ancylussee, dem Litorinameer und dem Limneameer kamen zunächst vor ungefähr 7.500 Jahren die 22-35 m ü. NN hohen Ausläufer des Hochlandes und danach sukzessive die restlichen Gebiete Saaremaas zum Vorschein (POSKA \& SAARSE 2002: 556). Auf den tiefer gelegenen Bereichen der Insel lagerten sich während der Überflutungszeiten marine Sedimente ab (1. c.: 125 ff.), welche durch das Meer und, nachdem sich das Land aus dem Wasser emporgehoben hatte, teilweise durch Erosionsprozesse abgetragen oder verlagert wurden. In einigen Bereichen wurde das Grundgestein durch geologische Prozesse und anthropogene Einflüsse fast vollständig freigelegt, wodurch Alvare entstehen konnten (1. c.: 252 ff.). An anderen Stellen im westlichen Teil der Insel und in Küstennähe bildeten sich Dünen, die hauptsächlich aus Feinsand bestehen (1. c.: 292).

\subsection{Böden}

Bedingt durch die in Abschnitt 2.2 beschriebenen geologischen Prozesse sind Sand oder Lehm die vorherrschenden Bodenarten auf Saaremaa. (TERAS 2002: 131). Eine entscheidende Rolle bei der Pedogenese spielte der zumeist hohe Carbonatgehalt des Ausgangsmaterials. Unmittelbar über Kalkgesteinen bildeten sich flachgründige Rendzinen. Aus solchen Rendzinen und über Geschiebemergel kam es bei schwankenden Wasserverhältnissen zur Entstehung von (hydromorphen) Gley-Rendzinen oder Gley-Braunerden. In Sümpfen und Niedermooren entstanden durch die ständige Überstauung des Wassers Moorböden. An einigen mit Pinus bewachsenen Stellen der Insel kommen Podsole vor, die sich aus basenarmen oder bereits stark entkalkten Sanden entwickelten (REINTAM 1995: 124 ff.).

\subsection{Klima}

Das Klima Saaremaas ist schwach ozeanisch bis schwach kontinental geprägt. Von Dezember bis März liegen die mittleren Temperaturen meist unter dem Gefrierpunkt und selbst im April bei nur 2,6-3 ${ }^{\circ} \mathrm{C}$ (Tab. 1). Die Vegetationsperiode (Definition: Tage ohne Nachtfrost) beginnt dementsprechend relativ spät und dauert auf Saaremaa etwa 150-180 Tage, auf Vilsandi 190 Tage pro Jahr. Februar ist der kälteste, Juli der wärmste Monat des Jahres (JõGI 2002: 68). Im Februar liegen die mittleren Bodentemperaturen zwischen -4 und $-5^{\circ} \mathrm{C}$, im Juli bei etwa $20{ }^{\circ} \mathrm{C}$ und im Jahresmittel bei $6{ }^{\circ} \mathrm{C}$ (ANONYMus 1970: $108 \mathrm{ff}$.).

Die mittleren jährlichen Niederschlagsmengen variieren je nach Messpunkt zwischen 540 und $675 \mathrm{~mm}$. Während der Sommermonate fallen von Mai bis August 192-246 mm, in der Hauptvegetationsperiode von April bis Oktober 349-429 mm und außerhalb der Vegetationsperiode von November bis März 191-246 mm Niederschlag (Tab. 2) (RAUDSEPP \& JAAGUS 2002: 67).

Es lässt sich ein leichtes Kontinentalitätsgefälle von Westen nach Osten feststellen: Die mittleren Temperaturen in den Wintermonaten sind im Westen etwa $2{ }^{\circ} \mathrm{C}$ höher als im Osten (JÕGI 2002: 68).

Jährlich werden im äußersten Westen der Insel durchschnittlich 70 Tage mit geschlossener Schneedecke verzeichnet. Wenige Kilometer weiter östlich sind es bereits 80 Tage, entlang des Endmoränenverlaufes sowie an der Südküste 90 Tage und im Zentrum bis in den nordöstlichen Teil sogar 100 Tage (ANONYMUS 1970: 185). 
Tab. 1: Mittlere Monats- und Jahrestemperaturen $\left[{ }^{\circ} \mathrm{C}\right]$ an unterschiedlichen Messpunkten (RAUDSEPP \& JAAGUS 2002: 66, vereinfacht).

\begin{tabular}{|l||cccccccccccc|c|}
\hline \multicolumn{1}{|c||}{ Messpunkte } & \multicolumn{110}{|c|}{ Monate } \\
\hline \hline & I & II & III & IV & V & VI & VII & VIII & IX & X & XI & XII & Mittel \\
Vilsandi & $-2,3$ & $-3,4$ & $-1,9$ & 3,0 & 8,0 & 12,6 & 16,3 & 16,1 & 12,6 & 7,6 & 3,4 & 0,1 & 6,0 \\
Sõrve & $-2,6$ & $-3,5$ & $-2,0$ & 2,7 & 8,1 & 12,9 & 16,5 & 16,0 & 12,6 & 7,7 & 3,4 & $-0,1$ & 6,0 \\
Kuressaare & $-3,6$ & $-4,6$ & $-3,3$ & 2,8 & 9,0 & 13,5 & 16,7 & 15,8 & 11,9 & 6,8 & 2,6 & $-0,8$ & 5,6 \\
Raugi (Muhu) & $-4,0$ & $-5,3$ & $-3,5$ & 2,6 & 9,1 & 14,0 & 16,9 & 15,9 & 11,9 & 6,6 & 2,3 & $-1,3$ & 5,4 \\
\hline
\end{tabular}

Tab. 2: Mittlere Monats- und Jahresniederschlagsmengen [mm], sowie die Niederschlagsmengen während der Sommermonate Mai bis August, der Hauptvegetationsperiode April bis Oktober und außerhalb der Vegetationsperiode November bis März an unterschiedlichen Messpunkten (RAUDSEPP \& JAAGUS 2002: 67).

\begin{tabular}{|l||cccccccccccc|ccc|c|}
\hline \multicolumn{1}{|c||}{ Messpunkte } & \multicolumn{110}{c|}{ Monate } \\
\hline \hline & I & II & III & IV & V & VI & VII & VIII & IX & X & XI & XII & V-VIII & IV-X & XI-III & Gesamt \\
Vilsandi & 38 & 28 & 27 & 29 & 34 & 36 & 50 & 72 & 62 & 66 & 54 & 44 & 192 & 349 & 191 & 540 \\
Sõrve & 47 & 34 & 31 & 30 & 38 & 40 & 52 & 76 & 63 & 60 & 61 & 50 & 206 & 359 & 223 & 582 \\
Kuressaare & 42 & 30 & 30 & 37 & 37 & 41 & 64 & 74 & 71 & 59 & 61 & 48 & 216 & 383 & 211 & 594 \\
Uue-Lõve & 48 & 34 & 34 & 41 & 41 & 47 & 73 & 85 & 74 & 68 & 69 & 61 & 246 & 429 & 246 & 675 \\
Karja & 46 & 33 & 27 & 33 & 40 & 45 & 70 & 87 & 77 & 71 & 67 & 52 & 242 & 423 & 225 & 648 \\
Raugi (Muhu) & 31 & 19 & 21 & 29 & 38 & 45 & 58 & 78 & 66 & 54 & 57 & 36 & 219 & 368 & 164 & 532 \\
\hline
\end{tabular}

Die mittleren Windgeschwindigkeiten sind im Westen der Insel höher als im Osten (Karte 2). Im Zeitraum von 1992 bis 2001 wurde auf Vilsandi eine mittlere Windgeschwindigkeit von $6,86 \mathrm{~m} / \mathrm{s}$ und in Kuressaare von 5,66 m/s gemessen (RATHMANN 2003: 29).

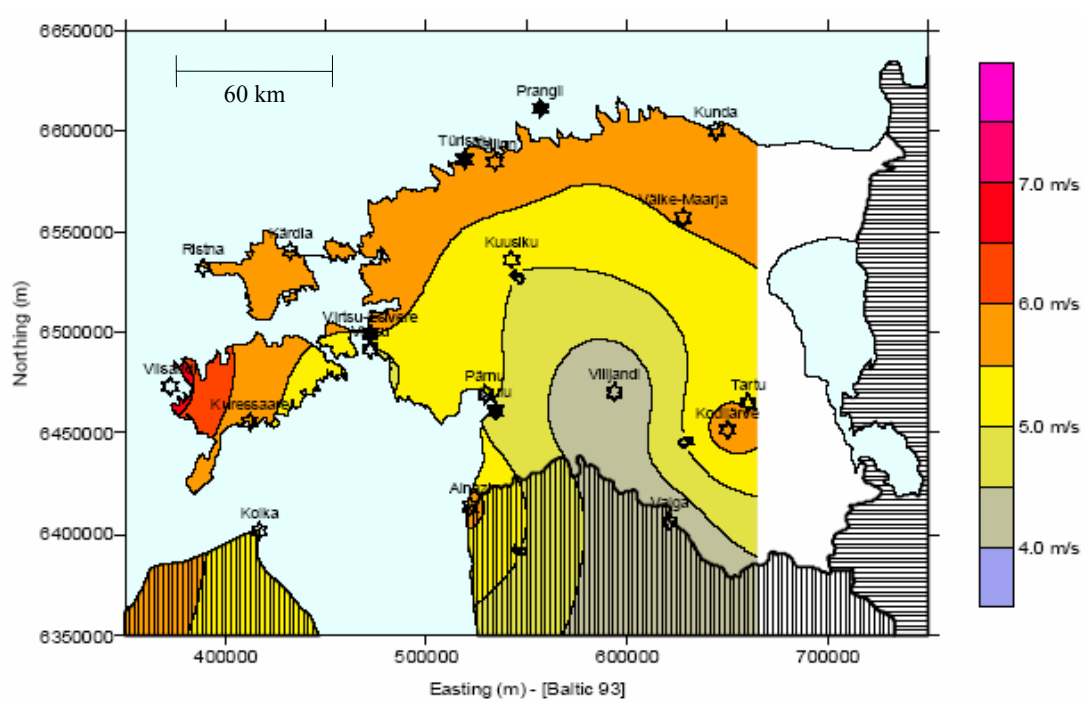

Karte 2: Mittlere Windgeschwindigkeiten Estlands (RATHMANN 2003: 33, leicht verändert). 


\subsection{Flora und Vegetation}

TRASS (2002: 126) teilt Saaremaa nach der vorherrschenden Vegetation in sechs „geobotanische Mikroregionen“ ein. Charakteristisch für Nord-Saaremaa sind artenreiche Laubwiesen. In Ost-Saaremaa und Muhu nehmen Alvare 12 bis 20 \% der Fläche ein. Der Rest dieser Region besteht aus einem Mosaik von Hainwäldern und Sümpfen. Im Bereich des mittleren Landrückens kommen artenreiche Niedermoore, Laubwiesen, Heide- und Preiselbeer-Kiefernwälder vor. Mittel-Saaremaa ist geprägt durch artenreiche Laubwiesen und Niedermoore sowie versumpfte oder feuchte Mähwiesen. Süd-Saaremaa ist durch artenreiche Laubwiesen, Hainwälder, Alvare und Küstenvegetation charakterisiert. Entlang der SüdKüste erstreckt sich ein etwa 3,5 km langer und bis zu $8 \mathrm{~m}$ hoher Dünenstreifen. In WestSaaremaa herrschen Alvare vor. Stellenweise findet man Zonen mit Küstenvegetation.

Geobotanisch betrachtet liegen die baltischen Staaten im Übergangsbereich von der borealen zur temperaten Zone in der hemiborealen Region (LANG et al. 2003: 97). Estland befindet sich somit an der Südgrenze der Taiga (LAASIMER 1969: 68). Von Nordost- nach SüdwestEstland (ungefähr von Kunda über Rakvere und Viljandi nach Mõisaküla), entlang der höchsten postglazialen Küstenlinie, verläuft eine floristische Grenze, die das Land in zwei phyto-geografische Untereinheiten teilt. Die Vegetation westlich dieser Grenze ist reicher an ozeanischen und an das kalkhaltige Grundgestein angepassten Arten (LIPPMAA 1935). Der Autor teilt Estland in unterschiedliche phytogeografische Distrikte ein. Saaremaa befindet sich im Distrikt „Estonia maritima insularis“, der in einen westlichen („Estonia maritima occidentalis“) und einen östlichen Teil (,Estonia maritima orientalis“) unterteilt wird.

\subsubsection{Florenausstattung}

Aufgrund der geografischen Lage Estlands treffen verschiedene Florenelemente mit unterschiedlichen Anteilen an Gefäßpflanzenarten zusammen: arktisches und arktisch-alpines (2\%), eurasisch-boreoamerikanisches $(24,7 \%)$, eurasiatisches $(25,4 \%)$, eurosibirisches $(6,2 \%)$, europäisches $(23,5 \%)$, montanes $(0,9 \%)$, atlantisches (pseudoatlantisches) $(1,3 \%)$, pontisches und ponto-sarmatisches $(2,8 \%)$, mediterranes $(0,5 \%)$, boreotropisches (kosmopolitisches) Element (6,3\%) und Arten mit unbestimmter Arealzuordnung (6,5\%) (LIPPMAA 1935).

In Estland stoßen 455 Gefäßpflanzenarten (30 \%) an ihre Verbreitungsgrenze (KUKK 1999 zit. n. KULL et al. 2002: 174). Auf den westlichen Inseln überlappen sich vorwiegend die Verbreitungsgrenzen einer Reihe subatlantischer Arten und Steppenarten (EILART 1969: 22).

Auf Saaremaa gibt es schätzungsweise 1150 Gefäßpflanzenarten. Das sind etwa $2 / 3$ der in Estland vorkommenden Arten (REITALU \& RoOSALUSTE 2002: 96). Im Jahr 2002 waren 224 Laubmoos-, 46 Lebermoos- (KANNUKENE 2002: 95) und 464 Flechtenarten (RANDLANE 2002: 111) (ca. $70 \%$ der in Estland registrierten Flechten) aus dem Untersuchungsgebiet bekannt.

\subsubsection{Natürliche Vegetation}

In der „Karte der natürlichen Vegetation Europas“ (BOHN \& NEUHÄUSL 2000, BOHN et al. 2000, 2003, 2004) sind die im Folgenden beschriebenen natürlich vorkommenden Vegetationseinheiten für Saaremaa aufgeführt. Die Abgrenzungen der jeweiligen Kartiereinheiten verdeutlicht Karte 3.

In den im Norden und Westen Estlands vorkommenden Alvar-Fichtenmischwäldern (D18) der hemiborealen Region herrschen Nadelbaumarten wie Picea abies oder Pinus sylvestris vor. Quercus robur und Fraxinus excelsior sind stellenweise beigemischt. Sie sind meist licht und durch eine reiche Strauch- (Juniperus communis, Corylus avellana; Ribes alpinum, 
Lonicera xylosteum, Cotoneaster integerrimus, Sorbus aucuparia, Daphne mezereum, Rhamnus cathartica) und Krautschicht aus xerophytischen Heidewaldarten (Arctostaphylos uva-ursi, Antennaria dioica, Sedum acre, Thymus serpyllum) und Trockenrasen- bzw. Wiesensteppenpflanzen (Filipendula vulgaris, Asperula tinctoria, Brachypodium pinnatum, Sesleria caerulea, Carex flacca) gekennzeichnet. Da flachgründige Böden und Trockenheit im Sommer die limitierenden Faktoren für den Wald darstellen, stehen sie auf extremeren (Fels-)Standorten z. T. im Wechsel mit Trockenrasen, thermophilen Saumgesellschaften und xerophytischen Gebüschen (BOHN et al. 2000: 30, 2003: 186).

Die baltisch-nordwestsarmatischen Laub-Fichtenmischwälder (D19) zeichnen sich durch eine geschlossene Baumschicht von Picea abies aus. Die als zweite Baumschicht oder als Unterwuchs auftretenden Laubhölzer Quercus robur, Acer platanoides und Fraxinus excelsior werden durch Winterfröste geschädigt und erreichen deshalb nur selten die obere Baumschicht. Die Strauchschicht wird von Arten wie Corylus avellana, Ribes alpinum und Cornus sanguinea gebildet. Die Krautschicht mit Arten wie Vaccinium myrtillus, Oxalis acetosella, Maianthemum bifolium, Hepatica nobilis, Stellaria holostea und Ranunculus cassubicus ist meist gut entwickelt. Moosarten wie Dicranum scoparium, D. polysetum, Rhytidiadelphus triquetrus sind vertreten (BoHN et al. 2000: 30, 2003: 186 f.).

In den osteuropäischen psammophytischen Kiefernwäldern (D49) kommen neben Zwergsträuchern (Vaccinium vitis-idaea, V. myrtillus, Calluna vulgaris) xerophythische Arten wie Festuca polesica, Carex ericetorum, Koeleria glauca und Dianthus arenarius, Pseudolysimachion spicatum, sowie Flechten und Moose vor. Sie besiedeln vorwiegend trockene und basenarme Sandstandorte (BOHN et al. 2003: 202).

Bei den Linden-Eichen-Mischwäldern (F70) des Baltikums handelt es sich um isolierte westliche Vorposten ihres weiter östlich gelegenen Hauptverbreitungsgebietes. Quercus robur, Tilia cordata und z. T. Picea abies sind die vorherrschenden Baumarten. Ulmus glabra, Acer platanoides und Populus tremula sind häufig beigemischt (BOHN et al. 2003: $281 \mathrm{f}$.).

Die nordostbaltische Salzvegetation (P21) mit Glaux maritima, Juncus gerardii und Salicornia europaea ist durch das Vorkommen von Atriplex calotheca charakterisiert (BOHN et al. 2003: 441).

Baltische Sphagnum magellanicum-Hochmoorkomplexe (S9) sind durch Arten wie Calluna vulgaris, Empetrum nigrum und Trichophorum cespitosum ssp. cespitosum gekennzeichnet (BOHN et al. 2000: 73).

Kalkreiche Braunmoore (S24) sind von Braunmoosen und Seggen oder anderen Cyperaceen dominierte Niedermoore (BOHN et al. 2000: 74, 2003: 468). 


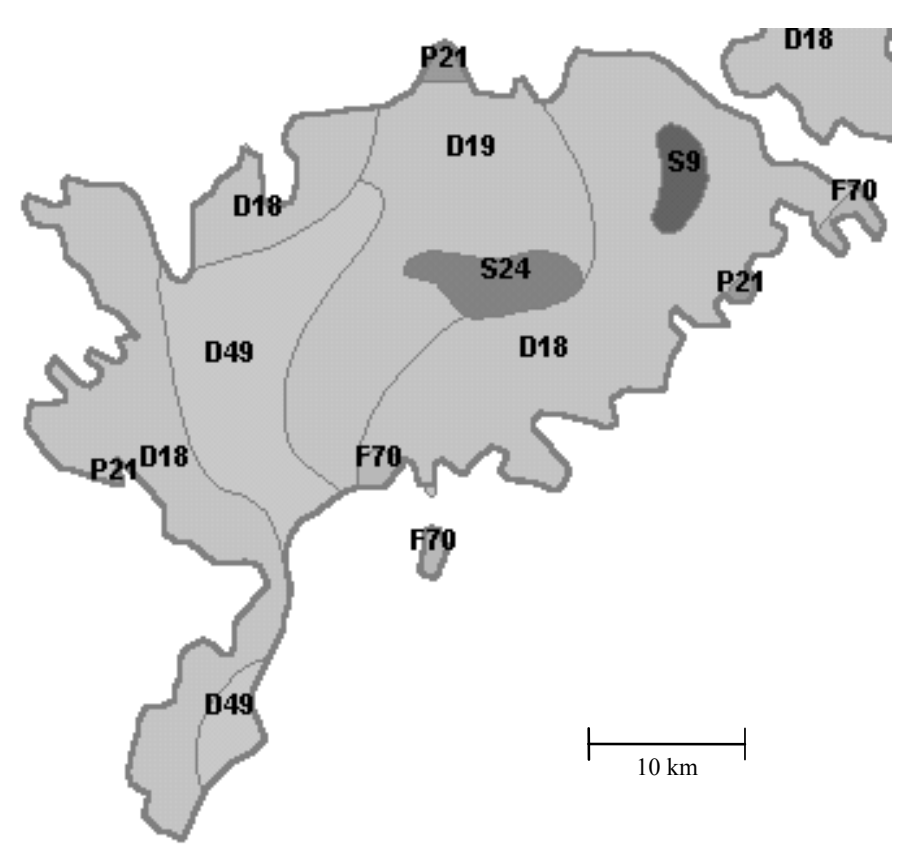

Karte 3: Die natürliche Vegetation Saaremaas (aus BoHN et al. 2004); D18= Alvar-Fichtenmischwälder, D19 = Baltisch-nordwestsarmatische Laub-Fichtenmischwälder, D49= Osteuropäische psammophytische Kiefernwälder, F70 = Linden-Eichen-Mischwälder, P21 = Nordostbaltische Salvegetation, S9=Baltische Sphagnum magellanicum-Hochmoorkomplexe, S24 = Kalkreiche Braunmoore.

\subsubsection{Vegetationsgeschichte und anthropogene Einflüsse}

Die Vegetationsgeschichte und anthropogenen Einflüsse wurden durch Pollen- und Sporenanalysen von See- und Sumpfsedimenten aus unterschiedlichen Gebieten Saaremaas zurückverfolgt (z. B. SAARSE \& KÖNIGSSON 1992, VESKI 1998, POSKA \& SAARSE 2002).

\section{Vegetationsgeschichte}

Kurze Zeit nachdem Teile des „West-Saaremaa-Hochlands“ aus dem Baltischen Eismeer auftauchten (vgl. Abschnitt 2.2), fanden sich erste Pionier- (z. B. Betula, Pinus, Corylus, Ulmus, Hippophae, Populus, Salix, Juniperus) und Ruderalarten (Artemisia, Chenopodiaceae, Rumex acetosa/ acetosella, Polygonum arviculare) ein. Natürliche Offenlandschaften und lichte Wälder bestimmten das Erscheinungsbild der Insel. Im Boreal begannen erste thermophile Laubbaumarten (Alnus, Quercus, Tilia) Fuß zu fassen. Sie breiteten sich aus und bildeten während des Atlantikums geschlossene Wälder, was einen merklichen Rückgang der Krautschicht zur Folge hatte. Anthropogene Störungen zeigten sich durch Veränderungen in der Zusammensetzung des Waldes und das verstärkte Auftreten von Apophythen und Ruderalarten. Vor etwa 3.500 Jahren hatten sich überall Mischwälder mit einem hohen Anteil von Picea etabliert. Die vergangenen tausend Jahre sind durch Zeigerarten für offene Landschaften, Felder und Wiesen gekennzeichnet. Laubbäume spielen eine zu vernachlässigende Rolle (POSKA \& SAARSE 2002: 558).

\section{Anthropogene Einflüsse}

Erste permanente menschliche Besiedlungen auf Saaremaa wurden anhand von Steingräbern bei archäologischen Untersuchungen in Alvar-Gebieten bei Võhma nachgewiesen und auf das Mesolithikum (5.000-4.000 v. Chr.) zurückdatiert (LõUGAS 1988: 348 ff.). Früheste anthropogene Beeinflussungen der Vegetation wurden in der Nähe dieser Ausgrabungsstätten vor etwa 6.500-6.000 Jahren verzeichnet. Besonders die ausgedehnten Laubwälder wurden zu 
dieser Zeit genutzt und einzelne Flächen gerodet, wodurch ein Anstieg des Artenreichtums sowie der Häufigkeit von krautigen Arten zu verzeichnen war. Vor 4.500-4.200 Jahren wurden erste Getreide-Felder (Triticum-, Avena- und Hordeum-Typ) angelegt und die Landschaft weiter aufgelichtet. Laubwälder wichen Feldern und Weiden und die Anzahl der Arten stieg auf das bisherige Maximum an (PoSKA \& SAARSE 2002: $559 \mathrm{ff}$.). Durch das Entfernen der Sträucher und den Kahlschlag der Alvarwälder mit anschließender Heumahd oder Beweidung konnten sich im Laufe der Zeit die charakteristischen artenreichen Alvargesellschaften ausbilden (LAASIMER 1975: 102 f.). Nach dem zweiten Weltkrieg gingen diese extensiven Nutzungsformen stetig zurück und die offenen Kulturlandschaften verbuschten größtenteils (VESKI 1996: 65). 


\section{Methoden}

\subsection{Aufnahmeflächen und Erhebungen im Gelände}

\subsubsection{Verteilung und Auswahl der Aufnahmeflächen}

Mit Hilfe eines topografischen Kartensatzes der Insel Saaremaa (Eesti Baaskaart 1:50.000) war es möglich, unbewaldete Gebiete - also Gebiete, die potenziell als Trockenrasenstandorte in Frage kommen - zu lokalisieren und aufzusuchen.

Wie in Karte 4 erkennbar, habe ich die Aufnahmeflächen über das gesamte Untersuchungsgebiet verteilt, um sämtliche auf Saaremaa vorkommenden Trockenrasentypen zu erfassen. Besonders im westlichen Teil der Insel kommen viele gut ausgeprägte Trockenrasen vor, während der Osten hauptsächlich durch Kiefernwälder, Äcker, Fett- und Feuchtwiesen oder durch von Juniperus communis überwachsene ehemalige Trockenrasenflächen charakterisiert ist. Dies erklärt die Häufung der Aufnahmeflächen im westlichen Teil der Insel.

Die Auswahl der Aufnahmeflächen erfolgte nach dem von einigen Autoren geforderten Homogenitätskriterium (DIERßEN 1990: 15 ff., DIERSCHKE 1994: 150 f.; vgl. die Diskussion in DENGLER 2003: 121 ff.). Im Gelände habe ich visuell einheitlich erscheinende Vegetationsbestände ausgewählt. Durch eine möglichst große Anzahl von Vegetationsaufnahmen war zu erwarten, dass jeder Trockenrasentyp erfasst wird.

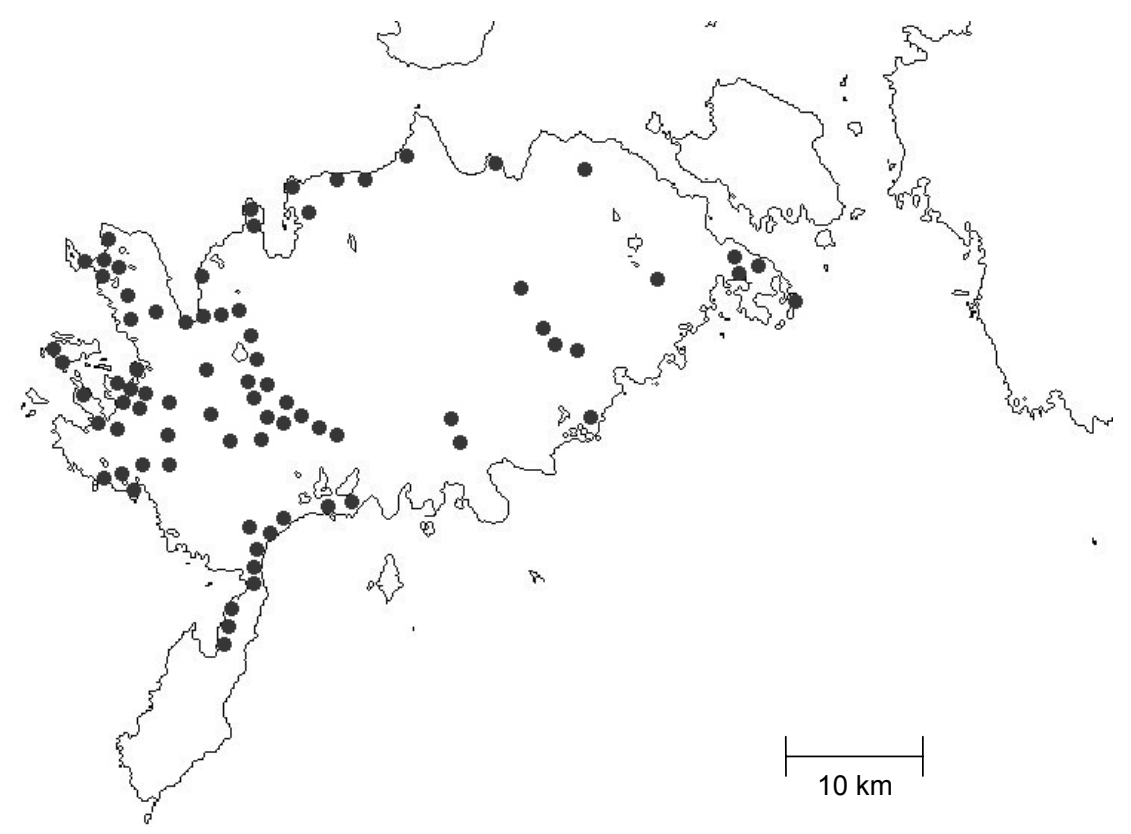

Karte 4: Verteilung der untersuchten Trockenrasenflächen über das Untersuchungsgebiet. 


\subsubsection{Vegetationsaufnahmen}

Im Zeitraum vom 29.05. bis 14.08.2004 habe ich 231 Vegetationsaufnahmen angefertigt. Zwei zeitversetzte Aufnahmen jeder Fläche zu erstellen, wie DiERSCHKE (1994: 149) es für lückige Trockenrasen und andere Vegetationstypen Mitteleuropas vorschlägt, war aus zeitlichen Gründen nicht möglich. Es ist daher anzunehmen, dass einige sommerannuelle Arten (z. B. Euphrasia spp.) in den zu Beginn der Vegetationsperiode angefertigten Aufnahmen unterrepräsentiert sind. Die frühjahrsannuellen Arten hingegen waren aufgrund des regenreichen Sommers noch bis zum Ende der Vegetationsperiode erkennbar.

Für die Vegetationsaufnahmen habe ich durchgängig $4 \mathrm{~m}^{2}$ große quadratische Flächen verwendet, um den Vergleich untereinander und mit Daten aus anderen Regionen, insbesondere jenen von LÖBEL (2002) aus Öland, zu ermöglichen. Dabei wurden sämtliche Gefäßpflanzen getrennt nach Schichten (Kraut- und Strauchschicht (letztgenannte $>0,5 \mathrm{~m}$ Wuchshöhe)) sowie Moose und Flechten einschließlich epigäischer, epilithischer, epiphytischer und epixyler Sippen erfasst. Ich habe alle in die Aufnahmefläche hineinragenden Pflanzenteile berücksichtigt. Im Gelände nicht oder schwierig anzusprechende Sippen, wie Arten aus den Gattungen Festuca und Hieracium, habe ich gesammelt und in den meisten Fällen eine Mischprobe der Moose und Flechten an unterschiedlichen Stellen der Aufnahmefläche genommen.

Die Vegetationsaufnahmen erfolgten nach der von DENGLER (2003: 131) vorgeschlagenen reinen Dominanzschätzskala (Tab. 3). Diese baut auf der kombinierten Abundanz-DominanzSchätzskala von BRAUN-BLANQUET (1951) in der modifizierten Fassung von WILMANNS (1993) auf. Unterhalb der $5 \%$ Deckung hat DENGLER (2003: 131) sie jedoch nach Vorschlag von PFADENHAUER et al. (1986: 60) feiner differenziert.

Tab. 3: Dominanzschätzskala nach DENGLER (2003: 131).

$\begin{array}{cl}\text { Symbol } & \text { Deckung [\%] } \\ \mathrm{r} & <1 \\ + & 1-2,5 \\ 1 & 2,5-5 \\ \mathrm{~A} & 5-10 \\ \mathrm{~B} & 10-25 \\ 3 & 25-50 \\ 4 & 50-75 \\ 5 & >75\end{array}$

Im Gelände habe ich zusätzlich die folgenden Parameter erfasst und die aufgeführten Abkürzungen im Tabellenkopf verwendet:

- Koordinaten (mit GPS eingemessen)

- Hangneigung [\%] 
- Exposition (Tab. 4)

Tab. 4: Abkürzungen der Exposition.

$\begin{array}{ll}\text { N } & \text { Nord } \\ \text { NE } & \text { Nordost } \\ \text { E } & \text { Ost } \\ \text { SE } & \text { Südost } \\ \text { S } & \text { Süd } \\ \text { SW } & \text { Südwest } \\ \text { W } & \text { West } \\ \text { NW } & \text { Nordwest }\end{array}$

- Den Gesteinsanteil des Oberbodens habe ich geschätzt (Tab. 5). Das geometrische Mittel habe ich aufgrund der besseren statistischen Auswertbarkeit der Daten gebildet. Für Gesteinsanteile unter $1 \%$ habe ich dabei den Wert 0,50 angenommen.

Tab. 5: Schätzskala für den Gesteinsanteil des Oberbodens.

$\begin{array}{llll}\text { Vol. [\%] } & \text { Bezeichnung } & \text { Symbol } & \text { geometrisches Mittel } \\ <1 & \text { Sehr schwach steinig } & 1 & 0,50 \\ 1-10 & \text { Schwach steinig } & 2 & 3,16 \\ 10-30 & \text { Mittel steinig } & 3 & 17,32 \\ 30-50 & \text { Stark steinig } & 4 & 38,73 \\ 50-75 & \text { Sehr stark steinig } & 5 & 61,24 \\ >75 & \text { Skelettboden } & 6 & 86,60\end{array}$

- Bodentiefgründigkeit [cm]: An fünf zufällig ausgewählten Stellen der Aufnahmefläche habe ich mithilfe eines $40 \mathrm{~cm}$ langen Eisenstabs die Tiefgründigkeit des Bodens ermittelt. Die jeweiligen Werte habe ich in eine Ordinalskala übertragen (Tab. 6) und danach den Median gebildet. Das arithmetische Mittel habe ich aufgrund der besseren statistischen Auswertbarkeit der Daten gebildet. Für über $40 \mathrm{~cm}$ tiefgründige Böden bin ich einen Kompromiss eingegangen und habe den Wert 50,0 angenommen.

Tab. 6: Skala der Kennwerte für die ermittelte Bodentiefgründigkeit.

$\begin{array}{lll}\text { Tiefgründigkeit }[\mathbf{c m}] & \text { Kennwert } & \text { arithmetisches Mittel } \\ 0-5 & 1 & 2,5 \\ 5-10 & 2 & 7,5 \\ 10-25 & 3 & 12,5 \\ 25-40 & 4 & 32,5 \\ >40 & 5 & 50,0\end{array}$


- Überstauung: In den Alvargebieten der Insel kommt es im Winter aufgrund der schlechten Drainageeigenschaften der harten Kalksteinplateaus durch Schmelzwasser auf gefrorenem Boden bzw. nach starken Regenereignissen auch in frostfreien Zeiten zu Überstauungen. Dies ließ sich anhand der etwas dunkleren Farbe des Bodens sowie der Steine, der teilweise verschlämmten Stellen, der Vegetation oder des ausgeprägten Mikroreliefs mit tieferen Senken vermuten oder nach starken Regenereignissen zuweilen erkennen. War dies der Fall vermerkte ich es mit +, anderenfalls mit -.

- Höhe ü. NN: Die Höhe ü. NN habe ich mithilfe der topographischen Karte bestimmt oder visuell in Relation zum Meeresspiegel geschätzt.

- Mikrorelief [cm]: Mittels Gliedermaßstab habe ich die Hoch- und Tiefpunkte der Bodenoberfläche und anschließend die Höhendifferenz ermittelt.

- Nutzungsform (falls erkennbar) und -intensität ( $-=$ Keine Nutzung, $\mathrm{O}=$ Gering, $+=$ Stark, $++=$ Sehr stark) (s.Tab. 7)

Tab. 7: Abkürzungen der Nutzungsformen.

$\begin{array}{ll}\text { KN } & \text { keine Nutzung } \\ \text { F } & \text { Befahren } \\ \text { T } & \text { Betreten } \\ \text { M } & \text { Mahd (zur Pflege oder Nutzung) } \\ \text { St } & \text { Straßenrand mit Pflegemahd } \\ \text { BP } & \text { Beweidung Pferde } \\ \text { BR } & \text { Beweidung Rinder } \\ \text { AB } & \text { Ackerbrache } \\ \text { IB } & \text { Industriebrache }\end{array}$

- Deckungsangaben [\%]: Vegetation gesamt und schichtenweise (Strauch-, Feld- und Boden- (Kryptogamen gesamt), Moos- und Flechtenschicht), Gesteine ( $>2 \mathrm{~mm})$, Bedeckungsgrad der Gesteine durch epilithische Flechten und Moose getrennt, Streu (totes organisches Material), Totholz; Betrug die Deckung unter $1 \%$ oder über $99 \%$ habe ich diese zur besseren Operationalisierbarkeit bei der statistischen Auswertung dem Wert $1 \%$ bzw. $99 \%$ zugeordnet.

- Strukturstraten: Von Kryptogamen besiedelte Substrate wie Totholz, lebende Pflanzenteile oder Gesteine habe ich getrennt aufgenommen und den Bedeckungsgrad der darauf vorkommenden Arten bezogen auf die Gesamtfläche geschätzt.

- Maximale und durchschnittliche Wuchshöhe der Vegetation gesamt und schichtenweise [cm]: Die maximale Wuchshöhe habe ich mittels Gliedermaßstab vermessen. Betrug der ermittelte Wert weniger als $1 \mathrm{~cm}$ habe ich $0,5 \mathrm{~cm}$ angegeben. Die durchschnittliche Wuchshöhe ist der ungefähre Mittelwert der Höhe aller in der Aufnahme befindlichen Pflanzen.

- Größe der Trockenrasengebiete: Mit einem Präzisions Digital-Planimeter („ottplan“, A. Ott Kempten) habe ich die im Gelände auf der topografischen Karte abgegrenzten Trockenrasengebiete vermessen und die dadurch ermittelten Werte in eine Ordinalskala übertragen (Tab. 8). Das arithmetische Mittel habe ich aufgrund der besseren statistischen Auswertbarkeit der Daten gebildet. Für Trockenrasengebiete, welche größer als 
$100.000 \mathrm{~m}^{2}$ waren, bin ich einen Kompromiss eingegangen und habe einen Wert von 200.000 angenommen.

Tab. 8: Skala der Kennwerte für die ermittelte Größe der Trockenrasengebiete

$\begin{array}{lll}\text { Größe des Trockenrasengebietes }\left[\mathbf{m}^{2}\right] & \text { Kennwert } & \text { arithmetisches Mittel } \\ \leq 100 & 1 & 50 \\ \leq 1.000 & 2 & 550 \\ \leq 10.000 & 3 & 5.500 \\ \leq 100.000 & 4 & 55.000 \\ >100.000 & 5 & 200.000\end{array}$

- Teilgebiete: Die Benennung der Aufnahmeflächen erfolgte nach einer Gliederung der Insel in Teilgebiete (Tab. 9, Karte 5). Die dort erstellten Vegetationsaufnahmen habe ich mit einer laufenden Nummer versehen.

Tab. 9: Abkürzungen der Teilgebiete.

E Eeriksaare (Halbinsel im Nationalpark Vilsandi)

J Sanddünen von Mändjala über Järve bis einschließlich Tehumardi

K Kaugatoma pank und Lõu pank (Küste und Alvar im Westen der Halbinsel Salme)

L Gebiet um Kihelkonna herum einschließlich des Naturschutzgebietes Viidumäe

N Nasva und Alvar westlich von Kuressaare

Ö Orrissaare, Pammana ps

P Papissaare ps (Halbinsel im NationalparkVilsandi)

S Norden der Halbinsel Salme (Orte Salme und Läätsa)

T Tagamoisa ps

V Vilsandi (Namensgebende Insel des Nationalparks)

X Gebiet um Kärla herum; im Norden bis Pidula laht, im Süden bis Katri pank

Y Ninase ps (Tagaranna pank) und Panga pank, östlich bis Poka

Z Gebiet um Valjala herum, östlich bis einschließlich Kübassaare ps 


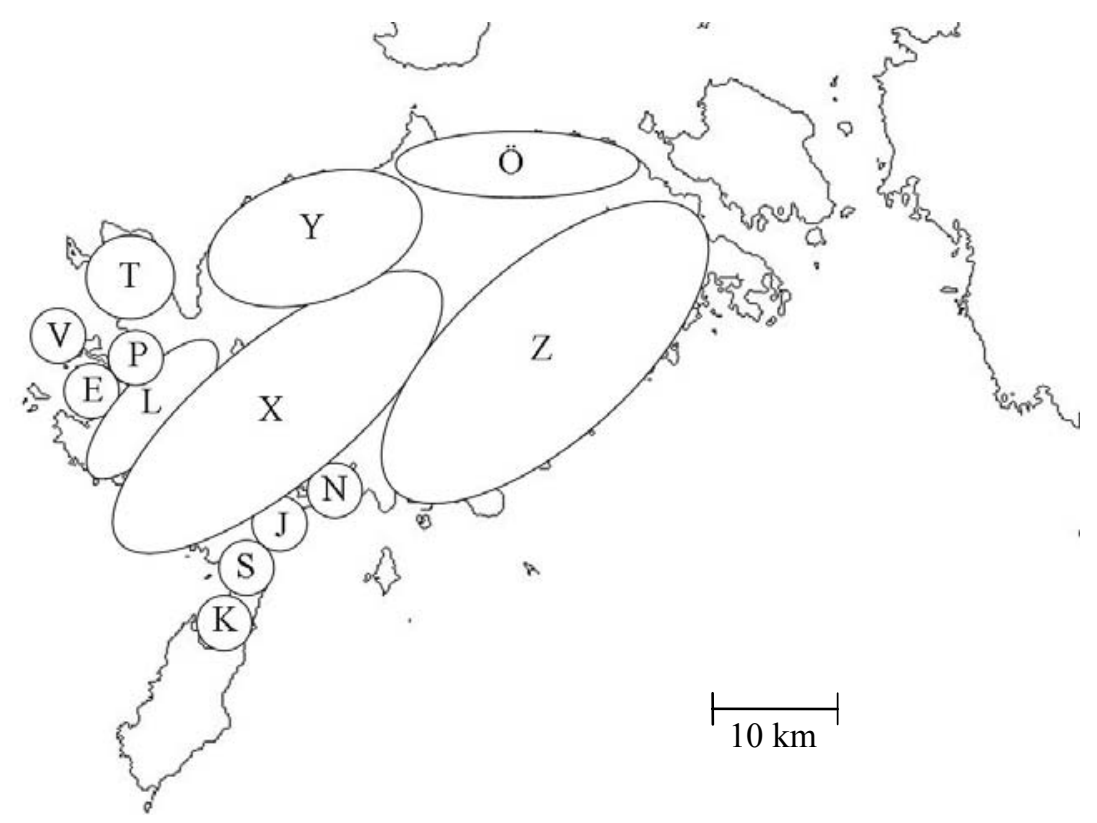

Karte 5: Gliederung der Insel in Teilgebiete. Die Vegetationsaufnahmen sind mit dem Kürzel des jeweiligen Teilgebietes versehen in welchem sie erstellt wurden.

\subsection{Sippennomenklatur und Bestimmungshilfen}

\subsubsection{Gefäßpflanzen}

Die Sippennomenklatur richtet sich nach WIßKIRCHEN \& HAEUPLER (1998). Darüber hinaus habe ich die folgenden Sippen unterschieden:

- Allium schoenoprasum L.: A. schoenoprasum ssp. schoenoprasum var. schoenoprasum und A. schoenoprasum ssp. schoenoprasum var. alvarense Hyl. (EICHWALD et al. 1984).

- Arenaria serpyllifolia (L.): A. sepyllifolia ssp. glutinosa (Mert. et W. J. D. Koch) Arcang. und A. sepyllifolia ssp. serpyllifolia (JÄGER \& WERNER 2001).

- Carex ornithopoda Willd.: Carex ornithopoda ssp. ornithopoda und C. ornithopoda ssp. elongata (Leyb.) Vierh. (JÄGER \& WERNER 2001).

- Crepis tectorum L.: C. tectorum („weed“-Form) und C. tectorum („outcrop“-Form) (sensu ANDERSSON 1990).

- Festuca oelandica (Hackel) K. Richter (TUTIN et al. 1980).

- Potentilla incana $\times$ Potentilla tabernaemontani (=Potentilla $\times$ subarenaria Borbás ex Zimmeter) (JÄGER \& WERNER 2001).

- Sesleria caerulea (L.) Ard. (TUTIN et al. 1980).

Die Bestimmung der Gefäßpflanzen erfolgte nach JÄGER \& WERNER (2001) und OBERDORFER (2001). In folgenden Fällen bin ich von der angegebenen Literatur abgewichen:

- Allium schoenoprasum ssp. schoenoprasum var. alvarense Hyl. habe ich nach den in EICHWALD et al. (1984) angegebenen Merkmalen bestimmt.

- Carex spp. habe ich in sterilem Zustand nach Neumann (1952), Doll (1988) bzw. EISELE \& ZÄHRINGER (1998) determiniert. 
- Crepis tectorum L.: Nach ANDERSSON (1990) habe ich die „outcrop“-Form von der „weed"-Form (ssp. tectorum) unterschieden.

- Festuca ovina agg. und F. rubra agg. habe ich nach DENGLER (1998) bestimmt.

- Jürgen Dengler (Lüneburg) hat Festuca oelandica (Hackel) K. Richter bestimmt und die Festuca ovina-Belege bestätigt. Das Einzelexemplar von Festuca oelandica hatte die gleichen morphologischen Merkmale wie die von Öland bekannte Art. Die zweifelsfreie Bestimmung ist jedoch bislang ausgeblieben, weshalb die Art mit „cf.“ aufgeführt wird.

- Die Bestimmung der Hieracium-Belege aus der Untergattung Pilosella (außer H. pilosella) hat Günther Gottschlich (Tübingen) übernommen.

Die folgenden Sippen habe ich zu Artengruppen zusammengefasst:

- Hieracium subgen. Pilosella und Hieracium subgen. Hieracium: War die Bestimmung bis auf Artebene aufgrund unzureichender Merkmalsausprägung der Pflanzen nicht möglich, habe ich diese nach JÄGER \& WERNER (2001) in die beiden Untergattungen getrennt.

- Potentilla argentea agg.: Den Potentilla argentea-Komplex (In JÄGER \& WERNER 2001 werden die unterschiedlichen Extremformen als Varietäten angegeben) habe ich nicht getrennt.

- Ranunculus auricomus agg. (=Ranunculus auricomus complex sensu MelzHEIMER in JÄGER \& WERNER 2001: 164 ff.)

- Rumex acetosa agg.: In sterilem Zustand habe ich die Arten R. acetosa und R. thyrsiflorus zusammengefasst, in fertilem Zustand jedoch unterschieden.

- Taraxacum sect. Erythrosperma (H. Lindb.) Dahlst.: Die Kleinarten habe ich nicht unterschieden.

- Taraxacum sect. Obliqua (Dahlst.) Dahlst.: Die Kleinarten habe ich nicht unterschieden.

- Taraxacum sect. Ruderalia Kirschner, H. Øllg. et Štěpánek: Die Kleinarten habe ich nicht unterschieden.

\subsubsection{Moose}

Die Nomenklatur der Laubmoose folgt CORLEY et al. (1981) mit Ergänzungen von CORLEY \& CRUNDWELl (1991), die der Lebermoose GROLLE \& LONG (2000). Im folgenden Fall wurde von den genannten Referenzwerken bei der Nomenklatur abgewichen:

- Hypnum cupressiforme Hedw.: Es wird FrAHM \& FREY $(1992,2004)$ folgend eine Unterscheidung der Varietäten cupressiforme und lacunosum Brid. vorgenommen.

Die Laubmoose habe ich nach Frahm \& Frey (1992, 2004), Nebel \& PhilliPI $(2000,2001)$, NyHOLM (1986, 1989, 1993, 1998), SMith (1978), die Lebermoose nach SMITH (1990), PATON (1999), DAMSHOLT (2002) und FRAHM \& FREY (2004) bestimmt.

Die folgende Sippe habe ich zu einer Artengruppe zusammengefasst:

- Schistidium apocarpum agg. (= Schistidium apocarpum complex sensu BLOM 1996). 


\subsubsection{Flechten}

Die Nomenklatur der Flechten richtet sich nach SANTESSON et al. (2004). Darüber hinaus habe ich die folgende Sippe unterschieden:

- Caloplaca lithophila H. Magn. habe ich nach Herk \& APTROOT (2004) von C. holocarpa unterschieden.

Die Determination erfolgte mithilfe des Mikroskopes, der Tüpfeltestmethode (K, C, P, J) und UV-Licht nach PURVIS et al. (1992), WIRTH (1995) und HERK \& APTROOT (2004). Im folgenden Fall bin ich von der angegebenen Literatur abgewichen:

- Agonimia globulifera Brand \& Diederich habe ich nach SÉRUSIAUX et al. (1999: 8 ff.) determiniert.

Die folgenden Sippen habe ich zu Artengruppen zusammengefasst:

- Cladonia pyxidata agg.: C. chlorophaea (Flörke ex Sommerf.) Spreng., C. cryptochlorophaea Asah., C. grayi G. Merr. ex. Sandst, C. merochlorophaea Asah., C. monomorpha Aptroot, Sipmann \& van Herk, C. novochlorophaea (Sipman) Brodo \& Ahti, C. pyxidata (L.) Hoffm.

\section{Dünnschichtchromatographie}

Einige Arten der Gattung Cladonia aus den Aufnahmen E04, E05, Ö04, T20, T21, T22, T23, T24, T25, T30, X14, X74, X39, X51, X52 und X54, sowie der Biodiversitätsplots 3, 4 und 12 bestimmte ich im Laufmittel A nach CULBERSON \& AMMANN (1979).

\subsection{4 „Makroalgen“"}

Die Determination der makroskopisch sichtbaren, freilebenden Cyano- und Phycobionten erfolgte nach WIRTH (1995: 56 ff.). Sie kommen potenziell als Assoziationspartner für eine Flechtensymbiose in Frage und wurden deshalb aufgenommen.

\subsection{Bodenökologische Untersuchungen}

\subsubsection{Probenahme}

Von sämtlichen Aufnahmeflächen habe ich eine Bodenmischprobe an drei zufällig ausgewählten Stellen aus dem durchwurzelten Teil des Oberbodens $(0-10 \mathrm{~cm})$ entnommen, bei ca. $105^{\circ} \mathrm{C}$ getrocknet und danach für den Transport luftdicht verschlossen.

\subsubsection{Bodenanalysen}

\section{Humusgehalt}

Der Humusgehalt der Böden wurde mittels Glühverlust im Muffelofen bestimmt. Hierzu habe ich jeweils $10 \mathrm{~g}$ der vorher über Nacht bei $105^{\circ} \mathrm{C}$ getrockneten und von größeren Wurzeln befreiten Probe bei $430^{\circ} \mathrm{C}$ bis zur Gewichtskonstanz verglüht (SCHLICHTING et al. 1995: 159). 


\section{Carbonatgehalt}

Den Carbonatgehalt habe ich nach Schlichting et al. (1995: 45 f.) durch das Beträufeln der Probe mit $10 \%$-iger Salzsäure und anschließender Beurteilung der optisch und akustisch erkennbaren Reaktion der $\mathrm{CO}_{2}$-Entwicklung geschätzt (Tab. 10). Bei Carbonatgehalten über $25 \%$ habe ich auf eine weitere Differenzierung aufgrund der zunehmenden Ungenauigkeit der Methode verzichtet. Das arithmetische Mittel habe ich aufgrund der besseren statistischen Auswertbarkeit der Daten gebildet. Bei Carbonatgehalten über 25,0\% bin ich einen Kompromiss eingegangen und habe einen Wert von 30,00 angenommen.

Tab. 10: Carbonatgehalt (leicht modifiziert nach ScHLICHTING et. al. 1995: 45 f.).

$\begin{array}{llll}\mathrm{CaCO}_{3}[\%] & \text { Bezeichnung } & \text { Kennwert } & \text { arithmetisches Mittel } \\ 0,0 \% & \text { Carbonatfrei } & 0 & 0,00 \\ <0,5 \% & \text { Sehr carbonatarm } & 1 & 0,25 \\ 0,5-2,0 \% & \text { Carbonatarm } & 2 & 1,25 \\ 2,0-10,0 \% & \text { Mäßig carbonathaltig } & 3 & 6,00 \\ 10,0-25,0 \% & \text { Carbonatreich } & 4 & 17,50 \\ >25,0 \% & \text { Sehr carbonatreich } & 5 & 30,00\end{array}$

\section{pH-Wert}

Die Messung des pH-Wertes erfolgte auf elektrometrischem Wege mittels einer GlasEinstabmesselektrode (WTW) in einer Suspension aus zermörsertem Boden und destilliertem Wasser im Verhältnis $1: 2,5$ (SCHLICHTING et al. 1995: $131 \mathrm{f}$.). Vor der Messung im klaren Überstand der Suspension habe ich die Proben etwa 20 Minuten lang maschinell geschüttelt und danach eine Stunde lang gewartet, bis sich der Boden abgesetzt hatte.

\section{Kationenaustauschkapazität und Basensättigung}

Die Kationenaustauschkapazität und Basensättigung habe ich nach der Methode von BROwN (1943) durchgeführt. Zur Extraktion der im Boden sauer reagierenden $\mathrm{H}^{+}$- und $\mathrm{Al}^{3+}$-Ionen wurden je 2,5 g Boden mit $25 \mathrm{ml}$ einer $1 \mathrm{M}$ Ammonium-Acetatlösung (H-Wert), zur Extraktion der austauschbaren Basen (z.B. $\mathrm{Ca}^{2+}, \mathrm{Mg}^{2+}, \mathrm{K}^{+}, \mathrm{Na}^{+}$) je 2,5 g Boden mit $25 \mathrm{ml}$ einer $1 \mathrm{M}$ Essigsäure (S-Wert) versetzt, unter gelegentlichem Schütteln zwei Stunden lang stehen gelassen und anschließend der $\mathrm{pH}-$ Wert der Suspension elektrometrisch gemessen. Zuvor habe ich Eichreihen erstellt, aus denen sich über die gemessenen $\mathrm{pH}$-Werte die Konzentrationen der basisch wirkenden Metallionen beziehungsweise sauer reagierenden $\mathrm{H}^{+}-$ und $\mathrm{Al}^{3+}$-Ionen in mval erschließen lassen. Hierzu wurden zu jeweils $100 \mathrm{ml}$ Natriumacetat bzw. Essigsäure sukzessive definierte Mengen von $0,1 \mathrm{M} \mathrm{HCl}$ bzw. $\mathrm{NaOH}$ mittels Bürette zugegeben. Nach jeder Zugabe erfolgte erneut die Messung des pH-Wertes. Die Konzentrationsänderung wurde nach folgender Gleichung berechnet und die ermittelten Werte anschließend auf Millimeterpapier gegen die gemessenen $\mathrm{pH}-$ Werte aufgetragen:

Konzentrationsänderung [mval/l] $=100 \mathrm{mval} / \mathrm{l} \times \mathrm{V}_{\text {zugegeben }} /\left(100 \mathrm{ml}+\mathrm{V}_{\text {zugegeben }}\right)$.

Die Summe von H- und S-Wert ergibt die Kationenaustauschkapazität (KAK) [mval/100 g]. Die Basensättigung [\%] wird aus dem Verhältnis des S-Wertes zur Kationenaustauschkapazität errechnet. 


\section{Bestimmung der Bodenart}

Die Bodenart habe ich durch Fingerprobe nach Schlichting \& al. (1995: 41) im Labor ermittelt und dabei zusätzlich ein $0,063 \mathrm{~mm}$-Sieb und eine Stereolupe zur genaueren Abschätzung des Schluffanteils zur Hilfe genommen. Die Benennung der Bodenarten erfolgte nach DIN 4220.

\subsection{Syntaxonomische Methoden}

\subsubsection{Klassifikatorisches Vorgehen}

Arbeitsgrundlage bildete die von DENGLER (2003) entwickelte, universell und überregional anwendbare Methode zur Vegetationsklassifikation. Wichtige Aspekte dieses Ansatzes sind die objektive Definition von Charakter- und Differenzialarten sowie die Möglichkeit, je übergeordnetem Syntaxon ein charakterartenfreies „Zentralsyntaxon“ der nächstunteren Hierarchieebene auszuweisen (1. c.:103 ff.).

Ich habe zunächst mittels Clusteranalyse (Sörensen-Index) in SORT 4.0 (ACKERMANN \& DURKA 1998) eine sehr grobe Einteilung der Vegetationsaufnahmen auf Klassenebene vorgenommen. Als Entscheidungsstütze bei der Vorsortierung wurden Vegetationstabellen aus Deutschland und Öland (DENGLER 2001b, 2001c, LöBEL 2002, DENGLER \& LÖBEL 2006) zur Hilfe genommen.

Das verwendete Konzept bezieht sich ausschließlich auf die prozentualen Stetigkeiten und richtet sich nach den folgenden Kriterien:

Das Differenzialartkriterium einer Art ist dann gegeben, wenn sie in einem Syntaxon mindestens die doppelte prozentuale Stetigkeit gegenüber einem anderen Syntaxon gleichen Ranges aufweist und dieser Stetigkeitsunterschied nicht zufallsbedingt ist (DENGLER 2003: 67). Eine Differenzialart unterhalb des Klassenniveaus erfüllt das „Differenzialartkriterium in einem oder mehreren Syntaxa gegenüber allen anderen gleichrangigen Syntaxa innerhalb des nächstübergeordneten Syntaxons“ und darf zudem in dem gegenübergestellten Syntaxon eine Stetigkeit von höchstens $20 \%$ besitzen (1. c.: 84). LöBEL (2002: 29) folgend werden mindestens zwei Artvorkommen in den Vegetationsaufnahmen des betrachteten Syntaxons gefordert. Eine gemeinsame Klassendifferenzialart ist in keiner Rangstufe Charakterart, erfüllt jedoch das Differenzialartkriterium für mehrere Klassen und grenzt diese folglich gegenüber allen anderen Klassen ab (DENGLER 2003: 82 ff.). Sippen, bei denen es zu vermuten war, dass sich deren Vorkommen nicht nur auf eine Klasse beschränkt, habe ich bezüglich ihres soziologischen Verhaltens nach OBERDORFER (2001) bewertet. Eine Charakterart erfüllt das Differenzialartkriterium gegenüber sämtlichen Syntaxa gleichen Ranges innerhalb desselben Strukturtyps (DENGLER 2003:96). Ich habe dabei die drei Strukturtypen Gehölz-, Kraut- und Kryptogamenvegetation gemäß BERGMEIER et al. (1990) unterschieden. Eine transgressive Charakterart ist zusätzlich in einem übergeordneten Syntaxon Charakterart (DENGLER 2003: 98). Die Stetigkeiten für Syntaxa oberhalb der Assoziation werden als arithmetisches Mittel der Stetigkeiten in den zugehörigen Assoziationen berechnet (1. c.: 83).

Auf diese Weise habe ich die auf Saaremaa vorkommenden Trockenrasengesellschaften voneinander unterschieden. Bei den im Text und in den Tabellen aufgeführten bzw. kenntlich gemachten Sippen handelt es sich somit ausschließlich um territorial gültige Charakter- und Differenzialarten.

Arten, die das Differenzialartenkriterium erfüllen, jedoch von mir nicht bis zur Artebene bestimmt wurden (,spec.-Arten“, zumindest solange es in der jeweiligen Gattung auch weitere bis zur Art bestimmte Proben gab), bei deren Bestimmung ich mir unsicher war (,cf.- 
Arten", die nur teilweise zweifelsfrei bestimmt werden konnten; z. B. Cladonia rei und C. cf. rei) oder Artengruppen (,agg.-Arten“), bei denen ich eine der Kleinarten bestimmt habe (z. B. Cladonia pyxidata agg. und C. novochlorophaea), wurden zu den „Sonstigen“ gestellt.

\subsubsection{Nomenklatur der Syntaxa}

Die Nomenklatur der Syntaxa richtet sich nach DENGLER (2003; Koelerio-Corynephorenea) sowie Dengler \& LÖBEL (2006; Sedo-Scleranthenea). Die höheren Syntaxa der Klasse Festuco-Brometea habe ich nach DENGLER (2004b) benannt, wobei ich in diesem Falle auf die Zuordnung $\mathrm{zu}$ bestimmten Vegetationstypen verzichtet und die Bestände formlos als „Gesellschaft" bezeichnet habe.

\subsubsection{Darstellung der Tabellen}

Die unterschiedenen Syntaxa sind hierarchisch geordnet. Für die unterschiedlichen Hierarchieebenen verwendete ich die in Tab. 11 aufgezählten Abkürzungen:

Tab. 11: Abkürzungen der syntaxonomischen Hierarchieebenen.

$\begin{array}{ll}\text { Hauptgruppe (Klasse bzw. Unterklasse) } & \text { Großbuchstabe (A., B., C.) } \\ \text { Ordnung } & \text { Kleinbuchstabe (a, b, c) } \\ \text { Verband } & \text { Ziffer (1) } \\ \text { Unterverband } & \text { Kleinbuchstabe (a, b) }\end{array}$

Assoziation

A, B oder C (entsprechend der zugehörigen Hauptgruppe) mit fortlaufender Nummerierung innerhalb der Hauptgruppen

Untereinheit

Kleinbuchstabe $(a, b, c)$ hinter dem Assoziationskürzel

Im Tabellenkopf sind die Anzahl der Assoziationen und Aufnahmen sowie die mittlere Gesamtartenzahl und die der Artengruppen ${ }^{1}$ getrennt (arithmetisches Mittel) für das jeweilige Syntaxon angegeben.

Innerhalb eines Syntaxons werden, falls vorhanden, zunächst die territorialen Charakterarten (C) und danach die Differenzialarten (D; d bei Differenzialarten unterhalb des Assoziationsniveaus) aufgeführt.

Zentralsyntaxa sind mit einem Sternchen (*) gekennzeichnet.

Die unter den „Sonstigen“ aufgeführten Sippen sind in keinem Syntaxon Charakter- oder Differenzialarten oder sie besitzen eine sehr breite ökologische Amplitude, wodurch sie nicht sinnvoll als Charakter- oder Differenzialarten einsetzbar sind. Sie sind getrennt nach Artengruppe und Strukturstraten angegeben.

\section{Gesamtstetigkeitstabelle}

Für jede Sippe ist ein gerundeter prozentualer Stetigkeitswert angegeben. Eine „,“ gibt an, dass der Wert unter 0,5\% liegt, ein Punkt hingegen, dass die Art in den entsprechenden Aufnahmen nicht vorkommt. Dunkelgrau unterlegte Stetigkeitswerte verdeutlichen Charakter-arten, hellgraue stehen für eine Klassendifferenzialart bzw. die obere Ebene einer transgressiven Charakterart. Einfach umrahmte Werte kennzeichnen eine Differenzialart. Eine

\footnotetext{
${ }^{1}$ Der Begriff „Artengruppen“ wird im Folgenden für die taxonomischen Artengruppen (Gefäßpflanzen, Moose, Flechten) verwendet.
} 
Spalte wird kursiv abgebildet, wenn das jeweilige Syntaxon durch weniger als zehn Vegetationsaufnahmen repräsentiert ist und somit nur eine eingeschränkte Beurteilung der Charakter- und Differenzialarten möglich ist. $\mathrm{Zu}$ den „Sonstigen“ wurden Arten mit einer Gesamtstetigkeit von mindestens $15 \%$ gestellt. Territoriale Charakterarten sind ab einer prozentualen Stetigkeit von $2 \%$, Differenzialarten ab $5 \%$ dargestellt.

\section{Einzeltabellen der Assoziationen}

Es werden die Vegetationsaufnahmen der jeweiligen Assoziation nebeneinander aufgereiht. Eine Untereinheit differenzierende Sippen sind mit „d“ gekennzeichnet. Die Artenblöcke sind in den Spalten eingerahmt. Am Ende der Einzeltabellen sind Arten, die nur ein- oder zweimal in der betreffenden Assoziation vorkommen, Platz sparender Weise in Textform aufgezählt.

\subsubsection{Diagnostische Artenkombination einer Gesellschaft}

In Abschnitt 4.1 wird zu jeder Gesellschaft die diagnostische Artenkombination angegeben, um einen möglichst detailgetreuen Eindruck der Artenzusammensetzung zu verschaffen. In DENGLER (2003: 162 f.) werden Vorschläge zur Angabe der diagnostischen Artenkombination unterbreitet. In Anlehnung daran habe ich sämtliche territorialen Charakterarten einer Gesellschaft, selbst wenn sie nur eine sehr geringe Stetigkeit besitzen, angegeben und diese fett gesetzt. Zudem sind epigäische Sippen, die mit einer Mindeststetigkeit von $30 \%$ vorkommen (also ungefähr in einem Drittel der Bestände), nach Gefäßpflanzen- und Kryptogamenschicht getrennt in alphabetischer Reihenfolge aufgeführt.

\subsection{Statistische Auswertung}

Für die Vergleiche der untersuchten Parameter habe ich das arithmetische Mittel verwendet. Bei Parametern mit zu stark ungleich verteilten Werten (Standardabweichung höher als das arithmetische Mittel) habe ich mich hingegen für den Median entschieden.

Für die im Folgenden erläuterten statistischen Auswertungsverfahren verwendete ich SPSS (Version 12.0G für Windows).

Der Vergleich der abiotischen und biotischen Parameter, der Artenzahlen sowie der Anteile der Artengruppen am Gesamtartenreichtum auf $4 \mathrm{~m}^{2}$ in den unterschiedlichen Trockenrasengesellschaften wurde mithilfe von Post-hoc-Mehrfachvergleichen (Einfaktorielle ANOVA: Tukey-HSD-Test) durchgeführt.

Zur Untersuchung der Zusammenhänge zwischen der Phythodiversität und abiotischen Parametern sowie der Strukturparameter habe ich aufgrund der räumlichen Nähe einiger Vegetationsaufnahmen zueinander (Vorkommen innerhalb $100 \mathrm{~m}^{2}$ ) nur insgesamt $167 \mathrm{der}$ Vegetationsaufnahmen in die statistische Auswertung einbezogen (jeweils eine Aufnahme der in den $100 \mathrm{~m}^{2}$ befindlichen Aufnahmen zufällig ausgewählt).

Die Zusammenhänge zwischen den Deckungswerten der Vegetation sowie wichtiger Strukturparameter habe ich mittels bivariater Korrelation (Pearson-Korrelationskoeffizient) mit den Signifikanzschwellen $p<0,01$ (hochsignifikant) bzw. $p<0,05$ (signifikant) getestet.

Um zu testen, welchen Einfluss die abiotischen Parameter auf den Gesamtartenreichtum haben, und ob der Artenreichtum der Artengruppen in unterschiedlicher Weise beeinflusst wird, habe ich eine Korrelationsmatrix (Spearman-Rangkorrelation) mit den Signifikanzschwellen $p<0,01$ bzw. $p<0,05$ auf Basis sämtlicher abiotischer Umweltparameter sowie der Artendichten auf $4 \mathrm{~m}^{2}$ (insgesamt und Artengruppen getrennt) erstellt (Tab. 19). Nach der visuellen Überprüfung der Normalverteilung anhand von Histogrammen und Q-Q-TestDiagrammen habe ich jene Variablen eliminiert, die zum einen stark mit einer oder mehreren 
weiteren Variablen co korreliert waren (vgl. Tab. 19; $p \geq 0,8$ ) und zum anderen zu stark von einer für weitere Tests notwendigen Normalverteilung abwichen. Die auf diese Weise herauskristallisierten Variablen habe ich für die folgenden Regressionsanalysen verwendet. In einigen Fällen zeichnete sich kein linearer Zusammenhang zur Artendichte, sondern ein unimodaler Funktionsverlauf ab. Dann habe ich zusätzlich die quadratischen Terme der Variablen in die Regressionsanalysen aufgenommen. Als Analysemethode habe ich schrittweise multiple lineare Regressionsanalysen durchgeführt und dabei die von SPSS vorgegebenen Einstellungen verwendet, bei welchen die Variablen in Abhängigkeit des Signifikanzniveaus ihres F-Wertes eingeschlossen $(p<0,05)$ oder entfernt $(p>0,1)$ werden. In den Regressionsmodellen werden jeweils die Gesamtartenzahlen und die Gruppenartenzahlen als abhängige Variablen zu den abiotischen Parametern als unabhängige Variablen in Beziehung gesetzt.

Um feststellen zu können, ob Parameter, welche nicht in die multiplen Regressionsmodelle eingeschlossen wurden, dennoch signifikante Zusammenhänge zu den Gesamt- oder Gruppenartendichten aufweisen, habe ich zusätzlich einfache Regressionsstatistiken zur Kurvenanpassung durchgeführt. Ich habe dabei zunächst die korrigierten $r^{2}$-Werte des linearen mit jenen des quadratischen Modells verglichen und das Modell mit dem höchsten Wert ausgewählt. Zudem habe ich die Regressionskoeffizienten $b_{0}$ (Konstante) und $b_{1}$ (linearer Term), bei Auswahl des quadratischen Modells zusätzlich $b_{2}$ (quadratischer Term) angegeben, um die Teilmodelle miteinander vergleichen zu können.

Zur Klärung der Frage, welchen Einfluss die biotischen Parameter auf den Gesamtartenreichtum haben, und ob sich daraus Indizien für eine interspezifische Konkurrenz ableiten lassen, habe ich die Strukturparameter einzeln mit den Rest-Varianzen, den Residuen, der multiplen linearen Regressionsanalysen korreliert. 


\section{Ergebnisse}

Im Folgenden werden zunächst die unterschiedlichen Trockenrasengesellschaften der Insel Saaremaa bezüglich floristischer Zusammensetzung, Ökologie, Untergliederung und Verbreitung im Untersuchungsgebiet charakterisiert (Abschnitt 4.1). Des Weiteren werden die Gesellschaften untereinander hinsichtlich der Ökologie und Phytodiversität verglichen (Abschnitt 4.2) und abschließend die Muster der Phytodiversität unter Betrachtung der abiotischen und biotischen Parameter untersucht (Abschnitt 4.3).

\subsection{Charakterisierung der Pflanzengesellschaften}

Auf der Insel Saaremaa konnten insgesamt sieben Trockenrasengesellschaften unterschieden werden (vgl. Tab. 40). Ihre floristischen Gemeinsamkeiten und Unterschiede sind in der beiliegenden Stetigkeitstabelle (Tab. A) dargestellt.

Im Folgenden werden die verschiedenen Trockenrasengesellschaften hinsichtlich ihrer diagnostischen Artenkombination, Untereinheiten, ökologischen Standortbedingungen, ihres Vorkommens im Untersuchungsgebiet, angrenzenden Vegetationstypen sowie ihrer gegenwärtigen Nutzung charakterisiert.

\subsubsection{Caricetum arenariae Christiansen 1927}

\section{Diagnostische Artenkombination}

Gefäßpflanzenschicht: Carex arenaria, Hieracium umbellatum

Kryptogamenschicht: Cephaloziella divaricata, Ceratodon pupureus, Racomitrium canescens, Cetraria aculeata, Cladonia arbuscula, C. crispata var. crispata, C. cornuta, C. deformis, C. fimbriata, C. floerkeana, C. furcata, C. glauca, C. gracilis, C. phyllophora, C. pleurota, C. pyxidata agg., C. rangiferina, C. subulata, C. sulphurina, C. verticillata, Placynthiella oligotropha, Racomitrium canescens

Tab. 12: Mittlere Gesamt- und Gruppenartenzahlen sowie die Deckungsgrade des Caricetum arenariae und seiner Untereinheiten $(n=$ Anzahl der Aufnahmen, $\varnothing=$ arithmetisches Mittel, Min. $=$ Minimum, Max. = Maximum, $\mathrm{s}=$ Standardabweichung).

\begin{tabular}{|c|c|c|c|c|c|c|c|c|c|}
\hline & \multicolumn{4}{|c|}{ Caricetum arenariae } & A1a & \multicolumn{4}{|c|}{ A1b } \\
\hline & \multicolumn{4}{|c|}{$n=6$} & $n=1$ & \multicolumn{4}{|c|}{$n=5$} \\
\hline & $\varnothing$ & Min. & Max. & $\mathrm{s}$ & $\varnothing$ & $\varnothing$ & Min. & Max. & $\mathrm{s}$ \\
\hline Gesamtartenzahl & 14,7 & 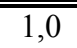 & 24,0 & $\overline{8,3}$ & 1,0 & 17,4 & $\overline{99,0}$ & 24,0 & $\overline{5,5}$ \\
\hline Artenzahl Gefäßpflanzen & 1,5 & 1,0 & 3,0 & 0,8 & 1,0 & 1,6 & 1,0 & 3,0 & 0,9 \\
\hline Artenzahl Moose & 2,3 & 0,0 & 5,0 & 1,8 & 0,0 & 2,8 & 1,0 & 5,0 & 1,5 \\
\hline Artenzahl Flechten & 10,8 & 0,0 & 17,0 & 6,3 & 0,0 & 13,0 & 7,0 & 17,0 & 3,8 \\
\hline Gesamtdeckung Vegetation & 68 & 4 & 96 & 38 & 4 & 81 & 40 & 96 & 23 \\
\hline Deckung Krautschicht & 30 & 4 & 45 & 15 & 4 & 35 & 20 & 45 & 10 \\
\hline Deckung Kryptogamen ges. & 61 & 0 & 95 & 41 & 0 & 74 & 20 & 95 & 31 \\
\hline Deckung Moose & 30 & 0 & 45 & 20 & 0 & 36 & 10 & 45 & 15 \\
\hline Deckung Flechten & 48 & 0 & 75 & 32 & 0 & 57 & 15 & 75 & 24 \\
\hline
\end{tabular}

\section{Charakterisierung}

Das Caricetum arenariae ist sehr arm an Gefäßpflanzenarten. Lediglich Carex arenaria kommt in sämtlichen Aufnahmen mit Deckungsgraden bis $45 \%$ vor. Vereinzelt sind Hieracium umbellatum und Leymus arenarius eingestreut. Bezeichnend für die Gesellschaft 
ist die in vielen Fällen gut ausgeprägte Kryptogamenschicht. Strauchflechten der Gattung Cladonia und Cetraria aculeata kommen hochstet vor und bilden gemeinsam mit Cephaloziella divaricata, Ceratodon purpureus und Racomitrium canescens dichte Bestände (Tab. B).

\section{Untereinheiten}

Auf Saaremaa lassen sich zwei Ausbildungen des Caricetum arenariae unterscheiden: die artenarme (A1a) und die strauchflechtenreiche Ausbildung (A1b) (Tab. B). Bei der artenarmen Ausbildung erreicht Carex arenaria als einzige vorkommende Art eine Deckung von nur $4 \%$ (Tab. 12). In den Beständen der strauchflechtenreichen Ausbildung sind zahlreiche Kryptogamenarten am Vegetationsaufbau beteiligt. Sie bilden mit einem durchschnittlichen Deckungsgrad von $74 \%$ den Hauptanteil der Vegetation. Flechten stellen außerdem mit durchschnittlich 13 Arten auf $4 \mathrm{~m}^{2}$ etwa $75 \%$ der Gesamtartenzahl.

\section{Ökologische Standortbedingungen}

Das Caricetum arenariae kommt auf humusarmen, jedoch basenreichen und tiefgründigen reinen Sandböden der Dünen vor.

Die strauchflechtenreiche Ausbildung des Caricetum arenariae kommt auf bereits entkalkten Böden mit einem mittleren $\mathrm{pH}$-Wert von 5,4 vor. Standorte der artenarmen Ausbildung weisen einen $\mathrm{pH}$-Wert von 8,0 auf. Sie sind mäßig carbonatreich und haben dadurch eine höhere Kationenaustauschkapazität als jene der strauchflechtenreichen Ausbildung (Tab. 21).

\section{Vorkommen im Untersuchungsgebiet, angrenzende Vegetationstypen und Nutzung}

Die strauchflechtenreiche Ausbildung des Caricetum arenariae konnte im äußersten Nordwesten auf einer von Kiefernforst umgebenen Binnendüne westlich von Metsa verzeichnet werden. Das Gebiet befindet sich innerhalb des Vilsandi Nationalparks und ist ungenutzt. An einigen offeneren Stellen waren Fahrspuren zu erkennen.

Die artenarme Ausbildung ist nur mit einer Aufnahme in unmittelbarer Nähe zu einem Badestrand bei Murika im Norden Saaremaas repräsentiert. Der Bestand unterliegt einer sehr hohen Trittbelastung durch Badegäste. An weniger gestörten Bereichen schließt die Gesellschaft an das Festucetum polesicae (Abschnitt 4.1.3) an, welches in Richtung Küste Kontakt zu einer Weißdüne mit Strandhaferfluren und weiter landeinwärts zu einem Kiefernforst hat.

\subsubsection{Helichryso arenarii-Jasionetum litoralis Libbert 1940}

\section{Diagnostische Artenkombination}

Gefäßpflanzenschicht: Androsace septentrionalis, Artemisia campestris, Carex arenaria, Galium verum, Hieracium umbellatum, Pimpinella nigra, Pulsatilla pratensis ssp. pratensis, Sedum acre, Thymus serpyllum

Kryptogamenschicht: Cephaloziella divaricta, Ceratodon pupureus, Cetraria aculeata, C. islandica, Cladonia arbuscula, C. foliacea, C. furcata, C. glauca, C. phyllophora, C. pyxidata agg., Hypogymnia physodes, Placynthiella oligotropha, Racomitrium canescens, Tortula ruraliformis 
Tab. 13: Mittlere Gesamt- und Gruppenartenzahlen sowie die Deckungsgrade des Helichryso-Jasionetum und seiner Untereinheiten $(n=$ Anzahl der Aufnahmen, $\varnothing=$ arithmetisches Mittel, Min. $=$ Minimum, Max. = Maximum, $\mathrm{s}=$ Standardabweichung).

\begin{tabular}{|c|c|c|c|c|c|c|c|c|c|c|c|c|}
\hline & \multicolumn{4}{|c|}{ Helichryso-Jasionetum } & \multicolumn{4}{|c|}{ A2a } & \multicolumn{4}{|c|}{ A2b } \\
\hline & \multicolumn{4}{|c|}{$n=12$} & \multicolumn{4}{|c|}{$n=8$} & \multicolumn{4}{|c|}{$n=4$} \\
\hline & $\varnothing$ & Min. & Max. & $\mathrm{s}$ & $\varnothing$ & Min. & Max. & $\mathrm{S}$ & $\varnothing$ & Min. & Max. & $\mathrm{s}$ \\
\hline Gesamtartenzahl & 21,6 & $7,7,0$ & 31,0 & 6,4 & 20,8 & $7 \overline{7,0}$ & 31,0 & 7,1 & 23,3 & 18,0 & 29,0 & $\overline{5,1}$ \\
\hline Artenzahl Gefäßpflanzen & 8,1 & 4,0 & 16,0 & 3,8 & 7,3 & 4,0 & 12,0 & 3,1 & 9,8 & 4,0 & 16,0 & 4,9 \\
\hline Artenzahl Moose & 3,6 & 0,0 & 7,0 & 1,9 & 3,8 & 0,0 & 7,0 & 2,1 & 3,3 & 2,0 & 5,0 & 1,5 \\
\hline Artenzahl Flechten & 9,9 & 2,0 & 16,0 & 5,2 & 9,8 & 2,0 & 16,0 & 5,4 & 10,3 & 2,0 & 14,0 & 5,6 \\
\hline Gesamtdeckung Vegetation & 66 & 22 & 99 & 25 & 77 & 22 & 99 & 24 & 45 & 35 & 59 & 7 \\
\hline Deckung Krautschicht & 30 & 15 & 50 & 11 & 32 & 20 & 50 & 10 & 25 & 15 & 40 & 12 \\
\hline Deckung Kryptogamen ges. & 53 & 3 & 94 & 34 & 67 & 3 & 94 & 29 & 25 & 5 & 60 & 24 \\
\hline Deckung Moose & 32 & 0 & 75 & 23 & 39 & 0 & 75 & 24 & 19 & 4 & 40 & 15 \\
\hline Deckung Flechten & 31 & 1 & 75 & 26 & 41 & 3 & 75 & 25 & 10 & 1 & 30 & 14 \\
\hline
\end{tabular}

\section{Charakterisierung}

In dieser Gesellschaft werden jene Bestände zusammengefasst, die dem Festucetum polesicae (Abschnitt 4.1.3) bezüglich ihrer Artenzusammensetzung nahe stehen. Jedoch sind sie durch das Fehlen der Assoziationskennarten Alyssum montanum ssp. gmelinii, Dianthus arenarius, Festuca polesica, Koeleria glauca, Cetraria ericetorum gekennzeichnet sind. Außerdem wurden Aufnahmen, die ein spätes Sukzessionsstadium des Caricetum arenariae erfassen, jedoch aufgrund ihres Gefäßpflanzenartenreichtums (z. B. Galium verum, Thymus serpyllum) diesem nicht mehr sinnvoll zugeordnet werden konnten, im Helichryso-Jasionetum eingereiht. Androsace septentrionalis und Cladonia foliacea haben auf Saaremaa ihren Schwerpunkt in dieser Gesellschaft (Tab. A, Tab. C).

\section{Untereinheiten}

Auf Saaremaa lassen sich zwei Untereinheiten des Helichryso-Jasionetum unterscheiden:

Die Hieracium umbellatum-Ausbildung (A2a) ist durch das dominante Auftreten von Strauchflechten aus den Gattungen Cetraria und Cladonia gekennzeichnet. Magerkeitszeiger (z. B. Hieracium umbellatum, Placynthiella oligotropha) und Azidophyten (z. B. Dicranum scoparium, Hypogymnia physodes) sind vertreten. Brachythecium albicans differenziert zusammen mit Festuca rubra ssp. arenaria und Honckenya peploides (Tab. C). Kryptogamen sind mit $67 \%$ am Vegetationsaufbau beteiligt, wobei Moose $39 \%$ und Flechten $41 \%$ der Fläche abdecken (Tab. 13).

Insbesondere durch das Vorkommen zahlreicher Gesteinsflechten und vereinzelter SedoScleranthenea-Arten ist eine Abgrenzung der Verrucaria spp.-Potentilla tabernaemontaniAusbildung (A2b) gegenüber der Hieracium-Ausbildung gegeben (Tab. C). Im Vergleich zur Hieracium-Ausbildung, welche eine mittlere Gesamtdeckung von $77 \%$ aufweist, ist die Vegetation mit einem Deckungsgrad von $45 \%$ recht lückig. Außerdem ist in der VerrucariaPotentilla-Ausbildung mit $25 \%$ ein deutlich geringerer Bedeckungsgrad der Kryptogamenschicht (Moose 19\%, Flechten $10 \%$ ) zu verzeichnen (Tab. 13). Gesteine decken 27,5\% (Median) der Fläche ab, wobei $15 \%$ (Median) deren Oberfläche mit Kryptogamen bewachsen ist (Tab. 25). 


\section{Ökologische Standortbedingungen}

Mit Ausnahme des Bodens der Aufnahme X16 - ein mariner Sand, der aufgrund seines Muschelkalk-Anteils mäßig carbonathaltig ist - sind die Böden der übrigen Aufnahmen carbonatarm bis sehr carbonatarm, jedoch basenreich und humusarm. Die Bestände der Verrucaria-Potentilla-Ausbildung (Aufnahme T05 ausgenommen) kommen auf stark kiesigem Grobsand vor (Median Gesteinsanteil 4,5), wodurch sich die etwas geringere Tiefgründigkeit erklären lässt. Die Hieracium-Ausbildung ist auf nahezu gesteinsfreien Böden verbreitet (Tab. 21).

\section{Vorkommen im Untersuchungsgebiet, angrenzende Vegetationstypen und Nutzung}

Die Vorkommen der Hieracium-Ausbildung konzentrieren sich auf die Sandgebiete im äußersten Nordwesten der Insel westlich von Metsa. Sie kommt dort neben der bereits in den beiden vorherigen Abschnitten erwähnten Binnendüne auf den Graudünen an den Meeresbuchten Uudepanga laht und Haagi lõugas vor. Dort schließen sie an Strandhaferfluren auf den parallel zur Küstenlinie verlaufenden Weißdünen und an die zwischen den Buchten befindliche Sandfläche mit der folgenden Ausbildung an.

Die Verrucaria-Potentilla-Ausbildung besiedelt die zwischen den Meeresbuchten Uudepanga laht und Haagi lõugas liegende offene Sandfläche. Außerdem wächst die Ausbildung an einen Kiefernforst bei Kehila angrenzend sowie bei Katri pank im Südwesten an einer Erosionsfläche der (Steil-)Küste zwischen Strandhaferfluren.

Derzeit ist keine Nutzung der Flächen ersichtlich. Die Küstengebiete im Nordwesten der Insel wurden in der Vergangenheit als militärisches Übungsgelände genutzt.

\subsubsection{Festucetum polesicae Regel 1928}

\section{Diagnostische Artenkombination}

Gefäßpflanzenschicht: Alyssum montanum ssp. gmelinii, Artemisia campestris, Carex arenaria, Cerastium semidecandrum, Dianthus arenarius, Festuca polesica, Galium album ssp. album, G. verum, Hieracium umbellatum, Koeleria glauca, Pulsatilla pratensis ssp. pratensis, Sedum acre, Thymus serpyllum

Kryptogamenschicht: Brachythecium albicans, Cephaloziella divaricata, Ceratodon pupureus, Cetraria aculeata, C. ericetorum, C. islandica, Cladonia furcata, C. pocillum, C. pyxidata agg., C. subulata, Ditrichum flexicaule, Hypogymnia physodes, Peltigera rufescens, Placynthiella oligotropha, Racomitrium canescens, Thuidium abietinum, Tortella inclinata, Tortula ruraliformis 
Tab. 14: Mittlere Gesamt- und Gruppenartenzahlen sowie die Deckungsgrade des Festucetum polesicae und seiner Untereinheiten $(n=$ Anzahl der Aufnahmen, $\varnothing=$ arithmetisches Mittel, Min. $=$ Minimum, Max. = Maximum, $\mathrm{s}=$ Standardabweichung).

\begin{tabular}{|c|c|c|c|c|c|c|c|c|c|c|c|c|c|c|c|c|}
\hline & \multicolumn{4}{|c|}{ Festucetum polesicae } & \multicolumn{4}{|c|}{ A3a } & \multicolumn{4}{|c|}{$\mathbf{A} 3 \mathbf{b}$} & \multicolumn{4}{|c|}{ A3c } \\
\hline & \multicolumn{4}{|c|}{$n=43$} & \multicolumn{4}{|c|}{$n=6$} & \multicolumn{4}{|c|}{$n=12$} & \multicolumn{4}{|c|}{$n=25$} \\
\hline & $\varnothing$ & Min. & Max. & $\mathrm{s}$ & $\varnothing$ & Min. & Max. & $\mathrm{s}$ & $\varnothing$ & Min. & Max. & $\mathrm{s}$ & $\varnothing$ & Min. & Max. & $\mathrm{s}$ \\
\hline Gesamtartenzahl & 23,1 & 9,0 & 37,0 & $\overline{5,6}$ & 18,2 & 12,0 & 26,0 & 6,0 & 24,2 & 17,0 & 33,0 & $\overline{4,6}$ & 23,7 & 9,0 & 37,0 & $\overline{5,6}$ \\
\hline Artenzahl Gefäßpflanzen & 9,7 & 3,0 & 23,0 & 4,7 & 7,0 & 4,0 & 8,0 & 1,5 & 8,4 & 3,0 & 22,0 & 5,0 & 10,9 & 4,0 & 23,0 & 4,8 \\
\hline Artenzahl Moose & 5,8 & 1,0 & 9,0 & 1,8 & 4,5 & 4,0 & 6,0 & 0,8 & 6,8 & 4,0 & 9,0 & 1,3 & 5,6 & 1,0 & 8,0 & 1,9 \\
\hline Artenzahl Flechten & 7,7 & 1,0 & 14,0 & 3,2 & 6,7 & 1,0 & 14,0 & 5,3 & 8,9 & 6,0 & 14,0 & 2,6 & 7,3 & 1,0 & 12,0 & 2,7 \\
\hline Gesamtdeckung Vegetation & 72 & 12 & 100 & 22 & 48 & 12 & 80 & 27 & 81 & 60 & 97 & 13 & 73 & 30 & 100 & 21 \\
\hline Deckung Krautschicht & 37 & 10 & 75 & 16 & 31 & 10 & 65 & 20 & 46 & 25 & 65 & 12 & 35 & 10 & 75 & 16 \\
\hline Deckung Kryptogamen ges. & 57 & 2 & 90 & 27 & 27 & 3 & 70 & 24 & 66 & 25 & 90 & 18 & 60 & 2 & 90 & 27 \\
\hline Deckung Moose & 47 & 1 & 90 & 25 & 18 & 3 & 45 & 16 & 50 & 25 & 70 & 18 & 53 & 1 & 90 & 26 \\
\hline Deckung Flechten & 23 & 1 & 70 & 16 & 12 & 1 & 35 & 14 & 27 & 1 & 70 & 19 & 24 & 1 & 60 & 14 \\
\hline
\end{tabular}

\section{Charakterisierung}

Neben den locker stehenden Horsten von Festuca polesica prägt teilweise auch Koeleria glauca die Gesellschaft. Thymus serpyllum und Sedum acre kommen hochstet vor und bereichern durch ihre intensiv gefärbten Blüten den ansonsten etwas trist anmutenden Vegetationstyp. Während an stark wind- oder sonnenexponierten Standorten häufig offener Sandboden zwischen der Vegetation erkennbar ist, werden an geschützteren oder zeitweise beschatteten Bereichen hohe Deckungsgrade, insbesondere der Moosschicht - mit bezeichnenden Arten wie Brachythecium albicans, Ceratodon pupureus, Ditrichum flexicaule, Racomitrium canescens und Tortula ruraliformis - erreicht. Nicht selten sind Strauchflechten aus den Gattungen Cetraria und Cladonia oder die Blattflechte Peltigera rufescens am Vegetationsaufbau beteiligt (Tab. D).

\section{Untereinheiten}

Auf Saaremaa lassen sich drei Untereinheiten des Festucetum polesicae unterscheiden:

Die typische Ausbildung (A3a) ist weitgehend negativ charakterisiert (Tab. D). Die Bestände weisen bei sämtlichen Artengruppen geringere mittlere Deckungsgrade sowie Artenzahlen auf als die folgenden Ausbildungen (Tab. 14)

Die Placynthiella oligotropha-Cladonia subulata-Ausbildung (A3b) ist durch eine sehr eigenartige Mischung aus Säurezeigern wie Cladonia subulata und Placynthiella oligotropha und Basenzeigern wie Bryum caespiticum, Cladonia cariosa, C. symphycarpa und Gypsophila fastigiata differenziert (Tab. D).

Bezeichnende Arten der Koeleria glauca-Ausbildung (A3c) sind Alyssum montanum ssp. gmelinii, Cephaloziella divaricata, Cetraria ericetorum, Cladonia foliacea, C. glauca, Dianthus arenarius, Erophila verna, Galium album ssp. album, Koeleria glauca, Peltigera canina und Trifolium arvense (Tab. D).

\section{Ökologische Standortbedingungen}

Das Festucetum polesicae besiedelt basenreiche Sandböden mit pH-Werten über 7 und einem sehr geringen Humusgehalt von unter $1 \%$. Die typische und die Placynthiella-CladoniaAusbildung kommen auf mäßig carbonatreichen Böden vor. Die Böden der Koeleria-Ausbildung sind carbonatarm (Tab. 21). 


\section{Vorkommen im Untersuchungsgebiet, angrenzende Vegetationstypen und Nutzung}

Die Gesellschaft ist auf Saaremaa in ihrem Vorkommen auf die Sandgebiete in Küstennähe beschränkt.

Die typische und die Placynthiella-Cladonia-Ausbildung sind vorwiegend im Norden und Nordwesten der Insel verbreitet. Sie kommen z. B. auf einem ehemaligen militärischen Übungsgelände östlich von Kõõru, einem Ausläufer der Endmoräne des „West Saaremaa Hochlands" (auf ca. 20-35 m ü. NN), umgeben von Heide und Kiefernwald sowie in der Bucht Pidula laht südwestlich von Murika zwischen Kiefernforsten und Strandhaferfluren vor.

Die Koeleria-Ausbildung hat ihren Verbreitungsschwerpunkt im Süden der Insel. Sie ist im Dünengebiet von Mändjala bis Tehumardi teilweise an Röhrichte oder Strandhaferfluren angrenzend, ansonsten im Mosaik mit Kiefernforsten $\mathrm{zu}$ finden. Auf dem ehemaligen Gefechtsfeld bei Tehumardi ist sie mit Helictotrichon pratense dominierten Halbtrockenrasen verzahnt.

Die Flächen sind, abgesehen von den selten auftretenden mechanischen Belastungen durch das Betreten von Badegästen in Küstennähe, praktisch ungenutzt. Einzig das ehemalige Gefechtsfeld bei Tehumardi wird durch eine gelegentliche Pflegemahd offen gehalten.

\subsubsection{Sileno otitae-Festucetum brevipilae Libbert 1933 corr. Kratzert \& Dengler 1999}

\section{Diagnostische Artenkombination}

Gefäßpflanzenschicht: Achillea millefolium ssp. millefolium, Agrostis capillaris, Arenaria serpyllifolia ssp. glutinosa, Artemisia campestris, Cerastium semidecandrum, Elymus repens ssp. repens, Festuca ovina, F. rubra ssp. rubra, Galium verum, Helictotrichon pratense, Luzula campestris, Medicago lupulina, Phleum phleoides, Pimpinella nigra, Plantago lanceolata, Poa angustifolia, Potentilla argentea agg., Sedum acre, Thymus serpyllum, Trifolium arvense

Kryptogamenschicht: Brachythecium albicans, Ceratodon pupureus, Cladonia furcata, C. pyxidata agg., Climacium dendroides, Ditrichum flexicaule, Homalothecium lutescens, Hypnum cupressiforme var. lacunosum, Peltigera canina, $P$. rufescens, Thuidium abietinum

Tab. 15: Mittlere Gesamt- und Gruppenartenzahlen sowie die Deckungsgrade des Sileno-Festucetum und seiner Untereinheiten $(n=$ Anzahl der Aufnahmen, $\varnothing=$ arithmetisches Mittel, Min. $=$ Minimum, Max. $=$ Maximum, $\mathrm{s}=$ Standardabweichung).

\begin{tabular}{|c|c|c|c|c|c|c|c|c|c|c|c|c|}
\hline & \multicolumn{4}{|c|}{ Sileno-Festucetum } & \multicolumn{4}{|c|}{$\mathbf{A 4 a}$} & \multicolumn{4}{|c|}{ A4b } \\
\hline & \multicolumn{4}{|c|}{$n=24$} & \multicolumn{4}{|c|}{$n=13$} & \multicolumn{4}{|c|}{$n=11$} \\
\hline & $\varnothing$ & Min. & Max. & $\mathrm{s}$ & $\varnothing$ & Min. & Max. & $\mathrm{s}$ & $\varnothing$ & Min. & Max. & $\mathrm{s}$ \\
\hline Gesamtartenzahl & 30,4 & 20,0 & 48,0 & 6,7 & 31,9 & 20,0 & 48,0 & 4,8 & 28,6 & 23,0 & 37,0 & 4,8 \\
\hline Artenzahl Gefäßpflanzen & 21,0 & 13,0 & 33,0 & 4,9 & 20,1 & 13,0 & 33,0 & 4,3 & 22,0 & 17,0 & 29,0 & 4,3 \\
\hline Artenzahl Moose & 5,3 & 0,0 & 12,0 & 2,2 & 5,5 & 3,0 & 12,0 & 2,1 & 5,2 & 0,0 & 8,0 & 2,1 \\
\hline Artenzahl Flechten & 4,1 & 0,0 & 17,0 & 4,1 & 6,4 & 2,0 & 17,0 & 2,0 & 1,5 & 0,0 & 5,0 & 2,0 \\
\hline Gesamtdeckung Vegetation & 89 & 35 & 100 & 17 & 97 & 80 & 100 & 6 & 80 & 35 & 99 & 21 \\
\hline Deckung Krautschicht & 65 & 15 & 97 & 24 & 68 & 15 & 97 & 26 & 62 & 30 & 89 & 21 \\
\hline Deckung Kryptogamen ges. & 70 & 0 & 98 & 26 & 86 & 60 & 98 & 10 & 50 & 0 & 85 & 27 \\
\hline Deckung Moose & 66 & 0 & 97 & 25 & 80 & 60 & 97 & 11 & 49 & 0 & 83 & 26 \\
\hline Deckung Flechten & 13 & 0 & 50 & 14 & 21 & 2 & 50 & 14 & 4 & 0 & 25 & 8 \\
\hline
\end{tabular}




\section{Charakterisierung}

Das Sileno-Festucetum ist zum einen durch dominant auftretende Arten der KoelerioCorynephoretea wie Artemisia campestris, Brachythecium albicans, Ceratodon purpureus, Potentilla argentea agg., Sedum acre und Trifolium arvense eng an diese Klasse gebunden. Zum anderen sind Halbtrockenrasen- oder Grünlandarten beigemischt, die auf günstigere Bodenverhältnisse hinweisen und die soziologische Nähe zu den Gesellschaften der FestucoBrometea verdeutlichen. Typische mesophile Arten der Festuco-Brometea wie Briza media, Filipendula vulgaris, Fragaria viridis, Galium boreale oder Knautia arvensis fehlen jedoch insgesamt oder sind sehr selten. Die Kryptogamenschicht wird aus wenigen, teilweise hohe Deckungsgrade erreichenden Arten gebildet. Verschiedene Kräuter sorgen aufgrund ihrer unterschiedlichen Blütezeiten für eine abwechslungsreiche Farbgebung während der gesamten Vegetationsperiode (Tab. E).

\section{Untereinheiten}

Auf Saaremaa lassen sich zwei Untereinheiten des Sileno-Festucetum unterscheiden:

Bei der Cladonia furcata-Helictotrichon pratense-Ausbildung (A4a) handelt es sich um sehr dicht geschlossene Bestände mit einer durchschnittlichen Gesamtdeckung von $97 \%$. Die Kryptogamenschicht ist mit einem mittleren Deckungsgrad von $86 \%$ dichter geschlossen als die der Potentilla-Agrostis-Ausbildung. $80 \%$ der Fläche werden dabei von Moosen, $21 \%$ von Flechten abgedeckt. Außerdem liegt die mittlere Flechtenartenzahl mit einem Wert von 6,4 deutlich höher (Tab. 15). Hinsichtlich der Artenzusammensetzung ist die Ausbildung sehr heterogen. Neben charakteristischen Strauchflechten (Cladonia spp., Cetraria spp.), Magerkeits- und Säurezeigern der Sandtrockenrasen (z. B. Carex arenaria, Dicranum scoparium) treten vermehrt Charakterarten der Festuco-Brometea-Halbtrockenrasen wie Helictotrichon pratense, Homalothecium lutescens, Hypnum cupressiforme var. lacunosum, Phleum phleoides und Pimpinella nigra hochstet auf und differenzieren die Ausbildung (Tab. E).

Die Potentilla argentea agg.-Agrostis capillaris-Ausbildung (A4b) ist durch mäßig saure Verhältnisse anzeigende Arten wie Agrostis capillaris, Rumex acetosella und Potentilla argentea agg. differenziert. Auf etwas günstigere Nährstoffverhältnisse weisen Sippen wie Dactylis glomerata ssp. glomerata, Festuca rubra ssp. rubra und Rhythidiadelphus squarrosus hin (Tab. E). Moose decken in den Beständen im Durchschnitt 49 \%, Flechten nur $4 \%$ und damit einen deutlich geringeren Anteil der Fläche als bei der Cladonia furcataHelictotrichon pratense-Ausbildung ab (Tab. 15).

\section{Ökologische Standortbedingungen}

Das Sileno-Festucetum kommt auf carbonatarmen, basenreichen Böden vor, deren pH-Wert im Schnitt bei 6,6 liegt (Tab. 21). Die Cladonia-Helictotrichon-Ausbildung kommt hauptsächlich auf reinen Sandböden vor. Bei der Potentilla-Agrostis-Ausbildung variieren die Bodenarten von reinem Sand über lehmigen Sand bis hin zu sandigem Lehm (Tab. E).

\section{Vorkommen im Untersuchungsgebiet, angrenzende Vegetationstypen und Nutzung}

Das Sileno-Festucetum kommt im Endmoränengebiet, in Kärla sowie südöstlich davon und auf fossilen Strandwällen der Insel Vilsandi vor. Ein weiteres Vorkommen liegt an der Küste im Norden bei Varese Sadam (östlich von Panga pank). Die Bestände grenzen an Kiefernforste, Juniperus-Gebüsche, Halb- und Sandtrockenrasen, Ruderal- oder Ackerflächen sowie Mähwiesen an. Die Flächen der Potentilla-Agrostis-Ausbildung werden teilweise gemäht oder liegen brach. Jene der Cladonia-Helictotrichon-Ausbildung sind durchweg ungenutzt. 


\subsubsection{Cladonio symphycarpiae-Sedetum albi Tx. 1951}

\section{Diagnostische Artenkombination}

Gefäßpflanzenschicht: Acinos arvensis, Achillea millefolium ssp. millefolium, Anthyllis vulneraria ssp. vulneraria, Arenaria sepyllifolia ssp. glutinosa, Arenaria sepyllifolia ssp. serpyllifolia, Artemisia campestris, Bromus hordeaceus ssp. hordeaceus, Cerastium semidecandrum, Dactylis glomerata ssp. glomerata, Echium vulgare, Erophila verna, Festuca rubra ssp. rubra, Galium verum, Hypericum perforatum, Leucanthemum vulgare, Medicago lupulina, Plantago lanceolata, Poa compressa, Potentilla argentea agg., Saxifraga tridactylites, Sedum acre

Kryptogamenschicht: Brachythecium albicans, Bryum caespiticum, Ceratodon purpureus, Ditrichum flexicaule, Homalothecium lutescens, Hypnum cupressiforme var. lacunosum, Peltigera rufescens, Thuidium abietinum, Tortella inclinata

Tab. 16: Mittlere Gesamt- und Gruppenartenzahlen sowie die Deckungsgrade des Cladonio-Sedetum und seiner Untereinheiten ( $n=$ Anzahl der Aufnahmen, $\varnothing=$ arithmetisches Mittel, Min. $=$ Minimum, Max. $=$ Maximum, $\mathrm{s}=$ Standardabweichung).

\begin{tabular}{|c|c|c|c|c|c|c|c|c|c|c|c|c|}
\hline & \multicolumn{4}{|c|}{ Cladonio-Sedetum } & \multicolumn{4}{|c|}{ B1a } & \multicolumn{4}{|c|}{ B1b } \\
\hline & \multicolumn{4}{|c|}{$n=54$} & \multicolumn{4}{|c|}{$n=30$} & \multicolumn{4}{|c|}{$n=14$} \\
\hline & $\varnothing$ & Min. & Max. & $\mathrm{s}$ & $\varnothing$ & Min. & Max. & $\mathrm{s}$ & $\varnothing$ & Min. & Max. & $\mathrm{s}$ \\
\hline Gesamtartenzahl & 31,5 & 16,0 & $\overline{445,0}$ & 8,5 & 33,6 & 22,0 & 45,0 & 7,1 & 28,9 & 16,0 & $\overline{445,0}$ & $\overline{9,6}$ \\
\hline Artenzahl Gefäßpflanzen & 18,5 & 10,0 & 35,0 & 5,5 & 19,9 & 14,0 & 26,0 & 3,5 & 16,6 & 10,0 & 35,0 & 7,1 \\
\hline Artenzahl Moose & 7,5 & 3,0 & 13,0 & 2,5 & 7,9 & 5,0 & 12,0 & 2,1 & 7,0 & 3,0 & 13,0 & 3,0 \\
\hline Artenzahl Flechten & 5,3 & 0,0 & 16,0 & 4,4 & 5,5 & 0,0 & 16,0 & 4,5 & 5,1 & 0,0 & 12,0 & 4,6 \\
\hline Gesamtdeckung Vegetation & 71 & 8 & 99 & 28 & 87 & 70 & 99 & 11 & 51 & 8 & 90 & 30 \\
\hline Deckung Krautschicht & 51 & 10 & 97 & 22 & 57 & 35 & 97 & 18 & 45 & 10 & 90 & 26 \\
\hline Deckung Kryptogamen ges. & 54 & 10 & 98 & 28 & 68 & 35 & 98 & 20 & 36 & 10 & 85 & 27 \\
\hline Deckung Moose & 49 & 2 & 98 & 27 & 59 & 30 & 98 & 21 & 35 & 2 & 85 & 28 \\
\hline Deckung Flechten & 10 & 0 & 60 & 17 & 16 & 0 & 60 & 21 & 2 & 0 & 10 & 3 \\
\hline
\end{tabular}

\section{Charakterisierung}

Das Cladonio-Sedetum ist im Vergleich zum Crepido-Allietum (Abschnitt 4.1.6) floristisch etwas verarmt. Es fehlen in den meisten Fällen die wechselfeuchte Verhältnisse anzeigenden Arten, die bunten Erdflechten und der größte Teil der kleinen akrokarpen Moose. Sedum acre ist im Frühsommer mit seinen gelben Blüten Aspekt bestimmend. Die Moosschicht wird von Polster bildenden Arten wie Ceratodon purpureus und Ditrichum flexicaule beherrscht (Tab. F). Eine Reihe anueller Arten differenzieren gegenüber dem Crepido-Allietum (Tab. A).

\section{Untereinheiten}

Auf Saaremaa lassen sich zwei Untereinheiten des Cladonio-Sedetum unterscheiden:

Die Artemisia campestris-Bryum caespiticum-Ausbildung (B1a) ist durch die Gräser Dactylis glomerata ssp. glomerata, Poa angustifolia und Poa compressa differenziert. Die Thalli von Peltigera spp. erreichen teilweise eine beträchtliche Größe und sind neben Artemisia campestris die bestandsbildenden Arten. Die Bestände der Artemisia-BryumAusbildung sind mit einem mittleren Deckungsgrad der Vegetation von 87 \% im Vergleich zu den übrigen Beständen der Felsgrusgesellschaften relativ dicht (Tab. 25).

Die Tortella tortuosa-Leontodon autumnalis-Ausbildung (B1b) setzt sich aus einem heterogenen Aufnahmeblock zusammen. Sie ist durch Arten wie Agrostis vinealis, Arrhenatherum elatius, Campylium polygamum, Distichium inclinatum, Encalypta 
streptocarpa, Leontodon autumnalis und Tortella tortuosa differenziert. Die Ausbildung hat eine mittlere Gesamtdeckung der Vegetation von $51 \%$ und ist somit im Vergleich zur Artemisia-Bryum-Ausbildung weitaus lückenreicher. Besonders die Flechten decken - bei etwa gleicher Artenzahl - mit nur $2 \%$ einen deutlich geringeren Anteil der Fläche ab (Tab. 16).

\section{Ökologische Standortbedingungen}

Die Gesellschaft kommt auf Saaremaa außerhalb der Alvare vor und besiedelt neben Felsstandorten und fossilen Strandwällen häufig Sekundärstandorte anthropogenen Ursprungs, auf welchen sich die territoriale Charakterart Saxifraga tridactylites ausbreitet und sogar teilweise Deckung erreicht. Die Böden sind carbonatreich und sehr flachgründig. Bei der ArtemisiaBryum-Ausbildung ist Sand die vorherrschende Bodenart, bei der Tortella-LeontodonAusbildung reiner Schluff (Tab. F).

Die Tortella-Leontodon-Ausbildung kommt auf Skelettböden mit einer mittleren Tiefgründigkeit von weniger als $5 \mathrm{~cm}$ vor. Die Bodenverhältnisse der Artemisia-BryumAusbildung sind hingegen mit einem Gesteinsanteil von höchstens $30 \%$ und einer Tiefgründigkeit von 5-10 cm etwas weniger extrem (Tab. 21). Freiliegende Gesteine erreichen in den offenen Beständen der Tortella-Leontodon-Ausbildung eine Deckung von 40\% (Median). Bei der Artemisia-Bryum-Ausbildung sind es lediglich $2 \%$ (Median) (Tab. 25).

\section{Vorkommen im Untersuchungsgebiet, angrenzende Vegetationstypen und Nutzung}

Fossile Strandwälle und Felsstandorte sind hauptsächlich im westlichen Teil sowie im Norden der Insel zu finden. Einige Aufnahmen solcher Standorte stammen aus der Bucht Pidula im Nordwesten und von der Insel Vilsandi. Die angesprochenen Sekundärstandorte sind reichlich im Westen der Insel auf den mittlerweile ungenutzten Militärgeländen (Betonplatten der Bunker, Panzerstraßen, Dächer von Gebäuden, Schuttplätze), auf Industriebrachen oder auch durch Lesesteinwälle vertreten. Im restlichen Teil des Untersuchungsgebietes sind potenzielle Standorte und somit weitere Vorkommen der Gesellschaft zu erwarten. Unterschiedlichste Vegetationstypen schließen an die Bestände an.

\subsubsection{Crepido pumilae-Allietum alvarensis Krahulec et al. ex Dengler \& Löbel 2006}

\section{Diagnostische Artenkombination}

Gefäßpflanzenschicht: Acinos arvensis, Achillea millefolium ssp. millefolium, Agrostis stolonifera, Allium schoenoprasum var. alvarense, Anthyllis vulneraria ssp. vulneraria, Arenaria serpyllifolia ssp. glutinosa, Artemisia campestris, A. rupestris, Asperula tinctoria, Centaurea jacea, Euphrasia stricta, Festuca ovina, Filipendula vulgaris, Galium boreale, G. verum, Helictotrichon pratense, Hieracium pilosella, Hypericum perforatum, Juniperus communis ssp. communis, Linum catharticum, Medicago lupulina, Pimpinella nigra, Plantago lanceolata, Poa compressa, Potentilla tabernaemontani, Pseudolysimachion spicatum, Sagina nodosa, Sedum acre, S. album, Thymus serpyllum

Kryptogamenschicht: Agonimia globulifera, A. tristicula, Bacidia bagliettoana, Barbula convoluta, B. unguiculata, Bryum argenteum, B. caespiticum, Campylium sommerfeltii, Ceratodon purpureus, Cetraria islandica, Cladonia pocillum, C. rangiformis, C. subrangiformis, C. symphycarpia, Crepis tectorum ("outcropForm"), Ctenidium molluscum, Distichum inclinatum, Ditrichum flexicaule, Encalypta streptocarpa, Fissidens dubius, Fulgensia bracteata, Homalothecium lutescens, Hypnum cupressiforme var. lacunosum, Leptogium gelatinosum, Myurella julacea, Nostoc spec., Peltigera rufescens, Psora decipiens, Riccia bifurca, Thuidium abietinum, Toninia sedifolia, Tortella fragilis, T. inclinata, T. tortuosa, Weissia brachycarpa 
Tab. 17: Mittlere Gesamt- und Gruppenartenzahlen sowie die Deckungsgrade des Crepido-Allietum und seiner Untereinheiten $(n=$ Anzahl der Aufnahmen, $\varnothing=$ arithmetisches Mittel, Min. $=$ Minimum, Max. $=$ Maximum, $\mathrm{s}=$ Standardabweichung).

\begin{tabular}{|c|c|c|c|c|c|c|c|c|c|c|c|c|}
\hline & \multicolumn{4}{|c|}{ Crepido-Allietum } & \multicolumn{4}{|c|}{ B2a } & \multicolumn{4}{|c|}{ B2b } \\
\hline & \multicolumn{4}{|c|}{$n=54$} & \multicolumn{4}{|c|}{$n=30$} & \multicolumn{4}{|c|}{$n=14$} \\
\hline & $\varnothing$ & Min. & Max. & $\mathrm{s}$ & $\varnothing$ & Min. & Max. & $\mathrm{s}$ & $\varnothing$ & Min. & Max. & $\mathrm{s}$ \\
\hline Gesamtartenzahl & 47,7 & 23,0 & 71,0 & 11,4 & 47,9 & 23,0 & 71,0 & 11,4 & 47,3 & 30,0 & 70,0 & 11,9 \\
\hline Artenzahl Gefäßpflanzen & 21,4 & 11,0 & 36,0 & 5,4 & 21,7 & 11,0 & 36,0 & 5,2 & 20,6 & 11,0 & 32,0 & 6,0 \\
\hline Artenzahl Moose & 14,1 & 4,0 & 27,0 & 5,6 & 15,3 & 4,0 & 27,0 & 5,3 & 10,9 & 4,0 & 19,0 & 5,6 \\
\hline Artenzahl Flechten & 11,4 & 1,0 & 23,0 & 5,7 & 10,2 & 1,0 & 23,0 & 5,7 & 15,1 & 8,0 & 21,0 & 4,3 \\
\hline Gesamtdeckung Vegetation & 77 & 25 & 100 & 19 & 79 & 30 & 100 & 18 & 70 & 25 & 99 & 21 \\
\hline Deckung Krautschicht & 56 & 7 & 99 & 20 & 60 & 20 & 99 & 20 & 45 & 7 & 70 & 17 \\
\hline Deckung Kryptogamen ges. & 56 & 2 & 95 & 25 & 57 & 2 & 95 & 25 & 52 & 4 & 95 & 25 \\
\hline Deckung Moose & 43 & 2 & 95 & 24 & 48 & 2 & 95 & 24 & 31 & 4 & 65 & 19 \\
\hline Deckung Flechten & 19 & 0 & 95 & 20 & 15 & 0 & 95 & 19 & 29 & 1 & 65 & 20 \\
\hline
\end{tabular}

\section{Charakterisierung}

Das Crepido-Allietum ist im Untersuchungsgebiet der typische Vegetationstyp der Alvare in einem frühen Sukzessionsstadium. Es handelt sich vorwiegend um relativ offene Flächen mit vereinzelt stehenden Juniperus-Sträuchern. Die Gesellschaft zeichnet sich durch das hochstete Auftreten der territorialen Charakterarten Sedum album, Cladonia symphycarpia und der kontinentalen Steppenart Artemisia rupestris aus. Sie ist durch wechselfeuchte Verhältnisse anzeigende Arten wie Agrostis stolonifera, Ctenidium molluscum, Fissidens dubius, Galium boreale und Linum catharticum differenziert. Neben den häufig vorkommenden bunten Erdflechten Fulgensia bracteata, Psora decipiens und Toninia sedifolia sind stets eine Vielzahl kleiner akrokarper Moosarten vertreten (Tab. A, Tab. G).

\section{Untereinheiten}

Auf Saaremaa lassen sich zwei Untereinheiten des Crepido-Allietum unterscheiden:

Bei der Artemisia rupestris-Ausbildung (B2a) ist das Nebeneinander von hygrophilen und xerophilen Elementen sehr ausgeprägt. Wechselfeuchtezeiger differenzieren die Ausbildung (Tab. F). Bestandsbildend sind vor allem Kräuter mit $60 \%$ und Moose mit 48 \%.

Die Cetraria aculeata-Echium vulgare-Ausbildung (B2b) unterscheidet sich von der Artemisia rupestris-Ausbildung durch das Auftreten einiger Sandtrockenrasenarten wie Cetraria aculeata, Cladonia foliacea und C. gracilis sowie das Fehlen der erwähnten Wechselfeuchtezeiger (Tab. G). Kräuter sind im Schnitt mit $45 \%$ und Moose mit $31 \%$ am Bestandsaufbau beteiligt. Der Deckungsgrad der Flechtenschicht liegt mit $29 \%$ wesentlich höher als bei der Artemisia rupestris-Ausbildung (Tab. 17).

\section{Ökologische Standortbedingungen}

Das Crepido-Allietum besiedelt mäßig carbonatreiche, flachgründige und skelettreiche Rendzinen mit einem 5-10 cm starken humusreichen Oberboden. Die vorherrschenden Bodenarten sind reiner oder sandiger Schluff (Tab. G). Häufig ist ein ausgeprägtes Mikrorelief mit höher liegenden, ständig trockenen Bulten und tiefer gelegenen Senken, die nach starken Regenfällen und bei gefrorenem Boden im Winter durch Tauwasser überstaut werden können, festzustellen. Auf den Bulten wachsen z. B. Festuca ovina und Artemisia campestris und in den Senken die angesprochenen Wechselfeuchtezeiger. Es ist zu beobachten, dass verschiedene Moosarten vorwiegend auf den offenen Bereichen am Fuße der Bulten, welche humusreicher wirken, vorkommen. 
Die Böden der Artemisia-Ausbildung sind im Durchschnitt etwas tiefgründiger und gesteinsärmer als jene der Cetraria-Echium-Ausbildung (Tab. 21).

\section{Vorkommen im Untersuchungsgebiet, angrenzende Vegetationstypen und Nutzung}

Die Gesellschaft ist vor allem in den Alvargebieten im westlichen Teil der Insel sowie östlich von Kuressaare auf dem Alvar bei Iipla verbreitet. Weitere Vorkommen konnten auf Felsstandorten an den Steilküsten im Norden und auf Insel Vilsandi verzeichnet werden. Juniperus-Gebüsche, Kiefernforste und Halbtrockenrasen der Klasse Festuco-Brometea sind als angrenzende Vegetationstypen zu nennen. Die Flächen scheinen momentan ungenutzt zu sein. Es ist allerdings anzunehmen, dass sie als Pflegemaßnahme hin und wieder maschinell offen gehalten werden ${ }^{2}$. Im Nationalpark Vilsandi liegen jedoch relativ große, noch intakte Flächen (z. B. der Atla-Alvar), die der Sukzession überlassen wurden und zunehmend von Juniperus communis überwachsen werden.

\subsubsection{Helictotrichon pratense-[Brachypodietalia pinnati]-Gesellschaft}

\section{Diagnostische Artenkombination}

Gefäßpflanzenschicht: Achillea millefolium ssp. millefolium, Anthyllis vulneraria ssp. vulneraria, Artemisa campestris, Asperula tinctoria, Briza media, Campanula rotundifolia, Carex ericetorum, C. flacca, C. pilulifera, Centaurea jacea, Cirsium acaule, Dactylis glomerata ssp. glomerata, Festuca ovina, F. rubra ssp. rubra, Filipendula vulgaris, Fragaria viridis, Galium boreale, G. verum, Helictotrichon pratense, H. pubescens ssp. pubescens, Hieracium pilosella, Hypericum perforatum, Knautia arvensis, Leucanthemum vulgare, Linum catharticum, Lotus corniculatus ssp. corniculatus, Medicago lupulina, Pimpinella nigra, P. saxifraga, Plantago lanceolata, P. media, Poa angustifolia, P. compressa, Potentilla incana, P. tabernaemontani, P. ×subarenaria, Pseudolysimachion spicatum, Ranunculus bulbosus, Senecio jacobaea ssp. jacobaea, Sesleria caerulea, Solidago virgaurea, Thymus serpyllum, Trifolium montanum

Kryptogamenschicht: Barbula convoluta, Brachythecium rutabulum, Campylium chrysophyllum, Ditrichum flexicaule, Fissidens dubius, Homalothecium lutescens, Hypnum cupressiforme var. lacunosum, Thuidium abietinum, T. philibertii, Tortella tortuosa

Tab. 18: Mittlere Gesamt- und Gruppenartenzahlen sowie die Deckungsgrade der Helictotrichon-Gesellschaft $(n=$ Anzahl der Aufnahmen, $\varnothing=$ arithmetisches Mittel, Min. $=$ Minimum, Max. $=$ Maximum, $\mathrm{s}=$ Standardabweichung).

\begin{tabular}{|l||cccc|}
\hline \multicolumn{1}{|c||}{} & \multicolumn{4}{c|}{ Helictotrichon -Gesellschaft (C1) } \\
\hline \hline \multicolumn{1}{|c||}{} & $\varnothing$ & Min. & Max. & $\mathrm{s}$ \\
Gesamtartenzahl & 40,5 & 21,0 & 60,0 & 8,9 \\
Artenzahl Gefäßpflanzen & 30,5 & 15,0 & 47,0 & 8,3 \\
Artenzahl Moose & 7,5 & 2,0 & 14,0 & 3,0 \\
Artenzahl Flechten & 2,4 & 0,0 & 11,0 & 2,9 \\
\hline Gesamtdeckung Vegetation & 92 & 45 & 100 & 12 \\
Deckung Krautschicht & 83 & 25 & 100 & 17 \\
Deckung Kryptogamen ges. & 66 & 20 & 97 & 23 \\
Deckung Moose & 65 & 20 & 97 & 24 \\
Deckung Flechten & 3 & 0 & 40 & 7 \\
\hline
\end{tabular}

\footnotetext{
${ }^{2}$ In Estland existiert seit dem Jahr 2001 eine Vereinigung namens „Estonian Semi-natural Communities Conservation Association“, die es sich zur Aufgabe gemacht hat, Kulturlandschaften zu pflegen und zu erhalten. Sie wurde im Rahmen des Projektes „10 for nature“ gegründet, welches vom „Eeconet Action Fund“ initiiert wurde (EECONET ACTION FUND 2005).
} 


\section{Charakterisierung}

Helictotrichon pratense prägt in den meisten Fällen das Erscheinungsbild der blumenbunten Gesellschaft. Während der Sommermonate alternieren die Blühphasen unterschiedlicher Kräuter wie Anthyllis vulneraria ssp. vulneraria, Centaurea jacea, Filipendula vulgaris, Hieracium pilosella, Knautia arvensis, Linum catharticum und Potentilla spp., wodurch sich die farblichen Aspekte kontinuierlich wandeln. Häufig sind Arten wie Briza media, Carex flacca, Ctenidium molluscum, Galium boreale, Linum catharticum und Sesleria caerulea beigemischt, deren zweiter Verbreitungsschwerpunkt in den Niedermooren der Klasse ParvoCaricetea den Held \& Westhoff in Westhoff \& den Held 1969 nom. cons. propos. liegt (Tab. H).

Die Bestände der Helictotrichon-Gesellschaft weisen größtenteils eine sehr dicht geschlossene Vegetation auf. Wenige pleurokarpe Moosarten erreichen teilweise hohe Deckungsgrade (Tab. H), Flechten sind mit einer mittleren Deckung von nur $2,7 \%$ zu vernachlässigen (Tab. 18).

\section{Ökologische Standortbedingungen}

Die Helictotrichon-Gesellschaft ist auf mäßig carbonathaltigen, etwas tiefgründigeren Böden (mittlere Tiefgründigkeitsklasse $3=10-25 \mathrm{~cm}$ ) mit einem mittleren $\mathrm{pH}$-Wert von 7,3 verbreitet (Tab. 21). Sandiger Schluff oder Lehm-Böden herrschen vor (Tab. H). Die zahlreichen meso-philen Sippen lassen eine günstigere Nährstoffversorgung und Wasserhaltefähigkeit der Böden vermuten.

\section{Vorkommen im Untersuchungsgebiet, angrenzende Vegetationstypen und Nutzung}

Die Gesellschaft ist im gesamten Untersuchungsgebiet verbreitet und mit unterschiedlichen Vegetationstypen verzahnt. Sie tritt sowohl neben Sandtrockenrasen, Niedermooren, Kiefernforsten oder Juniperus-Gebüschen als auch neben den Felsgrusfluren der Alvare als fortgeschrittenes Sukzessionsstadium auf. Die aufgenommenen Bestände sind größtenteils ungenutzt. Hin und wieder ist eine Beweidung mit Rindern oder Pferden oder eine Mahd der Flächen zu beobachten.

\section{2 Ökologie und Phytodiversität - eine vergleichende Betrachtung der Trockenrasen- gesellschaften Saaremaas}

Die Wechselbeziehungen zwischen den abiotischen Umweltparametern sowie den Gesamtund Gruppenartenzahlen sind in Tab. 19 dargestellt. 
Tab. 19: Korrelationsmatrix (Spearman-Rangkorrelation) der Gesamt- und Gruppenartenzahlen sowie der erfassten abiotischen Umweltparameter $(n=167)$. Hochsignifikante Zusammenhänge $(p<0,01)$ sind fett dunkelgrau, auf dem Niveau $p<0,05$ signifikante Zusammenhänge hellgrau unterlegt.

\begin{tabular}{|c|c|c|c|c|c|c|c|c|c|}
\hline & $\begin{array}{c}\text { Gesamt- } \\
\text { artenzahl }\end{array}$ & $\begin{array}{l}\text { Artenzahl } \\
\text { Gefäß- } \\
\text { pflanzen }\end{array}$ & $\begin{array}{c}\text { Artenzahl } \\
\text { Moose }\end{array}$ & $\begin{array}{c}\text { Artenzahl } \\
\text { Flechten }\end{array}$ & $\begin{array}{c}\text { Humus- } \\
\text { gehalt } \\
{[\%]}\end{array}$ & $\begin{array}{c}\mathrm{CaCO}_{3} \\
\text { (ordinal) }\end{array}$ & pH-Wert & $\begin{array}{c}\text { H-Wert } \\
{[\mathrm{mval} / 100 \mathrm{~g}]}\end{array}$ & $\begin{array}{c}\text { S-Wert } \\
{[\mathrm{mval} / 100 \mathrm{~g}]}\end{array}$ \\
\hline Gesamtartenzahl & 1,000 & 0,794 & 0,656 & 0,203 & 0,548 & 0,179 & 0,003 & $-0,057$ & 0,256 \\
\hline $\begin{array}{l}\text { Artenzahl } \\
\text { Gefäßpflanzen }\end{array}$ & & 1,000 & 0,279 & $-0,334$ & 0,522 & 0,079 & $-0,142$ & 0,099 & 0,170 \\
\hline Artenzahl Moose & & & 1,000 & 0,297 & 0,412 & 0,340 & 0,148 & $-0,152$ & 0,377 \\
\hline Artenzahl Flechten & & & & 1,000 & $-0,112$ & $-0,027$ & 0,156 & $-0,216$ & $-0,029$ \\
\hline Humusgehalt [\%] & & & & & 1,000 & 0,216 & $-0,065$ & 0,014 & 0,410 \\
\hline $\mathrm{CaCO}_{3}$ (ordinal) & & & & & & 1,000 & 0,610 & $-0,420$ & 0,852 \\
\hline pH-Wert & & & & & & & 1,000 & $-0,440$ & 0,583 \\
\hline H-Wert [mval/100 g] & & & & & & & & 1,000 & $-0,342$ \\
\hline \multirow[t]{2}{*}{ S-Wert [mval/100 g] } & & & & & & & & & 1,000 \\
\hline & $\begin{array}{c}\text { Basen- } \\
\text { sättigung } \\
{[\%]}\end{array}$ & $\begin{array}{c}\text { Kationen- } \\
\text { austausch- } \\
\text { kapazität } \\
{[\mathrm{mval} / 100 \mathrm{~g}]}\end{array}$ & $\begin{array}{c}\text { Tief- } \\
\text { gründig- } \\
\text { keit } \\
\text { (ordinal) }\end{array}$ & $\begin{array}{l}\text { Höhe ü. } \\
\text { NN [m] }\end{array}$ & $\begin{array}{c}\text { Mikro- } \\
\text { relief } \\
{[\mathrm{cm}]}\end{array}$ & $\begin{array}{c}\text { Über- } \\
\text { stauung } \\
{[+ \text { oder - }]}\end{array}$ & $\begin{array}{c}\text { Gesteins- } \\
\text { anteil } \\
\text { (ordinal) }\end{array}$ & $\begin{array}{c}\text { Deckung } \\
\text { Gestein [\%] }\end{array}$ & $\begin{array}{c}\text { Größe } \\
\text { Trockenrasen- } \\
\text { gebiete } \\
\text { (ordinal) }\end{array}$ \\
\hline Gesamtartenzahl & 0,055 & 0,254 & $-0,461$ & 0,148 & 0,359 & 0,336 & 0,284 & 0,318 & $-0,205$ \\
\hline \begin{tabular}{|l} 
Artenzahl \\
Gefäßpflanzen \\
\end{tabular} & $-0,099$ & 0,174 & $-0,263$ & 0,229 & 0,210 & 0,053 & 0,030 & 0,047 & $-0,495$ \\
\hline Artenzahl Moose & 0,148 & 0,374 & $-0,408$ & 0,081 & 0,389 & 0,508 & 0,351 & 0,372 & 0,059 \\
\hline Artenzahl Flechten & 0,216 & $-0,038$ & $-0,169$ & $-0,155$ & 0,150 & 0,264 & 0,311 & 0,350 & 0,449 \\
\hline Humusgehalt [\%] & $-0,010$ & 0,418 & $-0,640$ & 0,191 & 0,137 & 0,309 & 0,303 & 0,292 & $-0,165$ \\
\hline $\mathrm{CaCO}_{3}$ (ordinal) & 0,421 & 0,845 & $-0,532$ & 0,007 & 0,161 & 0,250 & 0,505 & 0,429 & 0,074 \\
\hline pH-Wert & 0,441 & 0,573 & $-0,237$ & $-0,047$ & 0,086 & 0,161 & 0,427 & 0,421 & 0,264 \\
\hline H-Wert $[\mathrm{mval} / 100 \mathrm{~g}]$ & $-1,000$ & $-0,313$ & 0,141 & 0,100 & $-0,112$ & $-0,148$ & $-0,243$ & $-0,223$ & $-0,239$ \\
\hline S-Wert [mval/100 g] & 0,344 & 0,999 & $-0,629$ & 0,022 & 0,154 & 0,208 & 0,622 & 0,503 & $-0,004$ \\
\hline Basensättigung [\%] & 1,000 & 0,315 & $-0,143$ & $-0,098$ & 0,108 & 0,148 & 0,244 & 0,223 & 0,236 \\
\hline \begin{tabular}{|l|} 
Kationenaustausch- \\
kapazität [mval/100 g] \\
\end{tabular} & & 1,000 & $-0,633$ & 0,028 & 0,150 & 0,205 & 0,619 & 0,503 & $-0,011$ \\
\hline $\begin{array}{l}\text { Tiefgründigkeit } \\
\text { (ordinal) }\end{array}$ & & & 1,000 & 0,012 & $-0,097$ & $-0,379$ & $-0,705$ & $-0,631$ & $-0,022$ \\
\hline Höhe ü. NN [m] & & & & 1,000 & 0,007 & 0,054 & $-0,019$ & $-0,011$ & $-0,197$ \\
\hline Mikrorelief [cm] & & & & & 1,000 & 0,231 & 0,059 & 0,047 & $-0,050$ \\
\hline Überstauung [+ oder -] & & & & & & 1,000 & 0,259 & 0,313 & 0,173 \\
\hline Gesteinsanteil (ordinal) & & & & & & & 1,000 & 0,787 & 0,103 \\
\hline Deckung Gestein [\%] & & & & & & & & 1,000 & 0,102 \\
\hline $\begin{array}{l}\text { Größe Trockenrasen- } \\
\text { gebiete (ordinal) }\end{array}$ & & & & & & & & & 1,000 \\
\hline
\end{tabular}

\subsubsection{Bodenparameter}

Die Trockenrasen Saaremaas zeigen auf Hauptgruppen- sowie Assoziationsebene standörtliche Unterschiede bezogen auf die untersuchten Bodenparameter. Im Folgenden werden die Hauptgruppen sowie die unterschiedlichen Trockenrasenassoziationen hinsichtlich ihrer bodenökologischen Standortparameter verglichen. In Tab. 20 wird ein Überblick der untersuchten Bodenparameter mit arithmetischem Mittel, Median, Minimum und Maximum sowie Standardabweichung bezogen auf sämtliche Aufnahmen gegeben. Die Differenzen der Untereinheiten wurden bereits in Abschnitt 4.1 erläutert. In Tab. 21 wird ein Überblick der untersuchten Bodenparameter in den Trockenrasenassoziationen einschließlich deren Untereinheiten gegeben. In Tab. 22 werden die Bodenparameter der vorkommenden Haupt- 
gruppen und in Tab. 23 der Trockenrasenassoziationen durch einen Post-hoc-Mehfachvergleich gruppiert.

Tab. 20: Übersicht der untersuchten Bodenparameter bezogen auf sämtliche Aufnahmen $(n=$ Anzahl der Aufnahmen, $\varnothing=$ arithmetisches Mittel, $\mathrm{s}=$ Standardabweichung).

\begin{tabular}{|c|c|c|c|c|c|c|c|c|c|c|}
\hline$n=231$ & $\begin{array}{l}\text { Tief- } \\
\text { gründig- } \\
\text { keit } \\
\text { (ordinal) }\end{array}$ & $\begin{array}{l}\text { Gesteins- } \\
\text { anteil } \\
\text { (ordinal) }\end{array}$ & $\begin{array}{c}\mathrm{CaCO}_{3} \\
\text { (ordinal) }\end{array}$ & $\begin{array}{c}\text { Humus- } \\
\text { gehalt [\%] }\end{array}$ & $\begin{array}{c}\mathrm{pH}-\text { Wert } \\
{\left[\mathrm{H}_{2} \mathrm{O}\right]}\end{array}$ & $\begin{array}{c}\text { H-Wert } \\
{[\mathrm{mval} / 100 \mathrm{~g}]}\end{array}$ & $\begin{array}{c}\text { S-Wert } \\
{[\mathrm{mval} / 100 \mathrm{~g}]}\end{array}$ & $\begin{array}{c}\text { Kationen- } \\
\text { austausch- } \\
\text { kapazität } \\
\text { [mval/100 g] }\end{array}$ & $\begin{array}{c}\text { Basen- } \\
\text { sättigung } \\
{[\%]}\end{array}$ & $\begin{array}{l}\text { Mikro- } \\
\text { relief [cm] }\end{array}$ \\
\hline$\varnothing$ & 3,1 & 2,3 & 2,6 & 7,4 & 7,3 & 0,1 & 40,1 & 40,3 & 99,2 & 4,6 \\
\hline Median & 3 & 1 & 3 & 5,5 & 7,5 & 0 & 38,4 & 39 & 100 & 3 \\
\hline $\mathrm{s}$ & 1,5 & 1,7 & 1,5 & 9,3 & 0,7 & 1,1 & 26,7 & 26,6 & 3,6 & 4,2 \\
\hline Minimum & 1,0 & 1,0 & 0,0 & 0,0 & 4,6 & 0,0 & 1,2 & 1,2 & 67,2 & 1,0 \\
\hline Maximum & 5,0 & 6,0 & 5,0 & 60,6 & 8,2 & 13,7 & 99,0 & 99,0 & 100,0 & 30,0 \\
\hline
\end{tabular}

Tab. 21: Übersicht der untersuchten Bodenparameter getrennt nach den vorkommenden Trockenrasentypen und deren Untereinheiten (vgl. Abschnitt 4.1) $(n=$ Anzahl der Aufnahmen, Med = Median, $\varnothing=$ Arithmetisches Mittel, s = Standardabweichung).

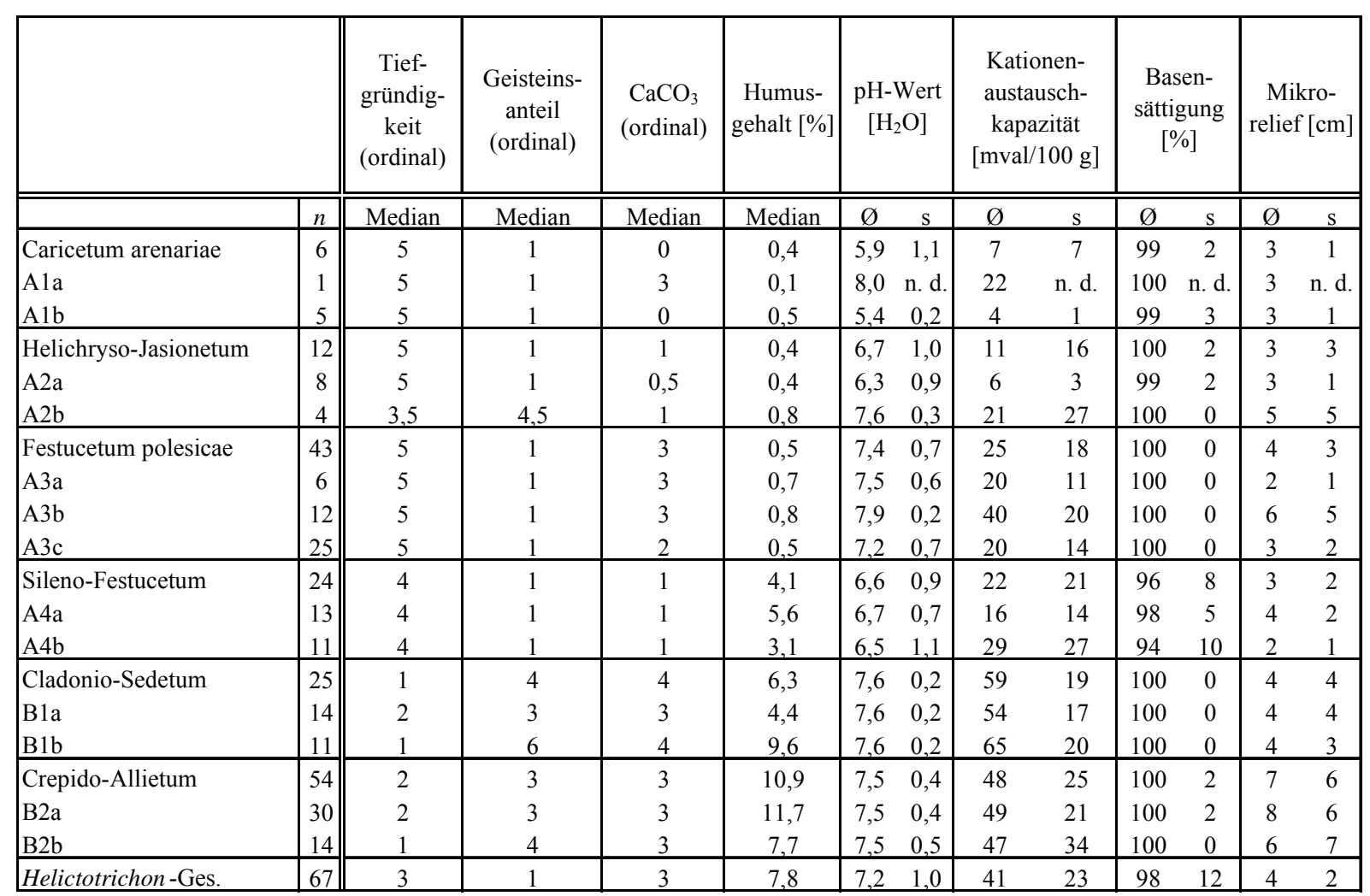


Tab. 22: Ergebnisse der Post-hoc-Mehrfachvergleiche (Tukey-HSD) wichtiger Bodenparameter der Trockenrasen auf Saaremaa getrennt nach Hauptgruppen. Übereinstimmende Buchstaben kennzeichnen jeweils homogene Gruppen bezüglich des jeweiligen Parameters auf Basis eines 95\%-Konfidenzintervalles $(n=$ Anzahl der Aufnahmen).

\begin{tabular}{|l|c||c|c|c|c|c|c|c|}
\hline$n$ & $\begin{array}{c}\text { Tief- } \\
\text { gründigkeit } \\
\text { (ordinal) }\end{array}$ & $\begin{array}{c}\text { Geisteins- } \\
\text { anteil } \\
\text { (ordinal) }\end{array}$ & $\begin{array}{c}\mathrm{CaCO}_{3} \\
\text { (ordinal) }\end{array}$ & $\begin{array}{c}\mathrm{pH} \text {-Wert } \\
{\left[\mathrm{H}_{2} \mathrm{O}\right]}\end{array}$ & $\begin{array}{c}\text { Basen- } \\
\text { sättigung } \\
{[\%]}\end{array}$ & $\begin{array}{c}\text { Kationen- } \\
\text { austausch- } \\
\text { kapazität } \\
{[\mathrm{mval} / 100 \mathrm{~g}]}\end{array}$ & $\begin{array}{c}\text { Mikro- } \\
\text { relief } \\
{[\mathrm{cm}]}\end{array}$ \\
\hline \hline Koelerio-Corynephorenea & 85 & $\mathrm{c}$ & $\mathrm{a}$ & $\mathrm{a}$ & $\mathrm{a}$ & $\mathrm{a}$ & $\mathrm{a}$ & $\mathrm{a}$ \\
\hline Sedo-Scleranthenea & 79 & $\mathrm{a}$ & $\mathrm{b}$ & $\mathrm{b}$ & $\mathrm{b}$ & $\mathrm{a}$ & $\mathrm{c}$ & $\mathrm{b}$ \\
\hline Festuco-Brometea & 67 & $\mathrm{~b}$ & $\mathrm{a}$ & $\mathrm{a}$ & $\mathrm{a}$ & $\mathrm{a}$ & $\mathrm{b}$ & $\mathrm{a}$ \\
\hline
\end{tabular}

Tab. 23: Ergebnisse der Post-hoc-Mehrfachvergleiche (Tukey-HSD) wichtiger Bodenparameter in den unterschiedlichen Trockenrasentypen auf Saaremaa. Übereinstimmende Buchstaben kennzeichnen jeweils homogene Gruppen bezüglich des jeweiligen Parameters auf Basis eines 95 \%-Konfidenzintervalles $(n=$ Anzahl der Aufnahmen).

\begin{tabular}{|l|c||c|c|c|c|c|c|c|}
\hline & $n$ & $\begin{array}{c}\text { Tief- } \\
\text { gründigkeit } \\
\text { (ordinal) }\end{array}$ & $\begin{array}{c}\text { Geisteins- } \\
\text { anteil } \\
\text { (ordinal) }\end{array}$ & $\begin{array}{c}\mathrm{CaCO}_{3} \\
\text { (ordinal) }\end{array}$ & $\begin{array}{c}\mathrm{pH}-\text { Wert } \\
\left(\mathrm{H}_{2} \mathrm{O}\right)\end{array}$ & $\begin{array}{c}\text { Basen- } \\
\text { sättigung } \\
{[\%]}\end{array}$ & $\begin{array}{c}\text { Kationen- } \\
\text { austausch- } \\
\text { kapazität } \\
{[\mathrm{mval} / 100 \mathrm{~g}]}\end{array}$ & $\begin{array}{c}\text { Mikro- } \\
\text { relief [cm] }\end{array}$ \\
\hline \hline Caricetum arenariae & 6 & $\mathrm{~d}, \mathrm{c}$ & $\mathrm{a}$ & $\mathrm{a}$ & $\mathrm{a}$ & $\mathrm{a}, \mathrm{b}$ & $\mathrm{a}$ & $\mathrm{a}, \mathrm{b}$ \\
\hline Helichryso-Jasionetum & 12 & $\mathrm{~d}, \mathrm{c}$ & $\mathrm{a}$ & $\mathrm{a}$ & $\mathrm{b}, \mathrm{c}$ & $\mathrm{b}$ & $\mathrm{a}$ & $\mathrm{a}$ \\
\hline Festucetum polesicae & 43 & $\mathrm{~d}, \mathrm{c}$ & $\mathrm{a}$ & $\mathrm{b}, \mathrm{c}$ & $\mathrm{d}$ & $\mathrm{b}$ & $\mathrm{a}, \mathrm{b}$ & $\mathrm{a}, \mathrm{b}$ \\
\hline Sileno-Festucetum & 24 & $\mathrm{c}$ & $\mathrm{a}$ & $\mathrm{a}, \mathrm{b}$ & $\mathrm{b}$ & $\mathrm{a}$ & $\mathrm{a}, \mathrm{b}$ & $\mathrm{a}$ \\
\hline Cladonio-Sedetum & 25 & $\mathrm{a}$ & $\mathrm{b}$ & $\mathrm{d}$ & $\mathrm{d}$ & $\mathrm{b}$ & $\mathrm{c}$ & $\mathrm{a}, \mathrm{b}$ \\
\hline Crepido-Allietum & 54 & $\mathrm{a}$ & $\mathrm{b}$ & $\mathrm{c}, \mathrm{d}$ & $\mathrm{d}$ & $\mathrm{b}$ & $\mathrm{c}$ & $\mathrm{b}$ \\
\hline Helictotrichon -Ges. & 67 & $\mathrm{~b}$ & $\mathrm{a}$ & $\mathrm{c}, \mathrm{d}$ & $\mathrm{c}, \mathrm{d}$ & $\mathrm{b}$ & $\mathrm{b}, \mathrm{c}$ & $\mathrm{a}, \mathrm{b}$ \\
\hline
\end{tabular}

\section{Tiefgründigkeit des Bodens}

Die Tiefgründigkeitsklassen der Böden in den Trockenrasen auf Saaremaa reichen von 1 $(=0-5 \mathrm{~cm})$ bis $5(>40 \mathrm{~cm})$ (Tab. 20). Auf Hauptgruppenebene ergab sich eine eindeutige Trennung (Tab. 22). Die Gesellschaften der Koelerio-Corynephorenea besiedeln größtenteils Böden mit einer mittleren Tiefgründigkeitsklasse von $5(>40 \mathrm{~cm})$ und somit die tiefgründigsten Böden. Eine Ausnahme stellen die Böden des Sileno-Festucetum mit mittlerer Tiefgründigkeitsklasse $4(=25-40 \mathrm{~cm})$ dar. Sie sind somit hinsichtlich der mittleren Bodenmächtigkeit zwischen den Beständen der Hauptgruppen Koelerio-Corynephorenea und der Festuco-Brometea (Tief-gründigkeitsklasse 3, $=10-25 \mathrm{~cm}$ ) anzusiedeln. Die Gesellschaften der Sedo-Scleranthenea besiedeln die flachgründigsten Böden, wobei das Crepido-Allietum vorwiegend auf Böden mit einer Tiefgründigkeitsklasse $2(=5-10 \mathrm{~cm})$ und somit auf etwas tiefgründigeren Böden als das Cladonio-Sedetum wächst (Tab. 21).

\section{Gesteinsanteil des Bodens}

Die Böden der Trockenrasen auf Saaremaa weisen im Mittel eine Geisteinsanteilklasse von 1 $(<1 \%)$ auf. Die Spanne reicht jedoch von 1 bis zu einem Maximalwert von $6(>75 \%)$ bei einigen Aufnahmen (Tab. 20). Bei den Sand- und Halbtrockenrasen liegt der Gesteinsanteil der Böden größtenteils bei Klasse 1, im Falle des Helichryso-Jasionetum teilweise auch etwas höher (vgl. Abschnitt 4.1.2). Das Crepido-Allietum kommt auf mittel steinigen (Klasse 3, $=10-30 \%$ ) und das Cladonio-Sedetum auf stark steinigen Böden (Klasse 4, $=30-50 \%$ ) vor 
(Tab. 21). Der Post-hoc-Mehrfachvergleich ergibt eine klare Trennung der SedoScleranthenea-Gesellschaften von den übrigen Trockenrasen (Tab. 23, Tab. 22).

\section{Carbonatgehalt}

Der durchschnittliche Carbonatgehalt des Bodens liegt insgesamt mit Gehaltsklasse 3 (=2$10 \%$ ) relativ hoch. Die Spanne in den Aufnahmen reicht insgesamt von 0 (kalkfrei) bis 5 (>25\%) (Tab. 20). Die Felsgrusgesellschaften kommen im Durchschnitt auf etwas carbonatreicheren Böden vor als die Sandtrockenrasen und die Helictotrichon-Gesellschaft (Tab. 22). Auf Assoziationsebene ergibt sich in den meisten Fällen keine eindeutige Trennung. (Tab. 23). Das Caricetum arenariae und das Helichryso-Jasionetum besiedeln die am stärksten entkalkten (Carbonatgehaltsklasse 1, (>1\%), das Cladonio-Sedetum die carbonatreichsten Böden (Carbonatgehaltsklasse 4, $=10-25 \%$ ). Das Festucetum polesicae wächst auf küstennahen Dünen mit einem hohen Anteil an Muschelkalk, weshalb der Carbonatgehalt mit mittlerer Gehaltsklasse 3 höher liegt als jener der Böden in den übrigen Sandtrockenrasen (Tab. 21).

\section{Humusgehalt}

Der mittlere Humusgehalt des Oberbodens liegt in den untersuchten Beständen bei 5,5\% (Median). Hervorzuheben ist der Maximalwert von 60,6\% in Aufnahme X01 (SilenoFestucetum; gemähte Wiese bei der das Mahdgut nicht entfernt wird) (Tab. 20). Die Böden der meisten Sandtrockenrasengesellschaften sind sehr humusarm $(<1 \%)$. Eine Ausnahme stellt das Sileno-Festucetum dar. Der Humusanteil liegt hier mit einem Median von 4,1\% deutlich über den restlichen Koelerio-Corynephorenea-Gesellschaften, jedoch etwas niedriger als bei den Böden der Helictotrichon-Gesellschaft. Ein bemerkenswerter Unterschied ist bei den Gesellschaften der Felsgrusfluren festzustellen. Die schwarzen Böden des CrepidoAllietum haben einen Humusgehalt von 10,9\% (Median), während jene des CladonioSedetum mit 6,3\% (Median) deutlich humusärmer sind (Tab. 21).

\section{pH-Wert}

Insgesamt ist der $\mathrm{pH}-$ Wert im Oberboden der Trockenrasen über dem neutralen Bereich. Der niedrigste Wert wurde in Aufnahme L14 (Sileno-Festucetum) mit 4,6 gemessen. Die pHWerte der Böden einiger Bestände auf marinen Sanden liegen oberhalb von 8. Das Maximum liegt bei 8,2 (Tab. 20). Bezogen auf die Hauptgruppen bilden die Felsgrusgesellschaften eine abgetrennte homogene Gruppe mit etwas höheren pH-Werten (Tab. 22). Das Caricetum arenariae ist deutlich von den übrigen Trockenrasenassoziationen getrennt (Tab. 23). Es kommt auf leicht sauren Böden mit einem mittleren $\mathrm{pH}-$ Wert von 5,9 vor. Die mittleren $\mathrm{pH}-$ Werte der Böden des Festucetum polesicae, des Crepido-Allietum und des Cladonio-Sedetum sind am höchsten (Tab. 23). Sie liegen, ebenso wie bei der Helictotrichon-Gesellschaft, oberhalb des Neutralpunktes. Die Unterschiede in den verschiedenen Gesellschaften sind jedoch relativ gering (Tab. 21).

\section{Kationenaustauschkapazität und Basensättigung}

Die Spanne der Kationenaustauschkapazität der untersuchten Böden reicht von 1,2 (J05, Festucetum polesicae) bis 99,0 (K04, Crepido-Allietum) (Tab. 20). Auf Hauptgruppenebene lassen sich drei homogene Gruppen unterscheiden. Die Kationenaustauschkapazität ist bei den Böden der Unterklasse Sedo-Scleranthenea am höchsten, gefolgt von den FestucoBrometea und am niedrigsten in den Koelerio-Corynephorenea (Tab. 21, Tab. 23). 
Die untersuchten Böden haben im Allgemeinen eine sehr gute Basenversorgung mit einer Basensättigung von nahezu $100 \%$ (Tab. 20). Auf Hauptgruppenebene ergab der Post-hocMehrfachvergleich keine Trennung in homogene Gruppen (Tab. 22). Die Assoziationen lassen sich jedoch in zwei homogene Gruppen unterscheiden. Das Sileno-Festucetum ist dabei mit den niedrigsten Werten von den übrigen Gesellschaften getrennt (Tab. 23).

\section{Mikrorelief}

Die Spanne des gemessenen Mikroreliefs reicht insgesamt von 1 bis $30 \mathrm{~cm}$. Der Maximalwert wurde bei Aufnahme V02 (Crepido-Allietum; Bestand von einer Steilküste im Westen der Insel Vilsandi) gemessen (Tab. 20). Die Bestände des Crepido-Allietum bilden bezüglich des Mikroreliefs eine deutlich von den übrigen Assoziationen getrennte homogene Gruppe (Tab. 23). Das ausgeprägte Mikrorelief von durchschnittlich $7 \mathrm{~cm}$ ist ein charakteristisches Merkmal der Böden dieser Gesellschaft. Bei den restlichen Gesellschaften sind kaum nennenswerte Unterschiede erkennbar (Tab. 21).

\section{Korrelationen der Bodenparameter}

Im Folgenden werden die wichtigsten Korrelationen zwischen den Bodenparametern mit $p>0,6$, welche in Tab. 19 aufgeführt sind herausgegriffen.

Ein niedriger Humusgehalt geht einher mit tiefgründigen Böden. Der Carbonatgehalt ist stark mit dem pH- und S-Wert sowie der Kationenaustauschkapazität korreliert. Der H-Wert und die Basensättigung sind co-korreliert. Außerdem beeinflussen sich der S-Wert, die Kationenaustauschkapazität, die Tiefgründigkeit und der Gesteinsanteil. Zwischen den beiden letztgenannten Parametern und der Deckung der Gesteine besteht ebenso ein Zusammenhang.

\subsubsection{Deckungsgrade der Vegetation und andere Strukturparameter}

Tab. 24 gibt einen Überblick über die Vegetationsdeckung sowie über wichtige Strukturparameter mit arithmetischem Mittel, Median, Minimum, Maximum und Standardabweichung bezogen auf sämtliche Aufnahmen. In Tab. 25 sind die mittleren Deckungsgrade der Vegetation sowie wichtige Strukturparameter getrennt nach den vorkommenden Trockenrasengesellschaften und ihren Untereinheiten dargestellt. Die auffälligsten Kennwerte werden im Folgenden herausgegriffen und verglichen. Des Weiteren werden hochsignifikante Zusammenhänge angegeben, die anhand von bivariaten Korrelationsanalysen ermittelt wurden $(n=167)$. Die Post-hoc-Mehrfachvergleiche verdeutlichen die Unterschiede in der Vegeta-tionsdeckung sowie die Unterschiede der Strukturparameter auf Hauptgruppen(Tab. 26) und Assoziationsebene (Tab. 27).

Tab. 24: Übersicht der prozentualen Deckungsgrade der Vegetation sowie wichtiger Strukturparameter bezogen auf sämtliche Aufnahmen $(n=231)(\varnothing=$ arithmetisches Mittel, $s=$ Standardabweichung).

\begin{tabular}{|l||c|c|c|c|c|c|c|}
\hline Deckung [\%] ... & $\begin{array}{c}\text { Vegetation } \\
\text { gesamt }\end{array}$ & $\begin{array}{c}\text { Gefäß- } \\
\text { pflanzen }\end{array}$ & Moose & Flechten & Gestein & $\begin{array}{c}\text { Krypto- } \\
\text { gamen- } \\
\text { bewuchs der } \\
\text { Gesteine }\end{array}$ & Streu \\
\hline \hline$\varnothing$ & 80 & 59 & 52 & 15 & 9 & 29 & 17 \\
\hline Median & 90 & 60 & 55 & 5 & 1 & 20 & 10 \\
\hline s & 22 & 26 & 27 & 19 & 18 & 27 & 16 \\
\hline Minimum & 4 & 4 & 0 & 0 & 0 & 0 & 1 \\
\hline Maximum & 100 & 100 & 98 & 95 & 93 & 95 & 60 \\
\hline
\end{tabular}


Tab. 25: Übersicht der mittleren prozentualen Deckungsgrade der Vegetation sowie wichtiger Strukturparameter getrennt nach den vorkommenden Trockenrasengesellschaften und ihren Untereinheiten $(n=$ Anzahl der Aufnahmen, $\varnothing=$ arithmetisches Mittel, $\mathrm{s}=$ Standardabweichung).

\begin{tabular}{|c|c|c|c|c|c|c|c|c|c|c|c|c|c|}
\hline \multicolumn{2}{|l|}{ Deckung [\%] } & \multicolumn{2}{|c|}{$\begin{array}{l}\text { Vegetation } \\
\text { gesamt }\end{array}$} & \multicolumn{2}{|c|}{$\begin{array}{l}\text { Gefäß- } \\
\text { pflanzen }\end{array}$} & \multicolumn{2}{|c|}{ Moose } & \multicolumn{2}{|c|}{ Flechten } & \multirow{2}{*}{$\begin{array}{l}\text { Gestein } \\
\text { Median }\end{array}$} & \multirow{2}{*}{$\begin{array}{c}\text { Krypto- } \\
\text { gamen- } \\
\text { bewuchs } \\
\text { der } \\
\text { Gesteine } \\
\text { Median }\end{array}$} & \multicolumn{2}{|c|}{ Streu } \\
\hline & $n$ & $\varnothing$ & $\mathrm{s}$ & $\varnothing$ & $\mathrm{s}$ & $\varnothing$ & $\mathrm{s}$ & $\varnothing$ & $\mathrm{s}$ & & & $\varnothing$ & $\mathrm{s}$ \\
\hline Caricetum arenariae & 6 & 68 & 38 & 30 & 15 & 30 & 20 & 48 & 32 & 0 & 0 & 15 & 10 \\
\hline Ala & 1 & 4 & 0 & 4 & 0 & 0 & 0 & 0 & 0 & 0 & 0 & 1 & 0 \\
\hline $\mathrm{A} 1 \mathrm{~b}$ & 5 & 81 & 23 & 35 & 10 & 36 & 15 & 57 & 24 & 0 & 0 & 18 & 8 \\
\hline Helichryso-Jasionetum & 12 & 66 & 25 & 30 & 11 & 32 & 23 & 31 & 26 & 0 & 15 & 9 & 8 \\
\hline A. $2 \mathrm{a}$ & 8 & 77 & 24 & 32 & 10 & 39 & 24 & 41 & 25 & 0 & 0 & 11 & 9 \\
\hline $\mathrm{A} 2 \mathrm{~b}$ & 4 & 45 & 7 & 25 & 12 & 19 & 15 & 10 & 14 & 27,5 & 15 & 5 & 4 \\
\hline Festucetum polesicae & 43 & 72 & 22 & 37 & 16 & 47 & 25 & 23 & 16 & 0 & 0 & 12 & 8 \\
\hline A3a & 6 & 48 & 27 & 31 & 20 & 18 & 16 & 12 & 14 & 0 & 0 & 7 & 5 \\
\hline $\mathrm{A} 3 \mathrm{~b}$ & 12 & 81 & 13 & 46 & 12 & 50 & 18 & 27 & 19 & 0 & 5 & 14 & 10 \\
\hline $\mathrm{A} 3 \mathrm{c}$ & 25 & 73 & 21 & 35 & 16 & 53 & 26 & 24 & 14 & 0 & 0 & 12 & 8 \\
\hline Sileno-Festucetum & 24 & 89 & 17 & 65 & 24 & 66 & 25 & 13 & 14 & 0 & 0 & 18 & 12 \\
\hline $\mathrm{A} 4 \mathrm{a}$ & 13 & 97 & 6 & 68 & 26 & 80 & 11 & 21 & 14 & 0 & 0 & 18 & 11 \\
\hline $\mathrm{A} 4 \mathrm{~b}$ & 11 & 80 & 21 & 62 & 21 & 49 & 26 & 4 & 8 & 1 & 0 & 17 & 13 \\
\hline Cladonio-Sedetum & 25 & 71 & 28 & 51 & 22 & 49 & 27 & 10 & 17 & 10 & 25 & 12 & 12 \\
\hline B1a & 14 & 87 & 11 & 57 & 18 & 59 & 21 & 16 & 21 & 2 & 20 & 13 & 12 \\
\hline B1b & 11 & 51 & 30 & 45 & 26 & 35 & 28 & 2 & 3 & 40 & 40 & 10 & 12 \\
\hline Crepido-Allietum & 54 & 77 & 19 & 56 & 20 & 43 & 24 & 19 & 20 & 10 & 20 & 8 & 6 \\
\hline B2a & 30 & 54 & 40 & 34 & 35 & 29 & 27 & 10 & 17 & 8 & 17,5 & 8 & 6 \\
\hline $\mathrm{B} 2 \mathrm{~b}$ & 14 & 42 & 37 & 9 & 11 & 13 & 22 & 14 & 21 & 17,5 & 40 & 5 & 3 \\
\hline Helictotrichon-Ges. & 67 & 92 & 12 & 83 & 17 & 65 & 24 & 3 & 7 & 0 & 25 & 31 & 19 \\
\hline
\end{tabular}

Tab. 26: Ergebnisse der Post-hoc-Mehrfachvergleiche (Tukey-HSD) für die Deckungsgrade der Vegetation und für wichtige Strukturparameter in den unterschiedlichen Trockenrasen auf Saaremaa getrennt nach Hauptgruppen. Übereinstimmende Buchstaben kennzeichnen jeweils homogene Gruppen bezüglich der Vegetationsdeckung sowie der Strukturparameter auf Basis eines 95\%-Konfidenzintervalles $(n=$ Anzahl der Aufnahmen).

\begin{tabular}{|l|c||c|c|c|c|c|c|}
\hline Deckung [\%] ... & $n$ & $\begin{array}{c}\text { Vegetation } \\
\text { gesamt }\end{array}$ & $\begin{array}{c}\text { Gefäß- } \\
\text { pflanzen }\end{array}$ & Moose & Flechten & Gestein & Streu \\
\hline \hline Koelerio-Corynephorenea & 85 & $\mathrm{a}$ & $\mathrm{a}$ & $\mathrm{a}$ & $\mathrm{c}$ & $\mathrm{a}$ & $\mathrm{a}$ \\
\hline Sedo-Scleranthenea & 79 & $\mathrm{a}$ & $\mathrm{b}$ & $\mathrm{a}$ & $\mathrm{b}$ & $\mathrm{b}$ & $\mathrm{a}$ \\
\hline Festuco-Brometea & 67 & $\mathrm{~b}$ & $\mathrm{c}$ & $\mathrm{b}$ & $\mathrm{a}$ & $\mathrm{a}$ & $\mathrm{b}$ \\
\hline
\end{tabular}


Tab. 27: Ergebnisse der Post-hoc-Mehrfachvergleiche (Tukey-HSD) für die Deckungsgrade der Vegetation und für wichtige Strukturparameter in den unterschiedlichen Trockenrasentypen auf Saaremaa. Übereinstimmende Buchstaben kennzeichnen jeweils homogene Gruppen bezüglich der Vegetationsdeckung sowie der Strukturparameter auf Basis eines 95 \%-Konfidenzintervalles ( $n=$ Anzahl der Aufnahmen).

\begin{tabular}{|l|c||c|c|c|c|c|c|}
\hline Deckung [\%] ... & $n$ & $\begin{array}{c}\text { Vegetation } \\
\text { gesamt }\end{array}$ & $\begin{array}{c}\text { Gefäß- } \\
\text { pflanzen }\end{array}$ & Moose & Flechten & Gestein & Streu \\
\hline \hline Caricetum arenariae & 6 & $\mathrm{a}$ & $\mathrm{a}$ & $\mathrm{a}$ & $\mathrm{d}$ & $\mathrm{a}$ & $\mathrm{a}$ \\
\hline Helichryso-Jasionetum & 12 & $\mathrm{a}$ & $\mathrm{a}$ & $\mathrm{a}$ & $\mathrm{c}$ & $\mathrm{a}, \mathrm{b}$ & $\mathrm{a}$ \\
\hline Festucetum polesicae & 43 & $\mathrm{a}, \mathrm{b}$ & $\mathrm{a}, \mathrm{b}$ & $\mathrm{a}, \mathrm{b}$ & $\mathrm{b}, \mathrm{c}$ & $\mathrm{a}$ & $\mathrm{a}$ \\
\hline Sileno-Festucetum & 24 & $\mathrm{~b}, \mathrm{c}$ & $\mathrm{c}, \mathrm{d}$ & $\mathrm{b}$ & $\mathrm{a}, \mathrm{b}$ & $\mathrm{a}$ & $\mathrm{a}$ \\
\hline Cladonio-Sedetum & 25 & $\mathrm{a}, \mathrm{b}$ & $\mathrm{b}, \mathrm{c}$ & $\mathrm{a}, \mathrm{b}$ & $\mathrm{a}, \mathrm{b}$ & $\mathrm{b}$ & $\mathrm{a}$ \\
\hline Crepido-Allietum & 54 & $\mathrm{a}, \mathrm{b}, \mathrm{c}$ & $\mathrm{c}$ & $\mathrm{a}, \mathrm{b}$ & $\mathrm{a}, \mathrm{b}, \mathrm{c}$ & $\mathrm{a}, \mathrm{b}$ & $\mathrm{a}$ \\
\hline Helictotrichon -Ges. & 67 & $\mathrm{c}$ & $\mathrm{d}$ & $\mathrm{b}$ & $\mathrm{a}$ & $\mathrm{a}$ & $\mathrm{b}$ \\
\hline
\end{tabular}

Insgesamt reicht die Spanne der Gesamtdeckung der Vegetation von $4 \%$ (Ö05, Caricetum arenariae) bis $100 \%$ (Tab. 24). Die Bestände der Klasse Festuco-Brometea weisen die höchsten mittleren Gesamtdeckungsgrade auf und bilden eine klar von den beiden anderen Hauptgruppen getrennte homogene Gruppe (Tab. 26). Auch auf Assoziationsebene liegen die dichten Bestände der Helictotrichon-Gesellschaft deutlich getrennt am oberen Ende der Skala. Vergleichbar dichte Bestände bildet die Vegetation des Sileno-Festucetum aus. Die geringsten Deckungsgrade werden im Helichryso-Jasionetum und im Caricetum arenariae erreicht. In den übrigen Gesellschaften schwanken die Werte teilweise sehr stark, weshalb eine eindeutige Trennung in homogene Gruppen nicht möglich ist (Tab. 27).

Die Gefäßpflanzen haben insgesamt eine mittlere Deckung von etwa $60 \%$ (Tab. 24). Auf Hauptgruppenebene lässt sich eine eindeutige Trennung nachvollziehen. Die niedrigsten Deckungswerte kommen in den Koelerio-Corynephorenea, die höchsten in den FestucoBrometea vor (Tab. 26). Klar getrennt von den restlichen Trockenrasenassoziationen sind am unteren Ende der Skala die auf Dünen wachsenden Gesellschaften Helichryso-Jasionetum und Caricetum arenariae sowie am oberen Ende die Helictotrichon-Gesellschaft (Tab. 27). Bei letztgenannter ist der besonders hohe mittlere Wert von $83 \%$ hervorzuheben (Tab. 25).

Die Spanne der Moosdeckung reicht in den Trockenrasen auf Saaremaa von $0 \%$ bis $98 \%$. Das arithmetische Mittel liegt nur geringfügig unterhalb des Wertes der Gefäßpflanzendeckung (Tab. 24). Auf Hauptgruppenebene lassen sich zwei homogene Gruppen unterscheiden. Die dichtesten Bestände bilden die Moose in der Klasse Festuco-Brometea aus (Tab. 26). Bezogen auf die Assoziationen liegen das Helichryso-Jasionetum und das Caricetum arenariae deutlich am unteren, das Sileno-Festucetum und die HelictotrichonGesellschaft am oberen Ende der Skala (Tab. 27).

Die Verteilung der Werte der Flechtendeckung ist insgesamt sehr linksschief. Die Spanne reicht von $0 \%$ bis $95 \%$ bei einem Median von $5 \%$ (Tab. 24). Der Maximalwert wurde in Aufnahme X17 (Crepido-Allietum; Alvar bei Katri pank) dokumentiert. Die Hauptgruppen sind mit den geringsten Werten in den Festuco-Brometea, mittleren in den SedoScleranthenea und den höchsten in den Koelerio-Cornephorenea klar voneinander getrennt (Tab. 26). Eindeutig getrennt sind auf Assoziationsebene die Helictotrichon-Gesellschaft mit den geringsten, das Helichryso-Jasionetum mit mittleren, sowie das Caricetum arenariae mit den durchschnittlich höchsten Werten (Tab. 27).

Die Werte der Gesteinsdeckung sind ebenfalls linksschief verteilt (Tab. 24). Der höchste Wert von $93 \% \mathrm{kam}$ in Aufnahme X25 (Cladonio-Sedetum; fossiler Strandwall bei Pidula laht) vor. In den Koelerio-Corynephorenea sowie den Festuco-Brometea sind die Deckungsgrade offen liegender Gesteine äußerst gering. Die Bestände der Unterklasse Sedo-Scleranthenea bilden hingegen eine homogene Gruppe mit höheren Deckungsgraden (Tab. 26). 
Der Kryptogamenbewuchs der Gesteine weist eine weite Spanne auf. Der höchste Wert von $95 \%$ wurde auf einem Alvar westlich von Kuressaare festgestellt (N03, Crepido-Allietum). Die Gesteine in den Beständen der Helictotrichon-Gesellschaft weisen trotz einer deutlich geringeren mittleren Deckung den gleichen Grad an Kryptogamenbewuchs auf wie das Cladonio-Sedetum. Etwas geringere Werte sind im Crepido-Allietum zu verzeichnen. Die offen liegenden Gesteine in den Beständen der Sandtrockenrasen sind bis auf jene im Helichryso-Jasionetum frei von epilithisch wachsenden Kryptogamen (Tab. 25).

Die Spanne der Streudeckung reicht in den Trockenrasen auf Saaremaa von $1 \%$ bis $60 \%$ und beträgt im Durchschnitt $10 \%$ (Median) (Tab. 24). Die Halbtrockenrasen unterscheiden sich sowohl auf Hauptgruppen- als auch auf Assoziationsebene eindeutig von den übrigen Trockenrasen (Tab. 26, Tab. 27).

\section{Wechselbeziehungen}

Zwischen dem Deckungsgrad der Gefäßpflanzen und jenem der Moose besteht ein positiv linearer Zusammenhang $(r=0,442 ; p<0,001)$. Betrachtet man die Deckungsgrade der Moose, fällt auf, dass jene Gesellschaften mit einer geringen Deckung an Gefäßpflanzen tendenziell geringere Deckungsgrade an Moosen aufweisen als Gesellschaften mit einer hohen Deckung von Gefäßpflanzen (Tab. 25).

Der Deckungsgrad der Gefäßpflanzen ist signifikant negativ mit jenem der Flechten korreliert $(r=-0,318 ; p<0,001)$. Gesellschaften mit hohen Deckungsgraden an Gefäßpflanzen wie die Helictotrichon-Gesellschaft und das Sileno-Festucetum haben eine geringer ausgebildete Deckung der Flechtenschicht (Tab. 25).

Der Deckungsgrad der Streu ist positiv mit jenem der Gefäßpflanzen $(r=0,611 ; p<0,001)$ und der Moose $(r=0,329 ; p<0,001)$ sowie negativ mit jenem der Flechten $(r=-0,169$; $p=0,029)$ korreliert.

\subsubsection{Artenvielfalt}

Es sind deutliche Unterschiede bezüglich der mittleren Artenzahlen auf $4 \mathrm{~m}^{2}$ großen Flächen in den unterschiedlichen Trockenrasenassoziationen auf Saaremaa zu erkennen (Abb. 1).

Die Spanne der mittleren Artendichte auf $4 \mathrm{~m}^{2}$ ist in den unterschiedlichen auf Saaremaa vorkommenden Trockenrasengesellschaften relativ weit. Der niedrigste Wert wurde im Caricetum arenariae mit einer Art und der höchste im Crepido-Allietum mit 71 Arten festgestellt. Bezogen auf die Gefäßpflanzenartendichte liegt der Maximalwert bei 47 Arten auf $4 \mathrm{~m}^{2}$ (Z16; Helictotrichon-Gesellschaft). Der Durchschnitt beträgt 20,3 Arten. Hinsichtlich der Moosartendichte auf $4 \mathrm{~m}^{2}$ reicht die Spanne von 0 (Ö05, Caricetum arenariae; T11, Helichryso-Jasionetum; X02, Sileno-Festucetum) bis 27 Arten (T29, CrepidoAllietum). Auch die Flechtenartendichte variiert bezogen auf sämtliche Aufnahmen sehr stark. Es kommen Werte von 0 bis 23 vor. Der Maximalwert ist wiederum aus dem CrepidoAllietum doku-mentiert. Insgesamt sind die Flechten jene Artengruppe mit den geringsten mittleren Artendichten auf $4 \mathrm{~m}^{2}$ (Tab. 28). 


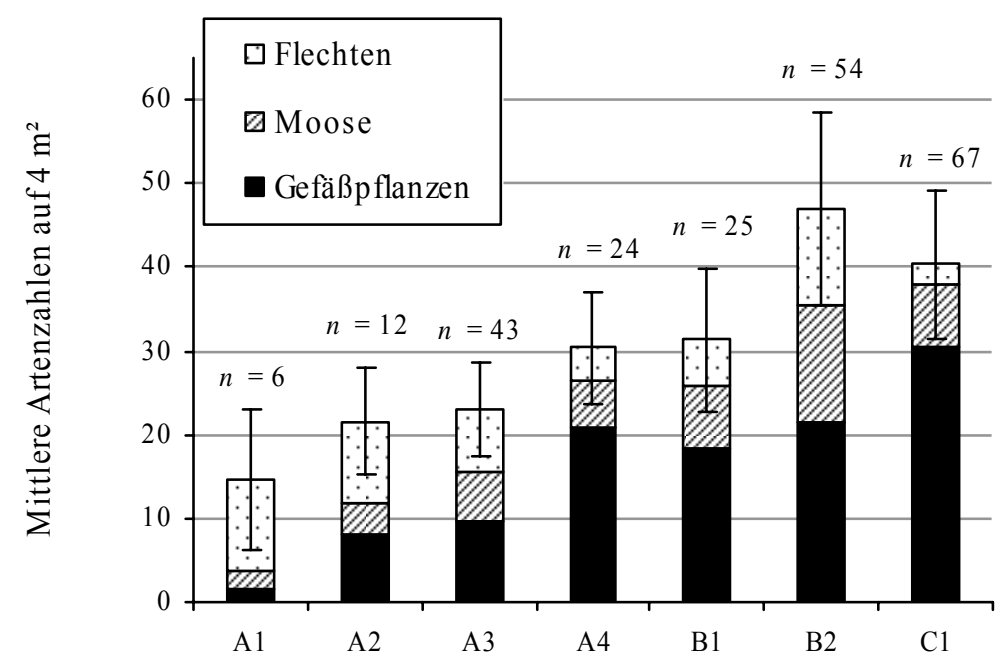

Abb. 1: Mittlere Gesamt- und Gruppenartenzahlen auf $4 \mathrm{~m}^{2}$ sowie deren Standardabweichung in den unterschiedlichen Trockenrasengesellschaften auf Saaremaa.

Tab. 28: Übersicht der Gesamt- und Gruppenartendichten auf $4 \mathrm{~m}^{2}$, bezogen auf sämtliche Aufnahmen ( $n=$ Anzahl der Aufnahmen, $\varnothing=$ arithmetisches Mittel, $\mathrm{s}=$ Standardabweichung).

\begin{tabular}{|l||c|c|c|c|}
\hline$n=231$ & $\begin{array}{c}\text { Gesamt- } \\
\text { artenzahl }\end{array}$ & $\begin{array}{c}\text { Artenzahl } \\
\text { Gefäß- } \\
\text { pflanzen }\end{array}$ & $\begin{array}{c}\text { Artenzahl } \\
\text { Moose }\end{array}$ & $\begin{array}{c}\text { Artenzahl } \\
\text { Flechten }\end{array}$ \\
\hline \hline$\varnothing$ & 35,3 & 20,3 & 8,1 & 6,6 \\
\hline $\mathrm{s}$ & 13,1 & 10,4 & 4,9 & 5,6 \\
\hline Minimum & 1,0 & 1,0 & 0,0 & 0,0 \\
\hline Maximum & 71,0 & 47,0 & 27,0 & 23,0 \\
\hline
\end{tabular}

Beim Vergleich der auf Basis der Einzelaufnahmen errechneten mittleren Artendichte auf $4 \mathrm{~m}^{2}$ ergaben sich drei homogene Gruppen: sie ist in den Sedo-Scleranthenea am höchsten, gefolgt von den Festuco-Brometea und am niedrigsten in den Koelerio-Corynephorenea (Tab. 29).

Insgesamt ist das Crepido-Allietum die artenreichste, das Caricetum arenariae die artenärmste Assoziation. In der Unterklasse Koelerio-Corynephorenea weisen die Bestände des SilenoFestucetum die höchste mittlere Artendichte auf $4 \mathrm{~m}^{2}$ auf. Das Helichryso-Jasionetum und das Festucetum polesicae liegen bezüglich der mittleren Artendichten nah beieinander. Das Cladonio-Sedetum ist die artenärmste Trockenrasenassoziation der Unterklasse SedoScleranthenea (Tab. 30).

Eine eindeutige Trennung in homogene Gruppen lässt sich im Hinblick auf die Gefäßpflanzenartendichten auf $4 \mathrm{~m}^{2}$ sowohl auf Hauptgruppen-, als auch auf Assoziationsebene feststellen. Die höchsten Werte erreichen dabei auf beiden Ebenen die Halbtrockenrasen (Tab. 29, Tab. 30). Die Unterklasse Koelerio-Corynephorenea ist deutlich am unteren Ende der Skala angesiedelt, während in den Sedo-Scleranthenea mittlere Werte erreicht werden (Tab. 29). Die mit Abstand niedrigsten Werte kommen im Caricetum arenariae vor. Das Helichryso-Jasionetum und Festucetum polesicae stehen sich auch bezüglich der mittleren Gefäßpflanzenartendichten sehr nahe und bilden eine Gruppe, während sich das Sileno-Festucetum auf einer Ebene mit den beiden Felsgrusgesellschaften einreiht (Tab. 30). 
Auf Hauptguppenebene lassen sich bezüglich der Moosartendichten auf $4 \mathrm{~m}^{2}$ drei Gruppen unterscheiden. Die Bestände der Unterklasse Koelerio-Corynephorenea weisen die niedrigsten und jene der Sedo-Scleranthenea die höchsten Werte auf (Tab. 29). Auf Assoziationsebene liegt das Crepido-Allietum deutlich vor den restlichen Trockenrasengesellschaften. Im unteren Bereich sind das Caricetum arenariae und das Helichryso-Jasionetum in einer Gruppe vereint. Im Cladonio-Sedetum kommen im Durchschnitt ebenso viele Moosarten auf $4 \mathrm{~m}^{2}$ vor wie in der Helictotrichon-Gesellschaft.

In den Sedo-Scleranthenea kommen ebenfalls die höchsten Flechtenartendichten vor. Die mittleren Moosartenzahlen auf $4 \mathrm{~m}^{2}$ liegen jedoch in den Koelerio-Corynephorenea nur geringfügig unter jenen der Unterklasse Sedo-Scleranthenea (Tab. 29). Auf Assoziationsebene bilden das Helichryso-Jasionetum, das Caricetum arenariae sowie das Crepido-Allietum die flechtenartenreichste Gruppe. Die Werte in den Halbtrockenrasen liegen deutlich unter denen der übrigen Trockenrasengesellschaften (Tab. 30).

Tab. 29: Ergebnisse der Post-hoc-Mehrfachvergleiche (Tukey-HSD) für die Gesamt- und Gruppenartendichten auf $4 \mathrm{~m}^{2}$ in den unterschiedlichen Hauptgruppen der Trockenrasen auf Saaremaa. Übereinstimmende Buchstaben kennzeichnen jeweils homogene Gruppen von Hauptgruppen bezüglich der Artendichte auf Basis eines $95 \%$-Konfidenzintervalles $(n=$ Anzahl).

\begin{tabular}{|l|l||c|c|c|c|c|c|c|c|}
\hline & $n$ & $\begin{array}{c}\text { Mittlere } \\
\text { Gesamt- } \\
\text { artenzahl }\end{array}$ & $\begin{array}{c}\text { Homogene } \\
\text { Gruppen } \\
\text { Gesamt- } \\
\text { artenzahl }\end{array}$ & $\begin{array}{c}\text { Mittlere } \\
\text { Artenzahl } \\
\text { Gefäß- } \\
\text { pflanzen }\end{array}$ & $\begin{array}{c}\text { Homogene } \\
\text { Gruppen } \\
\text { Gefäß- } \\
\text { pflanzen- } \\
\text { artenzahl }\end{array}$ & $\begin{array}{c}\text { Mittlere } \\
\text { Artenzahl } \\
\text { Moose }\end{array}$ & $\begin{array}{c}\text { Homogene } \\
\text { Gruppen } \\
\text { Moos- } \\
\text { artenzahl }\end{array}$ & $\begin{array}{c}\text { Mittlere } \\
\text { Artenzahl } \\
\text { Flechten }\end{array}$ & $\begin{array}{c}\text { Homogene } \\
\text { Gruppen } \\
\text { Flechten- } \\
\text { artenzahl }\end{array}$ \\
\hline \hline Koelerio-Corynephorenea & 85 & 24,3 & $\mathrm{a}$ & 12,1 & $\mathrm{a}$ & 5,1 & $\mathrm{a}$ & 7,2 & $\mathrm{~b}$ \\
\hline Sedo-Scleranthenea & 79 & 42,6 & $\mathrm{~b}$ & 20,5 & $\mathrm{~b}$ & 12,0 & $\mathrm{c}$ & 9,5 & $\mathrm{c}$ \\
\hline Festuco-Brometea & 67 & 40,5 & $\mathrm{~b}$ & 30,5 & $\mathrm{c}$ & 7,5 & $\mathrm{~b}$ & 2,4 & $\mathrm{a}$ \\
\hline
\end{tabular}

Tab. 30: Ergebnisse der Post-hoc-Mehrfachvergleiche (Tukey-HSD) für die Gesamt- und Gruppenartendichten auf $4 \mathrm{~m}^{2}$ in den unterschiedlichen Trockenrasentypen auf Saaremaa. Übereinstimmende Buchstaben kennzeichnen jeweils homogene Gruppen von Gesellschaften bezüglich der Artendichte auf Basis eines 95 \%Konfidenzintervalles $(n=$ Anzahl).

\begin{tabular}{|c|c|c|c|c|c|c|c|c|c|}
\hline & $n$ & $\begin{array}{l}\text { Mittlere } \\
\text { Gesamt- } \\
\text { artenzahl }\end{array}$ & $\begin{array}{c}\text { Homogene } \\
\text { Gruppen } \\
\text { Gesamt- } \\
\text { artenzahl }\end{array}$ & $\begin{array}{c}\text { Mittlere } \\
\text { Artenzahl } \\
\text { Gefäß- } \\
\text { pflanzen }\end{array}$ & $\begin{array}{c}\text { Homogene } \\
\text { Gruppen } \\
\text { Gefäß- } \\
\text { pflanzen- } \\
\text { artenzahl } \\
\end{array}$ & $\begin{array}{l}\text { Mittlere } \\
\text { Artenzahl } \\
\text { Moose }\end{array}$ & $\begin{array}{c}\text { Homogene } \\
\text { Gruppen } \\
\text { Moos- } \\
\text { artenzahl }\end{array}$ & $\begin{array}{c}\text { Mittlere } \\
\text { Artenzahl } \\
\text { Flechten }\end{array}$ & $\begin{array}{c}\text { Homogene } \\
\text { Gruppen } \\
\text { Flechten- } \\
\text { artenzahl }\end{array}$ \\
\hline Caricetum arenariae & 6 & 14,7 & $\mathrm{a}$ & 1,5 & $\mathrm{a}$ & 2,3 & $\mathrm{a}$ & 10,8 & $\mathrm{c}$ \\
\hline Helichryso-Jasionetum & 12 & 21,6 & $\mathrm{a}$ & 8,1 & $\mathrm{~b}$ & 3,6 & $\mathrm{a}$ & 9,9 & $\mathrm{c}$ \\
\hline Festucetum polesicae & 43 & 23,1 & $a, b$ & 9,7 & $\mathrm{~b}$ & 5,8 & $a, b$ & 7,7 & $\mathrm{~b}, \mathrm{c}$ \\
\hline Sileno-Festucetum & 24 & 30,4 & $\mathrm{~b}$ & 21,0 & $\mathrm{c}$ & 5,3 & $a, b$ & 4,1 & $a, b$ \\
\hline Cladonio-Sedetum & 25 & 31,5 & $\mathrm{~b}$ & 18,5 & $\mathrm{c}$ & 7,5 & $\mathrm{~b}$ & 5,3 & $\mathrm{a}, \mathrm{b}$ \\
\hline Crepido-Allietum & 54 & 47,7 & $\mathrm{c}$ & 21,4 & $\mathrm{c}$ & 14,1 & $\mathrm{c}$ & 11,4 & $\mathrm{c}$ \\
\hline Helictotrichon- Ges. & 67 & 40,5 & $\mathrm{c}$ & 30,5 & $\mathrm{~d}$ & 7,5 & $\mathrm{~b}$ & 2,4 & $\mathrm{a}$ \\
\hline
\end{tabular}

Ferner sind Unterschiede bei den Anteilen der Artengruppen an der Gesamtartenzahl in den verschiedenen Trockenrasentypen zu erkennen (Abb. 2). 


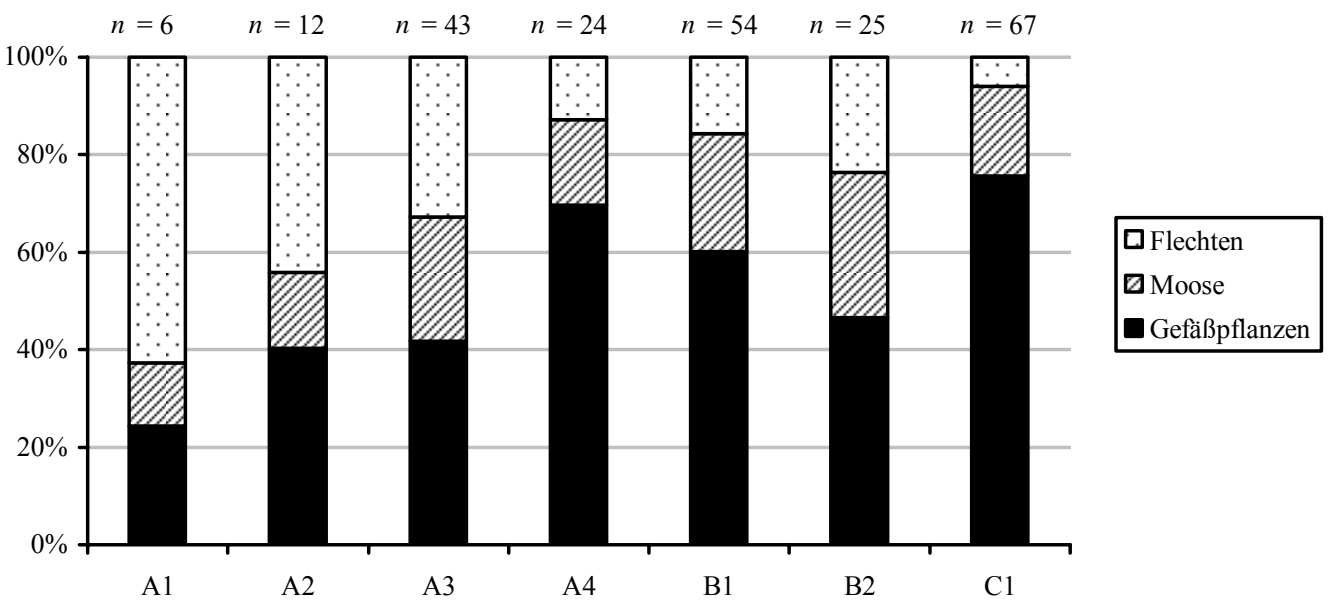

Abb. 2: Durchschnittliche Anteile der Artengruppen am Gesamtartenreichtum auf $4 \mathrm{~m}^{2}$ in den unterschiedlichen Trockenrasengesellschaften auf Saaremaa.

Die Spanne des prozentualen Anteils der Gefäßpflanzen am Gesamtartenreichtum auf $4 \mathrm{~m}^{2}$ beträgt 6-100\%, jene der Moose 0-48 \% und die der Flechten 0-78 \%. Im Durchschnitt haben die Gefäßpflanzen mit etwa zwei Dritteln den höchsten prozentualen Anteil am Gesamtartenreichtum. Die Moose und Flechten liegen etwa gleichauf mit ungefähr jeweils einem Fünftel am Gesamtartenreichtum (Tab. 31).

Tab. 31: Übersicht der Gesamt- und Gruppenartendichten auf $4 \mathrm{~m}^{2}$ bezogen auf sämtliche Aufnahmen ( $n=$ Anzahl der Aufnahmen, $\varnothing=$ arithmetisches Mittel, $\mathrm{s}=$ Standardabweichung).

\begin{tabular}{|l||c|c|c|}
\hline$n=231$ & $\begin{array}{c}\text { Anteil } \\
\text { Gefäßpflanzen }\end{array}$ & Anteil Moose & Anteil Flechten \\
\hline \hline$\varnothing$ & 57 & 22 & 20 \\
\hline $\mathrm{s}$ & 21 & 9 & 18 \\
\hline Minimum & 6 & 0 & 0 \\
\hline Maximum & 100 & 48 & 78 \\
\hline
\end{tabular}

Betrachtet man auf Hauptgruppenebene den mittleren Anteil der Gefäßpflanzen am Gesamtartenreichtum auf $4 \mathrm{~m}^{2}$, bilden die Bestände Halbtrockenrasen mit höheren Werten als die beiden anderen Hauptgruppen eine deutlich isolierte Gruppe. Bezogen auf den Moosanteil liegen die Bestände der Unterklasse Sedo-Scleranthenea über jenen der KoelerioCorynephorenea sowie der Klasse Festuco-Brometea, wobei die beiden Letztgenannten in einer Gruppe vereint sind. Hinsichtlich des Anteils der Flechten am Gesamtartenreichtum grenzen sich die drei Hauptgruppen deutlich voneinander ab. Der höchste Wert ist mit etwa einem Drittel in den Koelerio-Corynephorenea festzustellen, in den Sedo-Scleranthenea beträgt er ein Fünftel und die Helictotrichon-Gesellschaft ist am unteren Ende der Skala angesiedelt (Tab. 32).

Auf Assoziationsebene weist die Helictotrichon-Gesellschaft den höchsten mittleren Anteil an Gefäßpflanzen- und den niedrigsten an Flechtenarten auf. Im im Caricetum arenariae hingegen ist eine umgekehrte Verteilung der Anteile dieser Artengruppen am Gesamtartenreichtum auf $4 \mathrm{~m}^{2} \mathrm{zu}$ erkennen. Das Helichryso-Jasionetum und das Festucetum polesicae bilden bezogen auf den Anteil der Gefäßpflanzen isolierte Gruppe und liegen über dem Caricetum arenariae. Der höchste Moosartenanteil am Gesamtartenreichtum ist im Crepido-Allietum zu finden. Das Caricetum arenariae und das Helichryso-Jasionetum liegen 
hingegen in einer Gruppe vereint am unteren Ende der Skala. Den zweithöchsten Anteil am Gesamtartenreichtum haben die Flechten im Helichryso-Jasionetum, dessen Bestände eine homogene Gruppe bilden und somit zwischen jenen des Caricetum arenariae und des Festucetum polesicae angesiedelt sind (Tab. 33).

Tab. 32: Arithmetische Mittel (Ø), Standardabweichungen (s) und Ergebnisse der Post-hoc-Mehrfachvergleiche (Tukey-HSD) für die prozentualen Anteile der Artengruppen am Gesamtartenreichtum auf $4 \mathrm{~m}^{2}$ in den unterschiedlichen Hauptgruppen der Trockenrasen auf Saaremaa. Übereinstimmende Buchstaben kennzeichnen jeweils homogene Gruppen bezüglich der Anteile der Artengruppen am Gesamtartenreichtum auf Basis eines $95 \%$-Konfidenzintervalles ( $n=$ Anzahl der Aufnahmen).

\begin{tabular}{|c|c|c|c|c|c|c|c|c|c|c|}
\hline & \multirow[t]{2}{*}{$n$} & \multicolumn{2}{|c|}{$\begin{array}{l}\text { Anteil Gefäß- } \\
\text { pflanzen [\%] }\end{array}$} & \multirow{2}{*}{$\begin{array}{c}\text { Homogene } \\
\text { Gruppen } \\
\text { Anteil Gefäß- } \\
\text { pflanzen }\end{array}$} & \multicolumn{2}{|c|}{$\begin{array}{c}\text { Anteil Moose } \\
{[\%]}\end{array}$} & \multirow[t]{2}{*}{$\begin{array}{c}\text { Homogene } \\
\text { Gruppen } \\
\text { Anteil Moose }\end{array}$} & \multicolumn{2}{|c|}{$\begin{array}{c}\text { Anteil } \\
\text { Flechten [\%] }\end{array}$} & \multirow{2}{*}{$\begin{array}{c}\begin{array}{c}\text { Homogene } \\
\text { Gruppen } \\
\text { Anteil Flechten }\end{array} \\
\end{array}$} \\
\hline & & $\varnothing$ & $\mathrm{s}$ & & $\varnothing$ & $\mathrm{s}$ & & $\varnothing$ & $\mathrm{s}$ & \\
\hline Koelerio-Corynephorenea & 85 & 48 & 22 & $\mathrm{a}$ & 21 & 9 & $\mathrm{a}$ & 31 & 20 & $\mathrm{c}$ \\
\hline Sedo-Scleranthenea & 79 & 50 & 13 & $\mathrm{a}$ & 28 & 8 & $\mathrm{~b}$ & 21 & 11 & $\mathrm{~b}$ \\
\hline Festuco-Bometea & 67 & 75 & 12 & $\mathrm{~b}$ & 18 & 6 & $\mathrm{a}$ & 6 & 7 & $\mathrm{a}$ \\
\hline
\end{tabular}

Tab. 33: Arithmetische Mittel (Ø), Standardabweichungen (s) und Ergebnisse der Post-hoc-Mehrfachvergleiche (Tukey-HSD) für die prozentualen Anteile der Artengruppen am Gesamtartenreichtum auf $4 \mathrm{~m}^{2}$ in den unterschiedlichen Trockenrasentypen sowie den Hauptgruppen auf Saaremaa. Übereinstimmende Buchstaben kennzeichnen jeweils homogene Gruppen bezüglich der Anteile der Artengruppen am Gesamtartenreichtum auf Basis eines $95 \%$-Konfidenzintervalles ( $n=$ Anzahl der Aufnahmen).

\begin{tabular}{|c|c|c|c|c|c|c|c|c|c|c|}
\hline & \multirow[t]{2}{*}{$n$} & \multicolumn{2}{|c|}{$\begin{array}{l}\text { Anteil Gefäß- } \\
\text { pflanzen [\%] }\end{array}$} & \multirow{2}{*}{$\begin{array}{c}\text { Homogene } \\
\text { Gruppen } \\
\text { Anteil Gefäß- } \\
\text { pflanzen }\end{array}$} & \multicolumn{2}{|c|}{$\begin{array}{c}\text { Anteil Moose } \\
{[\%]}\end{array}$} & \multirow{2}{*}{$\begin{array}{c}\text { Homogene } \\
\text { Gruppen } \\
\text { Anteil } \\
\text { Moose } \\
\end{array}$} & \multicolumn{2}{|c|}{$\begin{array}{c}\text { Anteil } \\
\text { Flechten }[\%]\end{array}$} & \multirow{2}{*}{$\begin{array}{c}\text { Homogene } \\
\text { Gruppen } \\
\text { Anteil } \\
\text { Flechten }\end{array}$} \\
\hline & & $\varnothing$ & $\mathrm{S}$ & & $\varnothing$ & $\mathrm{s}$ & & $\varnothing$ & $\mathrm{s}$ & \\
\hline Caricetum arenariae & 6 & 24 & 37 & $\mathrm{a}$ & 13 & 8 & $\mathrm{a}$ & 63 & 31 & $\mathrm{e}$ \\
\hline Helichryso-Jasionetum & 12 & 40 & 20 & $\mathrm{~b}$ & 16 & 7 & $\mathrm{a}$ & 44 & 18 & $\mathrm{~d}$ \\
\hline Festucetum polesicae & 43 & 42 & 15 & $\mathrm{~b}$ & 25 & 8 & $\mathrm{c}, \mathrm{d}$ & 33 & 12 & $\mathrm{c}, \mathrm{d}$ \\
\hline Sileno-Festucetum & 24 & 70 & 12 & $\mathrm{~d}, \mathrm{e}$ & 18 & 6 & $a, b$ & 13 & 11 & $\mathrm{a}, \mathrm{b}$ \\
\hline Cladonio-Sedetum & 25 & 60 & 12 & $\mathrm{c}, \mathrm{d}$ & 24 & 6 & $\mathrm{~b}, \mathrm{c}, \mathrm{d}$ & 16 & 12 & $\mathrm{a}, \mathrm{b}$ \\
\hline Crepido-Allietum & 54 & 46 & 10 & $\mathrm{~b}, \mathrm{c}$ & 29 & 9 & $\mathrm{~d}$ & 23 & 10 & $\mathrm{~b}, \mathrm{c}$ \\
\hline Helictotrichon-Ges. & 67 & 75 & 12 & $\mathrm{e}$ & 18 & 6 & $\mathrm{a}, \mathrm{b}, \mathrm{c}$ & 6 & 7 & $\mathrm{a}$ \\
\hline
\end{tabular}

\subsection{Muster der Phythodiversität}

\subsubsection{Beziehungen zwischen Artenreichtum und abiotischen Umweltparametern}

Zur Klärung der Frage, welchen Einfluss die abiotischen Parameter auf den Gesamtartenreichtum auf $4 \mathrm{~m}^{2}$ haben, und ob der Artenreichtum der Artengruppen in unterschiedlicher Weise beeinflusst wird, habe ich zum einen schrittweise multiple lineare Regressionsanalysen und zum anderen einfache Regressionsstatistiken zur Kurvenanpassung durchgeführt. Letztere Methode dient dazu, Parameter, welche nicht in die multiplen Modelle eingeschlossen wurden, aber dennoch signifikante Zusammenhänge zum Artenreichtum aufweisen, verdeutlichen zu können. 
Die folgenden Parameter sowie deren quadratische Terme wurden als unabhängige Variablen in die schrittweise multiple lineare Regressionsanalysen aufgenommen:

- $\quad$ Überstauung

- Bodentiefgründigkeit

- Mikrorelief

- Skelett

- Deckung Gestein

- Humusgehalt
- $\mathrm{pH}-$ Wert

- Basensättigung

- Carbonatgehalt

- Höhe ü. NN

- Größe der Trockenrasengebiete

Den S-Wert und die Kationenaustauschkapazität sowie den H-Wert habe ich nicht in die Regressionsanalysen aufgenommen, da die ersten beiden Variablen stark mit dem Carbonatgehalt, die letztgenannte mit der Basensättigung co-korreliert sind $(r \geq 0,8)$ (Tab. 19).

Die Überstauung stellt in den erhaltenen Modellen für die Gesamt-, Moos- und Flechtenartendichte die einflussreichste Variable dar (Tab. 34, Tab. 36, Tab. 37). Zusätzlich zu den in das multiple Regressionsmodell für die Gesamtartendichte aufgenommenen Variablen ist ein signifikanter Zusammenhang mit unimodalem Kurvenverlauf (Optimumkurve) zu dem Gesteinsanteil, dem Humusgehalt sowie dem $\mathrm{pH}-$ Wert des Oberbodens festzustellen (Tab. 38). Bezüglich des erhaltenen Modells für die Gefäßpflanzenartendichte (Tab. 35) stellt der Gesteinsanteil die einflussreichste Variable dar. Nicht aufgenommen, jedoch signifikant korreliert sind die Parameter Humusgehalt, $\mathrm{pH}-$ Wert und Höhe ü. NN. Hinsichtlich dieser Parameter liegt eine Optimumkurve vor (Tab. 38). Die Parameter Gesteinsanteil, Humusgehalt, $\mathrm{pH}-$ Wert und Carbonatgehalt sind signifikant mit den Moosartendichten korreliert (Tab. 38), wurden jedoch nicht in das multiple Modell eingeschlossen (Tab. 36). In das erhaltene Modell für die Flechtenartendichte (Tab. 37) nicht aufgenommen und dennoch signifikant korreliert ist die Gesteinsdeckung sowie die Basensättigung (Tab. 38).

Deutliche Unterschiede zwischen den einzelnen Teilmodellen bezüglich der Werteverteilung von Parametern in Beziehung $\mathrm{zu}$ den Artendichten lassen sich beispielsweise bei der Bodentiefgründigkeit erkennen. Bezogen auf die Gesamt- und die Gefäßpflanzenartendichte liegt eine Optimumkurve, bezüglich der Flechtenartendichte eine Minimumkuve vor. Ebenso ist ein gegenläufiges Ergebnis bezüglich des Gesteinsanteils des Bodens festzustellen. Bezogen auf die Gesamtartendichte besteht ein linear negativer, hinsichtlich der Flechtenartendichte ein positiver Zusammenhang (Tab. 35, Tab. 37). Eine Optimumkurve zeichnet sich in der Werteverteilung zwischen der Tiefgründigkeit und der Gesamt- sowie Gefäßpflanzenartendichte ab (Tab. 34, Tab. 35), während bezüglich der Moosartendichte eine linear negative Beziehung zu erkennen ist (Tab. 36).

Tab. 34: Ergebnisse der schrittweisen multiplen linearen Regressionsanalyse mit der Gesamtartendichte als abhängige Variable und den erfassten abiotischen Parametern als unabhängige Variablen $(n=167)$. Die Variablen wurden in Abhängigkeit des Signifikanzniveaus ihres F-Wertes eingeschlossen $(p<0,05)$ oder entfernt $(p>0,1)$.

\begin{tabular}{|l||c|c|c|}
\hline Abhängige Variable: Gesamtartendichte auf $4 \mathrm{~m}^{2}$ & $r^{2}$ & $b$ & $p$ \\
\hline \hline Tiefgründigkeit $^{2}$ & & $-0,006$ & $<0,001$ \\
Mikrorelief & & 0,707 & $<0,001$ \\
Carbonatgehalt & 0,423 & $-0,237$ & 0,005 \\
Überstauung & & 5,624 & 0,006 \\
\hline
\end{tabular}


Tab. 35: Ergebnisse der schrittweisen multiplen linearen Regressionsanalyse mit der Gefäßpflanzenartendichte als abhängige Variable und den erfassten abiotischen Parametern als unabhängige Variablen $(n=167)$. Die Variablen wurden in Abhängigkeit des Signifikanzniveaus ihres F-Wertes eingeschlossen $(p<0,05)$ oder entfernt $(p>0,1)$.

\begin{tabular}{|l||c|c|c|}
\hline Abhängige Variable: Gefäßpflanzenartendichte auf $4 \mathrm{~m}^{2}$ & $r^{2}$ & $b$ & $p$ \\
\hline \hline Tiefgründigkeit $^{2}$ & & $-0,005$ & $<0,001$ \\
Größe der Trockenrasengebiete & & $<0,001$ & $<0,001$ \\
Gesteinsanteil & 0,482 & $-0,088$ & $<0,001$ \\
Größe der Trockenrasengebiete $^{2}$ & & $<0,001$ & 0,001 \\
\hline
\end{tabular}

Tab. 36: Ergebnisse der schrittweisen multiplen linearen Regressionsanalyse mit der Moosartendichte als abhängige Variable und den erfassten abiotischen Parametern als unabhängige Variablen $(n=167)$. Die Variablen wurden in Abhängigkeit des Signifikanzniveaus ihres F-Wertes eingeschlossen $(p<0,05)$ oder entfernt $(p>0,1)$.

\begin{tabular}{|l||c|c|c|}
\hline Abhängige Variable: Moosartendichte auf $4 \mathrm{~m}^{2}$ & $r^{2}$ & $b$ & $p$ \\
\hline \hline Überstauung & & 4,820 & $<0,001$ \\
Mikrorelief & 0,479 & 0,314 & $<0,001$ \\
Tiefgründigkeit & & $-0,049$ & $<0,001$ \\
\hline
\end{tabular}

Tab. 37: Ergebnisse der schrittweisen multiplen linearen Regressionsanalyse mit der Flechtenartendichte als abhängige Variable und den erfassten abiotischen Parametern als unabhängige Variablen $(n=167)$. Die Variablen wurden in Abhängigkeit des Signifikanzniveaus ihres F-Wertes eingeschlossen $(p<0,05)$ oder entfernt $(p>0,1)$.

\begin{tabular}{|c|c|c|c|}
\hline Abhängige Variable: Flechtenartendichte auf $4 \mathrm{~m}^{2}$ & $r^{2}$ & $b$ & $p$ \\
\hline Größe der Trockenrasengebiete & & $<0,001$ & $<0,001$ \\
\hline Gesteinsanteil & & 0,072 & $<0,001$ \\
\hline Carbonatgehalt & 0,378 & $-0,170$ & $<0,001$ \\
\hline Überstauung & & 2,870 & 0,001 \\
\hline Mikrorelief $^{2}$ & & 0,010 & 0,004 \\
\hline
\end{tabular}


Tab. 38: Ergebnisse der einfachen Regressionsstatistiken zur Kurvenanpassung mit der Gesamt-, Gefäßpflanzen, Moos- sowie Flechtenartendichte als abhängige Variable und den erfassten abiotischen Parametern als unabhängige Variablen $(n=167)$. Auf dem Niveau $p<0,05$ signifikante Zusammenhänge sind fett gesetzt (Berechnung der Werte: Bodentiefgründigkeit, Größe der Trockenrasengebiete, Gesteinsanteil vgl. Abschnitt 3.1.2; Carbonatgehalt vgl. Abschnitt 3.3.2; $p=$ Signifikanzniveau, Regressionskoeffizienten: $b_{0}=$ Konstante, $b_{1}=$ linearer Term, $b_{2}=$ quadratischer Term).

\begin{tabular}{|c|c|c|c|c|c|c|c|c|c|}
\hline \multirow[t]{2}{*}{ Abhängige Variable ... } & & \multicolumn{2}{|c|}{ Gesamtartendichte } & \multicolumn{2}{|c|}{$\begin{array}{l}\text { Gefäßpflanzen- } \\
\text { artendichte }\end{array}$} & \multicolumn{2}{|c|}{ Moosartendichte } & \multicolumn{2}{|c|}{ Flechtenartendichte } \\
\hline & & & $p$ & & $p$ & & $p$ & & $p$ \\
\hline Überstauung & $\begin{array}{l}b_{0} \\
b_{1} \\
b_{2}\end{array}$ & $\begin{array}{c}31,259 \\
11,647 \\
-\end{array}$ & $<0,001$ & $\begin{array}{c}19,874 \\
0,876 \\
-\end{array}$ & 0,649 & $\begin{array}{c}6,17 \\
6,298 \\
-\end{array}$ & $<0,001$ & $\begin{array}{c}5,104 \\
3,865 \\
-\end{array}$ & $<0,001$ \\
\hline Bodentiefgründigkeit & $\begin{array}{l}b_{0} \\
b_{1} \\
b_{2}\end{array}$ & $\begin{array}{c}36,598 \\
0,365 \\
-0,013 \\
\end{array}$ & $<0,001$ & $\begin{array}{c}\mathbf{1 7 , 2 1 1} \\
0,959 \\
-\mathbf{0 , 0 2 1} \\
\end{array}$ & $<0,001$ & $\begin{array}{c}9,442 \\
-0,092 \\
- \\
\end{array}$ & $<0,001$ & $\begin{array}{c}9,254 \\
-0,453 \\
0,008 \\
\end{array}$ & 0,001 \\
\hline Mikrorelief [cm] & $\begin{array}{l}b_{0} \\
b_{1} \\
b_{2}\end{array}$ & $\begin{array}{c}27,596 \\
1,509 \\
-0,026\end{array}$ & $<0,001$ & $\begin{array}{c}18,821 \\
0,267 \\
-\end{array}$ & 0,139 & $\begin{array}{c}4,550 \\
0,748 \\
-0,015\end{array}$ & $<0,001$ & $\begin{array}{c}4,766 \\
0,236 \\
- \\
\end{array}$ & 0,016 \\
\hline Gesteinsanteil & $\begin{array}{l}b_{0} \\
b_{1} \\
b_{2}\end{array}$ & $\begin{array}{c}30,931 \\
0,490 \\
-0,006 \\
\end{array}$ & $<0,001$ & $\begin{array}{c}20,160 \\
0,096 \\
-0,002 \\
\end{array}$ & 0,199 & $\begin{array}{c}6,324 \\
0,189 \\
-0,002 \\
\end{array}$ & $<0,001$ & $\begin{array}{r}4,361 \\
0,187 \\
-0,002 \\
\end{array}$ & $<0,001$ \\
\hline Deckung Gestein [\%] & $\begin{array}{l}b_{0} \\
b_{1} \\
b_{2}\end{array}$ & $\begin{array}{c}32,173 \\
0,341 \\
-0,004 \\
\end{array}$ & 0,054 & $\begin{array}{c}20,514 \\
-0,044 \\
- \\
\end{array}$ & 0,227 & $\begin{array}{c}6,733 \\
0,139 \\
-0,002 \\
\end{array}$ & 0,093 & $\begin{array}{c}4,815 \\
0,220 \\
-0,002 \\
\end{array}$ & $<0,001$ \\
\hline Humusgehalt [\%] & $\begin{array}{l}b_{0} \\
b_{1} \\
b_{2} \\
\end{array}$ & $\begin{array}{c}24,836 \\
1,750 \\
-0,031 \\
\end{array}$ & $<0,001$ & $\begin{array}{c}14,040 \\
1,203 \\
-0,021 \\
\end{array}$ & $<0,001$ & $\begin{array}{r}\mathbf{4 , 9 5 8} \\
\mathbf{0 , 4 8 3} \\
\mathbf{- 0 , 0 0 8} \\
\end{array}$ & $<0,001$ & $\begin{array}{c}5,801 \\
0,023 \\
-0,001 \\
\end{array}$ & 0,944 \\
\hline pH-Wert & $\begin{array}{l}b_{0} \\
b_{1} \\
b_{2} \\
\end{array}$ & $\begin{array}{c}-124,871 \\
45,338 \\
-3,207 \\
\end{array}$ & 0,022 & $\begin{array}{c}-131,853 \\
45,729 \\
-3,383 \\
\end{array}$ & $\mathbf{0 , 0 1 2}$ & $\begin{array}{c}-28,523 \\
9,486 \\
-0,619 \\
\end{array}$ & 0,016 & $\begin{array}{c}36,183 \\
-9,993 \\
0,793 \\
\end{array}$ & 0,214 \\
\hline Basensättigung [\%] & $\begin{array}{l}b_{0} \\
b_{1} \\
b_{2}\end{array}$ & $\begin{array}{c}43,950 \\
-0,537 \\
0,004\end{array}$ & 0,682 & $\begin{array}{c}-56,426 \\
1,926 \\
-0,012\end{array}$ & 0,687 & $\begin{array}{c}-4,328 \\
0,118 \\
-\end{array}$ & 0,207 & $\begin{array}{c}-17,711 \\
0,238 \\
-\end{array}$ & $\mathbf{0 , 0 3 9}$ \\
\hline Carbonatgehalt & $\begin{array}{l}b_{0} \\
b_{1} \\
b_{2} \\
\end{array}$ & $\begin{array}{c}32,305 \\
0,135 \\
- \\
\end{array}$ & 0,169 & $\begin{array}{c}19,298 \\
0,079 \\
- \\
\end{array}$ & 0,304 & $\begin{array}{c}6,351 \\
0,219 \\
-0,005 \\
\end{array}$ & 0,028 & $\begin{array}{r}6,135 \\
-0,013 \\
-0,001 \\
\end{array}$ & 0,676 \\
\hline Höhe ü. NN [m] & $\begin{array}{l}b_{0} \\
b_{1} \\
b_{2} \\
\end{array}$ & $\begin{array}{c}30,010 \\
0,886 \\
-\mathbf{0 , 0 2 3} \\
\end{array}$ & $\mathbf{0 , 0 0 7}$ & $\begin{array}{c}\mathbf{1 7 , 0 1 8} \\
0,690 \\
-\mathbf{0 , 0 1 6} \\
\end{array}$ & 0,013 & $\begin{array}{c}6,599 \\
0,206 \\
-0,006 \\
\end{array}$ & 0,092 & $\begin{array}{c}6,376 \\
-0,064 \\
- \\
\end{array}$ & 0,118 \\
\hline $\begin{array}{l}\text { Größe der } \\
\text { Trockenrasengebiete }\end{array}$ & $\begin{array}{l}b_{0} \\
b_{1} \\
b_{2}\end{array}$ & $\begin{array}{l}36,392 \\
-0,001 \\
<0,001\end{array}$ & 0,065 & $\begin{array}{c}26,116 \\
-0,001 \\
<0,001\end{array}$ & $<0,001$ & $\begin{array}{c}6,957 \\
<0,001 \\
<0,001\end{array}$ & 0,614 & $\begin{array}{c}\mathbf{3 , 1 7 3} \\
<\mathbf{0 , 0 0 1} \\
<\mathbf{0 , 0 0 1}\end{array}$ & $<0,001$ \\
\hline
\end{tabular}




\subsubsection{Beziehungen zwischen Artenreichtum und biotischen Umweltparametern}

Um die Frage zu klären, welchen Einfluss die untersuchten biotischen Parameter auf den Gesamtartenreichtum haben, und ob sich daraus Indizien für eine interspezifische Konkurrenz ableiten lassen, habe ich die Deckungsgrade der Artengruppen mit der Restvarianz - den Residuen - der schrittweisen multiplen linearen Regressionsanalysen korreliert. Dabei sind folgende Zusammenhänge zu erkennen (Tab. 39):

Die Residuen der Regressionsanalyse bezogen auf die Gefäßpflanzenartenzahlen sind hochsignifikant positiv mit den Deckungsgraden der Gefäßpflanzen korreliert. Außerdem ist ein hochsignifikant negativer Zusammenhang zwischen diesen Residuen und den Deckungsgraden der Flechten festzustellen.

Zwischen den Residuen der Regressionsanalyse bezogen auf die Moosartenzahlen und den Deckungsgraden der unterschiedlichen Artengruppen besteht kein signifikanter Zusammenhang.

Die Residuen der Regressionsanalyse bezogen auf die Flechtenartenzahlen sind signifikant negativ mit den Deckungsgraden der Gefäßpflanzen korreliert. Hochsignifikant positiv hängen diese Residuen und die Deckungsgrade der Flechten zusammen.

Tab. 39: Bivariate Korrelationsanalysen (Pearson) zwischen den Residuen der schrittweisen multiplen linearen Regressionsanalysen und den erfassten Strukturparametern $(n=167)$. Hochsignifikante Zusammenhänge $(p<0,01)$ sind fett gedruckt und dunkelgrau, auf dem Niveau $p<0,05$ signifikante Zusammenhänge hellgrau unterlegt.

\begin{tabular}{|l|c|c|c|c|}
\hline Residuen der ... & & $\begin{array}{c}\text { Gefäßpflanzen- } \\
\text { artenzahlen }\end{array}$ & Moosartenzahlen & $\begin{array}{c}\text { Flechten- } \\
\text { artenzahlen }\end{array}$ \\
\hline Deckung Gefäßpflanzen & $r$ & $\mathbf{0 , 2 8 6}$ & 0,084 & $\mathbf{- 0 , 2 4 1}$ \\
& $p$ & $<0,001$ & 0,279 & 0,002 \\
\hline \multirow{2}{*}{ Deckung Moose } & $r$ & 0,083 & 0,109 & 0,046 \\
\hline \multirow{2}{*}{ Deckung Flechten } & $p$ & 0,284 & 0,162 & 0,558 \\
\hline & $r$ & $-0,196$ & 0,102 & $\mathbf{0 , 5 2 0}$ \\
\hline
\end{tabular}




\section{Diskussion}

Im Folgenden wird die Stellung der estnischen Trockenrasen im überregionalen Vergleich erörtert, die Übereinstimmung der Trockenrasenbestände mit den in Anhang I der FFHRichtlinie aufgeführten Lebensraumtypen besprochen, neue und interessante Pflanzenfunde präsentiert und die Muster der Phytodiversität hinsichtlich der Auswirkungen von abiotischen, biotischen und historischen Parametern diskutiert.

\subsection{Klassifikation und Charakterisierung der estnischen Trockenrasen im über- regionalen Vergleich}

Die syntaxonomische Einteilung der estnischen Trockenrasen auf höherer Ebene richtet sich aus pragmatischen Gründen überwiegend nach DENGLER et al. (2003), DENGLER (2004a, 2004b) sowie DENGLER \& LÖBEL (2006), da diese umfangreiche Datensätze ausgewertet und in einen überregionalen Kontext gestellt haben.

Es steht die Frage im Vordergrund, wie sich die Bestände der estnischen Trockenrasen in dieses bestehende und überregional gültige System einordnen lassen.

Daher wird im Folgenden zunächst die Einordnung der jeweiligen Assoziation nach DENGLER et al. (2003), Dengler (2004a, 2004b) bzw. DenGLER \& LÖBEL (2006) dargestellt. Zusätzlich gebe ich davon abweichende oder übereinstimmende Einordnungsvorschläge weiterer Autoren an, deren System ebenfalls überregional gültig ist. Daran anschließend begründe ich die in dieser Arbeit vorgenommene Einordnung in oben genanntes System. Zudem wird erörtert, ob den Assoziationen entsprechende Einheiten bereits in der verfügbaren Literatur über die estnische Vegetation erwähnt wurden und inwieweit diese den hier aufgeführten Assoziationen entsprechen. Des Weiteren werden die floristischen und ökologischen Unterschiede bzw. Gemeinsamkeiten der Bestände des Untersuchungsgebietes zu jenen anderer Regionen herausgearbeitet.

In Tab. 40 sind die sieben unterschiedenen Trockenrasengesellschaften, ihre gültigen Namen sowie die übergeordneten Syntaxa aufgelistet. Weitere im Text erwähnte Syntaxa sind bei der ersten Nennung mit dem entsprechenden Autorenzitat versehen. Auf eine Nennung von Synonymen verzichte ich. Sie können Dengler (2004a, 2004b) bzw. Dengler \& LöBel (2006) entnommen werden.

\subsubsection{Höhere Syntaxa}

Nach Dengler et al. (2003) und Dengler (2004a, 2004b) werden die beiden Klassen Koelerio-Corynephoretea s. 1. und Festuco-Brometea unterschieden und erstgenannte zusätzlich in zwei Unterklassen getrennt. Diese drei Hauptgruppen sind auf Saaremaa eindeutig durch eigene Charakter- und Differenzialarten gekennzeichnet. Ferner sind die beiden Unterklassen durch eine Reihe von gemeinsamen Arten verbunden und von der Klasse Festuco-Brometea getrennt (Tab. A).

Ähnlich den Trockenrasen auf Öland (vgl. DeNGLER \& LöBEL 2006) zeigen Arten wie Festuca ovina und Thymus serpyllum, die in Mitteleuropa auf die Klasse KoelerioCorynephoretea beschränkt sind (z. B. DENGLER 2001b, DenGLer 2004a), bzw. zweitere auf die Unterklasse Koelerio-Corynephorenea (KORNECK 1975), auf Saaremaa eine weite Amplitude und kommen ebenso häufig in den Festuco-Brometea vor. Das Gleiche gilt für die andernorts typische Festuco-Brometea-Art Thuidium abietinum (DENGLER 2004b) (Tab. A). 


\subsubsection{Koelerio-Corynephoretea}

DENGLER et al. (2003) und DENGLER (2004a) nehmen eine Zweiteilung in die Unterklassen Koelerio-Corynephorenea (Sandtrockenrasen) und Sedo-Scleranthenea (Felsgrusfluren) vor, da einerseits in beiden Unterklassen gemeinsame Arten vorkommen, die sie gegenüber anderen Xerothermgesellschaften differenzieren, andererseits jedoch beide Gesellschaftsgruppen durch eigene Charakterarten gut voneinander abgegrenzt sind. Verschiedene Autoren (z. B. Krausch 1962, Ротt 1995, Schubert et al. 2001) spalten die Klasse KoelerioCorynephoretea s. 1. hingegen in drei, DIERßEN (1996) in zwei Ordnungen auf. Die Einteilung von letztgenanntem Autor, welcher sämtliche Sandtrockenrasen in der ersten und die Felsgrusfluren in der zweiten Ordnung zusammenfasst, kommt somit dem System von DENGLER et al. (2003) bzw. DENGLER (2004a) bereits sehr nahe.

Erstgenannter Vorschlag ließ sich am eigenen Aufnahmematerial anhand zahlreicher Charakter- und Differenzialarten der beiden Unterklassen (Tab. A) nachvollziehen und wurde als Gliederungskonzept verwendet.

Die Klasse Koelerio-Corynephoretea umfasst in dieser Arbeit somit die KoelerioCorynephorenea (Sandtrockenrasen) und die Sedo-Scleranthenea (Felsgrusfluren).

\subsubsection{Koelerio-Corynephorenea}

DENGLER et al. (2003) teilen die Unterklasse Koelerio-Corynephorenea in sechs Ordnungen. Andere Autoren (z. B. PotT 1995, Schubert et al. 2001) fassen sie in zwei Ordnungen zusammen, während sich DIERßEN (1996) auf eine Ordnung beschränkt, in welche er sämtliche Sandtrockenrasen einordnet.

Von erstgenannter Einteilung habe ich im Untersuchungsgebiet die drei Ordnungen Corynephoretalia canescentis (A.a), Sedo acris-Festucetalia (A.b) und Trifolio arvensisFestucetalia ovinae (A.c) nachgewiesen.

In den Sandtrockenrasen der Unterklasse Koelerio-Corynephorenea kommen auf Saaremaa neben den an sandigen und instabilen Boden angepassten Arten (z. B. Carex arenaria), zahlreiche Trockenheit ertragende Kryptogamen vor (z. B. Tortula ruraliformis, Cetraria spp., Cladonia spp.).

\section{A.a - Corynephoretalia canescentis}

DENGLER (2004a) beschränkt sich in dieser Ordnung auf den Verband Corynephorion canescentis und schließt darin nur die gefäßpflanzenartenarmen Pionierstadien der Sandtrockenrasen ein. Im Gegensatz dazu vereinen POTT (1995) sowie SCHUBERT et al. (2001) drei Verbände in dieser Ordnung.

Im Rahmen dieser Diplomarbeit habe ich mich an den Gliederungsvorschlag von DENGLER (2004a) angelehnt, da dieser das Caricetum arenariae (s. u.) im Corynephorion einordnet.

\section{A1 - Caricetum arenariae}

Das Caricetum arenariae wird in der Literatur (z. B. PotT 1995, SCHUBERT et al. 2001, DENGLER 2004a) als Dominanzgesellschaft von Carex arenaria beschrieben. Abweichend zu der Einteilung von Dengler (2004a) führen PotT (1995) und SCHUBERT et al. (2001) die Gesellschaft im Verband Thero-Airion. Dies erachte ich als nicht sinnvoll, da es sich bei Carex arenaria und zahlreichen in dieser Gesellschaft vorkommenden Kryptogamen (z. B. Cladonia spp., Cetraria spp.) um ausdauernde Arten handelt, während die Gesellschaften des Thero-Airion vorwiegend durch anuelle oder kurzlebige Arten charakterisiert sind (DENGLER 2004a). 
Im Untersuchungsgebiet habe ich einige Bestände, in denen Carex arenaria zumeist als einzige Gefäßpflanzenart vorkommt, zum Caricetum arenariae gestellt (Tab. B).

Eine entsprechende Einheit wurde bislang in der estnischen Literatur nicht beschrieben.

Die Bestände des Caricetum arenariae aus dem Untersuchungsgebiet sind mit durchschnittlich 15 Arten auf $4 \mathrm{~m}^{2}$ relativ artenreich. Für Mecklenburg-Vorpommern werden 10 Arten pro $4 \mathrm{~m}^{2}$ angegeben (DENGLER 2004a: 307). Es stellt sich somit die Frage, ob die Zuordnung der auf Saaremaa vorkommenden Bestände zu dieser Assoziation sinnvoll ist. Eine Alternative wäre die Einordnung als ,gefäßpflanzenartenarme Ausbildung“ im Helichryso-Jasionetum, da die Bestände teilweise in unmittelbarem Kontakt $\mathrm{zu}$ diesem stehen und eine ähnlich zusammengesetzte Kryptogamenkombination aufweisen. Das Caricetum arenariae zeichnet sich jedoch häufig durch eine artenreiche Kryptogamenschicht aus (vgl. DENGLER 2001b), weshalb diese Trennung möglich ist.

\section{A.b - Sedo acris-Festucetalia}

Dengler (2004a) spaltet im Gegensatz zu Pott (1995) und Schubert et al. (2001) die Bestände der mesophilen Sandtrockenrasen von dieser Ordnung ab und ordnet sie in der Trifolio-Festucetalia ein.

Im Untersuchungsgebiet konnte ich den Zentralverband Koelerion glaucae mit den beiden Gesellschaften Helichryso-Jasionetum und Festucetum polesicae nachweisen.

\section{A2 - Helichryso arenarii-Jasionetum litoralis}

DENGLER (2004a) reiht die Assoziation nach einem europaweiten syntaxonomischen Vergleich im Koelerion glaucae ein, während sie SCHUBERT et al. (2001) zu den offenen Pionierrasen des Corynephorion canescentis stellen. Erstgenannter Autor stellt jene Bestände, die dem Festucetum polesicae aufgrund des Vorkommens von bezeichnenden Arten der Ordnung und Verbandes nahe stehen, aber durch das Fehlen von Festuca polesica und Koeleria glauca gekennzeichnet sind, zu dieser Assoziation.

Ich bin der Auffassung von DENGLER (2004a) gefolgt, da es sich bei den vorgefundenen Beständen zumeist nicht um frühe Pionierstadien der Sandtrockenrasen handelt. Hochstet vorkommende Arten wie Thymus serpyllum und einige Strauchflechten der Gattung Cladonia und Cetraria weisen eher auf ein späteres Sukzessionsstadium hin. Floristisch betrachtet ist das Helichryso-Jasionetum auf Saaremaa etwas verarmt. Die namensgebenden Arten Helichrysum arenarium (frühere Fundorte scheinen nach EICHWALD et al. 1978 mittlerweile erloschen zu sein) und Jasione montana kommen nicht bzw. zweitgenannte Art nur einmal in den Aufnahmen vor (Tab. C). Die Bestände entsprechen jedoch ansonsten floristisch jenen von DOLNIK (2003) von der Kurischen Nehrung und LÖBEL (2002) von Öland beschriebenen, weshalb ich sie dennoch dem Helichryso-Jasionetum zugeordnet habe.

Aus der estnischen Literatur lassen sich keine eindeutigen Entsprechungen $\mathrm{zu}$ dieser Assoziation ableiten.

Der mittlere pH-Wert der in beiden Fällen carbonatarmen Böden beträgt auf Saaremaa 6,7 und auf Öland 6,0 (LÖBEL 2002: 84). Auch Dengler (2004a) gibt das Vorkommen der Assoziation auf nicht zu sauren Sanden an. Nach ihm bevorzugt die Gesellschaft zudem skelettreiche Böden, was auf Saaremaa insbesondere für die Verrucaria spp.Potentilla tabernaemontani-Ausbildung (A2b) zutrifft (Tab. 21). Die Angaben der Autoren verdeutlichen die überregional ähnlichen Standortbedingungen der Assoziation. 


\section{A3 - Festucetum polesicae}

Festuca polesica und Koeleria glauca werden von verschiedenen Autoren (z. B. DIERßEN 1996, LÖBEL 2002, DENGLER 2001b, 2004a) als Charakterarten der Gesellschaft angegeben. Abweichend von der Klassifikation nach DENGLER (2004a) führt DIERßEN (1996) die Gesellschaft unter dem Namen Festuco-Koelerietum glaucae in der Ordnung Corynephoretalia auf. DENGLER (2004a) gibt für Mecklenburg-Vorpommern zusätzlich die im Anfangsstadium auf Cladonia (pyxidata agg.)-Schuppen schmarotzende Flechte Diploschistes muscorum als Charakterart an. Auf Saaremaa ist die Art in den ökologisch vergleichbaren Beständen des Festucetum polesicae und des Helichryso-Jasionetum zu finden. Das Vorkommen der Art ist ebenso in Mecklenburg-Vorpommern in beiden erwähnten Vegetationstypen zu erwarten, weshalb sie dort für das Festucetum polesicae meiner Meinung nach keine eindeutige Charakterart darstellt.

Am eigenen Aufnahmematerial lässt sich die Trennung des Helichryso-Jasionetum vom Festucetum polesicae deutlich durch eigene Charakterarten nachvollziehen, weshalb ich dem Vorschlag von DeNGLER (2004a) gefolgt bin. In den Beständen J14, X07 und X44 fehlt Festuca polesica (Tab. D). Laut DENGLER (2004a) ist es jedoch ohne weiteres möglich, Bestände ohne Festuca polesica, nur mit Koeleria glauca zum Festucetum polesicae zu stellen.

Für Estland erwähnt LiPPMAA (1933: 157) eine auf den Sandböden entlang der Küsten häufig vorkommende „Festuca polesica-Assoziation“. Von der Halbinsel Sõrve im Süden der Insel berichtet TOMSON (1937: 79) von einer Festuca polesica-Union. LAASIMER (1965: 94 f.) charakterisiert eine von Südost- bis Süd-Estland vorkommende „Koeleria glauca-Festuca polesica assotsiatioon“ und gibt ebenso wie TOMSON (1937: 79) Koeleria glauca, Festuca polesica, Dianthus arenarius, Thymus serpyllum, Galium verum u. a. als kennzeichnende Arten an. Stete Begleitarten sind nach ihm Kryptogamen wie Cladonia spp., Cetraria islandica, Polytrichum piliferum, Racomitrium canescens und Dicranum scoparium. Diese Artenkombination entspricht völlig den von mir auf Saaremaa vorgefundenen Beständen.

Bemerkenswerte Unterschiede $\mathrm{zu}$ anderen Gebieten Europas zeigen sich vor allem in der Bodenreaktion. Auf Saaremaa liegt der durchschnittliche $\mathrm{pH}-$ Wert des von der Gesellschaft besiedelten Bodens mit 7,4 deutlich höher als beispielsweise auf dem estnischen Festland (34) (LAASIMER 1965: 94), auf der Insel Öland (Ø 5,3) (LÖBEL 2002: 81) oder in Dänemark (4,6-5,3) (BÖCHER 1947 zit. n. LÖBEL 2002: 82). Ebenso liegen die Basensättigung und der Carbonatgehalt (Tab. 21) höher als die von LÖBEL (2002: 81) gemessenen Werte (auf Öland: Basensättignung 69,8\% bei carbonatfreiem Boden). DENGLER (1994: 242) weist auf eine breite Amplitude des pH-Wertes des Festucetum polesicae hin. Er unterscheidet eine basenarme Corynephorus- und eine basenreiche Thymus serpyllum ssp. serpyllum-Subassoziation. Im Untersuchungsgebiet fehlen basenarme Standorte, da sich die potenziellen Lebensräume der Gesellschaft dort auf die Dünengebiete in Küstennähe beschränken, welche aus noch relativ jungen marinen Sanden mit teilweise hohem Muschelkalkanteil bestehen. Gegenüber den anderen Regionen macht sich dieser standörtliche Unterschied in der Artenzusammensetzung durch ein Zurücktreten azidophytischer Sippen wie Hypochaeris radicata und das hochstete Vorkommen basiphytischer Moose (z. B. Tortella inclinata, Ditrichum flexicaule) bemerkbar (Tab. D).

\section{A.c - Trifolio arvensis-Festucetalia ovinae}

Die Ordnung Trifolio arvensis-Festucetalia ovinae umfasst nach DENGLER (2004a) Bestände, welche sich durch das Nebeneinander von Sandtrockenrasen und mesophileren Grünlandarten auszeichnen (Sand-Halbtrockenrasen). Er teilt die Ordnung in drei Verbände nach den (Haupt-)Verbreitungsgebieten verschiedener Festuca-Arten (F. filiformis, F. ovina und F. guestfalica, F. brevipila) ein. Aufgrund dieser Untergliederung gestalte sich die 
syntaxonomische Bear-beitung der Ordnung schwierig, da die biosystematische Bearbeitung der Schaf-Schwingel-Gruppe bislang noch nicht befriedigend abgeschlossen und zudem bestimmungskritisch sei. Das Synareal des Verbandes Armerion elongatae entspreche im Wesentlichen der Verbreitungsareale der Arten Armeria maritima ssp. elongata sowie Festuca brevipila. Des Weiteren gibt er den charakter- und differenzialartenfreien Zentralverband Hyperico perforati-Scleranthion perennis Moravec 1967 mit Festuca ovina an.

Die Einordnung der auf Saaremaa vorkommenden Bestände zu der Ordnung TrifolioFestucetalia ist nach dem Konzept von DENGLER (2004a) relativ unstrittig, da neben typischen Sandtrockenrasenarten wie Sedum acre und Cladonia furcata mesophilere Grünlandarten wie Achillea millefolium, Helictotrichon pratense und Plantago lanceolata hochstet vorkommen. Zudem haben die Ordnungscharakterart Peltigera canina sowie die Differenzialart Agrostis capillaris Mecklenburg-Vorpommerns (DENGLER 2001b) und Ölands (LöBEL 2002: Tabelle B) im Untersuchungsgebiet ihren Verbreitungsschwerpunkt in diesen Beständen (Tab. A), wodurch die Bindung an die Trifolio-Festucetalia unterstrichen wird. Auf Saaremaa kommt allerdings von den oben erwähnten Festuca-Arten nur Festuca ovina vor. Trotz des Fehlens der Verbandscharakterarten Armeria maritima ssp. elongata und Festuca brevipila im Untersuchungsgebiet habe ich mich dafür entschieden, die Bestände in den subkontinentalen Verband Armerion elongatae einzureihen, da mit Potentilla argentea agg., Elymus repens und Poa angustifolia Differenzialarten des Verbandes hochstet vorkommen (Tab. A). Dengler (2004a) gibt des Weiteren Vicia lathyroides als Charakterart sowie Potentilla argentea agg. als territoriale Charakterart von Mecklenburg-Vorpommern an. Letztgenannte wird in SCHUBERT et al. (2001) neben Trifolium arvense als hochstete Begleitart der Assoziation angegeben. Auf Saaremaa zeigen diese Arten ebenfalls einen Schwerpunkt in den Beständen (Tab. E), was diese Entscheidung stützt.

Somit habe ich im Untersuchungsgebiet den Verband Armerion elongatae nachgewiesen.

\section{A4 - Sileno otitae-Festucetum breviplilae}

Dengler (2004a) unterscheidet innerhalb des Verbandes das Diantho deltoidis-Armerietum elongatae Krausch ex Pötsch 1962 auf basenärmeren Standorten und das Sileno-Festucetum auf basenreicheren. Auch SchUBERT et al. (2001) nehmen diese Einteilung vor. DenGLER (2004a) weist darauf hin, dass es sich beim Sileno-Festucetum um die gleiche Gesellschaft wie das Pulsatillo pratensis-Phleetum phleoidis handelt, welche jedoch von einigen Autoren (z. B. Ротт 1995) fälschlicherweise als eigenständige Gesellschaften geführt wird zweitgenannte sogar in der Klasse Festuco-Brometea. Zudem erwähnt er die Zentralassoziation Thymo pulegioidis-Festucetum ovinae Oberd. 1957 des oben aufgeführten Zentralverbandes der Ordnung.

In Dengler (2004a) sind Acinos arvensis und Phleum phleoides Differenzialarten des SilenoFestucetum sowie Luzula campestris und Pimpinella saxifraga agg. ( $P$. saxifraga und $P$. nigra) gemeinsame Differenzialarten mit dem Diantho deltoidis-Armerietum elongatae Krausch ex Pötsch 1962. Das hochstete Vorkommen dieser Arten in den Beständen des Unter-suchungsgebietes (Tab. E) spricht somit für die Klassifikation im Armerion elongatae. Das Vorkommen von Acinos arvensis, Phleum phleoides, Helictotrichon pratense und weiterer basi- und mesophiler Sippen aus den Festuco-Brometea deutet auf die Zuordnung zum Sileno-Festucetum innerhalb des Verbandes hin. Die Klassifikation im ThymoFestucetum wäre jedoch bei einigen Beständen der Potentilla-Agrostis-Ausbildung (z. B. X03, X09; Tab. E) ebenso möglich. Die genaue Zugehörigkeit müsste deshalb anhand eines umfassenden überregionalen syntaxonomischen Vergleichs überprüft werden.

LIPPMAA (1933: 137 f.) berichtet von trockenen Abhängen aus dem Süden der Insel Manilaid, die „teilweise von einer eigenartigen Trockenwiese eingenommen“ werden. Er nennt die 
häufigsten dort vorkommenden Arten, welche eine Kombination aus Vertretern der Sand- und Halbtrockenrasen darstellen. Hierbei könnte es sich um das Sileno-Festucetum handeln. Eine eindeutige Aussage kann jedoch nicht getroffen werden, da der Autor die Bestände nicht durch Vegetationsaufnahmen dokumentiert hat.

Eine Thymus serpyllum-Galium verum-Union beschreibt TOMSON (1937: 29 f.) von der Halbinsel Sörve im Südwesten Saaremaas, welche neben Sandtrockenrasenarten mesophile Sippen der Festuco-Brometea aufweist. Die weiterhin aufgeführten Begleitarten lassen nach dem in dieser Arbeit verfolgten System eine Übereinstimmung mit dem Sileno-Festucetum vermuten.

LAASIMER (1965: 95 f.) beschreibt eine „Thymus serpyllum-Galium verum'i assotsiatsioon“ auf sandigen Böden der estnischen Küsten mit einigen mesophileren Sippen in ansonsten von Sandtrockenrasen dominierten Beständen, die hinsichtlich der von ihm beschriebenen Artenzusammensetzung vergleichbar mit jenen sind, die ich im Sileno-Festucetum eingeordnet habe. Der Autor hat jedoch keine Vegetationstabellen angefertigt, weshalb sich diese Entsprechung nur vermuten lässt.

Die Bestände auf Saaremaa entsprechen floristisch weitgehend jenen, die LöBEL (2002) von Öland beschrieben hat. LÖBEL (2002: 89) und FISCHER (2003:152) beschreiben die Standortverhältnisse der Gesellschaft von Öland bzw. des Elbtals als humus- und basenreiche Böden mit einer schwach sauren bis schwach basischen Bodenreaktion. Die dichte Vegetation und einige mesophile Arten lassen indes auf weniger kritische Verhältnisse als in den übrigen Ordnungen der Koelerio-Corynephorenea schließen, was mit den eigenen Ergebnissen übereinstimmt.

\subsubsection{Sedo-Scleranthenea}

Die Unterklasse Sedo-Scleranthenea wird nach Dengler (2004a) sowie DenGLER \& LöBEL (2006) in zwei Ordnungen unterteilt. Die Ordnung Sedo-Scleranthetalia umfasst demnach die azidophilen, die Ordnung Alysso alyssoidis-Sedetalia die basiphilen Felsgrusfluren. Betrachte man das Synareal der Unterklasse seien zwei voneinander getrennte Verbreitungsschwerpunkte zu erkennen: zum einen in den Gebirgsregionen Mitteleuropas und zum anderen von Südschweden in Richtung Nordosten über die großen Ostseeinseln bis Südfinnland (DENGLER 2003: 218, DENGLER \& LÖBEL 2006). DiERßEN (1996) fasst hingegen sämtliche Felsgrusgesellschaften Nordeuropas, KORNECK (1975) jene Mitteleuropas in der Ordnung SedoScleranthetalia zusammen.

In dieser Arbeit folge ich, wie bereits in Abschnitt 5.1.1 erläutert, dem Vorschlag von DENGLER (2004a).

Auf Saaremaa kommen auf den flachgründigen Kalkböden der Alvare, an Felsstandorten sowie auf fossilen Strandwällen ausschließlich basiphile Felsgrusfluren der Ordnung Alysso alyssoidis-Sedetalia vor. Teilweise werden Sekundärstandorte anthropogenen Ursprungs wie Industriebrachen, geschotterte Wegränder, Lesesteinhaufen oder übererdeter Beton besiedelt.

\section{B.a - Alysso alyssoidis-Sedetalia}

DENGLER \& LÖBEL (2006) spalten die Ordnung nach umfassendem soziologischen Vergleich in zwei geografisch voneinander getrennte Verbände auf: den in Mitteleuropa weit verbreiteten Zentralverband Alysso alyssoidis-Sedion Oberd. \& T. Müller in T. Müller 1961 und den nordischen Verband Tortello tortuosae-Sedion albi. Letztgenannter sei durch arktisch-alpine, mesophile, azidophile sowie Wechselfeuchte anzeigende Arten charakterisiert (vgl. Dengler \& Löbel 2006: Tab. 1). DIERßEN (1996) unterscheidet in Nordeuropa innerhalb der Ordnung die Verbände Alysso-Sedion sowie Veronico-Poion glaucae Nordh. 1943, wobei 
Letztgenannter in der alpinen Stufe bzw. der Arktis vorkomme. Der Autor stellt jedoch die synsystematische Stellung des Veronico-Poion in Frage (1. c.: 522 ff.).

DENGLER \& LÖBEL (2006) unterscheiden weiterhin innerhalb des Tortello tortuosae-Sedion albi zwei Unterverbände: den außerhalb der Alvare vorkommenden weitgehend negativ differenzierten Zentralunterverband Tortello tortuosae-Sedenion albi, in welchem sie zwei Assoziationen unterscheiden, sowie den „Alvar-Unterverband“ Tortello rigentisHelianthemenion oelandici mit vier Assoziationen. Letztgenannter ist einerseits durch Moose und Flechten, die zwar weit verbreitet sind, jedoch in Gesellschaften anderer Regionen deutlich seltener auftreten, sowie durch eine Reihe endemischer Sippen der großen schwedischen Inseln charakterisiert. Laut den Autoren ist das Verbreitungsareal des TortelloHelianthemenion somit auf die wenigen Regionen mit Alvargebieten wie Öland, Gotland, Västergötland (Kinnekulle) und West-Estland beschränkt.

Am eigenen Aufnahmematerial lässt sich die Zweiteilung des Tortello-Sedion in Unterverbände anhand eigener Charakter- bzw. Differenzialarten nachvollziehen (Tab. A), weshalb ich mich für diesen Vorschlag entschieden habe.

Auf Saaremaa habe ich folglich in der Ordnung Alysso alyssoidis-Sedetalia den Verband Tortello tortuosae-Sedion albi einschließlich seiner beiden Unterverbände Tortello tortuosaeSedenion albi und Tortello rigentis-Helianthemenion oelandici nachgewiesen.

\section{B1 - Cladonio symphycarpiae-Sedetum albi}

DENGLER \& LÖBEL (2006) unterscheiden im Unterverband Tortello tortuosae-Sedenion albi zwei Assoziationen: Das Ditricho flexicaulis-Sedetum acris Hallberg 1971, in dem Bestände zusammengefasst werden, die von HALLBERG (1971) in Bohuslän an der schwedischen Westküste auf schalenreichen Sandböden aufgenommen wurden, sowie die Zentralassoziation Cladonio symphycarpiae-Sedetum albi, in welche DENGLER \& LÖBEL (2006) sämtliche außerhalb der Alvare liegende Aufnahmen von Felsgrusfluren aus Nordeuropa stellen. Beide Gesellschaften seien überwiegend negativ differenziert und weisen ein Arteninventar auf, welches eine Bindung an kalkhaltige Substrate zeige. Die westschwedischen Bestände sind durch Bacidia bagliettoana, Tortella fragilis und Catapyrenium cinereum charakterisiert sowie durch mesophile Arten der Festuco-Brometea (Carex caryophyllea, Festuca rubra, Lotus cornuculatus, Luzula campestris, Pimpinella saxifraga, Poa pratensis agg., und Solidago virgaurea) oder durch Arten, welche in Küstennähe auftreten (Armeria maritima ssp. maritima, Taraxacum obliquum agg., Viola tricolor) differenziert. DIERßEN (1996: 617) berichtet, dass diese Bestände (er verwendet das Synonym Sedo-Tortelletum (Albertson 1946) Hallberg 1971) in Bohuslän „kleinflächig auf offenen, humusarmen Flecken in Halbtrockenrasen eingebettet" vorkommen. Es stellt sich somit die Frage, ob HALLBERG (1971) das Homogenitätskriterium (vgl. Abschnitt 3.1.1) eingehalten hat. Es könnte sein, dass es sich bei den dokumentierten Beständen um Übergänge zwischen Felsgrus- und Halbtrockenrasengesellschaften handelt, was die Häufung der differenzierenden Halbtrockenrasenarten erklären würde. Die oben aufgeführten Arten würden somit keine zweifelsfrei verwendbaren Differenzialarten darstellen, sondern möglicherweise nur einen scheinbaren Unterschied der Gesellschaften widerspiegeln. Um jedoch ein fundiertes Urteil darüber fällen zu können, müsste dies durch die Erstellung weiterer Vegetationsaufnahmen an der schwedischen Westküste sowie den Einschluss zusätzlicher Aufnahmen des schwedischen Festlands überprüft werden.

Die auf Saaremaa außerhalb der Alvare, auf anthropogenen Sekundärstandorten sowie auf Strandwällen vorkommenden Bestände habe ich demzufolge in der Assoziation Cladonio symphycarpiae-Sedetum albi zusammengefasst. 
Das Cladonio-Sedetum ist nach DENGLER \& LÖBEL (2006) wahrscheinlich die am weitesten verbreitete Gesellschaft des Tortello-Sedion. Das Areal erstreckt sich vom südlichen Teil Nordeuropas bis in den hohen Norden. Außerdem existieren nach Angaben der Autoren Vegetationsaufnahmen aus Oslofjord und Trondheimfjord (Norwegen) sowie den schwedischen Provinzen Uppland und Västergötland. Das Vorkommen der Assoziation auf Saaremaa fügt sich somit gut in das von DENGLER \& LÖBEL (2006) skizzierte Verbreitungsbild ein.

VILBERG (1927: 44 f., 132) beschreibt von den Alvaren Nord-Estlands ,die vollständig nackten Kalkfliesen“, welche aufgrund des Mangels an Substrat fast ausschließlich von den Moosen Ditrichum flexicaule, Encalypta vulgaris, Ceratodon purpureus, u. a. bewachsen sind. Zudem kommen jedoch die Therophyten Androsace septentrionalis, Erophila verna, Saxifraga tridactylitis und Arenaria serpyllifolia vor. Solche flachgründigen Alvare mit kompakten Gesteinsplatten, die sich unmittelbar an der Oberfläche befinden, kommen zwar auf Saaremaa nicht vor, stattdessen jedoch die zahlreichen Industriebrachen, ungenutzten Panzerstraßen oder Beton-Bunker, welche vergleichbare Habitate darstellen. Im Untersuchungsgebiet wächst an solchen Stellen häufig das Cladonio-Sedetum. Die von VILBERG (1927) beschriebene Artenkombination ist durchaus mit jener des Cladonio-Sedetum vergleichbar. Eine eindeutige Zuordnung ist jedoch nicht möglich, da in den Tabellen keine Kryptogamen aufgeführt sind und die intraspezifischen Sippen nicht getrennt wurden.

LiPPMAA (1933: 157) erwähnt eine „Sedum-Saxifraga-Cerastium-Assoziation“, die dem Cladonio-Sedetum entsprechen könnte. Hierbei handelt es sich jedoch nur um eine vage Vermutung.

PÄRTEL et al. (1999a) unterscheiden anhand einer Clusteranalyse mehrere Gesellschaften der estnischen Alvare. Die Bestände des „Cluster 5“ entsprechen floristisch wie auch standörtlich betrachtet dem Cladonio-Sedetum. Nach den Autoren kommen diese Bestände in den Küstenregionen Nordwestestlands sowie auf den Inseln Saaremaa und Hiiumaa auf Kalkschotter mit geringer Bodenauflage vor. Eine Kryptogamenbearbeitung fand nicht statt.

Das Cladonio-Sedetum ist hinsichtlich der Artenzusammensetzung dem Poo compressaeSaxifragetum tridactylitae Géhu 1961 ähnlich. Das Poo-Saxifragetum wächst größtenteils auf anthropogenen Standorten und stellt eine floristisch etwas verarmte Assoziation des in den deutschen (Mittel-) Gebirgen verbreiteten Alysso alyssoidis-Sedion dar. Es ist ebenso überwiegend negativ gekennzeichnet, wie etwa durch das Fehlen von Sedum album (vgl. DENGLER 2004a). Es ist dennoch sinnvoll, die beiden Assoziationen in zwei unterschiedliche Verbände zu trennen, da sich der Alysso-Sedion und der Tortello-Sedion deutlich durch eine Reihe von Charakter- und Differenzialarten abgrenzen (vgl. DENGLER \& LöBEL 2006: Tab. 1). Das von JERMACĀNE \& LAIVIN̦Š (2001) aus Lettland beschriebene Saxifrago tridactylitoPoetum compressae (Kreh 1945) Géhu et Leriq 1957 entspricht folglich dem CladonioSedetum und sollte deshalb nicht, wie von den Autoren vorgeschlagen, im Alysso-Sedion eingereiht werden.

\section{B2 - Crepido pumilae-Allietum alvarensis}

DENGLER \& LÖBEL (2006) unterscheiden innerhalb des Tortello-Helianthemenion vier Assoziationen, die in ihrem Vorkommen auf die nordischen Alvargebiete beschränkt sind. Laut Autoren differieren die Standorteigenschaften der Assoziationen deutlich. Das CrepidoAllietum sowie das Fulgensio bracteatae-Poetum alpinae (Albertson 1950) Dengler \& Löbel 2006 kommen auf im Winter regelmäßig überfluteten Flächen vor, wobei erste Assoziation auf tiefgründigeren, durch Frostwechselerscheinungen stark beeinflussten Böden und zweite auf den flachgründigsten Böden mit der höchsten Deckung offenliegender Gesteine wachse. Das Helianthemo oelandici-Galietum oelandici Krahulec et al. ex Dengler \& Löbel 2006 sowie Gypsophilo fastigiatae-Globularietum vulgaris Krahulec et al. ex Dengler \& Löbel 
2006 komme hingegen auf etwas tiefgründigeren, weniger überfluteten und stabileren Böden vor. Das Crepido-Allietum ist nach den Autoren durch Crepis tectorum ssp. pumila und Artemisia rupestris charakterisiert sowie durch eine Gruppe Wechselfeuchte anzeigender Sippen differenziert (DENGLER \& LÖBEL 2006: Tab. 1). Das Fulgensio-Poetum zeichnet sich demnach floristisch hauptsächlich durch eine Reihe epigäischer Krustenflechten sowie das hochstete Vorkommen von Poa alpina aus. Das Helianthemo-Galietum ist schwach durch Galium oelandicum und Sisymbrium supinum charakterisiert, während die Kryptogamenschicht aus einer Reihe von Strauchflechten der Gattungen Cladonia und Cetraria aufgebaut ist. Das Gypsophilo-Globularietum ist durch Globularia vulgaris, Gypsophila fastigiata und Arabis hirsuta var. glaberrima charakterisiert. Es differenziert sich durch weitere Arten mit einer südöstlichen Verbreitung vom Helianthemo-Galietum. Außerdem fehlen die Wechselfeuchtezeiger.

DIERßEN (1996: Tab. 84) unterscheidet ebenfalls sechs Gesellschaften, jedoch sämtliche innerhalb der Ordnung Alysso-Sedetalia. Die Unterscheidung der Assoziationen wird in ähnlicher Weise wie bei DENGLER \& LÖBEL (2006) vollzogen. Letztgenannte konnten allerdings eine Vielzahl von Charakterarten hinzufügen.

Die Vegetation der Alvare Saaremaas weist - neben einigen lokalen Unterschieden in der Artenzusammensetzung - viele Gemeinsamkeiten mit jener der öländischen auf, weshalb die Klassifikation der aufgenommenen Bestände im Tortello rigentis-Helianthemenion oelandici gerechtfertigt ist. Beispielsweise kommen auch auf Saaremaa verschiedene Wechselfeuchtezeiger vor und differenzieren gegen das Tortello-Sedenion (Tab. A). Die geringsten Gemeinsamkeiten der Bestände auf Saaremaa sind mit dem Fulgensio-Poetum sowie dem Gypsophilo-Globularietum festzustellen. Floristische Übereinstimmungen der auf Saaremaa von mir dokumentierten Bestände sind insbesondere mit dem Crepido-Allietum zu erkennen. In den entsprechenden Beständen auf Saaremaa kommt eine morphologisch Crepis tectorum ssp. pumila gleichende Form von Crepis tectorum vor (Abschnitt 5.3), Artemisia rupestris ist hingegen deutlich häufiger zu finden und wächst in $63 \%$ der aufgenommenen Bestände. Auch die vorkommenden Wechselfeuchtezeiger verdeutlichen die floristische Nähe und sprechen für die Klassifikation im Crepido-Allietum (Tab. G).

Gemeinsamkeiten in der Artenzusammensetzung sind ebenfalls zwischen einigen Beständen der Cetraria-Echium-Ausbildung (Abschnitt 4.1.6) und jenen des Helianthemo oelandiciGalietum oelandici Krahulec et al. ex Dengler \& Löbel 2006 zu erkennen (vgl. DENGLER \& LÖBEL 2006). Einige Differenzialarten des Helianthemo-Galietum wie Artemisia campestris, Cetraria aculeata, C. islandica und Potentilla tabernaemontani kommen vor, es fehlen jedoch die Charakterarten der Gesellschaft. Die Assoziation ist allerdings von DENGLER \& LÖBEL (2006) als Zentralassoziation konzipiert, welcher auch auf Öland in den meisten Beständen die Charakterarten fehlen. Die vorhandene Artenkombination legt die Zuordnung zum Crepido-Allietum größtenteils näher. DENGLER \& LÖBEL (2006) führen zudem Nostoc spec. als abgrenzende Differenzialart des Crepido-Allietum gegenüber dem HelianthemoGalietum an. Diese Art kommt in einem Großteil der Bestände auf Saaremaa vor. Eine eindeutige Trennung der beiden Gesellschaften ist im Untersuchungsgebiet in einigen Fällen dennoch nicht möglich. Durch einen umfassenderen syntaxonomischen Vergleich mit den vorliegenden Daten von DENGLER \& LÖBEL (2006) könnte diese Unklarheit in einer weiterführenden Studie beseitigt werden.

VILBERG (1927: 70 ff.) dokumentiert von nordestnischen Alvaren Bestände mit hochstetem Vorkommen von Allium schoenoprasum, Crepis tectorum und Sagina nodosa, deren sonstige Artenkombination auf die Zugehörigkeit zum Crepido-Allietum schließen lässt. Eine eindeutige Zuordnung ist jedoch nicht möglich, da in den Tabellen keine Kryptogamen aufgeführt sind und die intraspezifischen Sippen nicht getrennt wurden. 
LIPPMAA (1933: 157) teilt die Vegetation der Alvare in „Pflanzenvereine“ ein. Diese Beschreibung von Synusien ist jedoch nicht kompatibel mit der Klassifikation von Gesellschaften und deshalb unbrauchbar für einen Vergleich in dieser Arbeit. Eine „Thymus serpyllum-Galium verum-Ditrichum flexicaule-Assoziation“ erwähnt LAASIMER (1965: 146 f.). Er gibt zusätzlich eine Sedum acre-Sedum album-Variante auf exponierten Kalkgesteinen und eine Anthyllis vulneraria-Variante an.

ZOBEL (1987: 28 ff.) vergleicht Bestände von Alvaren Westestlands einschließlich der großen Inseln hinsichtlich der floristischen Komposition sowie ihres des Lebensraumes und unterscheidet drei Hauptgruppen. Gruppe $C$ repräsentiert Bestände auf flachgründigen Böden, welche durch Frostwechselerscheinungen beeinflusst sind. Er vergleicht diese mit dem Sedetum alvarense von ALBERTSON (1950). Festuca ovina und Sedum album kommen konstant vor. Er unterscheidet zusätzlich die Untergruppen $\mathrm{C} 1$ und $\mathrm{C} 2$, wobei letztere die „typischen Alvargesellschaften“ über kompaktem Kalkgestein darstellt. Diese Untergruppe ist durch Sedum album, einige Moosarten wie Tortella tortuosa und Ditrichum flexicaule sowie Flechtenarten wie Cladonia symphycarpia und C. chlorophaea charakterisiert. Bei letztgenannter Art scheint es sich jedoch um einen Bestimmungsfehler zu handeln, da Cladonia chlorophaea auf sauren Substraten wächst. Auf den von ZoBEL (1987) untersuchten basischen Substraten kommt die ebenfalls bechertragende Art Cladonia pocillum vor. Laut ihm entspricht diese Gesellschaft dem von ALBERTSON (1950) beschriebenen Sedetum alvarense. Die erste Gesellschaft stellt einen Übergang zu Gruppe B, den Halbtrockenrasen, dar. Er klassifiziert diese Untergruppe als eine Untereinheit des Sedetum alvarense mit stetem Vorkommen von Festuca ovina, Artemisia rupestris und A. campestris. Der von ihm erstellten Tabelle ist $\mathrm{zu}$ entnehmen, dass außerdem Wechselfeuchtezeiger wie Agrostis stolonifera und mesophile Sippen wie Helictotrichon pratense häufige Begleitarten sind. Eine eindeutige Trennung der Gruppen wird aus seiner Tabelle jedoch nicht ersichtlich. Der Autor verbalisiert zwar das Vorkommen einiger Kryptogamen im Text (s. o.), in der Vegetationstabelle fehlen diese jedoch. Der größte Anteil der Charakter- und Differenzialarten innerhalb des Tortello-Helianthemenion wird in dieser Arbeit sowie in DENGLER \& LÖBEL (2006) von Kryptogamen gestellt. Eine Unterscheidung von Assoziationen nach dem in dieser Arbeit verwendeten Konzept ist somit nicht möglich. Die Klassifikation sämtlicher von ZOBEL (1987) dokumentierten Bestände im Crepido-Allietum ist jedoch hinsichtlich der Gefäßpflanzen-artenkombination durchaus denkbar. Die Gruppe $\mathrm{C} 1$ ist beispielsweise reich an Wechsel-feuchtezeigern und könnte somit der Artemisia rupestris-Ausbildung (Abschnitt 4.1.6; Tab. G) entsprechen.

PÄRTEL et al. (1999a) fassen in Cluster 6 auf Saaremaa und Hiiumaa vorkommende Bestände auf flachgründigen Böden mit Frosteinfluss zusammen. Die Arten Artemisia campestris, A. rupestris, Acinos arvensis und Sedum album kommen neben wechselfeuchte Verhältnisse anzeigende Arten hochstet vor (vgl. 1. c.: Tab. 1). Diese Bestände entsprechen jenen der in dieser Arbeit im Crepido-Allietum eingeordneten. Eine Kryptogamenbearbeitung durch die Autoren fand allerdings nicht statt.

Ökologisch betrachtet sind nur geringe Unterschiede zwischen den Standorten auf Saaremaa und Öland festzustellen. DeNGLER \& LÖBEL (2006) trennen zusätzlich eine Festuca oelandica-Ausbildung ab. Sie ist extrem stark durch Frosteffekte gestört und weist deshalb sehr geringe Artenzahlen auf. Ein entsprechendes Pendant fehlt auf Saaremaa.

\subsubsection{Festuco-Brometea}

Die syntaxonomische Einteilung dieser Klasse wird von verschiedenen Autoren kontrovers diskutiert. Die gängigste Variante ist die Unterteilung nach pflanzengeografischen Verbreitungsmustern entlang eines Kontinentalitätsgefälles in vikariierende Ordnungen. Eine überregional befriedigende Unterteilung für Europa liegt zurzeit jedoch nicht vor. 
Nach Dengler (2004b) werden in der Klasse Festuco-Brometea die basiphilen Halbtrockenrasen und Steppen zusammengefasst.

Einige Autoren (z. B. PотT 1995: 349 ff.) teilen die Klasse Festuco-Brometea in zwei Ordnungen ein: die Brometalia erecti mit submediterran-subatlantischer Verbreitung sowie die Festucetalia valesiacae mit kontinentaler Verbreitung. Innerhalb der Ordnungen werden jeweils ein meso- und ein xerophiler Verband unterschieden. DENGLER (2004b: 327) führt an, dass dadurch in Mitteleuropa floristisch verwandte Halbtrockenrasen in unterschiedlichen Ordnungen zusammengeführt werden. Vergleiche man die unterschiedlichen mesophilen Gesellschaften miteinander, fiele auf, dass etliche Halbtrockenrasenarten (z. B. Filipendula vulgaris, Helictotrichon pratense, Homalothecium lutescens) in sämtlichen Beständen konstant auftreten und als Charakterarten angesehen werden können. Dies macht eine sinnvolle Trennung kaum möglich. KORNECK (1974) schlägt deshalb vor, diese verwandten mesophilen Halbtrockenrasen in der Ordnung Brachypodietalia pinnati (= Brometalia erecti) zusammenzufassen. Die xerophilen Gesellschaften unterscheidet er wiederum in zwei Ordnungen mit einem westlichen und einem östlichen Verbreitungsschwerpunkt, welche er den mesophilen Halbtrockenrasen gegenüberstellt.

Auf Saaremaa kommen in diesen Beständen eine Reihe mesophiler Sippen vor (Tab. A), die nach Dengler (2004b) Ordnungscharakter- bzw. Differenzialarten der Brachypodietalia pinnati darstellen, was für die Klassifikation in dieser Ordnung spricht.

Ich habe auf Saaremaa somit die Ordnung Brachypodietalia pinnati nachgewiesen.

\section{C.a - Brachypodietalia pinnati}

DENGLER (2004b: 329 f.) unterscheidet in den wärmebegünstigten Regionen Europas innerhalb der Brachypodietalia drei Verbände: das Bromion erecti W. Koch 1926 mit einer Verbreitung in Westeuropa und im westlichen Mitteleuropa, das Cirsio-Brachypodion pinnati Hadač \& Klika in Klika \& Hadač 1944 mit einer Verbreitung in den subkontinentalen Bereichen Zentraleuropas und das in Osteuropa vorkommende Agrostio vinealis-Avenulion schellianae Royer 1991. DENGLER \& LöBEL (in DENGLER et al. 2003) weisen zusätzlich den Zentralverband Filipendulo vulgaris-Helictotrichion pratensis Dengler \& Löbel in Dengler et al. 2003 mit einem Verbreitungsschwerpunkt im nördlichen Europa aus.

Die basiphilen Halbtrockenrasen auf Saaremaa sind floristisch recht einheitlich. Anhand des vorliegenden Aufnahmematerials lassen sich die Bestände nicht sinnvoll zu einem der beiden Verbände zuordnen. Eine umfassende synthetische Studie der Kalk-Halbtrockenrasen im skandinavischen und baltischen Raum befindet sich deshalb in Vorbereitung (DENGLER et al. i. V.).

Aufgrund der unklaren Stellung im syntaxonomischen System führe ich die auf Saaremaa vorkommenden Bestände informell als Helictotrichon pratense-[Brachypodietalia pinnati]Gesellschaft.

\section{C1 - Helictotrichon pratense-[Brachypodietalia]-Gesellschaft}

Die Gesellschaft ist im Untersuchungsgebiet durch eine Reihe territoriale Charakterarten gekennzeichnet (Tab. A).

In der estnischen Literatur werden zahlreiche Angaben zu Beständen mit unterschiedlichsten Klassifikationen angegeben. Ein umfassendes Gesamtkonzept ist jedoch nicht erkennbar.

LIPPMAA (1933: 157) beschreibt eine von Helictotrichon pratense dominierte Filipendula vulgaris-Trifolium montanum-Synusie, die in Estland auf kalkreichem Untergrund weit verbreitet ist. Die angegebene Artenkombination stimmt mit jener der auf Saaremaa vorkommenden Artenkombination der Halbtrockenrasen überein. 
LAASIMER (1965: 147) erwähnt Ebendiese und unterscheidet zusätzlich eine außerhalb der Alvare vorkommende „Filipendula hexapetala-Sesleria coerulea-Carex montana'i assotsiatsioon" (1. c.: 150 f.), welche hinsichtlich der Artenzusammensetzung einige Gemeinsamkeiten $\mathrm{zu}$ der „Filipendula hexapetala-Trifolium montanum'i assotsiatsioon“ aufweist, jedoch auf etwas bessere Nährstoff- und Wasserverhältnisse hindeutet.

ZoBEL (1987) schlägt nach eingehendem Vergleich syntaxonomischer Studien, die von verschiedenen Autoren über die estnische Alvar-Vegetation erstellt wurden, den Namen Helictotrichon pratense-Gesellschaft für die Halbtrockenrasen vor. Anhand eigener Aufnahmen fasst er Bestände, in welchen neben den konstant auftretenden Arten wie Helictotrichon pratense, Anthyllis vulneraria und Carex caryophyllea außerdem Sesleria caerulea, Galium verum, Thymus serpyllum und Festuca ovina auf den weniger flachgründigen Alvarböden vorkommen, in Gruppe B zusammen (1. c.: 38 ff.). Als konstant auftretende Moosarten nennt er Homalothecium lutescens, Hypnum cupressiforme var. lacunosum und Dicranum scoparium. Des Weiteren nimmt er eine Unterteilung vor, welche sich allerdings anhand der Vegetationstabelle aufgrund der ähnlichen Artenzusammensetzung nicht sinnvoll nachvollziehen lässt. Ausschließlich die Gruppe B2a, eine nach ZOBEL (1987) auf kiesreichem Substrat wachsende Ausbildung, welche von Calluna vulgaris dominiert ist, lässt sich aufgrund der Artenkomposition trennen.

Im eigenen Aufnahmematerial sind hinsichtlich der Artenzusammensetzung (mit Ausnahme von B2a) überwiegend Gemeinsamkeiten mit den Angaben von ZoBEL (1987) festzustellen. Dicranum scoparium kann jedoch im Untersuchungsgebiet nicht als steter Begleiter der Halbtrockenrasen gezählt werden.

PÄRTEL et al. (1999a) beschreiben unterschiedliche Ausbildungen des „Avenetum alvarense“ aus Westestland sowie den westlichen estnischen Inseln. In den Clustern 2, 3 und 4 werden Halbtrockenrasen zusammengefasst. Von der Insel Saaremaa stammen Vegetationsaufnahmen aus sämtlichen erwähnten Ausbildungen. Die Artenzusammensetzungen der unterschiedenen Cluster weichen allerdings nur geringfügig voneinander ab. Die eigenen Aufnahmen stimmen weitgehend mit jenen von PÄRTEL et al. (1999a) überein.

Aus Lettland beschreibt RUSIN̦A (2005) Bestände, welche den Halbtrockenrasen auf Saaremaa morphologisch sehr ähnlich sind. Charakterarten sind dort neben den dominanten Vertretern Helictotrichon pratense und Filipendula vulgaris noch Fragaria viridis, Pimpinella saxifraga, Galium verum, Centaurea scabiosa, Briza media und Trifolium montanum sowie einige Arten der Molinio-Arrhenatheretea wie Festuca rubra, Plantago lanceolata, Knautia arvensis und Achillea millefolium. Die Autorin ordnet die Bestände als Basalassoziation in der Ordnung Brometalia erecti ein.

Vergleicht man die Aufnahmen von Saaremaa mit jenen Ölands, fällt eine geringe Übereinstimmung mit dem vorrangig auf dem Großen Alvar verbreiteten Veronico spicataeAvenetum Krahulec et al. 1986 (vgl. LöBEL 2002: Tabelle C) auf. Deutlich besser entsprechen die Aufnahmen der von LÖBEL (2002) außerhalb des öländischen Großen Alvars beschrie-benen Trifolium montanum-Medicago falcata-Gesellschaft.

\subsubsection{Zusammenfassung der syntaxonomischen Klassifikation}

Insgesamt konnte ich auf der Insel Saaremaa sieben Trockenrasengesellschaften auf Assoziationsebene unterscheiden. Sechs davon ließen sich bekannten Assoziationen mit formal gültigen Syntaxonnamen zuordnen. Die Stellung der siebten Trockenrasengesellschaft ist bislang noch nicht geklärt, weshalb ich sie einstweilen auf informeller Ebene aufführe. Die Assoziationen verteilen sich im System von Dengler (2004a, 2004b) sowie DenGler \& LÖBEL (2006) auf vier Verbände, fünf Ordnungen, und zwei Klassen (Tab. 40). Innerhalb der Unterklasse Koelerio-Corynephorenena gab es nur eine gänzlich adäquate, eine weniger 
adäquate sowie in zwei Fällen keine Übereinstimmung mit der bisherigen estnischen Klassifikation. Hinsichtlich der Sedo-Scleranthenea- und Festuco-Brometea-Gesellschaften konnte ich hingegen zutreffende Parallelen herleiten.

Tab. 40: Syntaxonomische Übersicht der im Untersuchungsgebiet nachgewiesenen Trockenrasengesellschaften. Zentralsyntaxa sind mit einem Sternchen $(*)$ gekennzeichnet.

AB Koelerio-Corynephoretea Klika in Klika \& V. Novák 1941

A Koelerio-Corynephorenea (Klika in Klika \& V. Novák 1941) Dengler in Dengler et al. 2003

A.a Corynephoretalia canascentis Klika 1934 *

A.a1 Corynephorion canescentis Klika 1931

A1 Caricetum arenariae Christiansen 1927 *

A.b Sedo acris-Festucetalia Tx. 1951

A.b1 Koelerion glaucae Volk 1931

A2 Helichryso arenarii-Jasionetum litoralis Libbert 1940 *

A3 Festucetum polesicae Regel 1928

A.c Trifolio arvensis-Festucetalia ovinae Moravec 1967

A.c1 Armerion elongatae Pötsch 1962

A4 Sileno otitae-Festucetum brevipilae Libbert 1933 corr. Kratzert \& Dengler 1999

B Sedo-Scleranthenea (Br.-Bl. 1955) Dengler in Dengler et al. 2003

B.a Alysso alyssoidis-Sedetalia Moravec 1967

B.a1 Tortello tortuosae-Sedion albi Hallberg ex Dengler \& Löbel 2006

B.a1a Tortello tortuosae-Sedenion albi (Hallberg ex Dengler \& Löbel) Dengler \& Löbel 2006 *

B1 Cladonio symphycarpiae-Sedetum albi Tx. $1951^{3}$

B.a1b Tortello rigentis-Helianthemion oelandici suball. nov. Dengler \& Löbel 2006

B2 Crepido pumilae-Allietum alvarensis Krahulec et al. ex Dengler \& Löbel 2006

C Festuco-Brometea Br.-BI. \& Tx. ex Klika \& Hadač 1944

C.a Brachypodietalia pinnati Korneck 1974

C1 Helictotrichon pratense-[Brachypodietalia pinnati]-Gesellschaft

\subsection{FFH-Schutzstatus der Trockenrasengesellschaften des Untersuchungsgebietes}

In Anhang I der Flora-Fauna-Habitat-Richtlinie der EU werden „natürliche Lebensraumtypen von gemeinschaftlichem Interesse, für deren Erhaltung besondere Schutzgebiete ausgewiesen werden müssen“ (EUROPEAN COMMISSION 2003a) aufgeführt sowie im Interpretationshandbuch der Lebensräume der Europäischen Union (EUROPEAN COMMISSION 2003b) charakterisiert. Im Folgenden werden die mit den jeweiligen Gesellschaften oder deren Untereinheiten ganz oder teilweise übereinstimmenden Lebensraumtypen angegeben. Zudem werden die in EUROPEAN COMMISSION (2003b) aufgeführten typischen Arten der geschützten Lebensraumtypen, welche in den Trockenrasen Saaremaas vorkommen, aufgezählt. Prioritär geschützte Lebensraumtypen sind mit einem Sternchen (*) gekennzeichnet. Der für die Umsetzung der FFH-Richtlinie erforderliche Schutzstatus zur Erhaltung dieser Lebensräume wird auf nationaler Ebene festgelegt.

\footnotetext{
${ }^{3}$ Die von Dengler \& LöBel (2006) differierende Schreibweise begründet sich durch die geänderte Nomenklaturregelung bezüglich Cladonia symphycarpia in SANTESSON et al. (2004).
} 


\section{*2130 - Festliegende Küstendünen mit krautiger Vegetation (Graudünen) (Nordic classification: ,4142 - Festuca rubra-Hieracium umbellatum-typ“)}

Typische in den Trockenrasen der Dünen Saaremaas vorkommende Arten sind Bromus hordeaceus, Carex arenaria, Cerastium spp., Cladonia spp., Galium verum, Koeleria spp., Myosotis ramosissima, Tortula ruraliformis und Viola rupestris.

Die strauchflechtenreiche Ausbildung des Caricetum arenariae, sämtliche auf den küstennahen Dünen wachsende Bestände des Helichryso-Jasionetum sowie des Festucetum polesicae könnten somit diesem Lebensraumtypen zugeordnet werden.

\section{*6110 - Lückige basiphile oder Kalk-Pionierrasen (Alysso-Sedion albi)}

Bestände des Cladonio-Sedetum auf natürlichen Standorten könnten diesem Lebensraumtypen zugeteilt werden. Als typische, auf Saaremaa vorkommende Arten werden Cerastium spp., Hornungia petraea, Saxifraga tridactylites und Sedum spp. angegeben.

\section{*6120 - Trockene, kalkreiche Sandrasen (Nordic classification: „5141 - Koeleria glauca- typ")}

Auf Saaremaa kommen die Arten Alyssum montanum ssp. gemelinii, Dianthus deltoides, Gypsophila fastigiata, Herniaria glabra und Koeleria glauca in einigen Sandtrockenrasenbeständen vor. Insbesondere die Bestände der Koeleria-Ausbildung sowie mit Einschränkungen der Placynthiella-Cladonia-Ausbildung (mit Gypsophila fastigiata) des Festucetum polesicae könnten deshalb ebenso zu diesem Lebensraumtypen gezählt werden.

\section{0 - Naturnahe Kalk-Trockenrasen und deren Verbuschungsstadien (Festuco-}

\section{Brometalia)}

Im Untersuchungsgebiet vorkommende Bestände der Helictotrichon-Gesellschaft, in denen Arten wie Anthyllis vulneraria, Arabis hirsuta, Campanula glomerata, Carex caryophyllea, Carlina vulgaris, Centaurea scabiosa, Leontodon hispidus, Medicago sativa ssp. falcata, Polygala comosa, Primula veris, und Scabiosa columbaria vorkommen, könnten diesem Lebensraumtypen untergeordnet werden. Potenziell möglich, jedoch von mir nicht dokumentiert, wäre das Vorkommen von Ophrys insectifera, Orchis mascula, O. militaris, $O$. morio, O. purpurea, O. ustulata. Bestände, in welchen diese Orchideen anwesend sind, werden als prioritär eingestuft.

*6270 - Artenreiche, mesophile, trockene Rasen der niederen Lagen Fennoskandiens (Nordic classification: ,5212 - Festuca ovina-Lychnis viscaria-type; 5213 - Avenula pratensis-Fragaria viridis-Filipendula vulgaris-type“")

Aufgrund des mesophilen Charakters und der Artenkombination wäre die Zuordnung einiger Bestände des Sileno-Festucetum sowie der Helictotrichon-Gesellschaft zu diesem prioritär geschützten Lebensraumtyp durchaus möglich. Typische Arten, welche auf Saaremaa vorkommen, sind Agrostis capillaris, Alchemilla spp., Antennaria dioica, Botrychium spp., Dianthus deltoides, Euphrasia spp., Festuca ovina, F. rubra, Galium verum, Gymnademia conopsea, Hypochoeris maculata, Leontodon hispidus, Lychnis viscaria, Plantago lanceolata, Primula veris und Ranunculus polyanthemos. 
*6280 - Nordische Alvar-Trockenrasen und flache praekambrische Kalkfelsen (Nordic vegetation types: ,5151 - Sedum album-Tortella spp.-type, 5152 - Festuca ovina-Tortella spp.-type ${ }^{6}$ )

Sämtliche auf Alvaren vorkommende Bestände des Crepido-Allietum gehören diesem prioritär geschützten Vegetationstyp an. Auf Saaremaa kommen Allium schoenoprasum var. alvarense, Androsace septentrionalis, Asperula tinctoria, Aspicilia calcarea, Cladonia pocillum, C. symphycarpia, Crepis tectorum ssp. pumila, Ditrichum flexicaule, Encalypta spp., Festuca ovina, Flavocetraria nivalis, Fulgensia spp., Hornungia petraea, Inula salicina, Linum catharticum, Potentilla tabernaemontani, Saxifraga tridactylites, Schistidium apocarpum, Sedum acre, S. album und Tortella tortuosa und somit eine große Anzahl der in EUROPEAN COMMISSION (2003b) aufgefürten Sippen vor.

\subsection{Besonderheiten der Flora}

Im Folgenden werden die Funde neuer und interessanter Gefäßpflanzen-, Moos- und Flechtenarten für Estland diskutiert.

\section{Gefäßpflanzen}

\section{Allium schoenoprasum L.}

Allium schoenoprasum ssp. schoenoprasum var. alvarense Hyl. wird in LUNDQVIST (1986) als endemisch für Öland, in JONSELL \& KARLSSON (2004: 158) für Öland und Gotland angegeben. Nach EICHWALD et al. (1984) kommt die Pflanze jedoch selten in den Alvargebieten auf Saaremaa und im Norden Estlands vor. Sie habe eine geringere Wuchshöhe als die Nominat-Varietät (LundQvist 1986, Mossberg \& STENBERG 2003; 7-15 cm werden in EICHWALD et al. 1984 angegeben), eine blau-weiß bereifte Kutikula (EICHWALD et al. 1984, Mossberg \& Stenberg 2003) und kürzere Blätter als der den Blütenstand tragende Stängel (EICHWALD et al. 1984), welche immer aufrecht wachsen (MOSSBERG \& STENBERG 2003).

Die von mir auf den Alvarflächen Saaremaas gefundenen Allium schoenoprasumPopulationen entsprachen völlig dieser Diagnose von var. alvarense und werden deshalb in meiner Arbeit unter diesem Namen geführt. Auf Saaremaa kommen sie auf den Alvarflächen im westlichen Teil der Insel sowie bei Iipla vor und besiedeln dort vorwiegend wechselfeuchte Stellen, was der Standortangabe von RosÉN \& BORGEGÅRD (1999: 128) entspricht. Allium schoenoprasum var. alvarense ist mit einer Stetigkeit von $20 \%$ eine territoriale Charakterart des Crepido-Allietum (Abschnitt 4.1.6; Tab. A, Tab. G).

\section{Crepis tectorum L.}

Die taxonomische Einordnung dieser Sippe scheint bislang noch nicht abschließend geklärt zu sein, weshalb die Verbreitungsangaben in der Literatur differieren. Crepis tectorum ssp. pumila kommt nach MosSBERG \& STENBERG (2003), JONSELL \& KARLSSON (2004: 150) bzw. ANDERSSON (1990) endemisch auf den kalkhaltigen Böden Ölands vor, nach TUTIN et al. (1976) auch auf Gotland. LEHT (1999) gibt einen Fundort der Sippe auf einem Alvar in Nordwestestland an. ANDERSSON (1990) erwähnt hingegen eine „outcrop-form” von Crepis tectorum, welche auf dem estnischen Festland sowie auf den westlichen Inseln vorkommt und sich phänotypisch von der ,weed“-Form (ssp. tectorum) durch die geringere Höhe, die weniger zerschlitzten Blätter und die kleineren Köpfe unterscheidet. Laut LUNDQVIST (1986) ist Crepis tectorum ssp. pumila kleinwüchsig, stark behaart, hat kleine Blütenköpfe und tritt auf den steinigen Böden auf Öland häufig in Massen auf. Nach TUTIN et al. (1976) sowie Mossberg \& Stenberg (2003) erreicht die Pflanze eine Höhe von bis $\mathrm{zu} 7 \mathrm{~cm}$, hat 2-4 
Stängelblätter und bildet 2-7 Köpfchen aus. Laut erstgenannten Autoren hat er zahlreiche Grundblätter und ungleiche Drüsenhaare. Nach zweitgenannten Autoren ist die Pflanze grauhaarig und verzweigt sich bereits kurz über der Basis.

Die von mir auf den Alvarflächen Saaremaas gesammelten Pflanzen wiesen eine Wuchshöhe von 3-11 cm auf, hatten 1-2 cm lange lanzettliche, unzerteilte und schwach gezähnte Grundsowie 1-4 etwa 1-1,5 cm lange linealische Stängelblätter. Sie bildeten 1-3 Köpfe, mit 5$9 \mathrm{~mm}$ langen Hüllblättern sowie einem Durchmesser von $0,7-1,5 \mathrm{~cm}$ aus. Neben zahlreichen Drüsenhaaren waren einfache Haare vorhanden. Morphologisch gleichen diese Pflanzen somit Crepis tectorum ssp. pumila. Auf Saaremaa ist die Sippe in einigen Alvargebieten zu finden und stellt mit einer Stetigkeit von $17 \%$ eine territoriale Charakterart des CrepidoAllietum dar (Abschnitt 4.1.6; Tab. A, Tab. G).

Aufgrund der verbleibenden Unklarheiten hinsichtlich der taxonomischen Zuordnung wird die Sippe in dieser Arbeit als Crepis tectorum („outcrop-form“ sensu ANDERSSON 1990) angegeben.

\section{Festuca oelandica (Hackel) K. Richter}

Festuca oelandica aus dem F. rubra-Aggregat kommt nach MossBerg \& STENBERG (2003) bzW. JONSELL \& KARLSSON (2004: 159) endemisch auf Öland und Gotland vor. TUTIN et al. (1980) und LUNDQVIST (1986) erwähnen hingegen nur das Vorkommen auf Öland. Nach letzgenanntem Autor wächst die Art dort teilweise großflächig auf den zeitweise überschwemmten, flachgründigen und skelettreichen Böden der Alvare und wird häufig mit Festuca ovina verwechselt. Laut MOSSBERG \& STENBERG (2003) kommt sie auf diesen Standorten vorwiegend in den im Frühjahr nassen Senken vor und bildet dort kleine Horste. Die Pflanze erreiche eine Höhe von 10-20 cm, habe blau-grüne, 1,0-1,4 mm breite, steife und gebogene Blätter sowie 6-8 mm lange, kahle oder feinhaarige Ährchen. Nach TUTIN et al. (1980) wächst Festuca oelandica horstförmig mit kurzen Rhizomen, hat 20-37(-47) cm lange Halme sowie mehr oder weniger haarige, steife und spitze Blätter mit 5(-7) deutlich hervortretenden Blattrippen. Die Sclerenchymstränge seien gedrungen und zusammenfließend,die Blattscheide leicht rosa und geschlossen. Die Rispe erreicht laut Autoren eine Länge von 4$6 \mathrm{~cm}$, während die bläulich bis dunkel-violetten, bereiften Ährchen 6,5-8,7 mm lang werden. Die Hüllspelzen haben eine Größe von 2,8-4,9 mm $\times 1,1-1,3 \mathrm{~mm}$ und die spärlich behaarte Deckspelze sei 4-6,3 $\mathrm{mm} \times 1,6-2,3 \mathrm{~mm}$ groß.

Auf Saaremaa wuchsen auf der Alvarfläche bei Katri pank (X17) Pflanzen, welche der Diagnose der Autoren morphologisch entsprachen. Sie fielen unter anderem durch einen geschlossenen, mehrere Zelllagen dicken Sclerenchymring auf der Blattunterseite auf und kamen im Crepido-Allietum vor (Abschnitt 4.1.6; Tab. G).

Da die taxonomische Zuordnung der auf Saaremaa gesammelten Pflanzen bislang noch nicht abschließend geklärt ist, wird die Sippe in dieser Arbeit als Festuca cf. oelandica angegeben.

\section{Moose}

Folgende Moose habe ich während meiner Untersuchungen neu für Estland nachgewiesen:

\section{Bryum radiculosum Brid.}

Bryum radiculosum aus dem B. erythrocarpum-Komplex kommt nach NEBEL \& PHILIPPI (2001) in Europa vor allem in klimatisch günstigen Bereichen im mediterranen Raum, auf den Azoren, den Kanarischen Inseln, auf Madeira sowie in den westlichen und mittleren Teilen Europas vor. Als nördlichste bislang bekannte Fundorte werden Irland, Schottland und Dänemark, als östlichster der Kaukasus angegeben. Nach den Autoren wächst das Moos sehr 
dicht, beinahe kissenförmig und zeichnet sich durch seine rot-braunen mehrzelligen $120 \mu \mathrm{m}-$ $180 \mu \mathrm{m}$ großen Brutkörper und die ungesäumten Blätter aus. Bryum radiculosum komme an sommerwarmen Stellen auf kalk-, basen- und humusreichen Böden vor.

Auf Saaremaa habe ich die Art auf Alvarflächen westlich von Kuressaare (N04, N06) sowie bei Iipla (Z21) auf Standorten im Crepido-Allietum (Abschnitt 4.1.6; Tab. G), die den Angaben der Autoren entsprechen, gesammelt. Saaremaa scheint, bezogen auf die Angabe von NeBEl \& PHILIPPI (2001), die nordöstliche Verbreitungsgrenze der Art zu sein.

\section{Tortula calcicolens W. A. Kramer}

Tortula calcicolens aus dem T. ruralis-Aggregat kommt nach NeBEL \& PHILIPPI (2000) auf Madeira, in West-, Zentral- und Südeuropa sowie in Nord- bis Südskandinavien vor. Sie wachse an sonnigen, trockenen bis mäßig frischen, kalkreichen oder auch nur basenreichen Stellen vor allem auf flachgründiger, steiniger Erde oder dünnen Humusauflagen über Gestein. Außerdem komme sie häufig auf synanthropen Substraten vor. Die Art unterscheidet sich nach den Autoren von T. ruraliformis (Besch.) Grout und T. ruralis (Hedw.) P. Gärtn. durch die mamilös aufgewölbten Laminazellen und die in der Mitte der Zellen zentriert liegenden hufeisenförmigen Papillen, wodurch die Zellgrenzen deutlich erkennbar sind.

Auf Saaremaa habe ich einen großen Bestand von Tortula calcicolens westlich von Katri pank (X77) auf einem südwestlich exponierten Lesesteinwall über stark humosem, mäßig carbonathaltigem, jedoch basenreichen Substrat im Cladonio-Sedetum (Abschnitt 4.1.5; Tab. F) gefunden. Dies stimmt völlig mit den Standortangaben von NeBEL \& PHILIPPI (2000) überein. Bezüglich der Verbreitungsangaben der Autoren dürfte die Art auf Saaremaa an ihre nordöstliche Verbreitungsgrenze stoßen.

\section{Flechten}

Folgende Flechte habe ich während meiner Untersuchungen neu für Estland nachgewiesen:

Agonimia globulifera Brand \& Diederich.

Nach SÉRUSIAUX et al. (1999) wächst diese unscheinbare Flechte über Moosen sowie anderen Flechten, auf Sand oder seltenen Kalkfelsen. Sie falle erst bei starker Vergrößerung durch zahlreiche glänzend schwarze sterile Kügelchen $(70-200 \mu \mathrm{m})$ auf. Die Perithecien seien relativ selten und enthielten große $(37-50 \mu \mathrm{m} \times 16-26 \mu \mathrm{m})$, mauerförmige Ascosporen mit 35-60 Zellen (SÉRUSIAUX et al. 1999).

Auf Saaremaa wächst die Art über Moosen, direkt auf dem humusreichen Erdboden oder sie geht auf tote organische Materialien und kalkhaltige Gesteine über (SUIJA et al. 2005). Ich habe sie dort in sämtlichen Alvargebieten nachgewiesen. Sie ist mit einer Stetigkeit von $31 \%$ territoriale Charakterart des Crepido-Allietum (Abschnitt 4.1.6; Tab. A, Tab. G). Auf Öland wurde Agonimia globulifera ebenfalls in einem Alvargebiet (bei dem See Möckelmossen, Stora alvaret) über kalkhaltigem Gestein gefunden (SANTESSON et al. 2004).

\section{Zusammenfassung der Besonderheiten der Flora}

Ich konnte insgesamt vier neue Arten für Estland nachweisen. Drei davon sicher, bei einer ist die taxonomische Zuordnung der gesammelten Pflanzen noch nicht abschließend geklärt. Die Neufunde sind durch eine Gefäßpflanzenart, zwei Moosarten und eine Flechtenart vertreten. Gründe für die bisherige Nichtbeachtung könnte die Verwechslung mit morphologisch ähnlichen Taxa oder die Unscheinbarkeit der Arten sein. Bei zwei weiteren Arten konnte ich früheren Vermutungen estnischer Autoren entgegen anderslautenden Verbreitungsangaben in 
der schwedischen Literatur zustimmen. Die Funde dieser Sippen unterstreichen weiterhin die floristische Beziehung zu Öland.

\subsection{Ursachen der Phytodiversitätsmuster}

Die Muster der Phytodiversität sind generell sehr komplex. Es existieren zahlreiche Studien, welche die Zusammenhänge zwischen kleinräumiger Artenvielfalt und verschiedensten standörtlichen, biologischen und historischen Parametern untersuchten und dabei nicht immer zu einheitlichen Ergebnissen kamen (GRACE 1999). Im Folgenden werden die eigenen Untersuchungen im Kontext einer Auswahl der bereits vorliegenden Hypothesen diskutiert. Dabei liegt ein Fokus auf der Fragestellung, wie sich der im europaweiten Vergleich überdurchschnittlich hohe Artenreichtum in den Trockenrasen der Insel Saaremaa erklären lässt (detaillierter Vergleich der Artenvielfalt europäischer Trockenrasen in DENGLER 2005).

\subsubsection{Abiotische Umweltparameter}

Die abiotischen Umweltparameter (Boden, Klima, geografische Lage) bestimmen die Lebensbedingungen eines Standortes und beeinflussen somit entscheidend dessen Artenkombination und -diversität (z. B. BEGON et al. 1998: 601 ff.). Dies verdeutlichen die unterschiedlich hohen Artendichten (vgl. 4.2.3) der standörtlich meist deutlich voneinander getrennten Trockenrasentypen (vgl. 4.2.1). Die abiotischen Umweltparameter stellen die basale Voraussetzung einer Lebensgemeinschaft dar und werden deshalb zuerst bearbeitet.

Im Folgenden soll unter Einbeziehung der in Abschnitt 4.3.1 erhaltenen Ergebnisse sowie einer Auswahl relevanter Literatur die Frage geklärt werden, welchen Einfluss abiotische Parameter auf den Gesamtartenreichtum haben, und ob der Artenreichtum der Artengruppen in unterschiedlicher Weise beeinflusst wird.

\section{Bodentiefgründigkeit}

In den multiplen Regressionsmodellen für die Gesamt- und Gefäßpflanzenartendichte zeichnet sich eine Optimumskurve, in jenem für die Moosartendichte ein linear negativer Zusammen-hang in Bezug zu der Bodentiefgründigkeit ab (vgl. 4.2.1).

In das Modell für die Flechtenartendichte wurde dieser Parameter nicht aufgenommen, obwohl bei der einfachen Regression ein signifikant positiver Zusammenhang festzustellen war (Tab. 38). Dies könnte auf die starke Wechselbeziehung zwischen der Bodentiefgründigkeit und der Deckung der Gesteine sowie dem Gesteinsanteil zurückzuführen sein, wobei Letztgenannter in das multiple Modell aufgenommen wurde (Tab. 37). Außerdem besiedeln viele Flechtenarten Extremstandorte (SCHÖLLER 1997).

LÖBEL et al. (eingereicht) konnten auf der Insel Öland ebenfalls eine Beziehung zwischen der Tiefgründigkeit und den Gesamt- bzw. Kryptogamenartendichten nachweisen. Dort wurde jedoch eine Minimumverteilung der Werte mit einem Maximum bei sehr flachen und einem leichten Anstieg der Artendichten bei extrem tiefgründigen Böden festgestellt (LÖBEL 2002: 150). Auf Saaremaa sinken die Gesamt- und Gefäßpflanzenartendichten bei sehr flachgründigen Böden, wie sie im Cladonio-Sedetum vorkommen, etwas ab. Das Maximum hingegen liege im Crepido-Allietum auf etwas tiefgründigeren Böden. Auf Öland sei dieser Abfall auf extrem flachgründigen Böden ebenfalls hinsichtlich der Kryptogamenartendichten festzustellen (LÖBEL et al. eingereicht).

Die Abhängigkeit der unterschiedlichen Artendichten von der Bodentiefgründigkeit, die durch einen deutlichen Rückgang der Gefäßpflanzenartendichten bei extrem tief- wie auch flachgründigen Böden charakterisiert ist, könnte unterschiedliche Ursachen haben und mit den Bodeneigenschaften der vorherrschenden Bodenarten zusammenhängen. Bei sehr tief- 
gründigen Böden könnte der Effekt auf das ungünstige Wasser- und Nährstoffhaltevermögen der Böden zurückzuführen sein, bei extrem flachgründigen Böden hingegen auf den begrenzten Wurzelraum sowie die extremen Lebensbedingungen insgesamt.

Die tiefgründigsten Böden der Trockenrasen auf Saaremaa bestehen aus reinem Sand. Nach BLUM (1992: 34) sind Böden mit einer einseitigen Korngrößenzusammensetzung generell ungünstig. Bezogen auf Sandböden sei das Wasser- und Nährstoffhaltevermögen sehr gering. Insbesondere Gefäßpflanzen sind jedoch als homoiohydre Organismen auf eine regelmäßige und ausreichende Wasserzufuhr angewiesen, und die wenigsten Arten sind durch Anpassungsstrategien in der Lage, bei Trockenheit zu überdauern (z. B. SCHULzE et al. 2002: 133 ff., 325 ff.). Die relativ niedrigen mittleren Gefäßpflanzenartendichten der Koelerio-Corynephorenea-Gesellschaften, insbesondere jene der Caricetum arenariae, Helichryso-Jasionetum und Festucetum polesicae, wären so erklärbar.

Bei den flachgründigen Böden des Cladonio-Sedetum könnte die Ursache der unterdurchschnittlichen Artendichten in den extremen Lebensbedingungen liegen. Laut TYLER (1996) ist der Wurzelraum bei sehr flachgründigen Böden begrenzt. Es ist also anzunehmen, dass ebenso das pflanzenverfügbare Wasser limitiert ist, zumal an heißen Tagen die dünne Bodenschicht sehr schnell austrocknet. Nach TYLER (1996) sind insgeamt weniger und vorwiegend Anuelle sowie Arten mit reduzierter Blattmasse an diese Bedingungen angepasst. In der Artenzusammensetzung der Bestände des Cladonio-Sedetum auf Saaremaa bestätigt sich diese Diagnose (z. B. Sukkulente oder die gegen das Crepido-Allietum differenzierenden Therophyten; Tab. A).

Für Gefäßpflanzen scheinen somit die Wasser- und Nährstoffverhältnisse in den schluffigen oder lehmigen, mitteltiefgründigen Böden der Helictotrichon-Gesellschaft am günstigsten zu sein. Vielfach wurde bei der Beziehung zwischen lokaler Pflanzenartendichte und Produktivität eine Optimumkurve mit einem Maximum bei intermediären Verhältnissen festgestellt (RoSENZWEIG \& ABRAMSKY 1993, WAIDE et al. 1999). Eine sehr hohe Produktivität (z. B. durch Eutrophierung) bedingt einen Vorteil konkurrenzstarker Arten, wodurch es zu einem Ausschluss konkurrenzschwächerer kommen kann. Dies hat einen Rückgang der Arten-dichten zur Folge (z. B. Новонм 2000, 2005). Die hohen Gefäßpflanzenartendichten in den Halbtrockenrasen Saaremaas lassen somit auf intermediäre Nährstoffverhältnisse schließen, bei denen eine hohe Anzahl von Arten nebeneinander ko-existieren können. Auf sehr flach- sowie tiefgründigen Böden kann sich die Trockenheit negativ auf die Verfügbarkeit von Nähr-stoffen auswirken, wodurch die geringere Produktivität mit geringeren Gefäßpflanzenarten-dichten in diesen Lebensräumen zu erklären wäre.

Die meisten Moosarten können laut FRAHM (2001: 238 f.) als poikilohydre Organismen Trockenperioden überstehen. Der linear negative Zusammenhang zwischen Bodentiefgründig-keit und Moosartendichte muss somit andere Gründe haben. Eine Erklärungsmöglichkeit wäre, dass der geringe Humusgehalt der Böden (s. u.) in den Sandtrockenrasen, welcher mit der Tiefgründigkeit in negativer Beziehung steht (Tab. 19), die Lebensbedingungen für viele Moosarten verschlechtert. Außerdem könnte sich die Instabilität des Sandbodens (s. u.) sowie die Übersandung negativ auf viele Arten auswirken.

\section{Überstauung}

Im Gegensatz zu den nicht überstauten Trockenrasen ist ein linear positiver Einfluss der winterlichen und nach starken Regenfällen auftretenden Überstauung der Alvarflächen auf die Gesamt- sowie Kryptogamenartendichten zu verzeichnen. Sie stellt in den multiplen Regressionsmodellen den einflussreichsten Parameter dar. Auch andere Autoren (z. B. PÄRTEL et al. 1999a) berichten von der Überstauung der Alvarflächen Saaremaas. Dennoch ist dieser Parameter mit Vorsicht zu interpretieren, da ich dieses Ereignis teilweise nur an den unter Abschnitt 3.1.2 erläuterten Indizien bestimmen konnte. Es ist anzunehmen, dass die 
Überstauung ähnliche Effekte hervorruft wie beispielsweise in den Alvargebieten der Insel Öland. Sie führt dort zu Frosthebungserscheinungen, die ein ausgeprägtes Mikrorelief bedingen (ALBERTSON 1950). Dieses Phänomen ist auch in den Beständen des CrepidoAllietum auf Saaremaa festzustellen. Die positive Korrelation mit den Artendichten könnte sich durch den Störungseffekt der Frosthebungen sowie die Erosionswirkung des Wassers erklären lassen. Es werden stetig neue Pionierstandorte wie blanke Erdböden oder frei liegende Steine geschaffen, die rasch von einer Reihe epigäischer und epilithischer Kryptogamen besiedelt werden. Aufgrund des hohen Anteils der Kryptogamen am Gesamtartenreichtum (vgl. Abschnitt 4.2.3) ist festzustellen, dass sich die Überstauung positiv auf die Gesamtartendichten auswirkt.

\section{Stabilität des Untergrundes}

In den häufig überschwemmten Alvargebieten Ölands scheinen sich die Frosthebungen deutlich stärker auf die Stabilität des Bodens auszuwirken als auf Saaremaa. Nach LöBEL et al. (eingereicht) werden dort insbesondere langsam wachsende Flechten beeinträchtigt, was sich in geringeren mittleren Flechtenartendichten niederschlägt. Das von Frostwechseln und Überstauung stark negativ beeinflusste Crepido-Allietum zeigt auf Öland außerdem die geringsten Gesamtartendichten der Tortello-Helianthemenion-Gesellschaften (DENGLER \& LÖBEL 2006). Durch die Frosthebungen kann es zur Schädigung der Pflanzenwurzeln kommen, was wiederum zum Ausfall einiger Arten führt. Auf Saaremaa konnte dieser negative Effekt auf Flechten und Gefäßpflanzen jedoch nicht in dem Ausmaß festgestellt werden.

CONNELL (1978) beschreibt die ,intermediate disturbance hypothesis“ wonach die Artenvielfalt durch moderate Störungen aufgrund einer geminderten interspezifischen Konkurrenz maximiert wird. Dies wäre ein möglicher Erklärungsansatz: Frosthebungen stellen eine moderate Störung dar. An einigen Stellen des Lebensraumes werden Pflanzen beeinträchtigt oder sterben ab. Dies könnte zu einer Verminderung der Konkurrenzverhältnisse des Lebensraumes führen, sich förderlich auf die Koexistenz von Arten sowie positiv auf die Artendichten auswirken. Diese Dynamik deckt sich mit dem ,carousel-model“ von VAN DER MAAREL \& SYKES (1993). Danach sterben ständig Pflanzen bzw. deren oberirdische Teile ab, was regelmäßig Lücken in der Vegetation entstehen lässt. Diese werden alsdann aus dem Artenpool besetzt. Nach HUBER (1994: 478) geht die Wiederbesiedlung solcher Lücken relativ schnell vor sich, da viele „Alvarpflanzen“ eine sehr gute Regeneration aus Samen, Rhizomen oder Zwiebeln zeigen.

In den Trockenrasenbeständen der Koelerio-Corynephorenea auf den Dünen Saaremaas scheint der Boden teilweise noch weitaus instabiler zu sein als auf den Alvarflächen. Dort ist eine stärkere Dynamik durch Sandverlagerung und -verwehung zu erkennen, wodurch es außer der oben angesprochenen Trockenheit noch zu einer mechanischen Belastung der Pflanzen durch Übersandung oder verwehte Sandkörner kommen kann. Die Vorraussetzung, solche Standorte besiedeln zu können, wäre beispielsweise die Fähigkeit, die obersten Zentimeter des Sandes festlegen zu können bzw. die Sandverwehungen schadlos überstehen zu können. DOLNIK (2003: 102) konnte bei einigen dicht und flächig oder krustig wachsenden Moosen und Flechten in den Sandtrockenrasen der Kurischen Nehrung die Festlegung der obersten Sandschicht bestätigen. In den Sandtrockenrasen Saaremaas habe ich dies ebenfalls bei einigen zumeist dicht wachsenden oder kräftigen Arten wie Ceratodon purpureus, Cephaloziella divaricata oder Tortula ruraliformis festgestellt. Die meisten Arten, so vermutlich auch die zahlreichen winzigen akrokarpen Moose, welche in den Felsgrusfluren wachsen, sind daran wahrscheinlich nicht angepasst. 


\section{Mikrorelief}

Der deutliche Unterschied in der Ausprägung des Mikroreliefs in den Beständen des CrepidoAllietum ist das Ergebnis der oben erwähnten Frostwechseleffekte sowie der Wassererosion bei Überstauungsereignissen. Untersuchungen in Trockenrasengesellschaften auf Öland konnten einen positiven Einfluss eines ausgeprägten Mikroreliefs auf den Gefäßpflanzen- und Moosartenreichtum verdeutlichen, während der Flechtenartenreichtum bei mittleren Werten am höchsten war (LÖBEL et al. eingereicht). Auf Saaremaa ist ebenfalls ein positiver Effekt bei stärker reliefiertem Boden bezogen auf die Gesamt-, Moos- und Flechtenartendichte festzustellen.

Nach BEGON et al. (1998: 607 f.) hat eine hohe kleinstandörtliche Heterogenität einen positiven Einfluss auf den Artenreichtum. Ein ausgeprägtes Mikrorelief könnte ebendiesen Effekt durch das Vorhandensein verschiedener Mikrohabitate bedingen. Nach einem simulierten Modell von MOORE et al. (2001) wäre dem Pflanzenartenreichtum durch die steigende Heterogenität eines Lebensraumes theoretisch keine Grenzen gesetzt. LUNDHOLM \& LARSON (2003) konnten jedoch auch in einer empirischen Studie den positiven Effekt der kleinstandörtlichen Heterogenität auf den Gefäßpflanzenartenreichtum in einem Alvargebiet in Süd-Ontario (Canada) nachweisen, indem sie unter anderem die Anzahl der Mikrohabitate einer Aufnahmefläche dokumentierten und diese in Relation zur Artenvielfalt setzten. Auch in den Alvaren Saaremaas ist eine solche Untergliederung in Mikrohabitate zu erkennen. Auf den ständig trockenen Bulten ist beispielsweise eine andere Artenkombination als in den wechselfeuchten Senken oder dem Übergang zwischen diesen beiden Mikrohabitaten vorhanden.

Eine Nischendifferenzierung auf räumlicher (Besiedelung von Mikrohabitaten), funktionaler (unterschiedliche Ressourcennutzung), sowie zeitlicher (Jahreszeiten, unterschiedliche Reproduktionsintervalle) Ebene kann nach verschiedenen Autoren (z. B. ScHUlzE et al. 2002: 736 ff., BAROT \& GignOUX 2004, FREY \& LÖSCH 2004: 258 ff.) die Koexistenz zahlreicher Arten ermöglichen. Dadurch kann die Artenvielfalt eines Lebensraumes erhöht werden (GRUBB 1977, SCHULZE et al. 2002: 671). Das Nebeneinander verschiedener Mikrohabitate könnte den positiven Effekt auf die Kryptogamenartenvielfalt erklären. Eine Reihe von Moosen und Flechten zeigen eine stärkere Nischendifferenzierung (FRAHM 2001: 157, 229), Spezialisierung sowie Bindung an mikroklimatische und standörtliche Verhältnisse (SCHÖLLER 1997). Insbesondere die erwähnten Feuchtegradienten können nach ZAMFIR et al. (1999) die Verteilung verschiedener Kryptogamenarten innerhalb eines Lebensraumes beeinflussen.

\section{Humusgehalt des Bodens}

Der unimodale Zusammenhang zwischen dem Humusgehalt des Bodens und den Gesamt-, Gefäßpflanzen- sowie Moosartendichten bei den einfachen Regressionsanalysen könnte zum Teil auf die negative Beziehung zu der Tiefgründigkeit zurückzuführen sein.

WILD (1995: 182) schreibt dem Humusanteil eines Bodens einen positiven Einfluss auf die physikalischen, chemischen und biologischen Eigenschaften von Böden zu (Wasserhaltevermögen, Verbesserung der Bodenstruktur, besserer Zusammenhalt der Bodenpartikel, günstigere Keimungsbedingungen, Pufferwirkung u. a.).

Auf Saaremaa liegt der Humusgehalt an Standorten mit sehr flachgründigen Böden in einem günstigen Bereich, die Menge der Bodenauflage stellt hier vermutlich den limitierenden Faktor dar (s. o., Tiefgründigkeit). Die Bestände des Crepido-Allietum wachsen auf den humusreichsten Böden, wodurch das Wasserhaltevermögen des Bodens und somit das Mikroklima günstiger sein könnte. Es ist folglich möglich, dass verschiedene Moosarten 
davon profitieren, wodurch zum einen die hohen Moosartendichten, zum anderen der hohe Anteil der Moose am Gesamtartenreichtum zu erklären wäre.

Die gefäßpflanzenartenreichen Halbtrockenrasen wachsen auf weniger extremen Standorten mit intermediären Lebensbedingungen. Bezogen auf die Aussage von WILD (1995: 182), könnte der relativ günstige Humusanteil einen positiven Einfluss darauf haben.

Nach WILD (1995: 182) verbessert Humus den Zusammenhalt der Bodenpartikel. Der geringe Humusgehalt der tiefgründigen Sandböden ist deshalb mitverantwortlich für die Mobilität des Sandes. Er stellt somit einen weiteren Faktor zur Erklärung dieses komplexen Beziehungsgefüges hinsichtlich der geringeren Artendichten in den auf Dünen wachsenden Sandtrockenrasenbeständen dar.

\section{Gesteinsanteil des Bodens}

Der Anteil der im Boden enthaltenen Gesteine bestimmt entscheidend das Volumen des für die Pflanzen durchwurzelbaren Bodens. Der linear negative Zusammenhang zwischen der Gefäßpflanzenartenvielfalt und dem Anteil des Bodenskeletts im multiplen Regressionsmodell könnte wiederum durch die ungünstigeren Wasser- und Nährstoffverhältnisse bei höheren Gesteinsanteilen erklärt werden.

Der linear positive Zusammenhang zwischen Gesteinsanteil und Flechtenartenvielfalt lässt sich auf die Theorie zurückführen, dass Flechten Extremstandorte besiedeln können, die für Gefäßpflanzen ungünstig sind (zu trocken, zu nährstoffarm, usw.) (ScHÖLLER 1997). Als poikilohydre Organismen sind viele Flechtenarten weitgehend unabhängig von den Wasserverhältnissen des Bodens und somit persistent gegen Trockenperioden (ZAMFIR et al. 1999).

\section{Deckungsgrad freiliegender Gesteine}

Gesteine stellen ein geeignetes Substrat für viele epilithische Flechtenarten dar (SCHÖLLER 1997). Der Einfluss von Sonderstandorten auf die Artenvielfalt wurde bereits verschiedentlich untersucht und die Bedeutung dieser für die Artenvielfalt nachgewiesen (z. B. DoLNIK 2003: 108 f.). Die freiliegenden Gesteine erhöhen somit die kleinstandörtliche Heterogenität, wodurch es zu einer „Habitaterweiterung“ kommt. Der linear positive Zusammenhang bezüglich der Flechtenartenvielfalt könnte darauf zurückzuführen sein.

Allerdings sollte dieser Parameter nicht losgelöst von den anderen aufgenommenen Variablen betrachtet werden, da eine starke Korrelation mit der Tiefgründigkeit und dem Gesteinsanteil festzustellen ist, wodurch sich die beschriebenen Effekte überlagern könnten.

\section{pH-Wert des Bodens}

Offenlandgesellschaften auf sauren Böden sind nach GRIME (1973, 2001: 294 ff.) in Europa in der Regel durch niedrigere Artenzahlen charakterisiert als solche auf basischen Böden. Dies wird von EWALD (2003) durch die Theorie erklärt, dass in Südwestasien sowie Südosteuropa ausgedehnte Steppengebiete auf basischen oder neutralen Böden existierten. Im Zuge des Artbildungsprozesses während der Evolution haben sich zahlreiche Pflanzenarten an diese Bedingungen angepasst. Diese Arten konnten sich nach PÄRTEL (2002) ausbreiten und über die während des Pleistozäns „erneuerten“ Gebiete mit kalkreichen Böden nach Europa einwandern. Aus diesem Grund haben nach EWALD (2003) Basiphyten heutzutage in Europa einen weitaus größeren Anteil am Gesamtarteninventar als Azidophyten. Von verschiedenen Autoren wurde dabei ein positiver Zusammenhang zwischen dem $\mathrm{pH}-$ Wert und der lokalen Artendichte in Trockenrasen festgestellt (TYLER 1996, DENGLER 2004c, PÄRTEL 2002), während andere diese Beziehung nicht nachweisen, jedoch den positiven Einfluss auf die 
Größe des Artenpools bestätigen konnten (z. B. CHYTRÝ et al. 2003). Bei Untersuchungen von LÖBEL et al. (eingereicht) auf Öland stellte sich der $\mathrm{pH}$-Wert sogar als einflussreichster Parameter auf die Artenvielfalt heraus. DENGLER (2005) geht von einem unimodalen Zusammenhang aus, wobei die Artendichte bis zu einem gewissen $\mathrm{pH}-$ Wert ansteigt und danach wieder abfällt. Nach WILD (1995: 208) nimmt bei niedrigen $\mathrm{pH}$-Werten $(<5,5)$ durch den Überschuss an $\mathrm{H}^{+}$-Ionen zum einen die Fähigkeit der Böden zur Adsorption von Kationen $\mathrm{ab}$, wodurch Nährstoffe wie $\mathrm{Ca}^{2+}$ und $\mathrm{Mg}^{2+}$ in Lösung gehen und ausgewaschen werden. Nach TYLER (1996) gehen zum anderen bei einem $\mathrm{pH}$-Wert $(\mathrm{KCl})$ von weniger als 4,5 phytotoxische $\mathrm{Al}^{3+}$-Ionen in Lösung. Ebenso bestehen laut ihm Wechselwirkungen zwischen diesen $\mathrm{Al}^{3+}$-Ionen und der Kationenaustauschkapazität von Böden. Nach WILD (1995: 208) werden dadurch wichtige Pflanzennährstoffe von ihren Austauschplätzen verdrängt und stehen den Pflanzen somit nicht mehr zur Verfügung. Zudem lässt nach BLUM (1992: 80) in höheren Bereichen des $\mathrm{pH}$-Spektrums die Pflanzenverfügbarkeit wichtiger Makronährstoffe nach. Laut TYLER (1996) wirkt sich dies vor allem auf die Phosphatverfügbarkeit der Böden aus. Bei pH-Werten $(\mathrm{KCl})$ über 5,5-6,0, also besonders bei kalkreichen Böden, liegen nach ihm sämtliche anorganischen Phosphat-Ionen in Form von unlöslichen Ca-Phosphaten vor, weshalb diese von den Pflanzen vor der Aufnahme umgewandelt werden müssen. Es ist folglich anzunehmen, dass die Trockenrasen der Insel Saaremaa aufgrund der zumeist kalkhaltigen Böden mit insgesamt relativ hohen $\mathrm{pH}$-Werten P-limitiert sind. Ferner ist möglich, dass deshalb die Produktivität der Trockenrasen auf einem Niveau verbleibt, bei dem konkurrenzstarke Arten ihren Vorteil noch nicht ausspielen können.

Bei den eigenen Untersuchungen wurde der $\mathrm{pH}$-Wert nicht in die multiplen Regressionsmodelle aufgenommen. In den einfachen Regressionsanalysen zeichnete sich jedoch der oben beschriebene unimodale Zusammenhang bezüglich der Gesamt,- Gefäßpflanzen- und Moosartendichten ab, was sich durch die angeführten Theorien erklären lässt.

Allerdings fehlen im Aufnahmematerial Trockenrasen auf extrem sauren Böden, da diese auf Saaremaa nicht vorkommen. Die Ursache liegt darin, dass potenzielle Standorte im Untersuchungsgebiet noch relativ jung (vgl. Abschnitt 2.2) und bislang wenig entkalkt sind. Die angesprochene $\mathrm{Al}^{3+}$-Toxizität dürfte somit in den Trockenrasen Saaremaas keine Rolle spielen. Extrem alkalische Böden fehlen ebenso. In sämtlichen Aufnahmen habe ich $\mathrm{pH}-$ Werte in einer Spanne von 4,6-8,2 gemessen, was einer relativ kurzen Gradientenlänge von 3,6 entspricht. DUPRÉ et al. (2002) konnten jedoch durch den Vergleich von 15 Studien nachweisen, dass bereits ab einer Gradientenlänge von drei die besagten unimodalen Zusammenhänge nachgewiesen werden, während darunter liegende Werte zu einer linearen Beziehung führen. Die eigenen Untersuchungen entsprechen somit diesem Ergebnis.

\section{Carbonatgehalt des Bodens}

Der Carbonatgehalt beeinflusst den pH-Wert der Böden und ist deshalb mitverantwortlich für den im vorausgegangenen Abschnitt erläuterten positiven Zusammenhang zwischen dem $\mathrm{pH}-$ Gradienten und dem Artenreichtum in mitteleuropäischen Trockenrasen. In den eigenen Untersuchungen ist dieser Zusammenhang jedoch offensichtlich nicht für alle Artengruppen zutreffend. Zwischen dem Carbonatgehalt und den Flechtenartendichten ist in den Trockenrasen Saaremaas eine linear negative Beziehung festzustellen. Von Gefäßpflanzen ist bekannt, dass ein Überschuss an $\mathrm{Ca}^{2+}$-Ionen schädlich wirken kann (REISIGL \& KELLER 1994: 12). Es wäre zu vermuten, dass dieser negative Effekt auch bei vielen Flechtenarten zum Tragen kommt. Flechten können Mineralien durch sämtliche Teile ihres Thallus aufnehmen und diese effizient akkumulieren. Es werden jedoch auch schädliche Substanzen absorbiert, die den Photobionten schädigen (BRODO et al. 2001: 39).

Es ist somit möglich, dass Azidophyten und Basiphyten bis zu einer bestimmten Carbonatkonzentration nebeneinander vorkommen können. Höher liegende Werte könnten zunächst 
zum Ausfall der Azidophyten führen, extrem hohe Werte das alleinige Vorkommen spezialisierter Arten bedingen.

\section{Kationenaustauschkapazität und Basensättigung}

Die Kationenaustauschkapazität ist nach WILD (1995:197 ff.) ein Maß für die Fähigkeit des Bodens Kationen zu binden. Sie dient in Verbindung mit dem pH-Wert dazu, Aussagen über die Pufferfähigkeit eines Bodens zu treffen. Übertragen auf die eigenen Untersuchungen wäre die Pufferfähigkeit in den Böden der Sedo-Scleranthenea am höchsten und in den KoelerioCorynephorenea am niedrigsten. Die Basensättigung dient laut WILD (1995: 201) neben der Messung des pH-Wertes zur Abschätzung einer negativen Auswirkung der Bodenazidität auf das Pflanzenwachstum. Die Tatsache, dass die Basensättigung in sämtlichen Trockenrasengesellschaften Saaremaas bei nahezu $100 \%$ liegt, schließt diesen negativen Einfluss jedoch aus.

\section{Größe der Trockenrasengebiete}

Bei Untersuchungen von LÖBEL et al. (eingereicht) auf Öland ergab sich ein positiver Zusammenhang zwischen der Größe der Trockenrasengebiete sowie der Gesamt-, Moos- und Flechtenartenvielfalt auf $4 \mathrm{~m}^{2}$ großen Flächen. Die Gefäßpflanzenartenvielfalt wurde jedoch nicht signifikant beeinflusst. KRAUSS et al. (2004) hingegen stellten keinen Zusammenhang zwischen der Größe des Gebietes und der Artenvielfalt auf einheitlichen Flächengrößen in Kalkmagerrasen im Raum Göttingen fest. HELM et al. (im Druck) vermuten aufgrund der fortschreitenden Fragmentierung der Alvargebiete Saaremaas und dem daraus resultierenden Habitatverlust einen Rückgang der Biodiversität.

Die eigenen Untersuchungen ergaben einen positiven Zusammenhang zwischen Gebietsgröße und Flechtenartenvielfalt, was den Ergebnissen von LöBEL et al. (eingereicht) und HELM et al. (im Druck) entspricht. Das multiple Regressionsmodell stellt hingegen eine Minimumverteilung der Werte für die Gefäßpflanzenartendichte dar. Dieses Ergebnis könnte über Randeffekte erklären zu sein. HELM et al. (im Druck) konnten auf Saaremaa nachweisen, dass Arten aus angrenzenden Lebensräumen in kleine, stärker fragmentierte und isolierte Alvare einwandern, was die höheren Artenzahlen auf kleineren Flächen erklären könnte.

\section{Geografische Breite}

Nach Begon et al. (1998: 611) nimmt der Artenreichtum vom Äquator zu den Polen ab. Dies gilt nach DENGLER (2005) jedoch nicht für die Artendichten in Trockenrasen. Die Artendichten nordeuropäischer Trockenrasengesellschaften liegen laut ihm tendenziell etwas höher als in vergleichbaren mitteleuropäischen Vegetationstypen. Im Vergleich zu den in DENGLER (2005) angeführten Artendichten auf $4 \mathrm{~m}^{2}$ liegen die eigenen festgestellten Werte über jenen vergleichbarer mitteleuropäischer Trockenrasen. Dies entspricht somit der Aussage von DENGLER.

\section{Klima}

Das Klima ändert sich mit der geografischen Breite. Je höher der Breitengrad, desto stärker machen sich saisonale Klimaschwankungen bemerkbar. Diese setzen nach BEGON et al. (1998: 609) eine Nischendifferenzierung auf zeitlicher Ebene voraus. Die Dauer der Vegetationsperiode bestimmt laut Autoren (1. c.: 603) ferner die Produktivität eines Lebensraumes.

Dies könnte man als einen Grund für die höheren Artendichten der nordeuropäischen Trockenrasen anführen, da viele Arten aufgrund der kürzeren Vegetationsperiode eine deutlich geringere Wuchshöhe als in Mitteleuropa erreichen (eigene Beobachtung: z. B. 
Pseudolysimachion spicatum). Ebenso wirken sich nach ZoBEL (1992) sämtliche Parameter, welche die vertikale Heterogenität einer Pflanzengesellschaft vermindern, durch die Reduktion der Lichtkonkurrenz zwischen den Arten positiv auf die Artenvielfalt aus.

Außerdem könnten die lokalen Klimaunterschiede auf Saaremaa geeignete Parameter darstellen, um einen Teil der unerklärten Restvarianz in den Artendichten verständlich zu machen. Die artenreichsten Alvarflächen befinden sich im westlichen Teil Saaremaas. Dort sind die durchschnittlichen Temperaturen etwas milder (Tab. 1) und es fällt unwesentlich weniger Niederschlag als im Osten. Während der Vegetationsperiode sind keine signifikanten Unterschiede zu verzeichnen (Tab. 2). Die möglicherweise ausschlaggebenden Unterschiede könnten indes in der etwas kürzeren Schneebedeckungsdauer im Winter und den höheren durchschnittlichen Windgeschwindigkeiten im Westen der Insel bestehen. Es wäre außerdem zu vermuten, dass der Schnee auf den Alvarflächen mit einer lückigen Vegetation aufgrund des offen liegenden, dunklen Bodens durch die stärkere Absorption der Sonnenstrahlung schneller abtaut und dadurch die schneefreie Zeit im Winter länger ist, als die angegebenen Durchschnittswerte wiedergeben. Nach LöBEL (2002: 11) ist der Groß-Alvar auf Öland ganzjährig schneefrei. Durch die kürzere Schneebedeckungsdauer hätten zumindest Kryptogamen die Möglichkeit Photosynthese $\mathrm{zu}$ betreiben und sich $\mathrm{zu}$ reproduzieren. Des Weiteren ist anzunehmen, dass die Gefäßpflanzen dort bei noch gefrorenem Boden und Lufttemperaturen über dem Gefrierpunkt einer erhöhten Stresswahrscheinlichkeit durch Frosttrocknis ausgesetzt sind. Die höheren Windgeschwindigkeiten könnten diesen Effekt im Winter durch eine erhöhte Transpirationsrate verstärken und sich zudem in den Trockenperioden im Sommer durch eine erhöhte Evapotranspirationsrate negativ auf den Wasserhaushalt der Gefäßpflanzen auswirken. Außerdem könnten die häufig auftretenden Spätfröste einen negativen Einfluss auf Gefäßpflanzen haben (MATUSZKIEWICZ zit. n. НовOHM 1998: 40). Aufgrund dieser Störungen wäre es möglich, dass einerseits die interspezifische Konkurrenz und andererseits die Sukzessionsgeschwindigkeit durch die Beeinträchtigung von immergrünen Gehölzen während eines strengen Winters gemindert werden. Die hohen Windgeschwindigkeiten könnten zusätzlich an exponierten Stellen durch eine verstärkte Erosionswirkung dazu beitragen, die Sukzession zu verlangsamen bzw. zu minimieren. Diese Vermutungen könnten Hinweise auf den besseren Erhaltungszustand der Alvare im Westen der Insel geben und die hohen Artendichten in den Alvargebieten bei etwas geringeren Gefäßpflanzenartendichten in diesen Gesellschaften erklären. Um genaue Aussagen darüber treffen zu können, müssten jedoch weiterführende Untersuchungen unter Einbeziehung weiterer Parameter wie dem Einfluss der kulturhistorischen Nutzungsformen angestellt werden.

\subsubsection{Biotische Interaktionen}

Die biotischen Umweltparameter stellen die biologischen Kennzeichen einer Pflanzengesellschaft dar. Die von den Organismen verursachte räumliche Heterogenität in Form einer Vegetationsstruktur sowie die gegenseitigen Interaktionen wie Konkurrenz werden im Folgenden hinsichtlich ihrer Auswirkungen auf den Artenreichtum diskutiert.

Unter Einbeziehung der in Abschnitt 4.3.2 erhaltenen Ergebnisse soll die Frage geklärt werden, welchen Einfluss biotische Parameter auf den Gesamtartenreichtum haben und ob sich daraus Indizien für eine interspezifische Konkurrenz ableiten lassen.

\section{Interspezifische Konkurrenz}

Nach ZoBel (1992) kommt es in einer Pflanzengesellschaft zu einem ,asymmetrischen Wettbewerb" mit Konkurrenzausschluss als Folge, wenn der Einfluss einer konkurrenzstärkeren Art auf die schwächere zu groß wird. Dies könne im Laufe der Zeit zu einer Reduzierung des Artenreichtums führen, wenn einige Arten einen höheren Anteil an 
verfügbaren Ressourcen für sich beanspruchen. Aufgrund der günstigeren Wachstumsbedingungen erreichen einige Arten eine größere Wuchshöhe, wodurch es zu Lichtkonkurrenz komme. ZOBEL führt veränderte Lebensraumbedingungen wie Stickstoffeinträge als Auslöser dieser asymmetrischen Bedingungen an. Auch ein Wandel in der Zusammensetzung eines Artenpools werde vor allem über Lichtkonkurrenz bestimmt. Ebenso führt НовОНM (1998: 36 f.) die Lichtkonkurrenz als reduzierenden Faktor der Artenvielfalt an. Nach ihm steigen Artenzahlen bis zu einem mittleren Nährstoffniveau an und sinken bei Überschreitung dieser Schwelle wieder $a b$, da durch verstärktes Wachstum einiger Arten das Licht zum begrenzenden Faktor wird. Dem entgegen wirken laut ZOBEL (1992) sämtliche Maßnahmen, welche die vertikale Heterogenität einer Pflanzengesellschaft und dadurch die Lichtkonkurrenz minimieren. Dies könnte zum einen durch die in Abschnitt 5.4.1 erwähnten natürlichen Störungen und zum anderen beispielsweise durch Störungen von Weidetieren (VAN DER MAAREL \& SYKES 1993) oder menschliche Einflüsse wie Mahd oder das Roden von Bäumen und Sträuchern (HUBER 1994) gewährleistet werden.

Die eigenen Untersuchungen lassen hingegen keine Indizien für eine interspezifische Konkurrenz zwischen Gefäßpflanzenarten in den Trockenrasengesellschaften erkennen (vgl. Tab. 39). Der Gefäßpflanzenartenreichtum steigt linear mit der Deckung der Gefäßpflanzen an. Weder Licht- noch Wurzelkonkurrenz scheinen eine Rolle zu spielen. Studien an nordostdeutschen (DENGLER 2004c) sowie öländischen Trockenrasen (LÖBEL 2002: 161) kamen zu gleichen Resultaten.

In zahlreichen Studien (z. B. LÖBEL 2002, DENGLER 2004c, LÖBEL et al. 2004), ebenso in der eigenen, konnte der negative Einfluss einer dichter geschlossenen Gefäßpflanzenschicht auf den Flechtenartenreichtum nachgewiesen werden, was vermutlich auf die Lichtkonkurrenz zurückzuführen sein könne. Ebenso ist die Deckung der Flechtenschicht negativ mit den Gefäßpflanzenartenzahlen korreliert. Dies könnte dadurch erklärt werden, dass Flechten selbst an Extremstandorten, an welchen nur noch wenige Gefäßpflanzenarten vorkommen können, gut gedeihen und dichte Rasen zu bilden vermögen. RUNDEL (1978) bzw. FRAHM et al. (2000) konnten zudem eine keimungshemmende Wirkungen von Flechtensubstanzen auf Diasporen von Gefäßpflanzen feststellen. Es wäre also denkbar, dass einige der von den epigäischen Flechten produzierten Substanzen ähnliche Auswirkungen haben.

Nach FRAHM (2001: 180) kommen sowohl fördernde als auch beeinträchtigende Wirkungen zwischen Moosen und Gefäßpflanzen vor (z B. keimungsfördernde oder -hemmende Wirkung von Moosen auf Diasporen). Er führt beispielsweise die Konkurrenz um den zur Verfügung stehenden Raum an. Nach FRAHM et al. (2000) ist ebenso eine Konkurrenz zwischen Moosen und Flechten festzustellen. Flechten seinen dabei durch die Produktion von Substanzen, welche die Keimung von Moossporen hemmen im Vorteil. Zudem können laut FrAHM (2001: 180) Strauchflechten in Heiden und Tundren Moose verdrängen. Hinsichtlich der eigenen Ergebnisse lässt sich jedoch keine dieser Annahmen bestätigen. Der Moosartenreichtum in den Trockenrasen auf Saaremaa ist nicht signifikant mit den Deckungsgraden der übrigen Artengruppen korreliert. Nach LÖBEL (2002: 161) ist auf Öland eine leichte Abnahme und laut DENGLER (2004c) in Nordostdeutschland sogar eine leichte Zunahme der Moosartendichten bei steigenden Deckungsgraden der Gefäßpflanzen zu verzeichnen. Die leichte Zunahme ist nach Vermutung des letztgenannten Autors auf das feuchtere Mikroklima in dichter geschlossenen Beständen zurückzuführen.

Die Ergebnisse dieser Arbeit weisen darauf hin, dass abiotische Parameter, die einen Ausschluss von konkurrenzstarken Gefäßpflanzenarten bedingen, die Artenvielfalt von Moosen und Flechten begünstigen. 


\subsubsection{Historische Parameter}

\section{Landhebungsprozesse}

Untersuchungen von PÄRTEL \& ZOBEL (1999) in den westestnischen Alvaren ergaben einen signifikant positiven Zusammenhang zwischen der Meereshöhe und der Größe des regionalen Artenpools sowie der Artendichte auf kleinen Flächen $\left(0,04 \mathrm{~m}^{2}\right)$. Dies führen sie auf die Gegebenheit zurück, dass höher gelegene Gebiete im Zuge des Landhebungsprozesses zuerst aus dem Meer erschienen (vgl. Abschnitt 2.2) und somit länger Zeit hatten besiedelt zu werden.

Die eigenen Untersuchungen konnten eine positive Auswirkung auf die Artendichten in den $4 \mathrm{~m}^{2}$ großen Flächen jedoch nicht bestätigen. Die einfachen Regressionsanalysen ergaben einen unimodalen Zusammenhang bezogen auf die Gesamt- und Gefäßpflanzenartendichte. Das Ergebnis sollte jedoch nicht überinterpretiert werden, da die Aufnahmeflächen im Untersuchungsgebiet sehr ungleich verteilt sind, wodurch sich dieser Effekt ergeben haben könnte.

\section{Anthropozoogene Einflüsse}

PÄRTEL \& ZOBEL (1999) nehmen an, dass die Zusammensetzung eines lokalen Artenpools entscheidend durch die langzeitlichen anthropogenen Einwirkungen und durch spezielle Ereignisse (z. B. Feuer) beeinflusst wird. Untersuchungen von DUPRÉ \& DIEKMANN (2001) in Südschweden konnten verdeutlichen, dass die Artenzusammensetzung beweideter von jener unbeweideter Flächen abweicht. Die Autoren konnten außerdem aufzeigen, dass extensiv beweidete Flächen im Allgemeinen artenreicher sind als unbeweidete. Bei intensiver Beweidung zeige sich jedoch ein Rückgang der Artendichten. Dies spricht für die bereits erwähnte ,intermediate disturbance hypothesis“ nach CONNELL (1978).

Der Beweidung wird ein weiterer positiver Einfluss auf die Artenvielfalt eines Vegetationstyps durch Zoochorie zugeschrieben. Nach POSCHLOD et al. (2004) sind Weidetiere (neben dem Menschen) sogar der wichtigste Faktor für eine Verbreitung von Arten über weite Strecken. Die Alvargebiete Saaremaas wurden seit jeher beweidet (z. B. POSKA \& SAARSE 2002), was nach PÄRTEL et al. (1999b) einen positiven Effekt auf die Artenvielfalt in den Trockenrasen hat.

\subsubsection{Zusammenfassung der Phytodiversitätsmuster}

Um die Frage zu klären, welchen Einfluss die abiotischen Parameter auf den Artenreichtum haben, und ob der Artenreichtum der Artengruppen in unterschiedlicher Weise beeinflusst wird, habe ich einfache und multiple Regressionsanalysen durchgeführt (vgl. 4.3.1). Die abiotischen Parameter, welche in die jeweiligen multiplen Regressionsmodelle aufgenommen wurden, erklären 42,3 \% der Varianz der Gesamtartendichte (Tab. 34), 48,2 \% der Gefäßpflanzenartendichte (Tab. 35), 47,9\% der Moosartendichte (Tab. 36) und 37,8\% der Flechtenartendichte (Tab. 37) auf $4 \mathrm{~m}^{2}$. Bezogen auf die Gesamt-, Moos- sowie Flechtenartendichte stellt die Überstauung, hinsichtlich der Gefäßpflanzenartendichte der Gesteinsanteil des Bodens den einflussreichsten Parameter dar.

Zur Klärung der Frage, welchen Einfluss die biotischen Parameter auf den Gesamtartenreichtum haben, und ob sich daraus Indizien für eine interspezifische Konkurrenz ableiten lassen, habe ich die Residuen der multiplen Regressionsanalysen mit den Deckungsgraden der Artengruppen korreliert (Abschnitt 4.3.2). Interspezifische Konkurrenzbeziehungen konnten ausschließlich zwischen Gefäßpflanzen und Flechten festgestellt werden, während Gefäßpflanzen oder Flechten jeweils untereinander durch linear positive 
Beziehungen gekennzeichnet sind. Hinsichtlich der Moose sind keine Zusammenhänge erkennbar.

Die Ergebnisse stimmen vorwiegend mit jenen der vorliegenden Literatur überein. Bezogen auf die Instabilität des Bodens (durch Frosthebung), die Größe der Trockenrasengebiete sowie die Höhe ü. NN und deren Auswirkungen auf die Artendichten weichen die Ergebnisse leicht von jenen aus der Literatur ab. 


\section{Zusammenfassung}

Ziel dieser Diplomarbeit war es, sämtliche auf der estnischen Ostseeinsel Saaremaa vorkommende Trockenrasengesellschaften floristisch und ökologisch zu charakterisieren, sie in ein überregionales pflanzensoziologisches System einzuordnen und ihren Artenreichtum vergleichend zu bewerten. Im Zeitraum vom 29.05. bis 14.08.2004 habe ich zu diesem Zweck 231 Vegetationsaufnahmen nach einem modifizierten Braun-Blanquet-Ansatz auf einheitlich $4 \mathrm{~m}^{2}$ großen, repräsentativ über die Trockenrasengebiete der Insel verteilten Flächen angefertigt. Dabei habe ich sämtliche Gefäßpflanzen, Moose und Flechten einschließlich epigäischer, epilithischer, epiphytischer und epixyler Sippen erfasst. Für jede Aufnahme habe ich die prozentualen Deckungsgrade der Vegetation (getrennt nach Schichten und Artengruppen) sowie der abgestorbenen Pflanzenteile und freiliegenden Gesteine geschätzt. Außerdem wurden das Mikrorelief und die Tiefgründigkeit der Böden gemessen. Der Humusgehalt, die Bodenart, die Kationenaustauschkapazität und Basensättigung, der $\mathrm{pH}-$ Wert und der Kalkgehalt des durchwurzelten Oberbodens $(0-10 \mathrm{~cm})$ wurden im Labor bestimmt. Anhand topografischer Karten des Gebietes habe ich die Höhe über dem Meeresspiegel ermittelt und mittels Luftbildaufnahmen die Größe der einzelnen Trockenrasengebiete errechnet. Ich konnte sieben Trockenrasengesellschaften auf Assoziationsebene unterscheiden. Davon ließen sich sechs bekannten Assoziationen mit formal gültigen Syntaxonnamen zuordnen, die Stellung einer Einheit ist bislang noch nicht geklärt. Sie werden jeweils mit einer Vegetationstabelle präsentiert. Die Trockenrasengesellschaften verteilen sich auf die drei Hauptgruppen Koelerio-Corynephorenea (4 Assoziationen), Sedo-Scleranthenea (2 Assoziationen) und Festuco-Brometea (1 Gesellschaft). Die floristischen Unterschiede der Hauptgruppen sowie der Gesellschaften sind durch eine Stetigkeitstabelle illustriert. Die Trockenrasenbestände der Unterklasse Koelerio-Corynephorenea wachsen auf tiefgründigen Sandböden, die hinsichtlich der Wasserund Nährstoffversorgung die extremsten Standorte darstellen. Bestände der Unterklasse SedoScleranthenea kommen im Untersuchungsgebiet auf Alvarflächen und häufig auf synanthropen Standorten außerhalb der Alvare vor. Sie besiedeln flachgründige, humus- und skelettreiche Böden. Die basiphilen Halbtrockenrasen der Klasse Festuco-Brometea wachsen auf Böden mit den relativ günstigsten Wasser- und Nährstoffverhältnissen. Die mittlere Artendichte auf $4 \mathrm{~m}^{2}$ variiert zwischen 1 und 71 (Ø 35). Sie ist in den Sedo-Scleranthenea am höchsten (Ø 42,6), gefolgt von den Festuco-Brometea (Ø 40,5) und am niedrigsten in den Koelerio-Corynephorenea (Ø 24,3). Insgesamt ist das Crepido-Allietum die artenreichste (Ø 47,7), das Caricetum arenariae die artenärmste (Ø 14,7) Assoziation. Der prozentuale Anteil der Gefäßpflanzen beträgt 6-100\%, jener der Moose 0-48 \% sowie jener der Flechten 0$78 \%$. In den Festuco-Brometea ist der Anteil der Gefäßpflanzen (Ø $75 \%$ ) am höchsten, in den Sedo-Scleranthenea jener der Moose (Ø $28 \%$ ) und in den Koelerio-Corynephorenea jener der Flechten (Ø $31 \%$ ). Ich habe einfache lineare sowie multiple Regressionsanalysen verwendet, um die Einflüsse der Umweltbedingungen, der Landschaftsstruktur und der biotischen Wechselwirkungen auf die gesamte und gruppenspezifische Phytodiversität auszuwerten. Die abiotischen Parameter, welche in die jeweiligen multiplen Regressionsmodelle aufgenommen wurden, erklären 42,3 \% der Varianz der Gesamtartendichte, 48,2 \% der Gefäßpflanzenartendichte, 47,9\% der Moosartendichte und 37,8 \% der Flechtenartendichte auf $4 \mathrm{~m}^{2}$. Bezogen auf die Gesamt-, Moos- sowie Flechtenartendichte stellt die Überstauung, hinsichtlich der Gefäßpflanzenartendichte der Gesteinsanteil des Bodens den einflussreichsten Parameter dar. Interspezifische Konkurrenzbeziehungen konnten ausschließlich zwischen Gefäßpflanzen und Flechten festgestellt werden, während Gefäßpflanzen bzw. Flechten jeweils untereinander durch linear positive Beziehungen gekennzeichnet sind. Hinsichtlich der Moose sind keine signifikanten Zusammenhänge ersichtlich. Zahlreiche der im Untersuchungsgebiet vorkommenden Trockenrasenbestände werden im Anhang I der FFHRichtlinie als schützenswert ausgewiesen. Insgesamt konnte ich vier neue Arten für Estland nachweisen. Drei davon sicher, bei einer ist die taxonomische Zuordnung der gesammelten Pflanzen noch nicht abschließend geklärt. 


\section{Quellen}

Ackermann, W., Durka, W. (1998): SORT 4.0 - Programm zur Bearbeitung von Vegetationsaufnahmen und Artenlisten - Handbuch. - 138 S., Mskr., München [u. a.].

Albertson, N. (1950): Das grosse südliche Alvar der Insel Öland - Eine pflanzensoziologische Übersicht. Sven. Bot. Tidskr. 44: 270-331, 4 Tafeln.

Andersson, S. (1990): A phenetic study of Crepis tectorum in Fennoscandia and Estonia. - Nordic J. Bot. 9: 589-600, Copenhagen.

Anonymus (1970): Eesti NSV kliimaatlas. - 209 S., Tallinn.

Barot, S., Gignoux, J. (2004): Mechanisms promoting plant coexistence: can all the proposed processes be reconciled? Oikos, 106 (1):185-192.

Begon, M., Harper, J. L., Townsend, C. R. (1998): Ökologie. - Sauer, K. P. [Hrsg.], 750 S., Spektrum, Akad. Verl., Heidelberg, Berlin.

Bergmeier, E., Härdtle, W., Mierwald, U., Nowak, B., Peppler, C. (1990): Vorschläge zur syntaxonomischen Arbeitsweise in der Pflanzensoziologie. - Kiel. Not. Pflanzenkd. Schleswig-Holstein Hamb. 20: 92-110.

Blom, H. H. (1996): A revision of the Schistidium apocarpum complex in Norway and Sweden. - Bryophytorum Bibl. 49: 333 S., Cramer, Berlin [u. a.].

Blum, W. E. H. (1992): Bodenkunde in Stichworten. - Schroeder, D. [Begr.]: 5. Aufl., 175 S., Hirt, Berlin.

Bohn, U., Gollub, G., Hettwer, C. (2000): Karte der natürlichen Vegetation Europas - Maßstab 1:2 500000 Legende. - 153 S., Bundesamt für Naturschutz, Bonn.

Bohn, U., Gollub, G., Hettwer, C., Neuhäuslová, Z., Schlüter, H., Weber, H. (2003) [Hrsg.]: Karte der natürlichen Vegetation Europas - Maßstab 1 : 2500000 - Erläuterungstext. - 655 S., 13 Kt., CD-ROM, Bundesamt für Naturschutz, Bonn.

Bohn, U., Gollub, G., Hettwer, C., Neuhäuslová, Z., Raus, T., Schlüter, H., Weber, H., Hennekens, S. (2004) [Hrsg.]: Karte der natürlichen Vegetation Europas - Maßstab 1 : 2500000 - Erläuterungstext, Legende, Karten [dt./engl.]. - CD-ROM + 19-seitiges Booklet, Bundesamt für Naturschutz, Bonn.

Bohn, U., Neuhäusl, R. (2000) [Hrsg.]: Karte der natürlichen Vegetation Europas - Maßstab 1:2 500000 Karten. - 10 Kt., 1 Legende, Bundesamt für Naturschutz, Bonn.

Braun-Blanquet, J. (1951): Pflanzensoziologie - Grundzüge der Vegetationskunde. - 2. Aufl., XI + 631 S., Springer, Wien.

Brodo, I. M., Sharnoff, S. D., Sharnoff, S. (2001): Lichens of North America. - 795 S., Yale University Press, New Haven \& London.

Brown, I. C. (1943): A rapid method of determining exchangeable hydrogen and total exchangeable bases of soils. - Soil Science 56: 353-357.

Chytrý, M., Tichý, L., Roleček, J. (2003): Local and Regional Patterns of Species Richness in Central European Vegetation Types along the pH/Calcium Gradient. - Folia Geobot. 38: 429-442.

Connell, J. H. (1978): Diversity in tropical rain forests and coral reefs. - Science 199: 1302-1310.

Corley, M. F. V., Crundwell, A. C., Düll, R., Hilland, M. O., Smith, A. J. E. (1981): Mosses of Europe and the Azores - J. Bryol. 16: 337-356.

Corley, M. F. V., Crundwell, A. C. (1991): Additions and amendments to the mosses of Europe and the Azores; an annotated list of species, with synonyms from the recent literature.- J. Bryol. 11: 609-689.

Culberson, C. F., Ammann, K. (1979): Standardmethode zur Dünnschichtchromatographie von Flechtensubstanzen. - Herzogia 5: 1-24.

Damsholt, K. (2002): Illustrated Flora of Nordic Liverworts and Hornworts. - 840 S., Oikos Editorial Office, Lund.

Dengler, J. (1998): Neues von den schmalblättrigen Schwingel-Sippen (Festuca ovina agg. und F. rubra agg.) in Deutschland mit besonderer Berücksichtigung von Schleswig-Holstein und Hamburg. - Kiel. Not. Pflanzenkd. Schleswig-Holstein Hamb. 25/26: 6-32.

Dengler, J. (2001b): Koelerio-Corynephoretea. - Berg, C., Dengler, J., Abdank, A. [Hrsg.]: Die Pflanzengesellschaften Mecklenburg-Vorpommerns und ihre Gefährdung - Tabellenband: 118-136, Weissdorn, Jena.

Dengler, J. (2001c): Festuco-Brometea. - Berg, C., Dengler, J., Abdank, A. [Hrsg.]: Die Pflanzengesellschaften Mecklenburg-Vorpommerns und ihre Gefährdung - Tabellenband: 137-143, Weissdorn, Jena.

Dengler, J. (2003): Entwicklung und Bewertung neuer Ansätze in der Pflanzensoziologie unter besonderer Berücksichtigung der Vegetationsklassifikation. - Arch. Naturwiss. Diss. 14: 297 S., Galunder, Nümbrecht.

Dengler, J. (2004a): Klasse: Koelerio-Corynephoretea Klika in Klika \& V. Novák 1941 - Sandtrockenrasen und Felsgrusfluren von der submeridionalen bis zur borealen Zone. - Berg, C., Dengler, J., Abdank, A., Isermann, M. [Hrsg.]: Die Pflanzengesellschaften Mecklenburg-Vorpommerns und ihre Gefährdung Textband: 301-326, Weissdorn, Jena.

Dengler, J. (2004b): Klasse: Festuco-Brometea Br.-Bl. \& Tx. ex Klika \& Hadač 1944 - Basiphile Magerrasen und Steppen im Bereich der submeridionalen und temperaten Zone. - Berg, C., Dengler, J., Abdank, A., Isermann, M. [Hrsg.]: Die Pflanzengesellschaften Mecklenburg-Vorpommerns und ihre Gefährdung Textband: 327-335, Weissdorn, Jena. 
Dengler, J. (2004c): Phytodiversitätsmuster in nordostdeutschen Trockenrasen. - Kiel. Not. Pflanzenkd. Schleswig-Holstein Hamb. 32: 14-19.

Dengler, J. (2005): Zwischen Estland und Portugal - Gemeinsamkeiten und Unterschiede der Phytodiversitätsmuster europäischer Trockenrasen. - Tuexenia 25: 387-405.

Dengler, J., Löbel, S. (2006): The basiphilous dry grasslands of shallow, skeletal soils (Alysso-Sedetalia) on the island of Öland (Sweden), in the context of North and Central Europe. - Phytocoenologia, Berlin, Stuttgart.

Dengler, J., Berg, C., Eisenberg, M., Isermann, M., Jansen, F., Koska, I., Löbel, S., Manthey, M., Päzolt, J., Spangenberg, A., Timmermann, T., Wollert, H. (2003): New descriptions and typifications of syntaxa within the project 'Plant communities of Mecklenburg-Vorpommern and their vulnerability' - Part I. - Feddes Repert. 114: 587-631.

Dierschke, H. (1994): Pflanzensoziologie - Grundlagen und Methoden. - 683 S., Ulmer, Stuttgart.

Dierßen, K. (1990): Einführung in die Pflanzensoziologie (Vegetationskunde). - 241 S., Wissenschaftliche Buchgesellschaft, Darmstadt.

Dierßen, K. (1996): Vegetation Nordeuropas. - 838 S., Ulmer, Stuttgart.

Doll, R.(1988): Bestimmungsschlüssel für die Carex-Arten des Flachlandes der DDR nach vegetativen und Samen-Merkmalen. - Arch. Freunde Naturg. Mecklb. 28: 80-97.

Dolnik, C. (2003): Artenzahl-Areal-Beziehungen von Wald- und Offenlandgesellschaften - Ein Beitrag zur Erfassung der botanischen Artenvielfalt unter besonderer Berücksichtigung der Flechten und Moose am Beispiel des Nationalparks Kurischen Nehrung (Russland). - Mitt. Arbeitsgem. Geobot. Schleswig-Holstein und Hamburg 62: 183 S., Kiel.

Dupré, C., Diekmann, M. (2001): Differences in species richness and life-history traits between grazed and abandoned grasslands in southern Sweden. - Ecography 24: 275-286.

Dupré, C., Wessberg, C., Diekmann, M. (2002): Species richness in decidous forests: Effects of species pools and environmental variables. - J. Veg. Sci. 13: 505-516.

Eeconet Action Fund (2005) [Hrsg.]: EAF Projects - Estonia. - URL: http://www.eeconet.org/eaf/projects/index.html (Stand: 01.07.05).

Eichwald, K., Kask, M., Kuusk, V., $\quad$ Laasimer, L., $\quad$ Lellep, E., $\quad$ Peikel, E., $\quad$ Rebassoo, H., Remmel, A., Süvalepp, A., Talts, S., Võsamäe, H., Üksip, A. (1978): Eesti NSV Floora VI. - 613 S., Valgus, Tallinn.

Eichwald, K., Kukk, E., Kuusk, V., Lellep, E., Mäemets, A., Rebassoo, H., Sander, R., Talts, S., Trei, T., Viljasoo, L. (1984): Eesti NSV Floora IX. - 448 S., Valgus, Tallinn.

Eilart, J. (1969): The Development of Vegetation from the Aspect of Analysis of Floral Elements. - Laasimer [Hrsg.]: Plant Taxonomy, Geography and Ecology in the Estonian S. S. R.: 17-23, Valgus, Tallinn.

Eisele, W., Zähringer, E. (1997): Vorläufiger Schlüssel zur Bestimmung der Seggen (Carex spec.) BadenWürttembergs nach vegetativen Merkmalen. - Flor. Rundbr. Beih. 5: 26 S., Verlag E. Golze, Göttingen.

Ellenberg, H., Weber, H. E., Düll, R., Wirth, V., Werner, W., Paulißen, D. (1991): Zeigerwerte von Pflanzen in Mitteleuropa. - Scr. Geobot. 18: 248 S., Goltze, Göttingen.

European Commission (2003a) [Hrsg.]: Directive 92/43 EEC - ANHANG I - Natürliche Lebensraumtypen von gemeinschaftlichem Interesse, für deren Erhaltung besondere Schutzgebiete ausgewiesen werden müssen. $12 \mathrm{~S}$., Brussels. URL: http:/www.europa.eu.int/comm/environment/nature/nature_conservation/ eu_enlargement/2004/habitats/annexi_de.pdf (Stand: 24.10.05).

European Commission (2003b) [Hrsg.]: Interpretation Manual of European Union Habitats - EUR25. European Commission,127 S., Brussels.

Ewald, J. (2003): The calcareous riddle: why are there so many calciphilous species in the Central European flora? Folia Geobot. 38: 357-366.

Fischer, P. (2003): Trockenrasen des Biosphärenreservates „Flußlandschaft Elbe“ - Vegetation, Ökologie und Naturschutz. - Arch. Naturwiss. Diss. 15: V + 286 S., CD-ROM, Nümbrecht.

Frahm, J.-P., Reifenrath, K., Specht, A., León Vargas, Y. (2000): Allelopathic effects of crustaceous lichens on the germination of bryophytes and vascular plants. Nova Hedwigia 70: 245-254.

Frahm, J.-P. (2001): Biologie der Moose. - 357 S., Spektrum, Akad. Verl., Heidelberg [u. a.].

Frahm, J.-P., Frey, W. (1992): Moosflora. - UTB 1250: 3. Aufl., 528 S., Ulmer, Stuttgart.

Frahm, J.-P., Frey, W. (2004): Moosflora. - UTB 1250: 4. Aufl., 538 S., Ulmer, Stuttgart.

Frey, W., Lösch, R. (2004): Lehrbuch der Geobotanik - Pflanze und Vegetation in Raum und Zeit. - 2. Aufl., 528 S., Elsevier GmbH, Spektrum, Akad. Verl., München.

Grace, J. B. (1999): The factors controlling species density in herbaceous plant communities: an assessment. Perspectives Plant Ecol. Evol. Syst. 2: 1-28.

Grime, J. P. (1973): Control of Species Density in Herbaceous Vegetation. - J. Environ. Management 1: 151167.

Grime, J. P. (2001): Plant strategies, vegetation processes, and ecosystem properties. - 2nd. ed. Wiley, Chichester, UK.

Grolle, R., Long, D. G. (2000): An annotated check-list of the Hepaticae and Anthocerotatae of Europe and Macaronesia. - J. Bryol. 22: 103-140.

Grubb, P. J. (1977): The maintenance of species-richnes in plant communities: the importance of the regeneration niche. - Biol. Rev. Camb. Philos. Soc. 52: 107-145. 
Hallberg, H. P. (1971): Vegetation auf den Schalenablagerungen in Bohuslän, Schweden. - Acta Phytogeogr. Suec. 56: 136 S., Anhang, Sven. Växtgeogr. Sällsk., Uppsala.

Helm, A., Hanski, I., Pärtel, M. (im Druck): Slow response of plant species richness to habitat loss and fragmentation. - Ecol. Lett.

Herk, K. van, Aptroot, A. (2004): Veldgids korstmossen. - 423 S., KNNV Uitgeverij, Utrecht.

Hobohm, C. (1998): Pflanzensoziologie und die Erforschung der Artenvielfalt - Überarbeitete und erweiterte Fassung der an der Universität Lüneburg eingereichten und angenommenen Habilitationsschrift. - Arch. Naturwiss. Diss. 5: 231 S., Galunder, Wiehl.

Hobohm, C. (2000): Biodiversität. - 214 S., Quelle \& Meyer, Wiebelsheim.

Hobohm, C. (2005): Die Erforschung der Artenvielfalt in Pflanzengesellschaften - eine Zwischenbilanz. Tuexenia 25: 7-28, Göttingen.

Huber, R. (1994): Changes in plant species richness in a calcareous grassland following changes in environmental conditions. - Folia Geobot. Phytotax. 29: 469-482.

Jäger, E. J., Werner, K. (2001) [,„2002”] [Hrsg.]: Gefäßpflanzen: Kritischer Band. - Rothmaler, W. [Begr.]: Exkursionsflora von Deutschland 4: 9. Aufl., 948 S., Spektrum Akademischer Verl., Heidelberg [u. a.].

Jermacāne, S., Laiviņš, M. (2001): Dry calcareous dolomite outcrop and grassland and communities on the Daugava River Bank near Dzelmes. - Latvijas Veǵetācija 4: 51-69.

Jõgi, J. (2002): Agro- ja Mikrokliima. - Kään, H., Mardiste, H.,Nelis, R., Pesti, O. [Hrsg.]: Saaremaa. Loodus. Aeg. Inimene. (1. Osa): 68-70, Tallinna Raamatutrüikoda, Tallinn.

Jonsell, B., Karlsson, T. (2004): Endemic vascular plants in Norden. - Jonsell, B. [Hrsg.]: Flora Nordica General Volume: 139-159, Royal Swedish Acad. of Sciences, Stockholm.

Kannukene, L. (2002): Sammaltaimed. - Kään, H., Mardiste, H.,Nelis, R., Pesti, O. [Hrsg.]: Saaremaa. Loodus. Aeg. Inimene. (1. Osa): 95, Tallinna Raamatutrüikoda, Tallinn.

Korneck, D. (1974): Xerothermvegetation von Rheinland-Pfalz und Nachbargebieten. - Schriftenr. Vegetationskd. 7: 196 S., 158 Tab., Bundesforschungsanstalt für Naturschutz und Landschaftsökologie, Bonn.

Korneck, D. (1975): Beitrag zur Kenntnis mitteleuropäischer Felsgrus-Gesellschaften (Sedo-Scleranthetalia). Mitt. Florist.-Soziol. Arbeitsgem. N. F. 18: 45-102, 4 Tab.

Korneck, D., Schnittler, M., Klingenstein, F., Ludwig, G., Takla, M., Bohn, U., May, R. (1998): Warum verarmt unsere Flora? - Auswertung der Roten Liste der Farn- und Blütenpflanzen Deutschlands. - Klingenstein, F., Ludwig, G. [Hrsg.]: Ursachen des Artenrückgangs von Wildpflanzen und Möglichkeiten zur Erhaltung der Artenvielfalt. - Schriftenr. Vegetationskd. 29: 299-444, Bundesamt für Naturschutz.

Krausch, H.-D. (1962): Vorschläge zur Gliederung der mitteleuropäischen Sand- und Silikat-Trockenrasen. Mitt. Florist.-Soziol. Arbeitsgem. N. F. 9: 266-269.

Krauss, J., Klein, A.-M., Steffan-Dewenter, I., Tscharntke, T. (2004): Effects of habitat area, isolation, and landscape diversity on plant species richness of calcareous grasslands. - Biodiversity and Conservation 13: $1427-1439$.

Kull, T., Kukk, T., Leht, M., Krall, H., Kukk, Ü., Kull, K., Kuusk, V. (2002): Distribution trends of rare vascular plant species in Estonia. - Biodiversity and Conservation 11: 171-196.

Laasimer, L. (1965): Eesti NSV Taimkate. - 397 S., Valgus, Tallinn.

Laasimer, L. (1969): Vegetation Mapping and Division Applied in Planning. - Laasimer [Hrsg.]: Plant Taxonomy, Geography and Ecology in the Estonian S. S. R.: 66-70, Valgus, Tallinn.

Laasimer, L. (1975): Die Alvare und die Alvarwälder in Estland und ihr Schutz. - Kumari, E., Hang, V., Mäemets, A., Renno, O. [Hrsg.]: Eesti Loodus-Harulduste Kaitseks: 90-102, Valgus, Tallin.

Lang, G., Rybníček, K., Rybníčková, E. (2003): Spätglaziale und holozäne Vegetationsgeschichte Europas. Bohn, U., Gollub, G., Hettwer, C., Neuhäuslová, Z., Schlüter, H., Weber, H. [Hrsg.]: Karte der natürlichen Vegetation Europas - Maßstab 1 : 2500000 - Erläuterungstext: S. 87-104, CD-ROM, Bundesamt für Naturschutz, Bonn.

Leht, M. [Hrsg.] (1999): Eesti taimede määraja. EPMÜ ZBI, Eesti Loodusfoto, 447 S., Greif, Tartu.

Lippmaa, T. (1933): Taimeühingute Uurimise Metoodika Ja Eesti Taimeühingute Klassifikatsiooni Põhijooni Referat: Grundzüge der pflanzensoziologischen Methodik nebst einer Klassifikation der Pflanzenassoziationen Estlands. - Acta Inst. et Horti Bot. Univ. Tart. 3: 141-159.

Lippmaa, T. (1935): Eesti Geobotaanika Põhijooni. [Avec un résumé: Aaperçu Géobotanique de l'Estonie.] Acta 4: $1-151$.

Löbel, S. (2002): Trockenrasen auf Öland: Syntaxonomie - Ökologie - Biodiversität. - $178+$ XIX S., Beilagetabellen, unpubl. Diplomarb., Inst. für Ökol. und Umweltchem., Univ. Lüneburg.

Löbel, S., Dengler, J., Hobohm, C. (2004): Beziehungen zwischen der Artenvielfalt von Gefäßpflanzen, Moosen und Flechten in Trockenrasen der Insel Öland (Schweden). - Kiel. Not. Pflanzenkd. Schleswig-Holstein Hamb. 32: 9-13.

Löbel, S., Dengler, J., Hobohm, C. (eingereicht): Species richness of vascular plants, bryophytes and lichens in dry grasslands on Öland: The effects of environmental conditions, landscape structure and biotic interactions. - zur Einreichung bei „Journal of Vegetation Science“. 
Lõugas, V. (1988): Steingräberfeld in Võhma (Insel Saaremaa). - Proceedings Estonian Academy of Sciences, Social Sciences 37: 348-351.

Lundholm, J. T., Larson, D. W. (2003): Relationships between spatial environmental heterogeneity and plant species diversity on a limestone pavement. - Ecography 26: 715-722.

Lundqvist, Å. (1986) [Hrsg.]: Ölands kärlväxtlora. - Sterner, R. [Begr.]: 2. Aufl., 400 S., Förlagstjänsten, Stockholm.

Maarel, E. van der, Sykes, M. T. (1993): Small-scale plant species turnover in a limestone grassland: the carousel model and some comments on the niche concept. - J. Veg. Sci. 4: 179-188

Mardiste, H. (2002): Üldandmed. - Kään, H., Mardiste, H.,Nelis, R., Pesti, O. [Hrsg.]: Saaremaa. Loodus. Aeg. Inimene. (1. Osa): 13-19, Tallinna Raamatutrüikoda, Tallinn.

Marksoo, A. (2002): Rahvastik ja asustus. - Kään, H., Mardiste, H.,Nelis, R., Pesti, O. [Hrsg.]: Saaremaa. Loodus. Aeg. Inimene. (1. Osa): 377-466, Tallinna Raamatutrüikoda, Tallinn.

Moore, J. L., Mouquet, N., Lawton, J. H., Loreau, M. (2001): Coexistence, saturation and invasion resistance in simulated plant assemblages. - Oikos 94: 303-314.

Mossberg, B., Stenberg, L. (2003): Den nya nordiska floran [schwed.]. - 928 S., Wahlström \& Widstrand, Stockholm.

Nebel, M., Philippi, G. [Hrsg.] (2000): Die Moose Baden Württembergs - Band 1. Allgemeiner Teil (Bryophytina I, Andreaeales bis Funariales). - 512 S., Ulmer, Stuttgart.

Nebel, M., Philippi, G. [Hrsg.] (2001): Die Moose Baden Württembergs - Band 2. Spezieller Teil (Bryophytina II, Schistostegales bis Hypnobryales). - 529 S., Ulmer, Stuttgart.

Neumann, A. (1952): Vorläufiger Bestimmungsschlüssel für Carex-Arten Nordwestdeutschlands m blütenlosen Zustande. - Mitt. Florist.-Soziol. Arbeitsgem. N. F. 3: 44-77.

Nyholm, E. (1986): Illustrated Flora of Nordic Mosses. Fasc. 1. Fissidentaceae - Seligeriaceae. - 72 S., Copenhagen und Lund.

Nyholm, E. (1989): Illustrated Flora of Nordic Mosses. Fasc. 2. Pottiaceae - Splachnaceae - Schistostegaceae. 141 S., Nordic Bryological Society, Copenhagen und Lund.

Nyholm, E. (1993): Illustrated Flora of Nordic Mosses. Fasc. 3. Bryaceae - Rhodobryaceae - Mniaceae Cinclidiaceae - Plagiomniaceae. - 244 S., Nordic Bryological Society, Copenhagen und Lund.

Nyholm, E. (1998): Illustrated Flora of Nordic Mosses. Fasc. 4. Aulacomniacaeae - Meesiaceae Catoscopiaceae - Bartramiaceae - Timmiaceae - Encalyptaceae - Grimmiaceae - Ptychomitraceae Hedwigiaceae - Orthotrichaceae. - 405 S., Nordic Bryological Society, Copenhagen und Lund.

Oberdorfer, E. (2001): Pflanzensoziologische Exkursionsflora - für Deutschland und angrenzende Gebiete. -8 . Aufl., 1051 S., Ulmer, Stuttgart.

Paal, J. (1998): Rare and threatened plant communities of Estonia. - Biodiversity and Conservation 7: 10271049.

Paal, J. (2000): "Loodusdirektiivi” elupaigatüüpide käsiraamat. Eesti Natura 2000. - 202 S., TÜ botaanika ja ökoloogia instituut Tartu.

Pärtel, M. (2002): Local plant diversity patterns and evolutionary history at the regional scale. - Ecology 83: 2361-2366.

Pärtel, M., Kalamees, R., Zobel, M., Rosén, E. (1999a): Alvar grasslands in Estonia: variation in species composition and community structure. - J. Veg. Sci. 10: 561-568.

Pärtel, M., Mändla, R., Zobel, M. (1999b): Landscape history of a calcareous (alvar) grassland in Hanila, western Estonia, during the last three hundred years. - Landscape Ecol. 14: 187-196.

Pärtel, M., Zobel, M., Zobel, K., Maarel, E. van der (1996): The species pool and its relation to species richness: evidence from Estonian plant communities. - Oikos 75: 111-117.

Pärtel, M., Zobel, M. (1999): Small-scale species richness in calcareous grasslands determined by the species pool, community age and shoot density. - Ecography 22: 153-159.

Paton, J. A. (1999): The Liverwort flora of the British Isles. - 626 S., Harley Books, Martins, Colchester, Essex.

Pfadenhauer, J., Poschlod, P., Buchwald, R. (1986): Überlegungen zu einem Konzept geobotanischer Dauerbeobachtungsflächen für Bayern. Teil I. Methodik der Anlage und Aufnahme. - Ber. ANL 10: 41-60.

Poschlod, P., Tackenberg, O., Bonn, S. (2004): Plant dispersal potential and ist relation to species frequency and co-existence. - Maarel, E. van der [Hrsg.]: Vegetation Ecology: 147-171, Blackwell.

Poschlod, P., Schumacher, W. (1998): Rückgang von Pflanzen und Pflanzengesellschaften des Grünlandes Gefährdungsursachen und Handlungsbedarf. - Klingenstein, F., Ludwig, G. [Hrsg.]: Ursachen des Artenrückgangs von Wildpflanzen und Möglichkeiten zur Erhaltung der Artenvielfalt. - Schriftenr. Vegetationskd. 29: 83-99, Bundesamt für Naturschutz, Bonn.

Poska, A., Saarse, L. (2002): Vegetation development and introduction of agriculture to Saaremaa Island, Estonia: the human response to shore displacement. - The Holocene 12 (5): 555-568.

Pott, R. (1995): Die Pflanzengesellschaften Deutschlands. - 2. Aufl., 622 S., Ulmer, Stuttgart.

Purvis, O. W., Coppins, B. J., Hawkswort, D. L., James, P. W., Moore, D. M. (1992) [Hrsg.]: The Lichen Flora of Great Britain and Ireland. - IX + 710 S., British Lichen Society, London.

Randlane, T. (2002): Samblikud. - Kään, H., Mardiste, H.,Nelis, R., Pesti, O. [Hrsg.]: Saaremaa. Loodus. Aeg. Inimene. (1. Osa): 111-112, Tallinna Raamatutrüikoda, Tallinn. 
Rathmann (2003): The UNDP/GEF Baltic Wind Atlas - The UNDP/GEF Regional Baltic Wind Energy Programme. - 36 S., Risø National Laboratory, Roskilde.

Raudsepp, H., Jaagus, J. (2002): Üldiseloomustus. - Kään, H., Mardiste, H.,Nelis, R., Pesti, O. [Hrsg.]: Saaremaa. Loodus. Aeg. Inimene. (1. Osa): 65-68, Tallinna Raamatutrüikoda, Tallinn.

Raukas, A., Teedumäe, A. (1997): Geology and mineral resources of Estonia. - 436 S., Estonian Academy Publishers, Tallinn.

Reintam, L. (1995): Soils in Estonia. - Boguslawski, E. v., Limberg, P. [Hrsg]: Boden und Düngung: 122-131, Mitt. Intn. Arbeitsgem. Bodenfruchtbarkeit IBG, ISSS, Tartu.

Reisigl, H., Keller, R. (1994): Alpenpflanzen im Lebensraum - Alpine Rasen Schutt und Felsenvegetation. - 2. Aufl., 148 S., Fischer-Verlag, Stuttgart.

Reitalu, M., Roosaluste, E. (2002): Soontaimed. - Kään, H., Mardiste, H.,Nelis, R., Pesti, O. [Hrsg.]: Saaremaa. Loodus. Aeg. Inimene. (1. Osa): 96-99, Tallinna Raamatutrüikoda, Tallinn.

Rosén, E., Borgegård, S.-O. (1999): The open cultural landscape. - Rydin, H., Snoeijs, P., Diekmann, M. [Hrsg.]: Swedish plant geography - Dedicated to Eddy van der Maarel on his $65^{\text {th }}$ birthday. - Acta Phytogeogr. Suec. 84: 5-14.

Rosenzweig, M. L., Abramsky, Z. (1993): How are diversity and produktivity related? - Ricklefs, R. E., Schluter, D. [Hrsg.]: Species diversity in ecological communities: 52-65, University of Chicago Press, Chicago.

Rundel, P. W. (1978): The ecological role of secondary lichen substances. - Biochem. Syst. Ecol. 6: 157-170.

Rūsiņa, S. (2005): Diagnostic species of mesophylous and xerophylous grassland plant communities in Latvia. Latvijas Universitates Raksti 685: 70-95.

Saarse, L., Königsson, L.-K. (1992): Holocene environmental changes on the island of Saaremaa, Estonia. PACT 37: 97-131.

Santesson, R., Moberg, R., Nordin, A., Tønsberg, T., Vitikainen, O. (2004): Lichen-forming and lichenicolous fungi of Fennoscandia. - Museum of Evolution, Uppsala: $359 \mathrm{~S}$.

Schlichting, E., Blume, H.-P., Stahr, K. (1995): Bodenkundliches Praktikum - Eine Einführung in pedologisches Arbeiten für Ökologen, insbesondere Land- und Forstwirte, und für Geowissenschaftler. - Pareys Studientexte 81: 2. Aufl., 295 S., Blackwell, Berlin [u. a.].

Schöller, H. (1997): Ökologie und Verbreitung. - Schöller, H. [Hrsg.]: Flechten: Geschichte, Biologie, Systematik, Ökologie, Naturschutz und kulturelle Bedeutung. Begleitband zur Ausstellung "Flechten Kunstwerke der Natur". Kl. Senckenberg-Reihe 27: 83-109, Senckenberische Naturforschende Gesellschaft, Frankfurt am Main.

Schubert, R., Hilbig, W., Klotz, S. (2001): Bestimmungsbuch der Pflanzengesellschaften Deutschlands. 472 S., Spektrum, Heidelberg [u. a.].

Schulze, E.-D., Beck, E., Müller-Hohenstein, K. (2002): Pflanzenökologie. - 846 S., Spektrum Akademischer Verl., Heidelberg [u. a.].

Sérusiaux, E., P. Diederich, A. M. Brand, Boom, P. van den (1999): New or interesting lichens and lichenicolous fungi from Belgium and Luxembourg. - Lejeunia Nouv. Sér. 162: $95 \mathrm{~S}$.

Smith, A. E. J. (1978): The Moss Flora of Britain and Ireland. - 706 S., Cambridge University Press, Cambridge.

Smith, A. E. J. (1990): The Liverworts of Britain and Ireland. - 362 S., Cambridge University Press, Cambridge.

Suija, A., Nõmm, M., Boch, S. (2005): New Estonian Records - Lichens and lichenicolous fungi. - Folia Cryptog. Estonica, Fasc. 41: 135-136.

Teras, T. (2002): Mullastik. - Kään, H., Mardiste, H.,Nelis, R., Pesti, O. [Hrsg.]: Saaremaa. Loodus. Aeg. Inimene. (1. Osa): 127-136, Tallinna Raamatutrüikoda, Tallinn.

Tomson, A. (1937): La végétation de la presqu'île de Sõrve (Estonie) [estn., franz. Zus.]. - Eesti Loodusteaduse Arh., II Seeria, 16(1/2): 1-84, 1 Kt.

Trass, H. (2002): Taimkatte Rajoneerimine. - Kään, H., Mardiste, H.,Nelis, R., Pesti, O. [Hrsg.]: Saaremaa. Loodus. Aeg. Inimene. (1. Osa): 126, Tallinna Raamatutrüikoda, Tallinn.

Tutin, T. G., Heywood, V. H., Burges, N. A., Valentine, D. H., Walters, S. M., Webb, D. A. (1976) [Hrsg.]: Flora Europaea - Volume 4: Plantaginaceae to Compositae (and Rubiaceae). - XXIX + 505 S., 5 Kt., Cambridge University Pr., Cambridge.

Tutin, T. G., Heywood, V. H., Burges, N. A., Valentine, D. H., Walters, S. M., Webb, D. A. (1980) [Hrsg.]: Flora Europaea - Volume 5: Alismataceae to Orchidaceae (Monocotyledones). - XXXVI + 452 S., 5 Kt., Cambridge University Pr., Cambridge.

Tyler, G. (1996): Soil chemistry and plant distibutions in rock habitats of southern Sweden. - Nord. J. Bot. 16: $609-635$.

Veski, S. (1996): History of Vegetation and Human Impact in Northern Saaremaa, Estonia based on the Biostratigraphy of the Surusoo Mire: Preliminary Results. - PACT 51: 57-66.

Veski, S. (1998): Vegetation History, Human Impact and Palaeogeography of West Estonia. Pollen Analytical Studies of Lake and Bog Sediments. - Striae 38: 119 S., Societas Upsaliensis Pro Geologia Quarternaria, Uppsala. 
Vilberg, G. (1927): Die Alvare und die Alvarvegetation in Ost-Harrien [in Estnisch, mit deutscher Zusammenfassung]. - Tartu Ülikooli Juures Oleva Loodus. Seltsi Aruaded 34: 11-139, 4 Tafeln.

Waide, R. B., Willig, M. R., Steiner, C. F., Mittelbach, G., Gough, L., Dodson, S. I., Juday, G. P., Parmenter, R. (1999): The Relationship Between Productivity and Species Richness. - Ann. Rev. Ecol. Syst. 30: 257-300.

Wild, A. (1995): Umweltorientierte Bodenkunde - Eine Einführung. - 328 S., Spektrum, Akad. Verl., Heidelberg, Berlin, Oxford.

Wilmanns, O. (1993): Ökologische Pflanzensoziologie. - 5. Aufl., 479 S., Quelle \& Meyer, Heidelberg.

Wirth, V. (1995): Flechtenflora. - UTB 1062: 2. Aufl., 661 S., Ulmer, Stuttgart.

Wißkirchen, R., Haeupler, H. (1998): Standardliste der Farn- und Blütenpflanzen Deutschlands. - Haeupler, H. [Hrsg.]: Die Farn- und Blütenpflanzen Deutschlands 1: 765 S., Ulmer, Stuttgart.

Zamfir, M., Dai, X., Maarel, E. van der (1999): Bryophytes, lichens and phanerogams in an alvar grassland: relationships at different scales and contributions to plant community pattern. - Ecography 22: 40-52.

Zobel, M. (1987): The classification of estonian Alvars and their plant communities. - Laasimer, L., Kull, T.[Hrsg.]: The Plant Cover of the Estonian SSR - Flora, Vegetation and Ecology: 28-45, Valgus, Tallinn.

Zobel, M. (1992): Plant species coexistence - the role of historical, evolutionary and ecological factors. - Oikos 65: $314-320$. 


\section{Tabellenverzeichnis}

Tab. 1: Mittlere Monats- und Jahrestemperaturen $\left[{ }^{\circ} \mathrm{C}\right]$ an unterschiedlichen Messpunkten (RAUDSEPP \& JAAGUS 2002: 66, vereinfacht).

Tab. 2: Mittlere Monats- und Jahresniederschlagsmengen [mm], sowie die Niederschlagsmengen während der Sommermonate Mai bis August, der Hauptvegetationsperiode April bis Oktober und außerhalb der Vegetationsperiode November bis März an unterschiedlichen Messpunkten (RAUDSEPP \& JAAGUS 2002: 67).

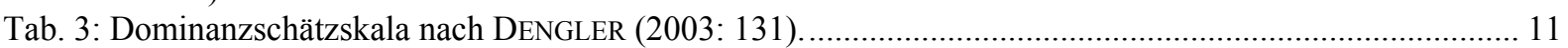

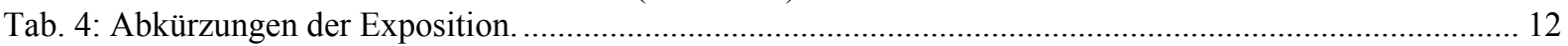

Tab. 5: Schätzskala für den Gesteinsanteil des Oberbodens................................................................ 12

Tab. 6: Skala der Kennwerte für die ermittelte Bodentiefgründigkeit. ..................................................... 12

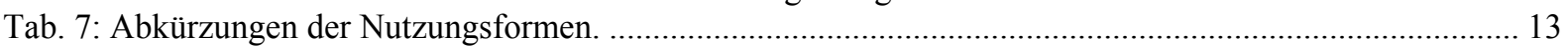

Tab. 8: Skala der Kennwerte für die ermittelte Größe der Trockenrasengebiete........................................... 14

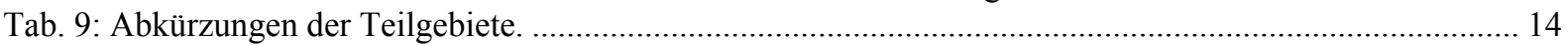

Tab. 10: Carbonatgehalt (leicht modifiziert nach SCHLICHTING et. al. 1995: 45 f.)..................................... 18

Tab. 11: Abkürzungen der syntaxonomischen Hierarchieebenen................................................................. 20

Tab. 12: Mittlere Gesamt- und Gruppenartenzahlen sowie die Deckungsgrade des Caricetum arenariae und seiner Untereinheiten $(n=$ Anzahl der Aufnahmen, $\varnothing=$ arithmetisches Mittel, Min. $=$ Minimum, Max. $=$ Maximum, $\mathrm{s}=$ Standardabweichung)

Tab. 13: Mittlere Gesamt- und Gruppenartenzahlen sowie die Deckungsgrade des Helichryso-Jasionetum und seiner Untereinheiten $(n=$ Anzahl der Aufnahmen, $\varnothing=$ arithmetisches Mittel, Min. $=$ Minimum, Max. $=$ Maximum, $\mathrm{s}=$ Standardabweichung) .

Tab. 14: Mittlere Gesamt- und Gruppenartenzahlen sowie die Deckungsgrade des Festucetum polesicae und seiner Untereinheiten ( $n=$ Anzahl der Aufnahmen, $\varnothing=$ arithmetisches Mittel, Min. $=$ Minimum, Max. $=$ Maximum, $\mathrm{s}=$ Standardabweichung)

Tab. 15: Mittlere Gesamt- und Gruppenartenzahlen sowie die Deckungsgrade des Sileno-Festucetum und seiner Untereinheiten $(n=$ Anzahl der Aufnahmen, $\varnothing=$ arithmetisches Mittel, Min. $=$ Minimum, Max. = Maximum, $\mathrm{s}=$ Standardabweichung).

Tab. 16: Mittlere Gesamt- und Gruppenartenzahlen sowie die Deckungsgrade des Cladonio-Sedetum und seiner Untereinheiten $(n=$ Anzahl der Aufnahmen, $\varnothing=$ arithmetisches Mittel, Min. $=$ Minimum, Max. $=$ Maximum, $\mathrm{s}=$ Standardabweichung).

Tab. 17: Mittlere Gesamt- und Gruppenartenzahlen sowie die Deckungsgrade des Crepido-Allietum und seiner Untereinheiten $(n=$ Anzahl der Aufnahmen, $\varnothing=$ arithmetisches Mittel, Min. $=$ Minimum, Max. $=$ Maximum, $\mathrm{s}=$ Standardabweichung).

Tab. 18: Mittlere Gesamt- und Gruppenartenzahlen sowie die Deckungsgrade der Helictotrichon-Gesellschaft ( $n=$ Anzahl der Aufnahmen, $\varnothing=$ arithmetisches Mittel, Min. $=$ Minimum, Max. $=$ Maximum, $\mathrm{s}=$ Standardabweichung).

Tab. 19: Korrelationsmatrix (Spearman-Rangkorrelation) der Gesamt- und Gruppenartenzahlen sowie der erfassten abiotischen Umweltparameter $(n=167)$. Hochsignifikante Zusammenhänge $(p<0,01)$ sind fett dunkelgrau, auf dem Niveau $p<0,05$ signifikante Zusammenhänge hellgrau unterlegt. .......................... 35

Tab. 20: Übersicht der untersuchten Bodenparameter bezogen auf sämtliche Aufnahmen $(n=$ Anzahl der Aufnahmen, $\varnothing=$ arithmetisches Mittel, $\mathrm{s}=$ Standardabweichung) ..................................................... 36

Tab. 21: Übersicht der untersuchten Bodenparameter getrennt nach den vorkommenden Trockenrasentypen und deren Untereinheiten (vgl. Abschnitt 4.1) ( $n=$ Anzahl der Aufnahmen, Med = Median, $\varnothing=$ Arithmetisches Mittel, $\mathrm{s}=$ Standardabweichung).

Tab. 22: Ergebnisse der Post-hoc-Mehrfachvergleiche (Tukey-HSD) wichtiger Bodenparameter der Trockenrasen auf Saaremaa getrennt nach Hauptgruppen. Übereinstimmende Buchstaben kennzeichnen jeweils homogene Gruppen bezüglich des jeweiligen Parameters auf Basis eines 95 \%Konfidenzintervalles ( $n=$ Anzahl der Aufnahmen).

Tab. 23: Ergebnisse der Post-hoc-Mehrfachvergleiche (Tukey-HSD) wichtiger Bodenparameter in den unterschiedlichen Trockenrasentypen auf Saaremaa. Übereinstimmende Buchstaben kennzeichnen jeweils homogene Gruppen bezüglich des jeweiligen Parameters auf Basis eines 95 \%-Konfidenzintervalles ( $n=$ Anzahl der Aufnahmen).

Tab. 24: Übersicht der prozentualen Deckungsgrade der Vegetation sowie wichtiger Strukturparameter bezogen auf sämtliche Aufnahmen $(n=231)(\varnothing=$ arithmetisches Mittel, $s=$ Standardabweichung).................... 39

Tab. 25: Übersicht der mittleren prozentualen Deckungsgrade der Vegetation sowie wichtiger Strukturparameter getrennt nach den vorkommenden Trockenrasengesellschaften und ihren Untereinheiten $(n=$ Anzahl der Aufnahmen, $\varnothing=$ arithmetisches Mittel, $\mathrm{s}=$ Standardabweichung).

Tab. 26: Ergebnisse der Post-hoc-Mehrfachvergleiche (Tukey-HSD) für die Deckungsgrade der Vegetation und für wichtige Strukturparameter in den unterschiedlichen Trockenrasen auf Saaremaa getrennt nach Hauptgruppen. Übereinstimmende Buchstaben kennzeichnen jeweils homogene Gruppen bezüglich der 
Vegetationsdeckung sowie der Strukturparameter auf Basis eines $95 \%$-Konfidenzintervalles $(n=$ Anzahl der Aufnahmen).

Tab. 27: Ergebnisse der Post-hoc-Mehrfachvergleiche (Tukey-HSD) für die Deckungsgrade der Vegetation und für wichtige Strukturparameter in den unterschiedlichen Trockenrasentypen auf Saaremaa.

Übereinstimmende Buchstaben kennzeichnen jeweils homogene Gruppen bezüglich der

Vegetationsdeckung sowie der Strukturparameter auf Basis eines 95 \%-Konfidenzintervalles $(n=$ Anzahl der Aufnahmen).

Tab. 28: Übersicht der Gesamt- und Gruppenartendichten auf $4 \mathrm{~m}^{2}$, bezogen auf sämtliche Aufnahmen ( $n=$ Anzahl der Aufnahmen, $\varnothing=$ arithmetisches Mittel, $\mathrm{s}=$ Standardabweichung)............................... 43

Tab. 29: Ergebnisse der Post-hoc-Mehrfachvergleiche (Tukey-HSD) für die Gesamt- und Gruppenartendichten auf $4 \mathrm{~m}^{2}$ in den unterschiedlichen Hauptgruppen der Trockenrasen auf Saaremaa. Übereinstimmende Buchstaben kennzeichnen jeweils homogene Gruppen von Hauptgruppen bezüglich der Artendichte auf Basis eines $95 \%$-Konfidenzintervalles $(n=$ Anzahl $)$......

Tab. 30: Ergebnisse der Post-hoc-Mehrfachvergleiche (Tukey-HSD) für die Gesamt- und Gruppenartendichten auf $4 \mathrm{~m}^{2}$ in den unterschiedlichen Trockenrasentypen auf Saaremaa. Übereinstimmende Buchstaben kennzeichnen jeweils homogene Gruppen von Gesellschaften bezüglich der Artendichte auf Basis eines $95 \%$-Konfidenzintervalles $(n=$ Anzahl).

Tab. 31: Übersicht der Gesamt- und Gruppenartendichten auf $4 \mathrm{~m}^{2}$ bezogen auf sämtliche Aufnahmen ( $n=$ Anzahl der Aufnahmen, $\varnothing=$ arithmetisches Mittel, $\mathrm{s}=$ Standardabweichung).....

Tab. 32: Arithmetische Mittel (Ø), Standardabweichungen (s) und Ergebnisse der Post-hoc-Mehrfachvergleiche (Tukey-HSD) für die prozentualen Anteile der Artengruppen am Gesamtartenreichtum auf $4 \mathrm{~m}^{2}$ in den unterschiedlichen Hauptgruppen der Trockenrasen auf Saaremaa. Übereinstimmende Buchstaben kennzeichnen jeweils homogene Gruppen bezüglich der Anteile der Artengruppen am Gesamtartenreichtum auf Basis eines 95 \%-Konfidenzintervalles ( $n=$ Anzahl der Aufnahmen).....

Tab. 33: Arithmetische Mittel (Ø), Standardabweichungen (s) und Ergebnisse der Post-hoc-Mehrfachvergleiche (Tukey-HSD) für die prozentualen Anteile der Artengruppen am Gesamtartenreichtum auf $4 \mathrm{~m}^{2}$ in den unterschiedlichen Trockenrasentypen sowie den Hauptgruppen auf Saaremaa. Übereinstimmende Buchstaben kennzeichnen jeweils homogene Gruppen bezüglich der Anteile der Artengruppen am Gesamtartenreichtum auf Basis eines $95 \%$-Konfidenzintervalles ( $n=$ Anzahl der Aufnahmen).......

Tab. 34: Ergebnisse der schrittweisen multiplen linearen Regressionsanalyse mit der Gesamtartendichte als abhängige Variable und den erfassten abiotischen Parametern als unabhängige Variablen $(n=167)$. Die Variablen wurden in Abhängigkeit des Signifikanzniveaus ihres F-Wertes eingeschlossen $(p<0,05)$ oder entfernt $(p>0,1)$

Tab. 35: Ergebnisse der schrittweisen multiplen linearen Regressionsanalyse mit der Gefäßpflanzenartendichte als abhängige Variable und den erfassten abiotischen Parametern als unabhängige Variablen $(n=167)$. Die Variablen wurden in Abhängigkeit des Signifikanzniveaus ihres F-Wertes eingeschlossen $(p<0,05)$ oder entfernt $(p>0,1)$.

Tab. 36: Ergebnisse der schrittweisen multiplen linearen Regressionsanalyse mit der Moosartendichte als abhängige Variable und den erfassten abiotischen Parametern als unabhängige Variablen $(n=167)$. Die Variablen wurden in Abhängigkeit des Signifikanzniveaus ihres F-Wertes eingeschlossen $(p<0,05)$ oder entfernt $(p>0,1)$.

Tab. 37: Ergebnisse der schrittweisen multiplen linearen Regressionsanalyse mit der Flechtenartendichte als abhängige Variable und den erfassten abiotischen Parametern als unabhängige Variablen $(n=167)$. Die Variablen wurden in Abhängigkeit des Signifikanzniveaus ihres F-Wertes eingeschlossen $(p<0,05)$ oder entfernt $(p>0,1)$.

Tab. 38: Ergebnisse der einfachen Regressionsstatistiken zur Kurvenanpassung mit der Gesamt-, Gefäßpflanzen, Moos- sowie Flechtenartendichte als abhängige Variable und den erfassten abiotischen Parametern als unabhängige Variablen $(n=167)$. Auf dem Niveau $p<0,05$ signifikante Zusammenhänge sind fett gesetzt (Berechnung der Werte: Bodentiefgründigkeit, Größe der Trockenrasengebiete, Gesteinsanteil vgl. Abschnitt 3.1.2; Carbonatgehalt vgl. Abschnitt 3.3.2; $p=$ Signifikanzniveau,

Regressionskoeffizienten: $b_{0}=$ Konstante, $b_{1}=$ linearer Term, $b_{2}=$ quadratischer Term).

Tab. 39: Bivariate Korrelationsanalysen (Pearson) zwischen den Residuen der schrittweisen multiplen linearen Regressionsanalysen und den erfassten Strukturparametern $(n=167)$. Hochsignifikante Zusammenhänge $(p<0,01)$ sind fett gedruckt und dunkelgrau, auf dem Niveau $p<0,05$ signifikante Zusammenhänge hellgrau unterlegt.

Tab. 40: Syntaxonomische Übersicht der im Untersuchungsgebiet nachgewiesenen Trockenrasengesellschaften. Zentralsyntaxa sind mit einem Sternchen (*) gekennzeichnet. 


\section{Abbildungsverzeichnis}

Abb. 1: Mittlere Gesamt- und Gruppenartenzahlen auf $4 \mathrm{~m}^{2}$ sowie deren Standardabweichung in den unterschiedlichen Trockenrasengesellschaften auf Saaremaa.

Abb. 2: Durchschnittliche Anteile der Artengruppen am Gesamtartenreichtum auf $4 \mathrm{~m}^{2}$ in den unterschiedlichen Trockenrasengesellschaften auf Saaremaa. .

\section{Kartenverzeichnis}

Karte 1: Die Insel Saaremaa mit den im Text erwähnten Orten (durchgehende Kreislinie) und Gebieten (gestrichelte Kreislinie) sowie dem angedeuteten Verlauf der Endmoräne (,West-Saaremaa-Hochland“). Kuressaare (1), Halbinsel Pammana (2), Sääre (3), Halbinsel Sörve (4), Insel Vilsandi (5), Raugi (Muhu) (6), Uue-Lõve (7), Karja (8), Muhu (9), Eeriksaare (10), Kaugatoma pank (11), Lõu pank (12), Salme (13), Kihelkonna (14), Naturschutzgebiet Viidumäe (15), Orrissaare (16), Halbinsel Papissaare (17), Läätsa (18), Halbinsel Tagamoisa (19), Tagaranna pank (20), Panga pank (20), Poka (21), Valjala (22), Halbinsel Kübassaare (23), Katri pank (24), Pidula laht (25), Metsa (26), Murika (27), Uudepanga laht (28), Haagi lõugas (29), Kehila (30), Kõõru (31), Pidula laht (32), Mändjala (33),

Tehumardi (34), Kärla (35), Varese Sadam (36), IIpla (37), Atla (38), Nasva (39)............................... 3

Karte 2: Mittlere Windgeschwindigkeiten Estlands (RATHMANN 2003: 33, leicht verändert)............................ 5

Karte 3: Die natürliche Vegetation Saaremaas (aus BoHN et al. 2004); D18 = Alvar-Fichtenmischwälder, D19 = Baltisch-nordwestsarmatische Laub-Fichtenmischwälder, D49 = Osteuropäische psammophytische Kiefernwälder, F70 = Linden-Eichen-Mischwälder, P21 = Nordostbaltische Salvegetation, S9 = Baltische Sphagnum magellanicum-Hochmoorkomplexe, S24 = Kalkreiche Braunmoore.....

Karte 4: Verteilung der untersuchten Trockenrasenflächen über das Untersuchungsgebiet 10

Karte 5: Gliederung der Insel in Teilgebiete. Die Vegetationsaufnahmen sind mit dem Kürzel des jeweiligen

Teilgebietes versehen in welchem sie erstellt wurden.

\section{Anhänge:}

Anhang 1: Gesamtartenliste der in den Vegetationsaufnahmen vorkommenden Gefäßpflanzen, Moose, Flechten und ,Makroalgen“.

Anhang 2: GPS-Daten der Vegetationsaufnahmen.

\section{Beilagenverzeichnis}

Tabelle A: Gekürzte Gesamtstetigkeitstabelle der Trockenrasen Saaremaas.

Tabelle B: Vegetationsaufnahmen des Caricetum arenariae einschließlich Untereinheiten.

Tabelle C: Vegetationsaufnahmen des Helichryso arenarii-Jasionetum litoralis einschließlich Untereinheiten.

Tabelle D: Vegetationsaufnahmen des Festucetum polesicae einschließlich Untereinheiten.

Tabelle E: Vegetationsaufnahmen des Sileno otitae-Festucetum brevipilae einschließlich Untereinheiten.

Tabelle F: Vegetationsaufnahmen des Cladonio symphycarpae-Sedetum albi einschließlich Untereinheiten.

Tabelle G: Vegetationsaufnahmen des Crepido pumilae-Allietum alvarensis einschließlich Untereinheiten.

Tabelle H: Vegetationsaufnahmen der Helictotrichon pratense-[Brachypodietalia]-Gesellschaft.

\section{Abkürzungsverzeichnis}

$\begin{array}{llll}\text { Abb. } & \text { Abbildung } & \text { ges. } & \text { gesamt } \\ \text { Aufl. } & \text { Auflage } & \text { Hrsg. } & \text { Herausgeber } \\ b & \text { Regressionskoeffizient } & n & \text { Anzahl } \\ b_{0} & \text { Regressionskonstante } & p & \text { Signifikanzniveau } \\ b_{1} & \text { Regressionskoeffizient des linearen Terms } & r & \text { Korrelationskoeffizient } \\ b_{2} & \text { Regressionskoeffizient des quadratischen Terms } & r^{2} & \text { erklärte Varianz } \\ \text { bed. } & \text { bedeckt } & \text { Tab. } & \text { Tabelle } \\ \text { Begr. } & \text { Begründer } & \text { ü. NN } & \text { über Normalnull }\end{array}$




\section{Danksagung}

Mein Dank gilt:

In besonderem Maße Dr. Jürgen Dengler für die erstklassige Betreuung, sein Engagement und die Geduld während meiner gesamten Diplomarbeitsphase, sowie der Ausbildung in meiner Studienzeit. Er war stets für Fragen offen, konnte mir bei allen Problemen helfen, versorgte mich mit der richtigen Literatur und stellte mir seine noch unveröffentlichten Daten zur Verfügung. Außerdem bestätigte er mir die nachbestimmten FestucaArten sowie Tortula calcicolens.

Arne Hein (Lüneburg) für die Übersetzung der schwedischen Texte zur Beschreibung der Sippen Allium schoenoprasum var. alvarense, Crepis tectorum ssp. pumila sowie Festuca oelandica.

Aveliina Helm (Tartu) für die Bereitstellung noch unveröffentlichter Daten, die Unterkunft während der Zeit in Tartu und Informationen zu den Alvaren Saaremaas. Sie war außerdem stets offen für Fragen und konnte mir einige gute Literaturtips geben.

Christian Dolnik (Kiel) für die Einführung in die Dünnschichtchromatographie von Flechtensubstanzen sowie die Bestimmung des Bryum erythrocarpum-Komplexes und dem daraus resultierenden Neunachweis für Estland: Bryum radiculosum.

Dieter Stengel (Lüneburg) für die Plexiglasrähmchen, den „Tiefgründigkeitsmesser“ und das GPSÜbertragungskabel.

Günter Gottschlich (Tübingen) für die Bestimmung der kritischen Habichtskräuter aus der Untergattung Pilosella.

Hartmut Meier (Lüneburg) für die Bereitstellung der GPS-Software sowie des Präzisions-Planimeters.

Kaadri Kullapere (Vilsandi) für die Betretungserlaubnis im Nationalpark Vilsandi und den Internetzugang.

Laurens Sparrius (Gouda) für zwei lehrreiche „Bestimmungsabende“, an denen er mir einen umfassenden Einblick in Estlands Flechtenflora vermittelte und mir Artmerkmale und Schwierigkeiten bei der Determination kritischer Sippen verdeutlichte. Außerdem machte er mich auf die für Estland neue Art Agonimia globulifera aufmerksam, ließ mir die geeignete Bestimmungsliteratur für diese Flechte zukommen und bestätigte oder revidierte mir einige unklare Arten.

Mare Leis (Tartu) für einen ganzen Tag „Moosbestimmungskurs“.

Meelis Pärtel (Tartu) für Sonderdrucke, die Bereitstellung noch unveröffentlichter Daten und die Führung durch das biologische Institut der Universität Tartu.

Sascha Schandrin (Izhevsk) für die Übersetzung der russischen Klimakarten.

Swantje Löbel (Uppsala) für die Bereitstellung noch unveröffentlichter Daten.

Tiina Randlaane (Tartu) für eine kurze Gesteinsflechteneinführung.

Universitätsgesellschaft Lüneburg e.V. für die finanzielle Unterstützung meiner Estlandreise.

Allen, die mir hilfreiche Ratschläge geben konnten.

Meiner Familie (Renate, Wilfried und Bon) ganz besonders für die finanzielle und mentale Unterstützung während meines Studiums und der Diplomarbeit.

Meiner Freundin Anne Busch für ihre Unterstützung und die Hilfe während meiner gesamten Diplomarbeit. 


\section{Anhang 1: Verzeichnis der Pflanzensippen}

\section{Gesamtartenliste - Gefäßpflanzen:}

Achillea millefolium ssp. millefolium

Acinos arvensis

Campanula rotundifolia

Agrimonia eupatoria

Capsella bursa-pastoris

Agrostis capillaris

Agrostis stolonifera

Agrostis vinealis

Alchemilla glaucescens

Allium oleraceum

Cardamine hirsuta

Carex arenaria

Carex caryophyllea

Carex ericetorum

Carex flacca

Carex hirta

Allium schoenoprasum var. alvarense

Carex muricata

Allium schoenoprasum

Allium vineale

Alyssum montanum ssp. gmelinii

Ammophila arenaria

Anagallis arvensis

Androsace septentrionalis

Antennaria dioica

Anthemis tinctoria

Anthoxanthum odoratum

Anthyllis vulneraria ssp. vulneraria

Aphanes arvensis

Aquilegia vulgaris

Arabidopsis thaliana

Arabis hirsuta

Arenaria serpyllifolia ssp. glutinosa

Arenaria serpyllifolia ssp. serpyllifolia

Carex ornithopoda ssp. ornithopoda

Carex pilulifera

Carex spicata

Carlina vulgaris

Centaurea jacea

Centaurea scabiosa ssp. scabiosa

Cerastium arvense ssp. arvense

Cerastium holosteoides

Cerastium semidecandrum

Chaenorhinum minus

Chenopodium album

Cirsium acaule

Convallaria majalis

Convolvulus arvensis

Cornus sanguinea ssp. sanguinea

Corylus avellana

Arrhenatherum elatius

Crepis tectorum („outcrop-form“)

Asperula tinctoria

Crepis tectorum ssp. tectorum

Astragalus danicus

Barbarea vulgaris ssp. vulgaris

Dactylis glomerata ssp. glomerata

Danthonia decumbens ssp. decumbens

Berberis vulgaris

Daucus carota

Berteroa incana

Botrychium lunaria

Deschampsia flexuosa

Dianthus arenarius

Brachypodium pinnatum

Dianthus deltoides

Briza media

Echium vulgare

Bromus erectus

Elymus repens ssp. repens

Bromus hordeaceus ssp. hordeaceus

Epilobium angustifolium

Calamagrostis epigejos

Epipactis atrorubens

Campanula glomerata

Equisetum arvense

Campanula persicifolia

Equisetum cf. pratense 
Equisetum variegatum

Erigeron acris ssp. acris

Erophila verna

Eryngium maritimum

Erysium hieraciifolium

Euphrasia spec.

Euphrasia stricta

Festuca arundinacea

Festuca cf. oelandica

Festuca nigescens

Festuca ovina

Festuca polesica

Festuca rubra ssp. arenaria

Festuca rubra ssp. rubra

Filipendula ulmaria

Filipendula vulgaris

Fragaria vesca

Fragaria viridis

Galium album ssp. album

Galium boreale

Galium cf. saxatile

Galium verum

Geranium molle

Geranium pusillum

Geranium robertianum ssp. robertianum

Geranium sanguineum

Gnaphalium sylvaticum

Gymnadenia conopsea

Gypsophila fastigiata

Helianthemum nummularium ssp. nummularium

Helictotrichon pratense

Helictotrichon pubescens ssp. pubescens

Hepatica nobilis

Heracleum sphondylium ssp. sibiricum

Herniaria glabra

Hieracium caesium

Hieracium cf. bifidum

Hieracium cf. brachiatum

Hieracium cf. iseranum

Hieracium lactucella

Hieracium peletieranum
Hieracium pilosella

Hieracium piloselloides

Hieracium subgen. Hieracium

Hieracium subgen. Pilosella

Hieracium umbellatum

Hieracium zizianum

Honckenya peploides

Hornungia petraea

Hypericum perforatum

Hypochaeris maculata

Inula salicina

Jasione montana

Juniperus communis ssp. communis

Knautia arvensis

Koeleria glauca

Lapsana communis

Lathyrus pratensis

Leontodon autumnalis

Leontodon hispidus

Leucanthemum vulgare

Leymus arenarius

Linaria vulgaris

Linum catharticum

Lolium multiflorum

Lotus corniculatus ssp. corniculatus

Luzula campestris

Luzula pilosa

Matricaria discoidea

Medicago falcata

Medicago lupulina

Medicago $\times$ varia

Melampyrum arvense

Melampyrum pratense

Melica nutans

Melilotus albus

Mycelis muralis

Myosotis arvensis ssp. arvensis

Myosotis ramosissima

Myosotis stricta

Myosurus minimus

Oenothera rubricaulis 
Ononis arvensis

Ononis spinosa ssp. spinosa

Origanum vulgare ssp. vulgare

Phleum bertolonii

Phleum phleoides

Pimpinella nigra

Pimpinella saxifraga

Pinus sylvestris ssp. sylvestris

Plantago lanceolata

Plantago major ssp. major

Plantago maritima

Plantago media

Platanthera bifolia

Poa angustifolia

Poa annua

Poa compressa

Poa pratensis

Polygala amarella

Polygala comosa

Potentilla argentea agg.

Potentilla incana

Potentilla reptans

Potentilla tabernaemontani

Potentilla $\times$ subarenaria

Primula veris

Prunella vulgaris

Pseudolysimachion spicatum

Pulsatilla pratensis ssp. pratensis

Ranunculus auricomus agg.

Ranunculus bulbosus

Ranunculus nemorosus

Ranunculus polyanthemoides

Ranunculus polyanthemos

Rhamnus cathartica

Rhinanthus minor

Ribes alpinum

Rosa sherardii

Rosa spec.

Rubus caesius

Rumex acetosa

Rumex acetosa agg.
Rumex acetosella

Rumex cf. crispus

Sagina nodosa

Saxifraga granulata

Saxifraga tridactylites

Scabiosa columbaria

Scleranthus annuus

Scleranthus perennis

Scorzonera humilis

Sedum acre

Sedum album

Senecio jacobaea ssp. jacobaea

Seseli libanotis

Sesleria caerulea

Silene latifolia ssp. alba

Silene nutans

Silene viscaria

Silene vulgaris ssp. vulgaris

Solidago virgaurea

Sonchus oleraceus

Sorbus intermedia

Stellaria graminea

Taraxacum sect. Obliqua

Taraxacum sect. Ruderalia

Tetragonolobus maritimus

Thymus serpyllum

Tragopogon pratensis

Trifolium arvense

Trifolium campestre

Trifolium dubium

Trifolium medium

Trifolium montanum

Trifolium pratense

Trifolium repens

Tripleurospermum maritimum

Tripleurospermum perforatum

Tussilago farfara

Valerianella locusta

Veronica arvensis

Veronica chamaedrys ssp. chamaedrys

Veronica officinalis 
Veronica verna

Vicia cracca

Vicia hirsuta

Vicia lathyroides

Vicia sepium

Vicia tetrasperma

Vincetoxicum hirundinaria

Viola arvensis ssp. arvensis

Gesamtartenliste - Moose:

Amblystegium serpens

Barbula convoluta

Barbula unguiculata

Brachythecium albicans

Brachythecium rutabulum

Brachythecium velutinum

Bryum algovicum

Bryum argenteum

Bryum caespiticium

Bryum capillare

Bryum pallescens

Bryum radiculosum

Calliergonella cuspidata

Campylium calcareum

Campylium chrysophyllum

Campylium polygamum

Campylium sommerfeltii

Cephaloziella cf. hampeana

Cephaloziella divaricata

Ceratodon conicus

Ceratodon purpureus

Cirriphyllum piliferum

Climacium dendroides

Ctenidium molluscum

Dicranum scoparium

Distichium inclinatum

Ditrichum flexicaule

Encalypta rhaptocarpa

Encalypta streptocarpa

Eurhynchium praelongum

Fissidens dubius

Funaria hygrometrica
Viola canina

Viola hirta

Viola pumila

Viola rupestris

Viola tricolor ssp. tricolor

Grimmia pulvinata

Hedwigia ciliata

Homalothecium lutescens

Hylocomium splendens

Hypnum cupressiforme var. cupressiforme

Hypnum cupressiforme var. lacunosum

Lophozia excisa

Myurella julacea

Orthotrichum spec.

Phascum cuspidatum

Plagiomnium affine

Plagiomnium cuspidatum

Pleurozium schreberi

Polytrichum juniperinum

Polytrichum piliferum

Preissia quadrata

Ptilidium ciliare

Racomitrium canescens

Rhodobryum roseum

Rhytidiadelphus squarrosus

Riccia bifurca

Riccia ciliata

Riccia sorocarpa

Scapania calcicola

Schistidium apocarpum agg.

Scleropodium purum

Thuidium abietinum

Thuidium philibertii

Tortella fragilis

Tortella inclinata

Tortella tortuosa

Tortula calcicolens 
Tortula muralis

Weissia brachycarpa

Tortula ruraliformis

Tortula ruralis

\section{Gesamtartenliste - Flechten:}

Acarospora glaucocarpa

Cladonia crispata var. crispata

Acarospora smaragdula

Cladonia deformis

Agonimia globulifera

Cladonia fimbriata

Agonimia tristicula

Cladonia floerkeana

Amandinea punctata

Cladonia foliacea

Anaptychia ciliaris

Cladonia furcata

Aspicilia calcarea

Cladonia glauca

Aspicilia cinerea

Cladonia gracilis

Aspicilia contorta ssp. contorta

Cladonia novochlorophaea

Aspicilia contorta ssp. hoffnanniana

Cladonia phyllophora

Aspicilia grisea

Cladonia pleurota

Bacidia arceutina

Cladonia pocillum

Bacidia bagliettoana

Cladonia polycarpoides

Caloplaca cerina

Cladonia pyxidata agg.

Caloplaca cerinelloides

Cladonia rangiferina

Caloplaca citrina

Cladonia rangiformis

Caloplaca crenulatella

Cladonia rei

Caloplaca holocarpa

Cladonia subrangiformis

Caloplaca lithophila

Cladonia subulata

Caloplaca sinapisperma

Cladonia sulphurina

Candelariella aurella

Candelariella vitellina

Cladonia symphycarpia

Catillaria chalybeia

Catillaria lenticularis

Cetraria aculeata

Cetraria ericetorum

Cetraria islandica

Cetraria muricata

Cetraria sepincola

Cladonia arbuscula

Cladonia cariosa

Cladonia caespiticia

Cladonia ciliata

Cladonia coniocraea

Cladonia convoluta

Cladonia cornuta

Cladonia uncialis ssp. biuncialis

Cladonia verticillata

Collema bachmanianum

Collema crispum

Collema tenax

Diploschistes muscorum

Diplotomma alboatrum

Evernia prunastri

Flavocetraria nivalis

Fulgensia bracteata

Gyalecta jenensis

Hypogymnia physodes

Lecanora albescens

Lecanora carpinea

Lecanora dispersa 
Lecanora expallens

Lecanora intricata

Lecanora polytropa

Lecanora pulicaris

Lecanora symmicta

Lecidea fuscoatra

Lecidea plana

Lecidella carpathica

Lecidella cf. euphorea

Lecidella elaeochroma

Lecidella stigmatea

Lepraria incana

Leptogium gelatinosum

Mycobilimbia berengeriana

Myxobilimbia sabuletorum

Ochrolechia microstictoides

Parmelia sulcata

Peltigera canina

Peltigera didactyla

Peltigera hymenina

Peltigera neckeri

Peltigera polydactylon

Peltigera rufescens

Phaeophysica orbicularis

Physcia caesia

\section{Gesamtartenliste - „Makroalgen“:}

Nostoc spec.

Trentepohlia spec.
Physcia tenella

Placynthiella icmalea

Placynthiella oligotropha

Protoblastenia rupestris

Protoparmeliopsis muralis

Pseudevernia furfuracea

Psora decipiens

Ramalina farinacea

Ramalina fastigiata

Rinodina gennarii

Stereocaulon incrustatum

Toninia sedifolia

Trapeliopsis granulosa

Usnea spec.

Verrucaria bryoctona

Verrucaria muralis

Verrucaria nigrescens

Verrucaria tectorum

Vulpicidia tubulosus

Xanthoparmelia conspersa

Xanthoria candelaria

Xanthoria parietina

Xanthoria polycarpa 


\section{Anhang 2: GPS-Daten der Vegetationsaufnahmen (UTM WGS-84)}

Aufnahmenummer/ Rechtswert/ Hochwert

\begin{tabular}{|c|c|c|c|c|c|}
\hline E01 & 552.328 & 6.466 .352 & L06 & 558.340 & 6.467 .037 \\
\hline E02 & 552.346 & 6.466 .510 & L07 & 557.844 & 6.465 .480 \\
\hline E03 & 552.242 & 6.466 .362 & L08 & 556.439 & 6.465 .731 \\
\hline E04 & 553.203 & 6.463 .929 & L09 & 556.696 & 6.467 .096 \\
\hline E05 & 553.197 & 6.463 .922 & L10 & 556.627 & 6.466 .333 \\
\hline J01 & 573.776 & 6.448 .846 & L11 & 559.905 & 6.464 .748 \\
\hline J02 & 573.785 & 6.448 .852 & L12 & 559.978 & 6.464 .518 \\
\hline J03 & 573.790 & 6.448 .846 & L13 & 563.232 & 6.463 .222 \\
\hline J04 & 573.782 & 6.448 .843 & L14 & 563.787 & 6.462 .833 \\
\hline J05 & 573.781 & 6.448 .848 & L15 & 554.316 & 6.462 .376 \\
\hline J06 & 574.346 & 6.450 .102 & L16 & 554.790 & 6.462 .593 \\
\hline J07 & 574.377 & 6.450 .135 & N01 & 581.045 & 6.454 .708 \\
\hline J08 & 576.959 & 6.452 .947 & N02 & 581.061 & 6.454 .667 \\
\hline J09 & 579.054 & 6.453 .859 & N03 & 584.200 & 6.455 .832 \\
\hline J10 & 579.325 & 6.453 .914 & N04 & 584.204 & 6.455 .826 \\
\hline J11 & 579.037 & 6.454 .035 & N05 & 584.205 & 6.455 .835 \\
\hline J12 & 577.331 & 6.452 .841 & N06 & 584.196 & 6.455 .837 \\
\hline J13 & 576.986 & 6.452 .638 & N07 & 584.195 & 6.455 .827 \\
\hline J14 & 576.925 & 6.452 .752 & Ö01 & 611.902 & 6.494 .685 \\
\hline J15 & 576.908 & 6.452 .746 & Ö02 & 599.707 & 6.495 .787 \\
\hline J16 & 576.175 & 6.451 .970 & Ö03 & 587.334 & 6.495 .563 \\
\hline J17 & 574.046 & 6.450 .687 & Ö04 & 587.286 & 6.495 .578 \\
\hline J18 & 574.457 & 6.450 .278 & Ö05 & 587.383 & 6.495 .623 \\
\hline J19 & 574.489 & 6.450 .363 & P01 & 557.846 & 6.469 .523 \\
\hline J20 & 574.551 & 6.450 .460 & P02 & 558.201 & 6.469 .394 \\
\hline J21 & 574.557 & 6.450 .453 & P03 & 558.017 & 6.469 .421 \\
\hline J22 & 574.561 & 6.450 .459 & P04 & 557.801 & 6.469 .254 \\
\hline J23 & 574.557 & 6.450 .466 & P05 & 557.840 & 6.469 .217 \\
\hline J24 & 574.556 & 6.450 .459 & S01 & 573.479 & 6.446 .358 \\
\hline K01 & 570.342 & 6.443 .183 & S02 & 572.314 & 6.444 .370 \\
\hline K02 & 570.346 & 6.443 .162 & S03 & 572.311 & 6.444 .187 \\
\hline K03 & 570.298 & 6.442 .985 & S04 & 573.101 & 6.446 .260 \\
\hline K04 & 570.296 & 6.442 .947 & S05 & 573.246 & 6.445 .701 \\
\hline K05 & 570.447 & 6.443 .179 & T01 & 558.005 & 6.476 .116 \\
\hline K06 & 570.261 & 6.442 .654 & T02 & 556.807 & 6.481 .530 \\
\hline K07 & 569.779 & 6.441 .615 & T03 & 555.132 & 6.482 .967 \\
\hline L01 & 557.219 & 6.463 .611 & T04 & 557.650 & 6.474 .498 \\
\hline L02 & 555.152 & 6.462 .326 & T05 & 560.860 & 6.476 .168 \\
\hline L03 & 560.404 & 6.469 .269 & T06 & 561.044 & 6.473 .932 \\
\hline L04 & 560.019 & 6.468 .149 & T07 & 557.658 & 6.474 .512 \\
\hline L05 & 560.318 & 6.469 .352 & T08 & 553.702 & 6.484 .263 \\
\hline
\end{tabular}




\begin{tabular}{|c|c|c|c|c|c|}
\hline T09 & 553.127 & 6.481 .889 & X15 & 553.920 & 6.456 .919 \\
\hline T10 & 553.262 & 6.481 .973 & X16 & 556.775 & 6.455 .495 \\
\hline T11 & 553.297 & 6.481 .946 & $\mathbf{X} 17$ & 556.806 & 6.455 .814 \\
\hline T12 & 552.601 & 6.482 .311 & X18 & 572.215 & 6.471 .316 \\
\hline T13 & 557.866 & 6.475 .764 & X19 & 568.759 & 6.474 .719 \\
\hline T14 & 557.220 & 6.478 .537 & $\mathbf{X} 20$ & 568.735 & 6.474 .732 \\
\hline T15 & 552.719 & 6.481 .451 & X21 & 564.724 & 6.475 .630 \\
\hline T16 & 552.623 & 6.481 .638 & $\mathrm{X} 22$ & 564.699 & 6.475 .666 \\
\hline T17 & 552.295 & 6.481 .913 & $\mathrm{X} 23$ & 565.021 & 6.479 .538 \\
\hline T18 & 551.824 & 6.482 .253 & X24 & 565.023 & 6.479 .560 \\
\hline T19 & 551.984 & 6.482 .151 & $\mathrm{X} 25$ & 565.027 & 6.479 .580 \\
\hline T20 & 553.327 & 6.481 .868 & X26 & 573.697 & 6.462 .927 \\
\hline T21 & 553.264 & 6.481 .823 & $\mathbf{X} 27$ & 575.425 & 6.461 .808 \\
\hline T22 & 553.268 & 6.481 .817 & X28 & 582.304 & 6.461 .618 \\
\hline T23 & 553.269 & 6.481 .823 & X29 & 582.506 & 6.461 .606 \\
\hline T24 & 553.260 & 6.481 .825 & $\mathbf{X 3 0}$ & 573.686 & 6.466 .609 \\
\hline T25 & 553.261 & 6.481 .814 & X31 & 572.770 & 6.465 .681 \\
\hline T26 & 552.957 & 6.481 .549 & X32 & 568.841 & 6.474 .767 \\
\hline T27 & 558.094 & 6.476 .048 & X33 & 568.840 & 6.474 .772 \\
\hline T28 & 558.089 & 6.476 .043 & X34 & 568.838 & 6.474 .765 \\
\hline T29 & 558.099 & 6.476 .045 & X35 & 568.854 & 6.474 .770 \\
\hline T30 & 558.095 & 6.476 .055 & X36 & 568.842 & 6.474 .769 \\
\hline T31 & 558.089 & 6.476 .055 & X37 & 568.904 & 6.474 .759 \\
\hline V01 & 549.293 & 6.471 .336 & X38 & 568.877 & 6.474 .768 \\
\hline V02 & 547.450 & 6.471 .632 & X39 & 570.087 & 6.473 .204 \\
\hline V03 & 547.732 & 6.471 .409 & X40 & 570.078 & 6.473 .205 \\
\hline V04 & 548.600 & 6.470 .303 & X41 & 570.077 & 6.473 .195 \\
\hline V05 & 548.804 & 6.470 .519 & $\mathrm{X} 42$ & 570.087 & 6.473 .194 \\
\hline V06 & 548.910 & 6.470 .573 & X43 & 570.082 & 6.473 .200 \\
\hline V07 & 548.884 & 6.471 .101 & X44 & 572.815 & 6.468 .900 \\
\hline X01 & 573.663 & 6.466 .592 & X45 & 572.427 & 6.469 .252 \\
\hline X02 & 573.353 & 6.466 .189 & $\mathrm{X} 46$ & 576.417 & 6.465 .338 \\
\hline X03 & 573.267 & 6.466 .162 & X47 & 576.418 & 6.465 .346 \\
\hline X04 & 576.190 & 6.465 .446 & X48 & 576.412 & 6.465 .339 \\
\hline X05 & 578.194 & 6.463 .990 & X49 & 576.420 & 6.465 .334 \\
\hline X06 & 578.179 & 6.463 .943 & X50 & 576.425 & 6.465 .340 \\
\hline X07 & 572.785 & 6.465 .706 & X51 & 563.894 & 6.475 .496 \\
\hline X08 & 572.777 & 6.465 .715 & X52 & 563.898 & 6.475 .489 \\
\hline X09 & 567.308 & 6.467 .224 & X53 & 563.897 & 6.475 .499 \\
\hline X10 & 568.526 & 6.460 .672 & X54 & 563.889 & 6.475 .497 \\
\hline X11 & 566.252 & 6.461 .135 & X55 & 563.890 & 6.475 .488 \\
\hline $\mathrm{X} 12$ & 559.778 & 6.459 .001 & X56 & 565.575 & 6.475 .928 \\
\hline X13 & 553.928 & 6.456 .870 & X57 & 577.482 & 6.465 .170 \\
\hline X14 & 553.923 & 6.456 .863 & X58 & 577.489 & 6.465 .173 \\
\hline
\end{tabular}




\begin{tabular}{|c|c|c|c|c|c|}
\hline X59 & 577.480 & 6.465 .176 & Z05 & 612.575 & 6.467 .813 \\
\hline X60 & 577.477 & 6.465 .166 & Z06 & 608.983 & 6.473 .179 \\
\hline X61 & 577.486 & 6.465 .164 & Z07 & 606.110 & 6.476 .198 \\
\hline X62 & 577.871 & 6.463 .993 & Z08 & 608.017 & 6.481 .201 \\
\hline X63 & 577.876 & 6.463 .987 & Z09 & 606.285 & 6.473 .857 \\
\hline X64 & 577.874 & 6.463 .996 & Z10 & 607.534 & 6.480 .890 \\
\hline X65 & 577.865 & 6.463 .994 & Z11 & 607.541 & 6.480 .888 \\
\hline X66 & 577.866 & 6.463 .987 & $\mathbf{Z 1 2}$ & 607.535 & 6.480 .893 \\
\hline X67 & 577.741 & 6.464 .803 & Z13 & 607.527 & 6.480 .891 \\
\hline X68 & 577.879 & 6.463 .972 & Z14 & 607.532 & 6.480 .883 \\
\hline X69 & 577.948 & 6.463 .822 & Z15 & 618.850 & 6.483 .112 \\
\hline X70 & 581.934 & 6.461 .534 & Z16 & 628.494 & 6.485 .464 \\
\hline X71 & 581.936 & 6.461 .527 & Z17 & 633.547 & 6.483 .306 \\
\hline X72 & 556.838 & 6.455 .864 & Z18 & 634.441 & 6.480 .470 \\
\hline X73 & 556.836 & 6.455 .858 & Z19 & 629.062 & 6.484 .440 \\
\hline X74 & 556.844 & 6.455 .859 & Z20 & 596.097 & 6.464 .422 \\
\hline X75 & 556.842 & 6.455 .868 & Z21 & 596.237 & 6.463 .467 \\
\hline X76 & 556.832 & 6.455 .868 & $\mathbf{Z 2 2}$ & 596.094 & 6.463 .304 \\
\hline $\mathbf{X} 77$ & 555.323 & 6.456 .765 & & & \\
\hline X78 & 553.994 & 6.457 .198 & & & \\
\hline X79 & 553.989 & 6.457 .197 & & & \\
\hline X80 & 553.998 & 6.457 .192 & & & \\
\hline X81 & 554.001 & 6.457 .201 & & & \\
\hline X82 & 553.992 & 6.457 .205 & & & \\
\hline X83 & 553.832 & 6.456 .906 & & & \\
\hline X84 & 557.865 & 6.458 .518 & & & \\
\hline Y01 & 571.536 & 6.488 .823 & & & \\
\hline Y02 & 570.552 & 6.490 .189 & & & \\
\hline Y03 & 570.474 & 6.490 .214 & & & \\
\hline Y04 & 570.411 & 6.490 .202 & & & \\
\hline Y05 & 570.406 & 6.490 .208 & & & \\
\hline Y06 & 570.407 & 6.490 .198 & & & \\
\hline Y07 & 570.418 & 6.490 .199 & & & \\
\hline Y08 & 570.416 & 6.490 .208 & & & \\
\hline Y09 & 570.291 & 6.490 .216 & & & \\
\hline Y10 & 577.770 & 6.487 .483 & & & \\
\hline Y11 & 574.879 & 6.492 .877 & & & \\
\hline Y12 & 574.866 & 6.492 .865 & & & \\
\hline Y13 & 583.850 & 6.493 .778 & & & \\
\hline Y14 & 581.420 & 6.493 .442 & & & \\
\hline Z01 & 612.570 & 6.467 .809 & & & \\
\hline Z02 & 612.566 & 6.467 .811 & & & \\
\hline Z03 & 612.569 & 6.467 .802 & & & \\
\hline Z04 & 612.576 & 6.467 .807 & & & \\
\hline
\end{tabular}


Tab. A: Gekürzte Gesamtstetigkeitstabelle der Trockenrasen Saaremaas. Die Stetigkeiten für Syntaxa oberhalb der Assoziation sind als arithmetisches Mittel der Stetigkeiten in den zugehörigen Assoziationen berechnet. Für jede Sippe ist ein gerundeter prozentualer Stetigkeitswert angegeben. Eine „0“ gibt an, dass der Wert unter 0,5 \% liegt, ein Punkt hingegen, dass die Art in den entsprechenden Aufnahmen nicht vorkommt. Dunkelgrau unterlegte Stetigkeitswerte kennzeichnen Charakterarten (C), hellgraue stehen für eine Klassendifferenzialart bzw. die obere Ebene einer transgressiven Charakterart. Einfach umrahmte Werte kennzeichnen eine Differenzialart (D). Charakter- bzw. Differenzialarten des jeweiligen Syntaxons können als regional gültig angesehen werden, auch wenn diese Einteilung teilweise von der überregionalen Klassifikation abweicht. Zentralsyntaxa sind mit einem Sternchen ${ }^{*}$ ) gekennzeichnet. Eine Spalte wird kursiv abgebildet, wenn das jeweilige Syntaxon durch weniger als zehn Vegetationsaufnahmen repräsentiert ist und somit nur eine eingeschränkte Beurteilung der Charakter- und Differenzialarten möglich ist. Zu den „Sonstigen“ wurden Arten mit einer Gesamtstetigkeit von mindestens $15 \%$ gestellt. Territoriale Charakterarten sind ab einer prozentualen Stetigkeit von $2 \%$, Differenzialarten ab $5 \%$ dargestellt.

\begin{tabular}{|c|c|c|c|c|c|c|c|c|c|c|c|c|c|}
\hline \multirow[b]{4}{*}{ Syntaxon } & \multirow{4}{*}{ 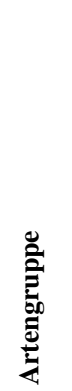 } & \multirow{4}{*}{ 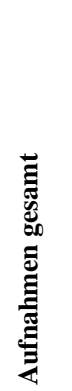 } & \multirow{4}{*}{ 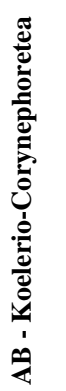 } & \multirow{4}{*}{ 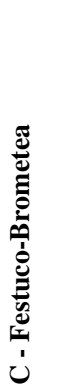 } & \multirow{4}{*}{ 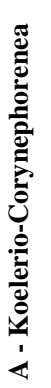 } & \multirow{4}{*}{ 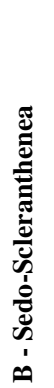 } & \multicolumn{6}{|c|}{ AB - Koelerio-Corynephoretea } & \multirow{2}{*}{$\begin{array}{c}\text { C - Festuco- } \\
\text { Brometea }\end{array}$} \\
\hline & & & & & & & \multicolumn{4}{|c|}{ A - Koelerio-Corynephorenea } & \multicolumn{2}{|c|}{$\begin{array}{c}\begin{array}{c}\text { B - Sedo- } \\
\text { Scleranthenea }\end{array} \\
\text { B.a }\end{array}$} & \\
\hline & & & & & & & A.a & \multicolumn{2}{|c|}{ A.b } & \multirow{2}{*}{$\begin{array}{l}\text { A.c } \\
\text { A4 }\end{array}$} & B.a1a & B.a1b & \multirow{2}{*}{$\begin{array}{l}\text { C.a } \\
\text { C1 }\end{array}$} \\
\hline & & & & & & & A1 & A2 & A3 & & B1 & B2 & \\
\hline Anzahl der Assoziationen & & 7 & 6 & 1 & 4 & 2 & & & & & & & \\
\hline Anzahl der Aufnahmen & & 231 & 164 & 67 & 85 & 79 & 6 & 12 & 43 & 24 & 25 & 54 & 67 \\
\hline Mittlere Gesamtartenzahl & & 32 & 28 & 41 & 23 & 40 & 15 & 22 & 23 & 30 & 32 & 48 & 41 \\
\hline Mittlere Artenzahl Gefäßpflanzen & & 16 & 13 & 30 & 10 & 20 & 2 & 8 & 10 & 21 & 18 & 21 & 30 \\
\hline Mittlere Artenzahl Moose & & 7 & 6 & 8 & 4 & 11 & 2 & 4 & 6 & 5 & 8 & 14 & 8 \\
\hline Mittlere Artenzahl Flechten & & 8 & 8 & 2 & 8 & 8 & 11 & 10 & 8 & 4 & 5 & 11 & 2 \\
\hline
\end{tabular}

A, B und C - Koelerio-Corynephoretea mit Festuco-Brometea

D Galium verum

Ditrichum flexicaule

Artemisia campestris

Thymus serpyllum

Thuidium abietinum

Hypnum cupressiforme var. lacunosum

Tortella inclinata

$\begin{array}{llll}\text { G } & 66 & 52 & 81 \\ \text { M } & 59 & 45 & 54 \\ \text { G } & 50 & 51 & 36 \\ \text { G } & 53 & 45 & 48 \\ \text { M } & 45 & 35 & 48 \\ \text { M } & 41 & 29 & 48 \\ \text { M } & 42 & 35 & 21\end{array}$

$\begin{array}{ll}49 & 57 \\ 24 & 87 \\ 50 & 55 \\ 53 & 30 \\ 26 & 52 \\ 21 & 46 \\ 22 & 61\end{array}$

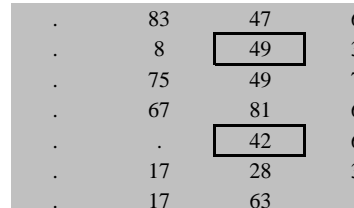

$\begin{array}{cccc}67 & 32 & 81 & 81 \\ 38 & 84 & 89 & 54 \\ 75 & 52 & 57 & 36 \\ 63 & 4 & 56 & 48 \\ 63 & 56 & 48 & 48 \\ 38 & 32 & 59 & 48 \\ 8 & 48 & 74 & 21\end{array}$

AB - Koelerio-Corynephoretea

C Ceratodon purpureus

Sedum acre

Peltigera rufescens

Cladonia pocillum

Cerastium semidecandrum

D Brachythecium albicans

Arenaria serpyllifolia ssp. glutinosa

$\begin{array}{cc}\text { M } & 47 \\ \text { G } & 51 \\ \text { F } & 38 \\ \text { F } & 30 \\ \text { G } & 21 \\ \text { M } & 31 \\ \text { G } & 25\end{array}$
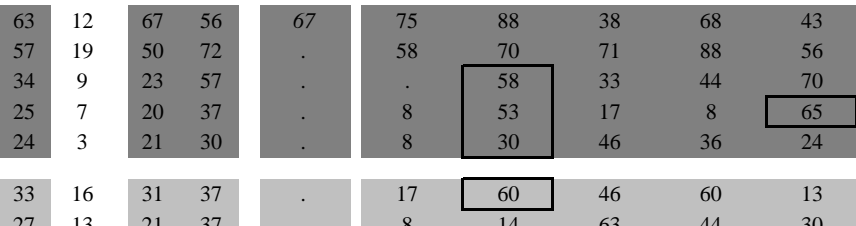

A - Koelerio-Corynephorenea

C Racomitrium canescens

Tortula ruraliformis

Cetraria islandica

Pulsatilla pratensis ssp. pratensis

D Cetraria aculeata

Carex arenaria

Cladonia furcata

Cephaloziella divaricata

Hieracium umbellatum

Cladonia arbuscula

Cladonia phyllophora

Placynthiella oligotropha

Cladonia glauca

Cladonia subulata

Hypogymnia physodes
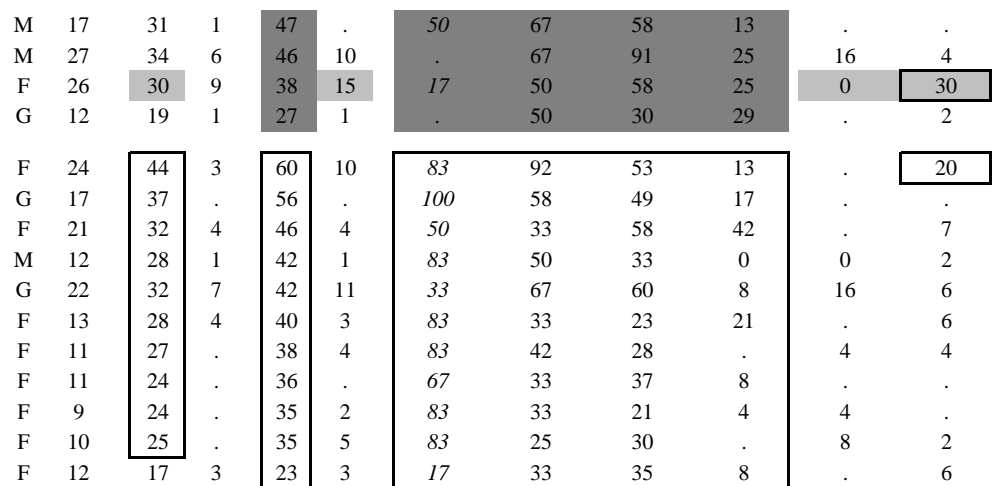

\begin{tabular}{|cccc|}
\hline 83 & 92 & 53 & 13 \\
100 & 58 & 49 & 17 \\
50 & 33 & 58 & 42 \\
83 & 50 & 33 & 0 \\
33 & 67 & 60 & 8 \\
83 & 33 & 23 & 21 \\
83 & 42 & 28 &. \\
67 & 33 & 37 & 8 \\
83 & 33 & 21 & 4 \\
83 & 25 & 30 &. \\
17 & 33 & 35 & 8 \\
\hline
\end{tabular}

A.a - Corynephoretalia canescentis *

D Cladonia fimbriata

Cladonia gracilis

$\begin{array}{llllll}\text { F } & 6 & 13 & 3 & 17 & 6\end{array}$

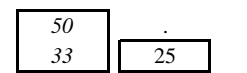

2

A2 - Helichryso arenarii-Jasionetum litoralis *

C Cladonia foliacea

Androsace septentrionalis

D Plantago maritima

Honckenya peploides 
A3 - Festucetum polesicae

Festuca polesica

Koeleria glauca

Dianthus arenarius

Alyssum montanum ssp. gmelinii

Cetraria ericetorum

$\begin{array}{ccccc}\text { G } & 17 & 16 & . & 23 \\ \text { G } & 10 & 9 & . & 14 \\ \text { G } & 8 & 7 & . & 11 \\ \text { G } & 5 & 4 & . & 7 \\ \text { F } & 5 & 5 & . & 6\end{array}$

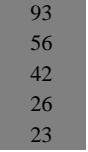

A.c - Trifolio arvensis-Festucetalia ovinae

C Peltigera canina

Myosotis stricta

$\begin{array}{cccccc}\text { F } & 7 & 9 & 1 & 8 & 10 \\ \text { G } & 2 & 2 & 3 & 3 & \text {. } \\ & & & & & \\ \text { M } & 10 & 11 & 9 & 12 & 7 \\ \text { G } & 23 & 13 & 48 & 11 & 19 \\ \text { G } & 5 & 6 & 4 & 8 & 2 \\ \text { G } & 8 & 9 & 6 & 8 & 12 \\ \text { M } & 9 & 7 & 15 & 7 & 6\end{array}$

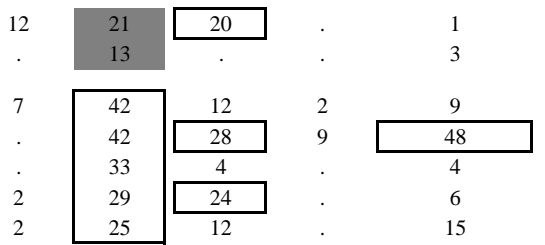

A4 - Sileno-Festucetum *

C Trifolium arvense

Phleum phleoides

Polytrichum juniperinum

Dianthus deltoides

$\begin{array}{cccccc}\text { G } & 13 & 16 & 9 & 18 & 11 \\ \mathrm{G} & 6 & 6 & 6 & 6 & 6 \\ \mathrm{M} & 3 & 5 & 1 & 7 & . \\ \mathrm{G} & 3 & 2 & 4 & 3 & . \\ \mathrm{G} & 2 & 2 & 3 & 3 & .\end{array}$

B - Sedo-Scleranthenea (bzw. B.a Alysso alyssoidis-Sedetalia)

$\begin{array}{cccccc}\text { M } & 31 & 18 & 30 & 2 & 49 \\ \text { F } & 19 & 15 & 10 & 7 & 30 \\ \text { G } & 3 & 3 & . & . & 9 \\ \text { G } & 28 & 23 & 15 & 6 & 56 \\ \text { M } & 25 & 22 & 13 & 9 & 49 \\ \text { F } & 17 & 13 & 12 & 4 & 30\end{array}$

Cladonia subrangiformis

Hornungia petraed

Acinos arvensis

Bryum caespiticium

Bacidia bagliettoana

\begin{tabular}{lllll|l} 
G & 43 & 29 & 45 & 15 & 57
\end{tabular}

Festuca ovina

Poa compressa

Barbula convoluta

Nostoc spec.

$\begin{array}{lllllll}G & 34 & 23 & 40 & 7 & 54\end{array}$

Bryum argenteum

Sagina nodosa

Echium vulgare

Potentilla argentea agg.

Bromus hordeaceus ssp. hordeaceus

Arenaria serpyllifolia ssp. serpyllifolia

1a - Tortello tortuosae-Sedenion albi *

D Erophila verna

Tortula ruralis

Daucus carota

\begin{tabular}{cccccc|}
$\mathrm{G}$ & 9 & 10 & 3 & 4 & 22 \\
$\mathrm{M}$ & 5 & 6 & 1 & 1 & 16 \\
$\mathrm{G}$ & 6 & 4 & 10 & 1 & 10
\end{tabular}

- Cladonio symphycarpae-Sedetum albi

C Saxifraga tridactylites

G $3 \quad 5 \quad . \quad 15$

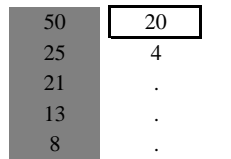

9
6
1
4
3

B.a1b - Tortello rigentis-Helianthemenion oelandici

C Barbula unguiculata

Encalypta streptocarpa

Encalypta rhaptocarpa

$\begin{array}{cccccc}\text { M } & 16 & 10 & 10 & 2 & 27 \\ M & 14 & 10 & 6 & . & 31 \\ M & 5 & 3 & & & 10\end{array}$

D Agrostis stolonifera

Weissia brachycarpa

Cladonia rangiformis

Bryum capillare

Collema tenax
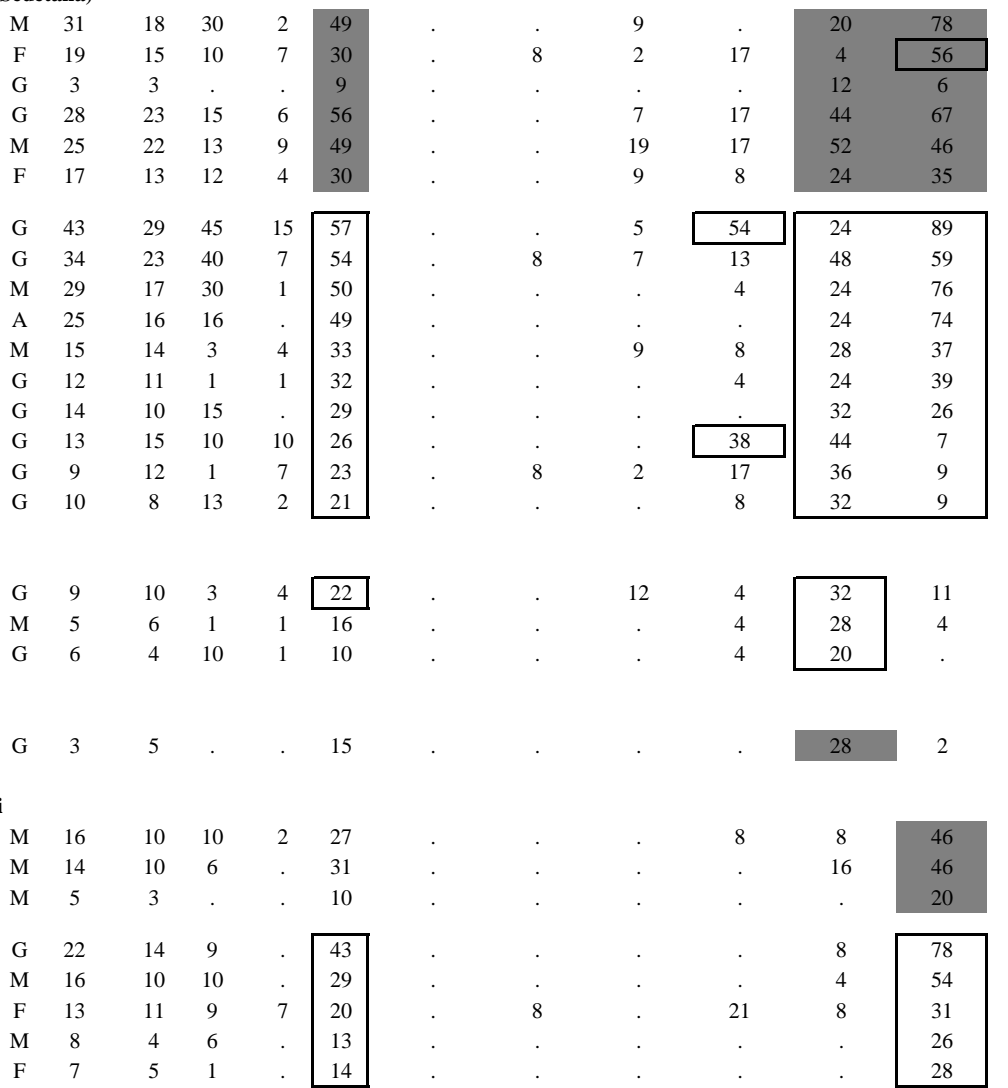

30
10

\begin{tabular}{|c|c|c|c|c|}
\hline & 9 & . & 20 & 78 \\
\hline 8 & 2 & 17 & 4 & 56 \\
\hline . & . & . & 12 & 6 \\
\hline . & 7 & 17 & 44 & 67 \\
\hline & 19 & 17 & 52 & 46 \\
\hline & 9 & 8 & 24 & 35 \\
\hline . & 5 & 54 & 24 & 89 \\
\hline 8 & 7 & 13 & 48 & 59 \\
\hline . & . & 4 & 24 & 76 \\
\hline . & . & . & 24 & 74 \\
\hline . & 9 & 8 & 28 & 37 \\
\hline . & . & 4 & 24 & 39 \\
\hline . & . & . & 32 & 26 \\
\hline . & . & 38 & 44 & 7 \\
\hline 8 & 2 & 17 & 36 & 9 \\
\hline & . & 8 & 32 & 9 \\
\hline
\end{tabular}

B2 - Crepido pumilae-Allietum alvarensis

C Sedum album

Cladonia symphycarpia

Artemisia rupestris

Distichium inclinatum

Riccia bifurca

Fulgensia bracteata

Toninia sedifolia

Tortella fragilis

Psora decipiens

Agonimia globulifera

Euphrasia stricta

Campylium sommerfeltii

Myurella julacea

Agonimia tristicula

Allium schoenoprasum var. alvarense

Leptogium gelatinosum

Crepis tectorum ("outcrop-Form")

$\begin{array}{cccccc}\text { G } & 19 & 14 & 3 & . & 43 \\ \text { F } & 22 & 16 & 7 & 5 & 38 \\ \text { G } & 16 & 11 & 6 & . & 32 \\ \text { M } & 15 & 11 & 6 & . & 32 \\ \text { M } & 11 & 8 & 4 & . & 23 \\ \text { F } & 9 & 7 & . & . & 20 \\ F & 9 & 6 & . & . & 19 \\ \text { M } & 9 & 6 & 3 & . & 18 \\ \text { F } & 8 & 6 & 1 & . & 17 \\ \text { F } & 8 & 5 & 1 & . & 16 \\ \text { G } & 9 & 7 & 1 & 2 & 17 \\ \text { M } & 9 & 5 & 6 & . & 16 \\ \text { M } & 6 & 5 & . & . & 14 \\ F & 6 & 4 & 1 & . & 12 \\ \text { G } & 5 & 3 & . & . & 10 \\ \text { F } & 5 & 4 & 1 & . & 12 \\ \text { G } & 4 & 3 & . & . & 9\end{array}$


C - Festuco-Bometea (bzw. C.a - Brachypodietalia pinnati)

\begin{tabular}{|c|c|c|}
\hline \multirow{18}{*}{ 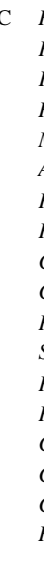 } & Helictotrichon pratense & G \\
\hline & Pimpinella nigra & G \\
\hline & Filipendula vulgaris & G \\
\hline & Homalothecium lutescens & M \\
\hline & Medicago lupulina & G \\
\hline & Anthyllis vulneraria ssp. vulneraria & G \\
\hline & Potentilla tabernaemontani & G \\
\hline & Pseudolysimachion spicatum & G \\
\hline & Campylium chrysophyllum & M \\
\hline & Cirsium acaule & G \\
\hline & Potentilla $\times$ subarenaria & G \\
\hline & Senecio jacobaea ssp. jacobaea & G \\
\hline & Pimpinella saxifraga & G \\
\hline & Potentilla incana & G \\
\hline & Carex caryophyllea & G \\
\hline & Carlina vulgaris & G \\
\hline & Carex ericetorum & G \\
\hline & Helictotrichon pubescens ssp. pubescens & G \\
\hline \multirow{21}{*}{ D } & Briza media & G \\
\hline & Hieracium pilosella & G \\
\hline & Dactylis glomerata ssp. glomerata & G \\
\hline & Sesleria caerulea & G \\
\hline & Brachythecium rutabulum & G \\
\hline & Fragaria viridis & G \\
\hline & Knautia arvensis & G \\
\hline & Carex flacca & G \\
\hline & Lotus corniculatus ssp. corniculatus & G \\
\hline & Fissidens dubius & M \\
\hline & Solidago virgaurea & G \\
\hline & Campanula rotundifolia & G \\
\hline & Ctenidium molluscum & M \\
\hline & Galium album ssp. album & G \\
\hline & Trifolium pratense & G \\
\hline & Luzula campestris & G \\
\hline & Phleum bertolonii & G \\
\hline & Polygala amarella & G \\
\hline & Plagiomnium affine & M \\
\hline & Vicia cracca & G \\
\hline & Taraxacum sect. Ruderalia & G \\
\hline
\end{tabular}
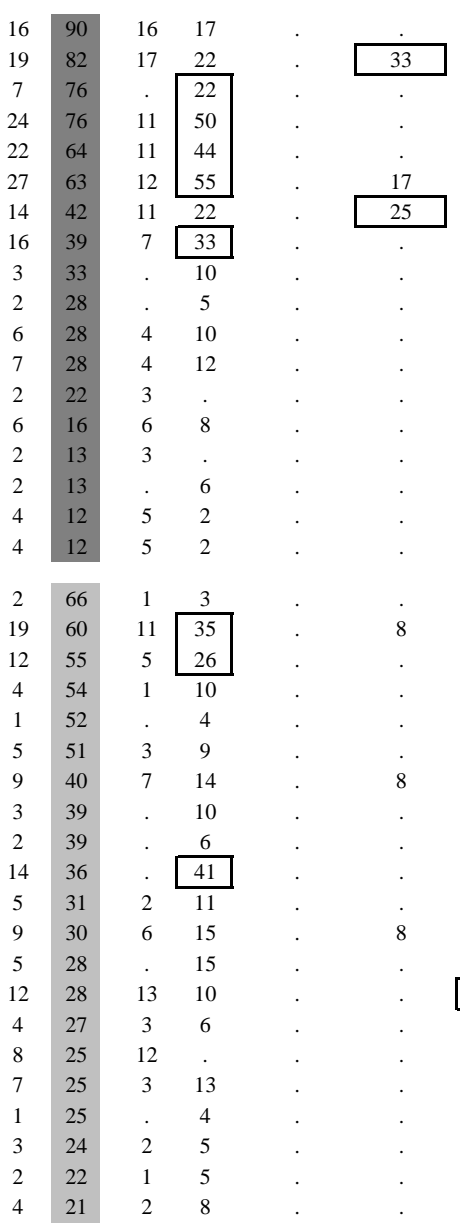

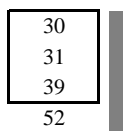

39

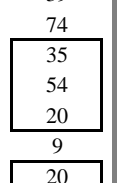

20

11

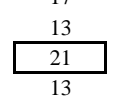

.
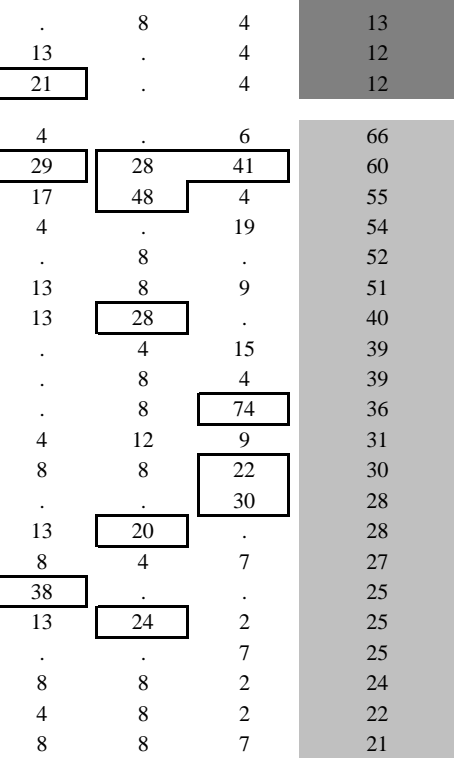

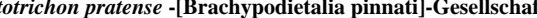

Ranunculus bulbosus

Plantago media

Trifolium montanum

Thuidium philibertii

Helianthemum nummularium ssp. num.

Bromus erectus

Ononis spinosa ssp. spinosa

Campanula glomerate

Leontodon hispidus

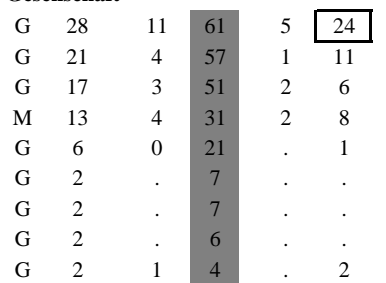

Sonstige Arten ( $\geq 15 \%$ Stetigkeit)

Sonstige (Gefäßpflanzen)

Achillea millefolium ssp. millefolium

Plantago lanceolata

Festuca rubra ssp. rubra

Centaurea jacea

Linum catharticum

Hypericum perforatum

Galium boreale

Leucanthemum vulgare

Carex pilulifera

Asperula tinctoria

Pinus sylvestris ssp. sylvestris (juv.)

Juniperus communis ssp. com. (juv.)

$\begin{array}{cccccc}\text { G } & 47 & 29 & 76 & 19 & 50 \\ \text { G } & 42 & 25 & 76 & 17 & 40 \\ \text { G } & 34 & 17 & 70 & 10 & 30 \\ \text { G } & 32 & 12 & 64 & 1 & 33 \\ \text { G } & 31 & 10 & 63 & 2 & 27 \\ \text { G } & 29 & 17 & 48 & 8 & 36 \\ \text { G } & 25 & 10 & 48 & 2 & 27 \\ \text { G } & 25 & 13 & 46 & 3 & 33 \\ \text { G } & 19 & 8 & 39 & 7 & 10 \\ \text { G } & 17 & 7 & 30 & 3 & 15 \\ \text { G } & 16 & 8 & 25 & 6 & 10 \\ \text { G } & 15 & 6 & 25 & 1 & 17\end{array}$

Sonstige (epigäische Moose)

Bryum spec.

Dicranum scoparium

$\begin{array}{llllll}M & 16 & 12 & 9 & 5 & 28\end{array}$

M $\quad 14 \quad 15 \quad 10 \quad 14 \quad 15$

Sonstige (epigäische Flechten)

Cladonia pyxidata agg.

Sonstige (epilithische Flechten)

Verrucaria nigrescens

Verrucaria muralis

Protoblastenia rupestris
$\begin{array}{lllllllllllll}\mathrm{F} & 28 & 36 & 16 & 47 & 14 & 33 & 58 & 58 & 38 & 8 & 19 & 16\end{array}$

$\begin{array}{llllll}\text { F } & 39 & 33 & 30 & 12 & 75\end{array}$

$\begin{array}{llllll}\mathrm{F} & 38 & 29 & 33 & 11 & 67\end{array}$ 
Tab. B: Vegetationsaufnahmen des Caricetum arenariae einschließlich Untereinheiten. Für die aufgeführten Sippen ist zusätzlich ein Kürzel der taxonomischen Artengruppe $(\mathrm{G}=$

Gefäßpflanzen, $\mathrm{M}=$ Moose, $\mathrm{F}=$ Flechten) sowie deren prozentuale Stetigkeiten in den

Vegetationsaufnahmen des Caricetum arenariae angegeben. Eingerahmte Felder kennzeichnen einen gegen die andere Untereinheit differenzierenden Artenblock $(C, D=$ territoriale Charakterbzw. Differenzialart des jeweiligen Syntaxons; $d=$ Differenzialart unterhalb des

Assoziationsniveaus; $*$ = Zentralsyntaxon). Am Ende der Tabelle sind außerdem die Kopfdaten aufgelistet.

\begin{tabular}{|c|c|c|c|c|c|c|c|c|}
\hline \multirow[b]{3}{*}{ Aufnahmenummer } & \multirow{3}{*}{ 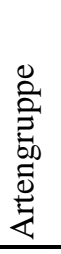 } & \multirow{3}{*}{ 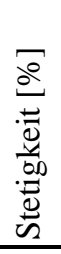 } & \multicolumn{6}{|c|}{ A1 - Caricetum arenariae } \\
\hline & & & \multirow{2}{*}{$\frac{\text { A1a }}{\text { Ö05 }}$} & \multicolumn{5}{|c|}{ A1b } \\
\hline & & & & $\mathrm{T} 21$ & $\mathrm{~T} 22$ & $\mathrm{~T} 20$ & $\mathrm{~T} 23$ & $\mathrm{~T} 10$ \\
\hline Gesamtartenzahl & & & 1 & 24 & 20 & 16 & 18 & 9 \\
\hline Artenzahl Gefäßpflanzen & & & 1 & 2 & 3 & 1 & 1 & 1 \\
\hline Artenzahl Moose & & & 0 & 5 & 2 & 3 & 3 & 1 \\
\hline Artenzahl Flechten & & & 0 & 17 & 15 & 12 & 14 & 7 \\
\hline
\end{tabular}

\section{A1b - Strauchflechtenreiche Ausbildung}

d Cetraria aculeata

Cladonia arbuscula

Cladonia floerkeana

Cladonia glauca

Cladonia phyllophora

Cladonia subulata

Cladonia cornuta

Cladonia deformis

Cladonia furcata

Cladonia pleurota

Cladonia rangiferina

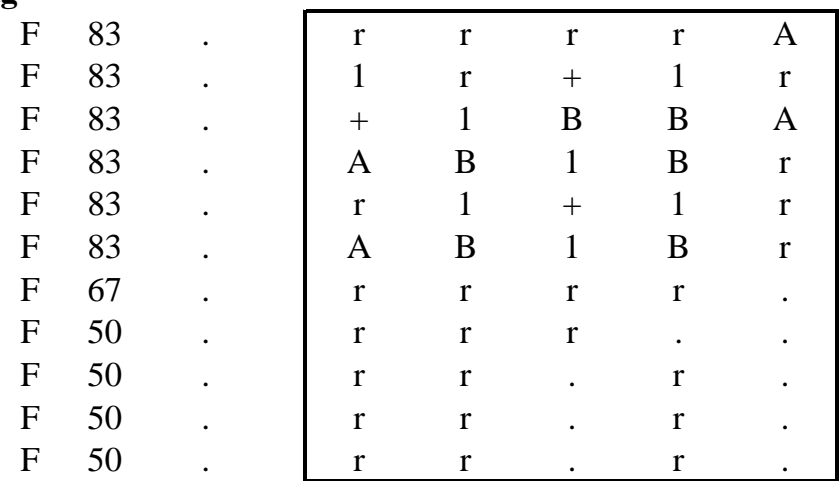

A.a - Corynephoretalia canescentis *

D Cladonia fimbriata

Cladonia gracilis

A - Koelerio-Corynephorenea

C Racomitrium canescens Cetraria islandica

$\begin{array}{cccccccc}\text { M } & 50 & . & 1 & . & \text { A } & \text { r } & \text {. } \\ \text { F } & 17 & . & . & . & \text { r } & . & \text {. } \\ \text { G } & 100 & 1 & 3 & 3 & 3 & 3 & \text { B } \\ \text { M } & 83 & . & \text { B } & \text { A } & \text { B } & \text { B } & \text { B } \\ \text { F } & 67 & . & \text { r } & \text { r } & \text { B } & \text { r } & . \\ \text { G } & 33 & . & \text { r } & \text { r } & . & . & \text {. } \\ \text { F } & 17 & . & . & . & . & . & \text { r }\end{array}$

AB - Koelerio-Corynephoretea

C Ceratodon purpureus

M 67

3

B B

\section{Sonstige (Gefäßpflanzen)}

Leymus arenarius

Sonstige (epigäische Moose)

Dicranum scoparium

Polytrichum piliferum

M 17

M 17

Sonstige (epigäische Flechten)

Cladonia crispata var. crispata

Cladonia pyxidata agg.

Cladonia sulphurina

Cladonia verticillata

Sonstige (epixyle Flechten)

Hypogymnia physodes

Placynthiella oligotropha 
Tab. C: Vegetationsaufnahmen des Helichryso-Jasionetum einschließlich Untereinheiten. Für die aufgeführten Sippen ist zusätzlich das Kürzel der taxonomischen Artengruppe ( $\mathrm{G}=$ Gefäßpflanzen, $\mathrm{M}=$ Moose, $\mathrm{F}=$ Flechten) sowie deren prozentuale Stetigkeiten in den Vegetationsaufnahmen des Helichryso-Jasionetum angegeben. Eingerahmte Felder kennzeichnen einen gegen die andere Untereinheit differenzierenden Artenblock ( $\mathrm{AC}=$ Assoziationscharakterart; $\mathrm{C}, \mathrm{D}=$ territoriale Charakter- bzw. Differenzialart des jeweiligen Syntaxons; $d=$ Differenzialart unterhalb des Assoziationsniveaus; * = Zentralsyntaxon). Am Ende der Tabelle sind außerdem die Kopfdaten aufgelistet.

\begin{tabular}{|c|c|c|c|c|c|c|c|c|c|c|c|c|c|}
\hline \multirow[b]{3}{*}{ Aufnahmenummer } & \multirow{3}{*}{ 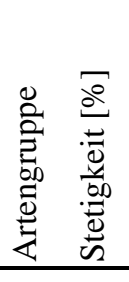 } & \multicolumn{12}{|c|}{ A2 - Helichryso arenarii-Jasionetum litoralis } \\
\hline & & \multicolumn{8}{|c|}{ A2a } & \multicolumn{4}{|c|}{$\mathbf{A} 2 \mathbf{b}$} \\
\hline & & T11 & $\mathrm{T} 25$ & $\mathrm{~T} 24$ & $\mathrm{~T} 15$ & $\mathrm{~T} 16$ & T09 & $\mathrm{T} 12$ & $\mathrm{~T} 18$ & T17 & T19 & T05 & $\mathrm{X} 16$ \\
\hline Gesamtartenzahl & & 7 & 22 & 25 & 19 & 17 & 25 & 31 & 20 & 29 & 26 & 20 & 18 \\
\hline Artenzahl Gefäßpflanzen & & 5 & 4 & 6 & 8 & 8 & 4 & 12 & 11 & 10 & 9 & 16 & 4 \\
\hline Artenzahl Moose & & 0 & 4 & 3 & 5 & 4 & 5 & 7 & 2 & 5 & 4 & 2 & 2 \\
\hline Artenzahl Flechten & & 2 & 14 & 16 & 6 & 5 & 16 & 12 & 7 & 14 & 13 & 2 & 12 \\
\hline
\end{tabular}

A2 - Helichryso arenarii-Jasionetum litoralis *

$\begin{array}{lllllllllllllllllll}\text { C } & \text { Cladonia foliacea } & \text { F } & 58 & . & . & \text { r } & \text { r } & . & + & \text { A } & \text { A } & \text { B } & 1 \\ \text { D } & \text { Pimpinella nigra } & \text { G } & 33 & . & . & . & . & . & . & \text { A } & \text { r } & & \text { r } & \text { r }\end{array}$

\section{A2a - Hieracium umbellatum Ausbildung}

d Hieracium umbellatum

Cladonia pyxidata agg.

Cephaloziella divaricata

Cetraria islandica

Cladonia phyllophora

Cladonia arbuscula

Cladonia cf. rei

Cladonia glauca

Placynthiella oligotropha

Cladonia cornuta

Cladonia deformis

Cladonia floerkeana

Cladonia gracilis

Cladonia rangiferina

Cladonia subulata

Festuca rubra ssp. arenaria

Honckenya peploides

Brachythecium albicans

Dicranum scoparium

Cladonia crispata var. crispata

Cladonia pleurota

\begin{tabular}{|c|c|c|c|c|c|c|c|c|c|}
\hline $\mathrm{G}$ & 67 & + & + & A & $\mathrm{r}$ & + & + & + & $\mathrm{r}$ \\
\hline $\mathrm{F}$ & 58 & . & 1 & $\mathrm{r}$ & 1 & A & $\mathrm{r}$ & 1 & A \\
\hline $\mathrm{M}$ & 50 & . & A & A & 1 & + & B & 1 & \\
\hline $\mathrm{F}$ & 50 & $\mathrm{r}$ & & . & 1 & $\mathrm{r}$ & $\mathrm{r}$ & $\mathrm{r}$ & A \\
\hline $\mathrm{F}$ & 42 & . & $\mathrm{r}$ & + & & . & B & 1 & . \\
\hline $\mathrm{F}$ & 33 & . & A & 3 & & . & A & + & . \\
\hline F & 33 & . & & & A & A & & A & 1 \\
\hline F & 33 & . & B & A & & $\mathrm{r}$ & 1 & . & . \\
\hline$F$ & 33 & . & $\mathrm{r}$ & $\mathrm{r}$ & & . & $\mathrm{r}$ & 1 & . \\
\hline $\mathrm{F}$ & 25 & . & $\mathrm{r}$ & $\mathrm{r}$ & . & . & $\mathrm{r}$ & . & . \\
\hline $\mathrm{F}$ & 25 & . & $\mathrm{r}$ & $\mathrm{r}$ & & . & $\mathrm{r}$ & . & . \\
\hline$F$ & 25 & . & A & + & & . & 1 & . & . \\
\hline $\mathrm{F}$ & 25 & . & $\mathrm{r}$ & . & • & . & $\mathrm{r}$ & + & . \\
\hline $\mathrm{F}$ & 25 & . & + & A & & . & $\mathrm{r}$ & . & . \\
\hline$F$ & 25 & . & A & A & . & . & + & . & . \\
\hline G & 25 & A & . & . & . & & . & $\mathrm{r}$ & $\mathrm{r}$ \\
\hline $\mathrm{G}$ & 25 & 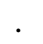 & . & . & A & & & + & + \\
\hline $\mathrm{M}$ & 17 & . & . & . & 1 & & & A & . \\
\hline $\mathrm{M}$ & 17 & . & $\mathrm{r}$ & . & • & . & . & A & . \\
\hline$F$ & 17 & . & $\mathrm{r}$ & $\mathrm{r}$ & . & . & • & . & . \\
\hline $\mathrm{F}$ & 17 & & $\mathrm{r}$ & $\mathrm{r}$ & & & & & \\
\hline
\end{tabular}

A2b - Verrucaria spp.-Potentilla tabernaemontani Ausbildung

$\begin{array}{ccc}\text { d AC Androsace septentrionalis } & \text { G } & 25 \\ \text { Plantago maritima } & \text { G } & 25 \\ \text { Potentilla tabernaemontani } & \text { G } & 25 \\ \text { Diploschistes muscorum } & \text { F } & 17 \\ \text { Stereocaulon incrustatum } & \text { F } & 17 \\ \text { Anthyllis vulneraria ssp. vulneraria } & \text { G } & 17 \\ \text { Tortella inclinata } & \text { M } & 17 \\ \text { epilithische Arten: } & & \\ \text { Verrucaria muralis } & \text { F } & 25 \\ \text { Verrucaria nigrescens } & \text { F } & 25 \\ \text { Lecanora dispersa } & \text { F } & 17 \\ \text { Lecanora intricata } & \text { F } & 17 \\ \text { Lecanora polytropa } & \text { F } & 17 \\ \text { Protoblastenia rupestris } & \text { F } & 17\end{array}$

\section{A - Koelerio-Corynephorenea}

C Racomitrium canescens

Tortula ruraliformis

Pulsatilla pratensis ssp. pratensis

D Cetraria aculeata

Carex arenaria

Cladonia furcata

Hypogymnia physodes

\begin{tabular}{|c|c|c|c|c|c|c|c|c|c|c|c|}
\hline M & 67 & . & $\mathrm{r}$ & $\mathrm{r}$ & 3 & B & 3 & & & 3 & \\
\hline M & 67 & . & . & . & $\mathrm{r}$ & $\mathrm{r}$ & . & 1 & A & t & \\
\hline G & 50 & . & . & . & A & A & ${ }^{\circ}$ & 1 & A & 1 & \\
\hline $\mathrm{F}$ & 92 & 1 & $\mathrm{r}$ & $\mathrm{r}$ & A & A & + & B & B & & \\
\hline G & 58 & A & 3 & 3 & A & 1 & 3 & . & . & . & \\
\hline $\mathrm{F}$ & 33 & . & . & . & . & . & A & A & $r$ & . & \\
\hline $\mathrm{F}$ & 33 & . & . & . & $\mathrm{r}$ & . & . & 1 & + & . & \\
\hline
\end{tabular}




\begin{tabular}{|c|c|c|c|c|c|c|c|c|c|c|c|c|c|c|c|}
\hline & \multirow[b]{3}{*}{ Aufnahmenummer } & \multirow{3}{*}{\multicolumn{2}{|c|}{ 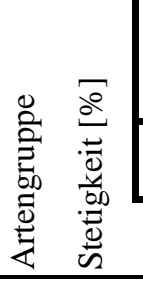 }} & \multicolumn{12}{|c|}{ A2 - Helichryso arenarii-Jasionetum litoralis } \\
\hline & & & & \multicolumn{8}{|c|}{$\mathbf{A 2 a}$} & \multicolumn{4}{|c|}{$\mathbf{A 2 b}$} \\
\hline & & & & T11 & $\mathrm{T} 25$ & $\mathrm{~T} 24$ & T15 & T16 & T09 & $\mathrm{T} 12$ & $\mathrm{~T} 18$ & T17 & $\mathrm{T} 19$ & T05 & $\mathrm{X} 16$ \\
\hline & Gesamtartenzahl & & & 7 & 22 & 25 & 19 & 17 & 25 & 31 & 20 & 29 & 26 & 20 & 18 \\
\hline & Artenzahl Gefäßpflanzen & & & 5 & 4 & 6 & 8 & 8 & 4 & 12 & 11 & 10 & 9 & 16 & 4 \\
\hline & Artenzahl Moose & & & 0 & 4 & 3 & 5 & 4 & 5 & 7 & 2 & 5 & 4 & 2 & 2 \\
\hline & Artenzahl Flechten & & & 2 & 14 & 16 & 6 & 5 & 16 & 12 & 7 & 14 & 13 & 2 & 12 \\
\hline \multicolumn{16}{|c|}{ AB - Koelerio-Corynephoretea } \\
\hline \multirow[t]{2}{*}{$\mathrm{C}$} & Ceratodon purpureus & M & 75 & . & B & B & B & 3 & A & A & B & $\mathrm{r}$ & 1 & . & . \\
\hline & Sedum acre & G & 58 & . & . & . & + & + & . & 1 & 1 & $\mathrm{r}$ & . & A & B \\
\hline \multicolumn{16}{|c|}{ AB und C - Koelerio-Corynephoretea und Festuco-Brometea } \\
\hline \multirow[t]{4}{*}{$\mathrm{D}$} & Galium verum & $\mathrm{G}$ & 83 & $\mathrm{~A}$ & $\mathrm{r}$ & + & A & + & A & $\mathrm{r}$ & 1 & . & . & + & A \\
\hline & Artemisia campestris & G & 75 & $\mathrm{r}$ & . & . & 1 & 1 & . & $\mathrm{r}$ & + & $\mathrm{r}$ & $\mathrm{r}$ & A & $\mathrm{B}$ \\
\hline & Thymus serpyllum & G & 67 & . & . & $\mathrm{r}$ & A & A & A & B & 3 & 1 & A & . & . \\
\hline & Hypnum cupressiforme var. lacunosum & M & 17 & . & . & . & . & . & . & $\mathrm{A}$ & . & . & . & . & $\mathrm{r}$ \\
\hline \multicolumn{16}{|c|}{ Sonstige (Gefäßpflanzen) } \\
\hline & Rumex acetosella & G & 25 & . & . & $\mathrm{r}$ & . & . & . & $\mathrm{r}$ & . & . & . & $\mathrm{r}$ & \\
\hline \multicolumn{16}{|c|}{$\begin{array}{l}\text { Außerdem kommen je einmal vor: } \\
\text { Gefäßpflanzen: Arenaria serpyllifolia } \text { ssp. glutinosa T05: +; Bromus hordeaceus ssp. hordeaceus T05: r; Calamagrostis epigejos T25: r; } \\
\text { Campanula rotundifolia T12: r; Cardamine hirsuta T24: r; Cerastium semidecandrum T05: +; Elymus repens ssp. repens X16: r; Erigeron } \\
\text { acris ssp. acris T19: r; Eryngium maritimum T18: +; Hieracium pilosella T17: r; Jasione montana T05: r; Knautia arvensis T05: 1; Poa } \\
\text { compressa T05: r; Rumex acetosa agg. T05: r; Scleranthus annuus T05: 1; Taraxacum sect. Obliqua T18: r; Taraxacum spec. T17: r; } \\
\text { Trifolium arvense T05: A; Epigäische Moose: Ditrichum flexicaule T09: 1; Polytrichum juniperinum T12: A; Polytrichum piliferum T09: } \\
\text { A; Epilithische Moose: Racomitrium canescens T17: r; Epigäische Flechten: Cladonia pocillum T17: +; Cladonia rangiformis T12: +; } \\
\text { Cladonia subrangiformis X16: r; Cladonia sulphurina T24: r; Cladonia verticillata T24: r; Epilithische Flechten: Acarospora smaragdula } \\
\text { T17: r; Aspicilia cinerea T19: r; Caloplaca citrina X16: r; Caloplaca crenulatella X16: r; Caloplaca holocarpa X16: r; Candelariella aurella } \\
\text { X16: r; Candelariella vitellina T19: r; Lecanora albescens X16: r; Lecidella cf. euphorea T17: r; Lecidella stigmatea T17: +; } \\
\text { Protoparmeliopsis muralis T17: r; Verrucaria cf. calciseda X16: r; Xanthoria parietina X16: r; Epixyle Flechten: Hypogymnia physodes } \\
\text { T09: r }\end{array}$} \\
\hline
\end{tabular}




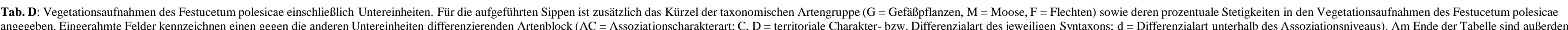
angegeben. Eingerahmte
die Kopfdaten aufgelistet

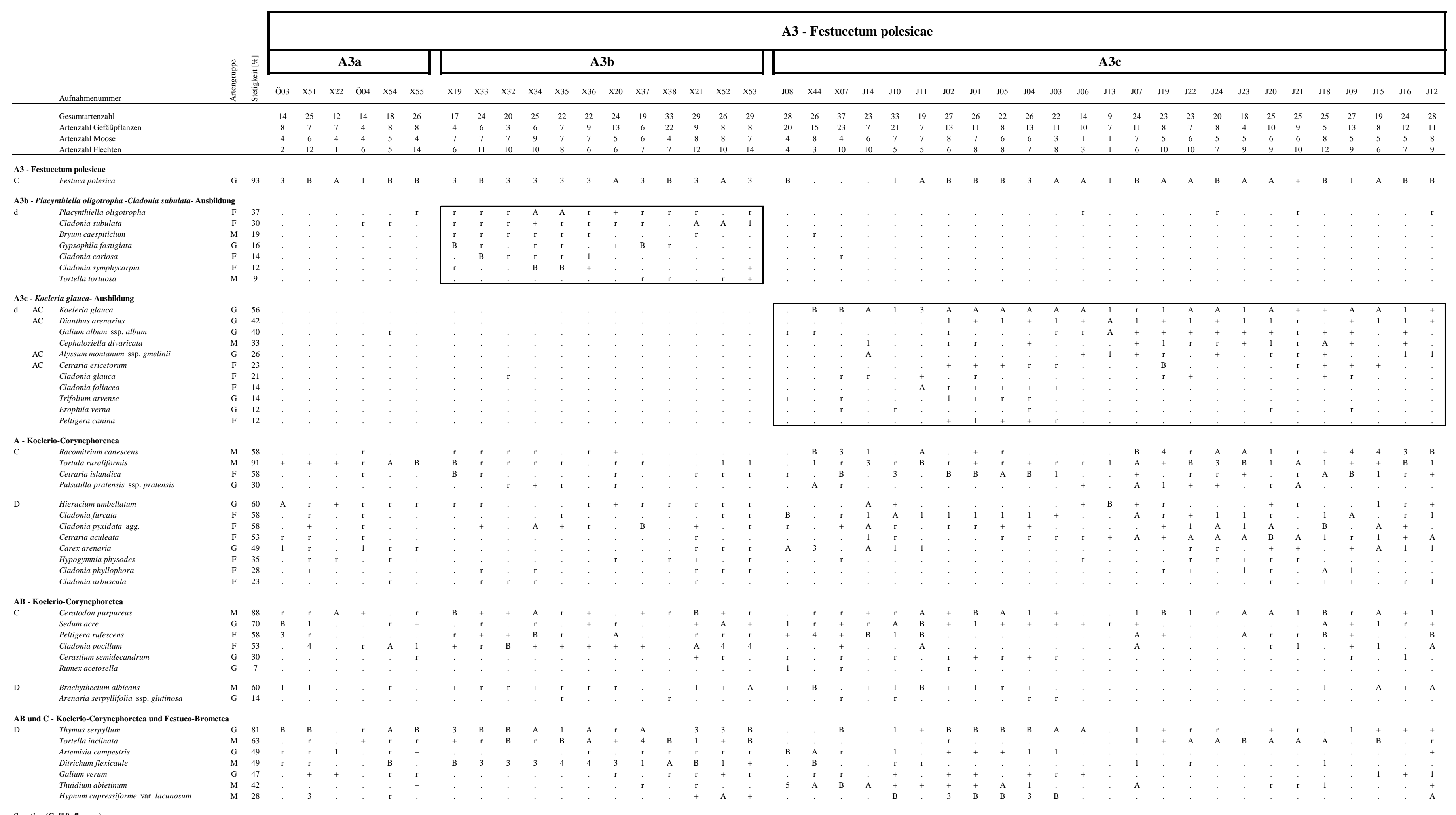




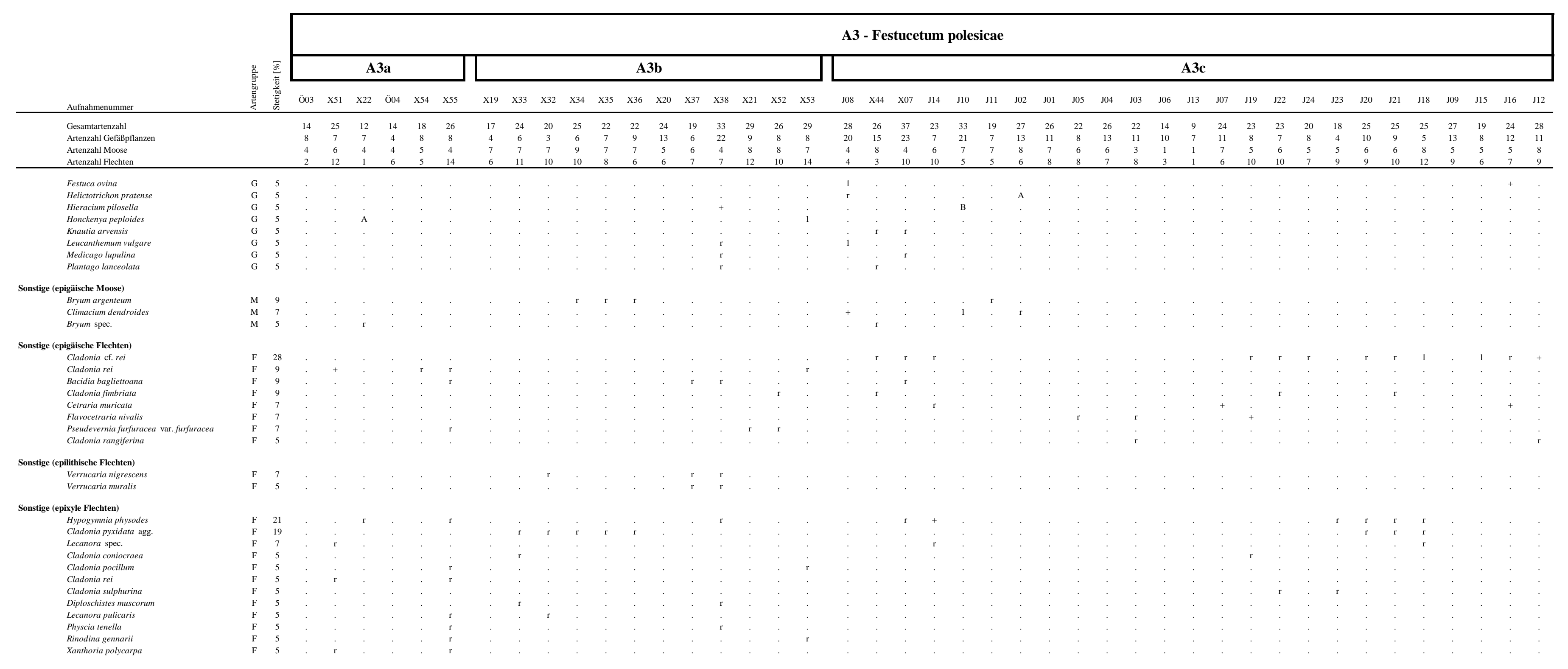

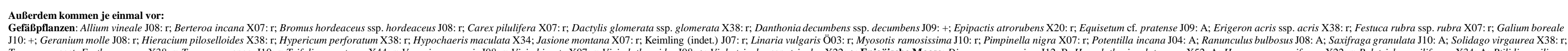

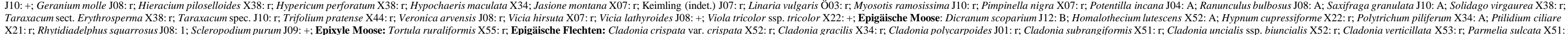

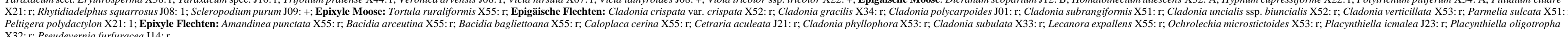




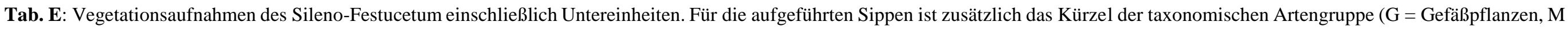

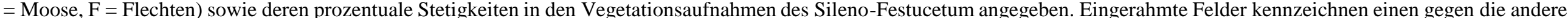

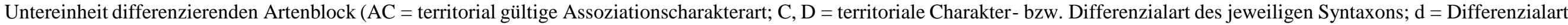

unterhalb des Assoziationsniveaus; $*=$ Zentralsyntaxon). Am Ende der Tabelle sind außerdem die Kopfdaten aufgelistet.

Aufnahmenummer

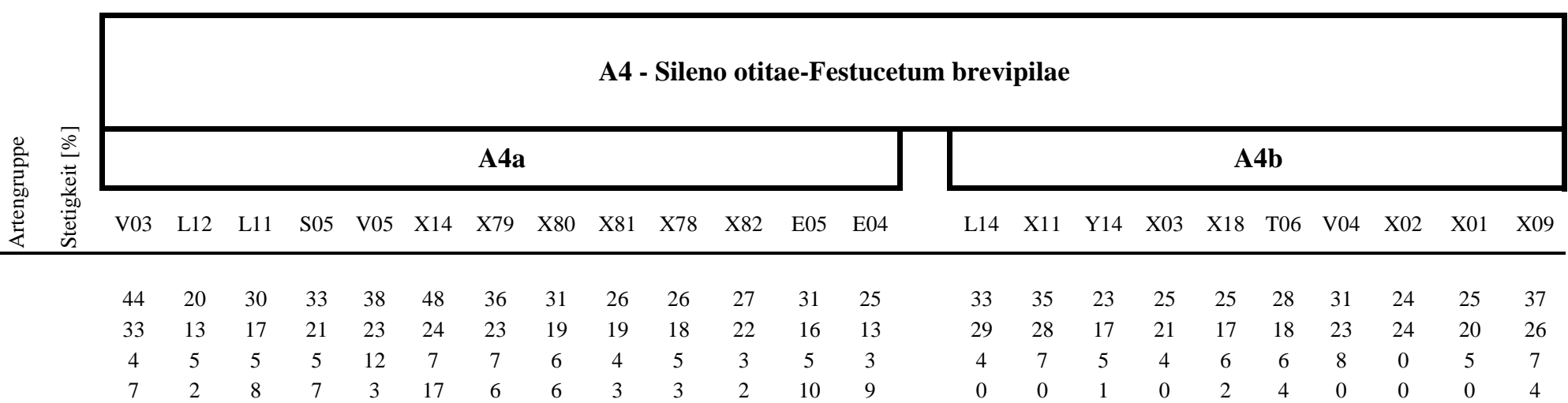

A4 - Sileno otitae-Festucetum brevipilae *

C Trifolium arvense

Polytrichum juniperinum

Dianthus deltoides

Vicia lathyroides

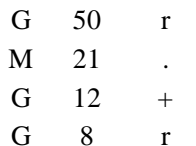

A4a - Cladonia furcata -Helictotrichon pratense -Ausbildung

d Helictotrichon pratense

Cladonia furcata

Climacium dendroides

Homalothecium lutescens

Cladonia pyxidata agg.

Hypnum cupressiforme var. lacunosum

Pimpinella nigra

Carex pilulifera

Cetraria islandica

AC Phleum phleoides

Cladonia arbuscula

Cladonia rangiformis

Dicranum scoparium

Peltigera canina

Potentilla incana

Pseudolysimachion spicatum

Cladonia subrangiformis

Asperula tinctoria

Carex caryophyllea

Carex ericetorum

Cetraria aculeata

Cladonia rei

\begin{tabular}{lll|ccccccccccccc|}
$\mathrm{G}$ & 58 & 5 & + & 1 & $\mathrm{r}$ & + & $\mathrm{A}$ & + & $\mathrm{B}$ & 3 & $\mathrm{~B}$ & 3 & 4 & $\mathrm{~B}$ & $\mathrm{~B}$ \\
$\mathrm{~F}$ & 42 & $\mathrm{~A}$ & $\mathrm{~B}$ & $\mathrm{~A}$ & $\mathrm{~A}$ & $\mathrm{~A}$ &. & $\mathrm{~B}$ & $\mathrm{~A}$ & + & $\mathrm{B}$ & + &. &. \\
$\mathrm{M}$ & 42 & $\mathrm{r}$ & $\mathrm{r}$ &. & $\mathrm{B}$ &. &. & 1 & 1 & $\mathrm{~A}$ & $\mathrm{r}$ & 1 &. &. \\
$\mathrm{M}$ & 42 &. & 3 & $\mathrm{r}$ & $\mathrm{A}$ &. & $\mathrm{B}$ & 3 & 3 & $\mathrm{~A}$ & 3 & 3 &. &. \\
$\mathrm{~F}$ & 38 & + &. & $\mathrm{A}$ &. &. & $\mathrm{r}$ & $\mathrm{r}$ & $\mathrm{r}$ & $\mathrm{r}$ & $\mathrm{r}$ & $\mathrm{r}$ &. &. \\
$\mathrm{M}$ & 38 & 3 &. &. &. & $\mathrm{~A}$ & 3 & $\mathrm{~A}$ & $\mathrm{~A}$ &. & $\mathrm{r}$ &. & $\mathrm{B}$ & 4 \\
$\mathrm{G}$ & 33 & $\mathrm{r}$ &. & $\mathrm{r}$ & $\mathrm{r}$ &. & $\mathrm{A}$ &. & $\mathrm{r}$ & $\mathrm{r}$ &. & $\mathrm{r}$ &. &. \\
$\mathrm{G}$ & 25 &. &. &. &. &. &. & $\mathrm{r}$ & + & $\mathrm{r}$ & $\mathrm{r}$ & $\mathrm{r}$ &. &. \\
$\mathrm{F}$ & 25 & 1 &. &. &. &. & $\mathrm{r}$ & $\mathrm{r}$ & $\mathrm{r}$ &. &. &. & $\mathrm{B}$ & 1 \\
$\mathrm{G}$ & 25 & 1 & $\mathrm{~B}$ & $\mathrm{r}$ &. &. &. & + &. &. &. &. &. &. &. \\
$\mathrm{F}$ & 21 &. &. &. & $\mathrm{~A}$ & $\mathrm{~B}$ &. &. &. &. &. &. & $\mathrm{r}$ & $\mathrm{r}$ \\
$\mathrm{F}$ & 21 & + &. &. &. &. & $\mathrm{r}$ & $\mathrm{r}$ & $\mathrm{A}$ &. &. &. &. & $\mathrm{r}$ &. \\
$\mathrm{M}$ & 21 & 4 &. &. & 3 & $\mathrm{~A}$ &. & $\mathrm{~A}$ & + &. &. &. &. &. \\
$\mathrm{F}$ & 21 & + & $\mathrm{B}$ &. &. &. &. & $\mathrm{r}$ & $\mathrm{r}$ & $\mathrm{r}$ &. &. &. &. \\
$\mathrm{G}$ & 21 &. &. &. &. &. &. &. & $\mathrm{r}$ &. & $\mathrm{r}$ & $\mathrm{r}$ & $\mathrm{B}$ & $\mathrm{A}$ \\
$\mathrm{G}$ & 21 &. &. &. &. &. &. & + & $\mathrm{A}$ & $\mathrm{B}$ & 1 & + &. &. \\
$\mathrm{F}$ & 17 &. &. & + &. &. &. & $\mathrm{r}$ &. &. &. &. & 1 & $\mathrm{~A}$ \\
$\mathrm{G}$ & 12 & $\mathrm{~A}$ &. &. &. &. &. &. &. &. & $\mathrm{r}$ & + &. &. \\
$\mathrm{G}$ & 12 & $\mathrm{~B}$ & 1 &. &. &. &. & $\mathrm{r}$ &. &. &. &. &. &. \\
$\mathrm{G}$ & 12 & + &. &. & + &. &. &. &. &. &. &. &. & $\mathrm{A}$ \\
$\mathrm{F}$ & 12 &. &. & $\mathrm{r}$ &. &. &. &. &. &. &. &. & $\mathrm{r}$ & $\mathrm{r}$ \\
$\mathrm{F}$ & 12 &. &. &. &. &. & $\mathrm{r}$ &. &. &. &. &. & $\mathrm{r}$ & $\mathrm{r}$
\end{tabular}


A4b - Potentilla argentea -Agrostis capillaris -Ausbildung

Potentilla argentea agg.

Agrostis capillaris

Hypericum perforatum

Rhytidiadelphus squarrosus

Rumex acetosella

Bromus hordeaceus ssp. hordeaceus

Dactylis glomerata ssp. glomerata

Ranunculus bulbosus

Rumex acetosa

Senecio jacobaea ssp. jacobaea

Trifolium repens

Viola arvensis ssp. arvensis

Galium album ssp. album

Phleum bertolonii

Pinus sylvestris ssp. sylvestris (juv.)

$\begin{array}{ll}\text { G } & 38 \\ \text { G } & 33 \\ \text { G } & 29 \\ \text { M } & 25 \\ \text { G } & 21 \\ \text { G } & 17 \\ \text { G } & 17 \\ \text { G } & 17 \\ \text { G } & 17 \\ \text { G } & 17 \\ \text { G } & 17 \\ \text { G } & 17 \\ \text { G } & 12 \\ \text { G } & 12 \\ \text { G } & 12\end{array}$

$\begin{array}{ccccccccccccc}44 & 20 & 30 & 33 & 38 & 48 & 36 & 31 & 26 & 26 & 27 & 31 & 25 \\ 33 & 13 & 17 & 21 & 23 & 24 & 23 & 19 & 19 & 18 & 22 & 16 & 13 \\ 4 & 5 & 5 & 5 & 12 & 7 & 7 & 6 & 4 & 5 & 3 & 5 & 3\end{array}$

$\begin{array}{llllllllll}33 & 35 & 23 & 25 & 25 & 28 & 31 & 24 & 25 & 37 \\ 29 & 28 & 17 & 21 & 17 & 18 & 23 & 24 & 20 & 26\end{array}$

$\begin{array}{lllllllllllll}7 & 2 & 8 & 7 & 3 & 17 & 6 & 6 & 3 & 3 & 2 & 10 & 9\end{array}$

$\begin{array}{cccccccccc}4 & 7 & 5 & 4 & 6 & 6 & 8 & 0 & 5 & 7\end{array}$

\section{folio arvensis-Festucetalia ovinae}

Myosotis stricta

D Festuca ovina

Poa angustifolic

Vicia hirsuta

Luzula campestris

Medicago lupulina

Anthyllis vulneraria ssp. vulneraria

Helictotrichon pubescens ssp. pubescens

Hieracium pilosella

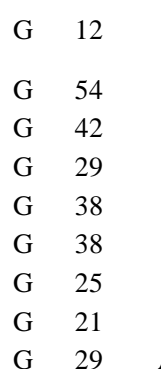

\section{A - Koelerio-Corynephorenea}

Pulsatilla pratensis ssp. pratensis

Tortula ruraliformis

D Carex arenaria

Hieracium umbellatum

$+$

\begin{tabular}{|c|c|c|c|c|c|c|c|c|c|}
\hline $\mathrm{r}$ & $\mathrm{r}$ & 1 & . & + & A & . & $\mathrm{r}$ & 1 & r \\
\hline A & 4 & . & $\mathrm{r}$ & $\mathrm{r}$ & A & . & A & . & . \\
\hline $\mathrm{r}$ & $\mathrm{r}$ & . & $\mathrm{r}$ & $\mathrm{r}$ & . & . & . & . & $\mathrm{r}$ \\
\hline B & $\mathrm{r}$ & $\mathrm{r}$ & . & A & . & A & . & . & . \\
\hline+ & A & . & $\mathrm{r}$ & & $\mathrm{r}$ & . & . & . & . \\
\hline$\cdot$ & $\cdot$ & . & . & & $\cdot$ & 1 & $\mathrm{r}$ & 1 & . \\
\hline . & . & $\mathrm{r}$ & . & . & . & $\mathrm{r}$ & 1 & - & . \\
\hline $\mathrm{r}$ & . & $\cdot$ & . & + & . & $\mathrm{r}$ & . & . & . \\
\hline 1 & $\mathrm{r}$ & . & . & . & $\cdot$ & $\cdot$ & $\cdot$ & . & $\mathrm{r}$ \\
\hline$\cdot$ & $\mathrm{r}$ & . & . & $\mathrm{r}$ & $\cdot$ & $\cdot$ & $\cdot$ & . & . \\
\hline 1 & $\mathrm{r}$ & . & . & r. & . & $\mathrm{r}$ & $\mathrm{r}$ & . & . \\
\hline$\cdot$ & $\mathrm{r}$ & & . & $\cdot$ & & $\mathrm{r}$ & & $\mathrm{r}$ & . \\
\hline & $\cdot$ & + & . & & · & $\cdot$ & . & + & . \\
\hline B & + & . & . & & · & $\cdot$ & $\mathrm{r}$ & . & . \\
\hline & 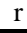 & . & $\mathrm{r}$ & & . & . & . & . & $\mathrm{r}$ \\
\hline
\end{tabular}


Hypogymnia physodes

Placynthiella oligotropha

$\begin{array}{ccccccccccccc}44 & 20 & 30 & 33 & 38 & 48 & 36 & 31 & 26 & 26 & 27 & 31 & 25 \\ 33 & 13 & 17 & 21 & 23 & 24 & 23 & 19 & 19 & 18 & 22 & 16 & 13 \\ 4 & 5 & 5 & 5 & 12 & 7 & 7 & 6 & 4 & 5 & 3 & 5 & 3 \\ 7 & 2 & 8 & 7 & 3 & 17 & 6 & 6 & 3 & 3 & 2 & 10 & 9\end{array}$

$\begin{array}{ccc}33 & 35 & 23 \\ 29 & 28 & 17 \\ 4 & 7 & 5\end{array}$

\begin{tabular}{cccccccc}
3 & 25 & 25 & 28 & 31 & 24 & 25 & 37 \\
7 & 21 & 17 & 18 & 23 & 24 & 20 & 26 \\
5 & 4 & 6 & 6 & 8 & 0 & 5 & 7 \\
1 & 0 & 2 & 4 & 0 & 0 & 0 & 4 \\
\hline
\end{tabular}

\section{AB - Koelerio-Corynephoretea}

C Galium verum

Cerastium semidecandrum

Ceratodon purpureus

Peltigera rufescens

Cladonia pocillum

D Arenaria serpyllifolia ssp. glutinosa Brachythecium albican

C - Koelerio-Corynephoretea und Festuco-Bromete

D Artemisia campestris

Sedum acre

Thuidium abietinum

Thymus serpyllum

$\begin{array}{ll}\text { G } & 67 \\ \text { G } & 46 \\ \text { M } & 38 \\ \text { F } & 33 \\ \text { F } & 17 \\ \text { G } & 62 \\ \text { M } & 46\end{array}$

\section{(Gefäßpflanzen)}

Achillea millefolium ssp. millefolium

Plantago lanceolata

Festuca rubra ssp. rubra

Elymus repens ssp. repens

Vicia tetrasperma

Potentilla tabernaemontani

Potentilla $\times$ subarenaria

Silene nutans

Arabis hirsuta

Acinos arvensis

Veronica officinalis

Convolvulus arvensis

Herniaria glabra

Knautia arvensis

Pimpinella saxifraga

Poa compressa

$\begin{array}{lllllllllllllll}\mathrm{G} & 75 & \mathrm{r} & . & + & \mathrm{r} & + & \mathrm{B} & \mathrm{r} & 1 & \mathrm{r} & \mathrm{r} & \mathrm{r} & \mathrm{B} & \mathrm{A} \\ \mathrm{G} & 71 & \mathrm{r} & \mathrm{B} & 1 & \mathrm{r} & + & 1 & 1 & + & 1 & + & + & \mathrm{r} & + \\ \mathrm{M} & 62 & + & 5 & \mathrm{~A} & \mathrm{r} & \mathrm{r} & 1 & 3 & 3 & 4 & 4 & 4 & . & . \\ \mathrm{G} & 62 & \mathrm{~A} & + & . & 1 & \mathrm{~B} & 1 & 4 & 3 & \mathrm{~A} & 3 & \mathrm{~B} & 1 & \mathrm{r}\end{array}$

Agrostis vinealis

$\begin{array}{ll}\text { G } & 71 \\ \text { G } & 62 \\ \text { G } & 38 \\ \text { G } & 33 \\ \text { G } & 29 \\ \text { G } & 17 \\ \text { G } & 17 \\ \text { G } & 17 \\ \text { G } & 21 \\ \text { G } & 17 \\ \text { G } & 17 \\ \text { G } & 12 \\ \text { G } & 12 \\ \text { G } & 12 \\ \text { G } & 12 \\ \text { G } & 12 \\ \text { G } & 8\end{array}$


Artenzahl Moose

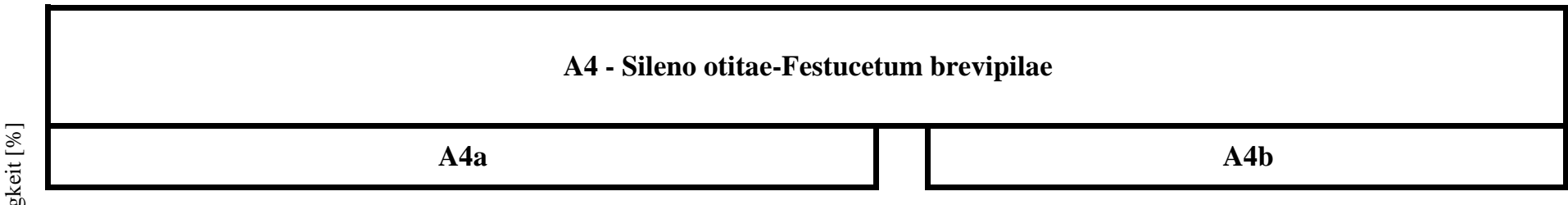

Artenzahl Flechten

$\begin{array}{lllllllllllll}\text { V03 } & \text { L12 } & \text { L11 } & \text { S05 } & \text { V05 } & \text { X14 } & \text { X79 } & \text { X80 } & \text { X81 } & \text { X78 } & \text { X82 } & \text { E05 } & \text { E04 }\end{array}$

$\begin{array}{llllllllll}\text { L14 } & \text { X11 } & \text { Y14 } & \text { X03 } & \text { X18 } & \text { T06 } & \text { V04 } & \text { X02 } & \text { X01 } & \text { X09 }\end{array}$

Arenaria serpyllifolia ssp. serpyllifolia

Arrhenatherum elatius

Campanula rotundifolia

Capsella bursa-pastoris

Carex ornithopoda ssp. ornithopoda

Cerastium arvense ssp. arvense

Cerastium holosteoides

Euphrasia stricta

Jasione montana

Leucanthemum vulgare

Linum catharticum

Potentilla reptans

Scleranthus annuus

Silene latifolia ssp. alba

Stellaria graminea

Taraxacum sect. Erythrosperma

Taraxacum sect. Ruderali

Trifolium dubium

Trifolium montanum

Trifolium pratense

Veronica arvensis

Veronica chamaedrys ssp. chamaedrys

\begin{tabular}{cccccccccccc}
44 & 20 & 30 & 33 & 38 & 48 & 36 & 31 & 26 & 26 & 27 & 31 \\
33 & 13 & 17 & 21 & 23 & 24 & 23 & 19 & 19 & 18 & 22 & 16 \\
4 & 5 & 5 & 5 & 12 & 7 & 7 & 6 & 4 & 5 & 3 & 5 \\
7 & 2 & 8 & 7 & 3 & 17 & 6 & 6 & 3 & 3 & 2 & 10 \\
\hline
\end{tabular}

$\begin{array}{cccccccccc}33 & 35 & 23 & 25 & 25 & 28 & 31 & 24 & 25 & 37 \\ 29 & 28 & 17 & 21 & 17 & 18 & 23 & 24 & 20 & 26 \\ 4 & 7 & 5 & 4 & 6 & 6 & 8 & 0 & 5 & 7 \\ 0 & 0 & 1 & 0 & 2 & 4 & 0 & 0 & 0 & 4\end{array}$

Sonstige (epigäische Moose)

Ditrichum flexicaule

Bryum caespiticium

Bryum spec.

Pleurozium schreberi

Racomitrium canescens

Barbula unguiculata

Bryum argenteum

Funaria hygrometrica

Plagiomnium affine

Rhodobryum roseum

Thuidium philibertii

Tortella inclinata
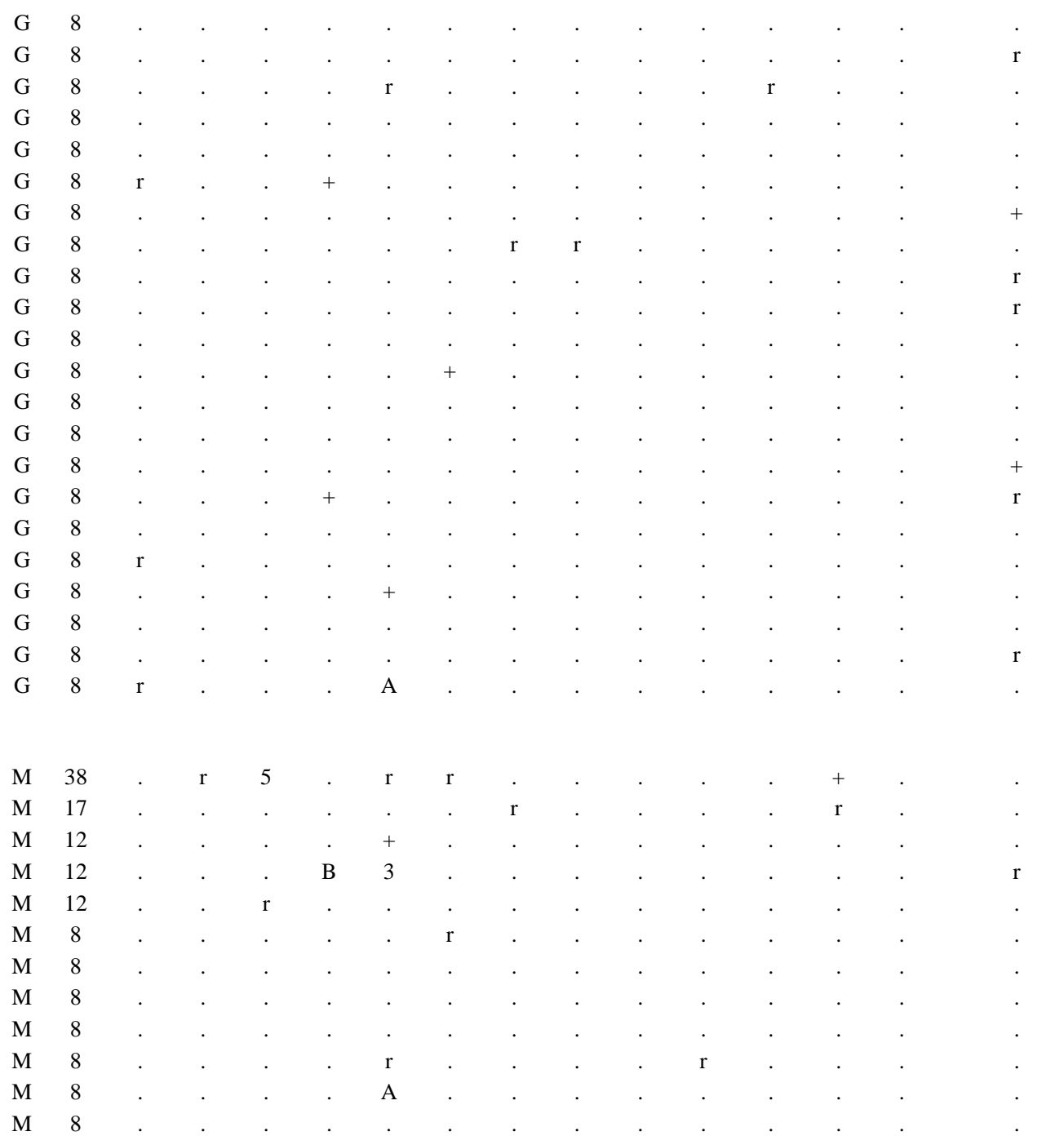
Cladonia fimbriat

Cladonia symphycarpia

\section{Sonstige (epilithische Flechten)} Verrucaria nigrescens

Verrucaria muralis

$\begin{array}{ccccccccccccc}44 & 20 & 30 & 33 & 38 & 48 & 36 & 31 & 26 & 26 & 27 & 31 & 25 \\ 33 & 13 & 17 & 21 & 23 & 24 & 23 & 19 & 19 & 18 & 22 & 16 & 13 \\ 4 & 5 & 5 & 5 & 12 & 7 & 7 & 6 & 4 & 5 & 3 & 5 & 3 \\ 7 & 2 & 8 & 7 & 3 & 17 & 6 & 6 & 3 & 3 & 2 & 10 & 9\end{array}$

\begin{tabular}{cccccccccc}
33 & 35 & 23 & 25 & 25 & 28 & 31 & 24 & 25 & 37 \\
29 & 28 & 17 & 21 & 17 & 18 & 23 & 24 & 20 & 26 \\
4 & 7 & 5 & 4 & 6 & 6 & 8 & 0 & 5 & 7 \\
0 & 0 & 1 & 0 & 2 & 4 & 0 & 0 & 0 & 4 \\
\hline
\end{tabular}

\section{Sonstige (epixyle Flechten)} Hypogymnia physodes

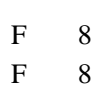

$\begin{array}{ll}\mathrm{F} & 8 \\ \mathrm{~F} & 8\end{array}$

\section{ußerdem kommen je einmal vor:}

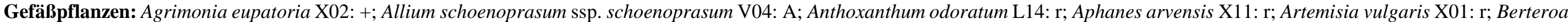

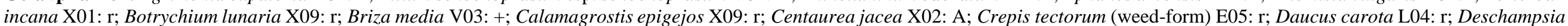

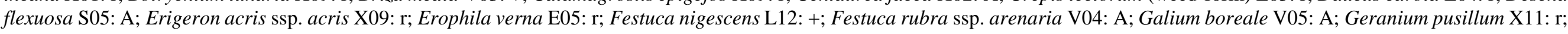

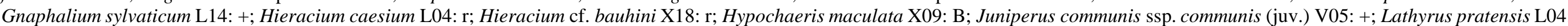

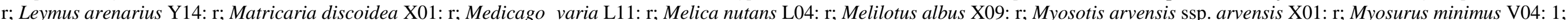

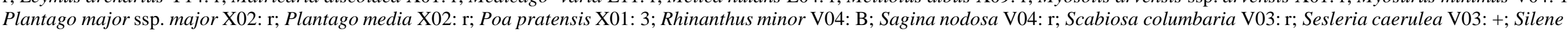

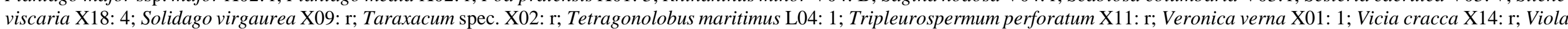

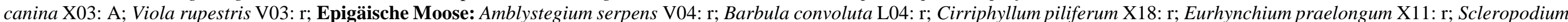

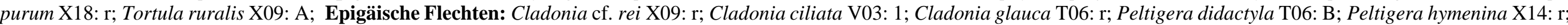

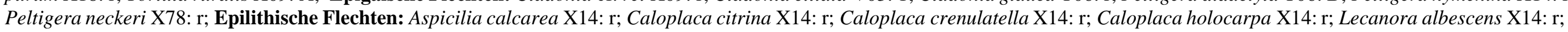

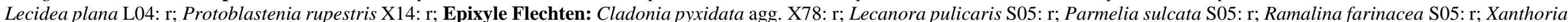
parietina L11: r; Xanthoria polycarpa X14: r; Epiphytische Flechten: Hypogymnia physodes V05: r 
Tab. F: Vegetationsaufnahmen des Cladonio-Sedetum einschließlich Untereinheiten. Für die aufgeführten Sippen ist zusätzlich das Kürzel der taxonomischen Artengruppe $(\mathrm{G}=$ Gefäßpflanzen, $\mathrm{M}=$ Moose, $\mathrm{F}=$ Flechten) sowie deren prozentuale Stetigkeiten in den Vegetationsaufnahmen des Cladonio-Sedetum angegeben. Eingerahmte Felder kennzeichnen einen gegen die andere Untereinheit differenzierenden Artenblock $(C, D=$ territoriale Charakter- bzw. Differenzialart des jeweiligen Syntaxons; $d=$

Differenzialart unterhalb des Assoziationsniveaus; * = Zentralsyntaxon). Am Ende der Tabelle sind außerdem die Kopfdaten aufgelistet.

\begin{tabular}{|c|c|c|c|c|c|c|c|c|c|c|c|c|c|c|c|c|c|c|c|c|c|c|c|c|c|}
\hline \multirow[b]{3}{*}{ Aufnahmenummer } & \multirow{3}{*}{ 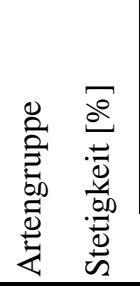 } & \multicolumn{24}{|c|}{ B1 - Cladonio symphycarpae-Sedetum albi } \\
\hline & & \multicolumn{14}{|c|}{ B1a } & \multicolumn{10}{|c|}{ B1b } \\
\hline & & N01 & N02 & $\mathrm{X} 30$ & $\mathrm{X} 23$ & $\mathrm{X} 42$ & $\mathrm{X} 412$ & $\mathrm{X} 432$ & X39 & $\mathrm{X} 40$ & $\mathrm{X} 45$ & P04 & P05 & $\mathrm{X} 12$ & X77 & T14 & K01 & $\mathrm{X} 67$ & V07 & T04 & L10 & $\mathrm{X} 24$ & $\mathrm{X} 25 \mathrm{Y} 01$ & K02 & K03 \\
\hline Gesamtartenzahl & & 26 & 22 & 27 & 24 & 42 & 39 & 33 & 45 & 43 & 36 & 34 & 32 & 35 & 32 & 21 & 33 & 24 & 28 & 21 & 45 & 39 & 20 & 16 & 30 \\
\hline Artenzahl Gefäßpflanzen & & 18 & 15 & 21 & 14 & 26 & 24 & 20 & 19 & 23 & 16 & 24 & 21 & 19 & 19 & 18 & 20 & 15 & 11 & 13 & 35 & 17 & 20 & 11 & 13 \\
\hline Artenzahl Moose & & 7 & 6 & 6 & 8 & 11 & 10 & 7 & 9 & 8 & 8 & 5 & 5 & 12 & 8 & 3 & 9 & 4 & 5 & 7 & 8 & 10 & 13 & 4 & 8 \\
\hline
\end{tabular}

B1 - Cladonio sympycarpae-Sedetum albi

C Saxifraga tridactylites

D Brachythecium albicans

Bromus hordeaceus ssp. hordeaceus

Arenaria serpyllifolia ssp. serpyllifolia

G 28 A 3

$\begin{array}{llllll}\mathrm{M} & 60 & 1 & \mathrm{~A} & \mathrm{~A}+\end{array}$

$\begin{array}{lllllll}\mathrm{G} & 36 & + & \mathrm{r} & \mathrm{r} & \mathrm{A} \\ \mathrm{G} & 32 & 1 & 1 & . & \mathrm{B}\end{array}$

B1a - Artemisia campestris -Bryum caespiticum -Ausbildung

d Artemisia campestris

Bryum caespitici

Dactylis glomerata ssp. glomerata

Poa compressa

Acinos arvensis

Peltigera rufescens

Plantago lanceolata

Potentilla argentea agg.

Poa angustifolia

Bryum argenteum

Knautia arvensis

Tortula ruralis

Cladonia novochlorophaea

Phleum bertolonii

Trifolium arvense

Vicia hirsuta

Galium album ssp. album

Herniaria glabra

Linaria vulgaris

Melampyrum arvense

Peltigera canina

Anthemis tinctoria

Cladonia fimbriata

Rhytidiadelphus squarrosus

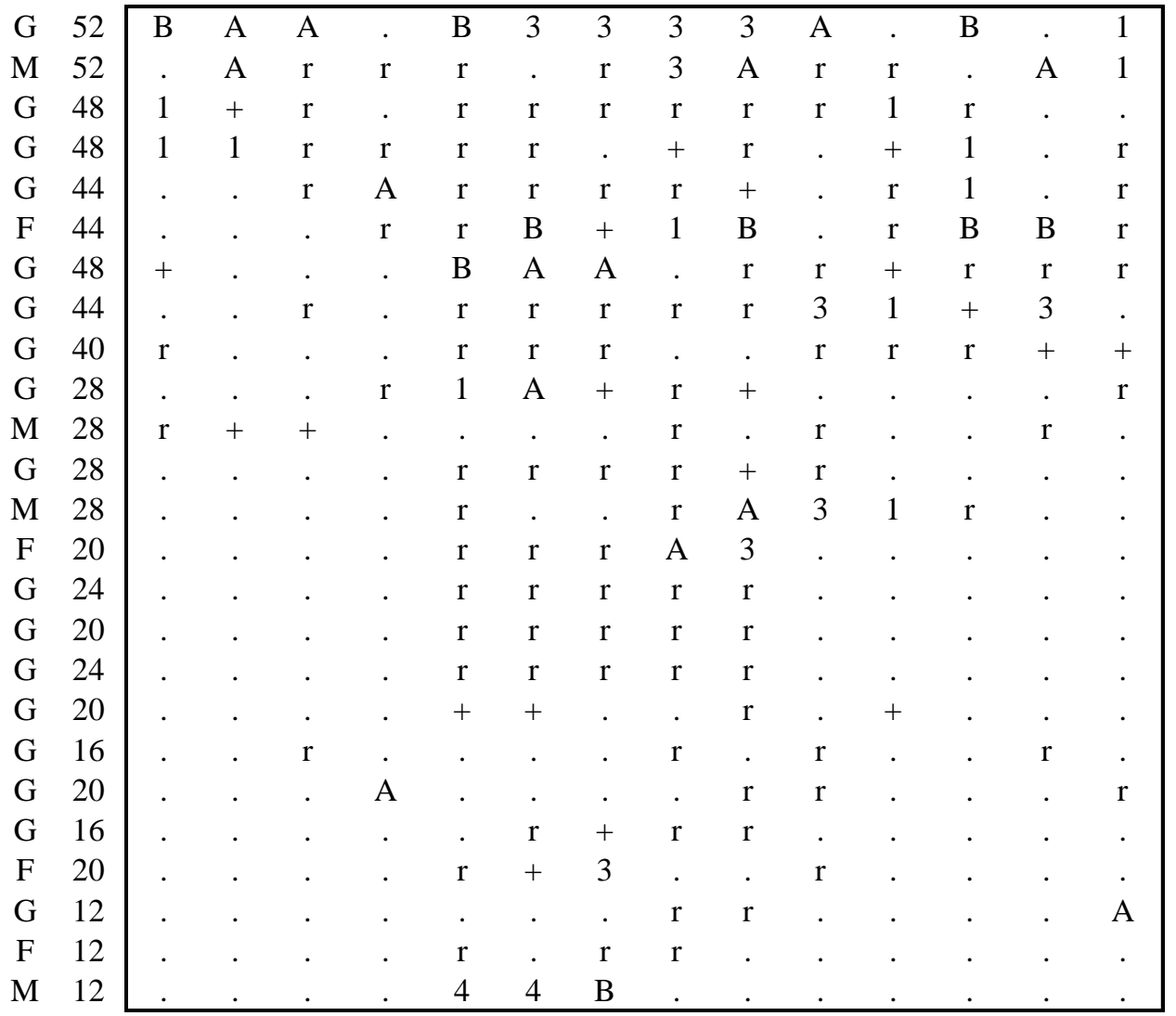

B1b - Tortella tortuosa -Leontodon autumnalis -Ausbildung 


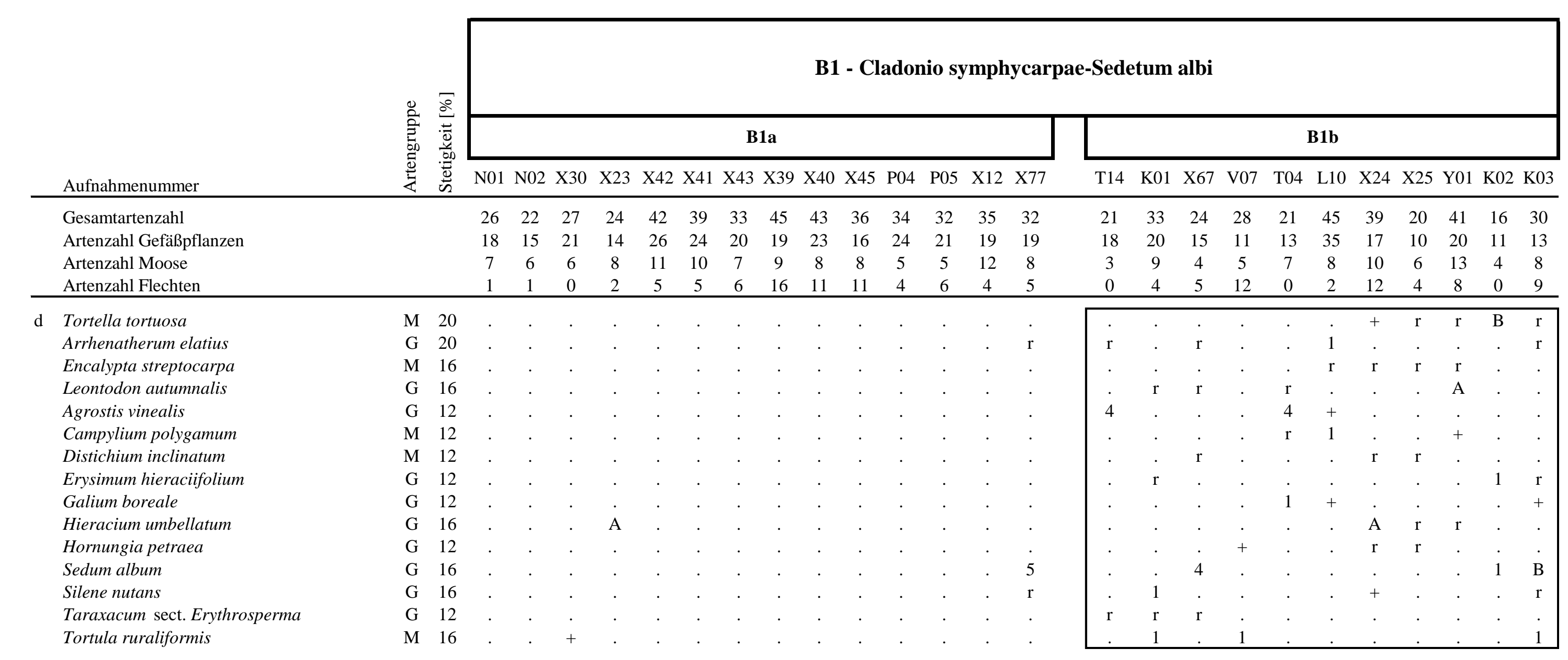

\section{B.a1a Tortello tortuosae-Sedenion albi *}

\section{Daucus carota \\ G $20 \quad 1$ +}

Erophila verna

B.a1 Tortello tortuosae-Sedion albi

C Cladonia symphycarpia

Barbula unguiculata

D Homalothecium lutescens

Medicago lupulina

Arenaria serpyllifolia ssp. glutinosa

Anthyllis vulneraria ssp. vulneraria

Hypericum perforatum

Nostoc spec.

Ranunculus bulbosus

Sagina nodosa

Pimpinella nigra

Agrostis stolonifera

Cladonia rangiformis

G $32 \quad \mathrm{r}$

\section{B - Sedo-Scleranthenea}

D Echium vulgare

Barbula convoluta

Festuca ovina

Pseudolysimachion spicatum

Fissidens dubius

Potentilla tabernaemontani 


\begin{tabular}{|c|c|c|c|c|c|c|c|c|c|c|c|c|c|c|c|c|c|c|c|c|c|c|c|c|c|c|}
\hline \multirow[b]{3}{*}{ Aufnahmenummer } & \multirow{3}{*}{ 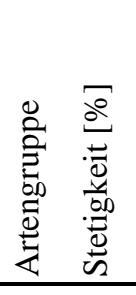 } & \multicolumn{25}{|c|}{ B1 - Cladonio symphycarpae-Sedetum albi } \\
\hline & & \multicolumn{14}{|c|}{ B1a } & \multicolumn{11}{|c|}{ B1b } \\
\hline & & N01 & N02 & $\mathrm{X} 30$ & $\mathrm{X} 23$ & $\mathrm{X} 42$ & $\mathrm{X} 41$ & $\mathrm{X} 43$ & $\mathrm{X} 39$ & $\mathrm{X} 40$ & $\mathrm{X} 45$ & P04 & P05 & $\mathrm{X} 12$ & X77 & T14 & K01 & $\mathrm{X} 67$ & V07 & T04 & L10 & $\mathrm{X} 24$ & $\mathrm{X} 25$ & Y01 & K02 & K03 \\
\hline Gesamtartenzahl & & 26 & 22 & 27 & 24 & 42 & 39 & 33 & 45 & 43 & 36 & 34 & 32 & 35 & 32 & 21 & 33 & 24 & 28 & 21 & 45 & 39 & 20 & 41 & 16 & 30 \\
\hline Artenzahl Gefäßpflanzen & & 18 & 15 & 21 & 14 & 26 & 24 & 20 & 19 & 23 & 16 & 24 & 21 & 19 & 19 & 18 & 20 & 15 & 11 & 13 & 35 & 17 & 10 & 20 & 11 & 13 \\
\hline Artenzahl Moose & & 7 & 6 & 6 & 8 & 11 & 10 & 7 & 9 & 8 & 8 & 5 & 5 & 12 & 8 & 3 & 9 & 4 & 5 & 7 & 8 & 10 & 6 & 13 & 4 & 8 \\
\hline Artenzahl Flechten & & 1 & 1 & 0 & 2 & 5 & 5 & 6 & 16 & 11 & 11 & 4 & 6 & 4 & 5 & 0 & 4 & 5 & 12 & 0 & 2 & 12 & 4 & 8 & 0 & 9 \\
\hline
\end{tabular}

AB - Koelerio-Corynephoretea

$\begin{array}{lcccccccccccccccccc}\text { C Sedum acre } & \text { G } & 88 & \text { B } & 1 & \text { B } & \text { A } & + & \text { A } & \text { B } & \text { A } & \text { A } & 3 & 1 & \text { A } & \text { B } & \text { A } \\ \quad \text { Ceratodon purpureus } & \text { M } & 68 & \text { r } & \text { r } & 3 & \text { r } & \text { r } & \text { r } & + & \text { B } & 1 & . & 3 & 3 & + & +\end{array}$

Cerastium semidecandrum

$\begin{array}{lllllllllllllllll}M & 68 & \mathrm{r} & \mathrm{r} & 3 & \mathrm{r} & \mathrm{r} & \mathrm{r} & + & \mathrm{B} & 1 & . & 3 & 3 & + & + \\ \mathrm{G} & 36 & \mathrm{r} & + & \mathrm{r} & \mathrm{A} & . & . & . & . & . & \mathrm{r} & 1 & \mathrm{~A} & . & .\end{array}$

AB und C - Koelerio-Corynephoretea und Festuco-Brometea

D Ditrichum flexicaule

Thuidium abietinum

Tortella inclinata

Galium verum

M $84+$

Hypnum cupressiforme var. lacunosum

M 48

G $\quad 32$

M 32

\section{Sonstige (Gefäßpflanzen)}

Achillea millefolium ssp. millefolium

Festuca rubra ssp. rubra

Hieracium pilosella

Elymus repens ssp. repens

Centaurea jacea

Arabis hirsuta

Crepis tectorum ("weed-form")

Taraxacum spec.

Solidago virgaurea

Veronica arvensis

Campanula rotundifolia

Cardamine hirsuta

Carlina vulgaris

Centaurea scabiosa ssp. scabiosa

Erigeron acris ssp. acris

Fragaria viridis

Linum catharticum

Lotus corniculatus ssp. corniculatus

Medicago falcata

Plantago media

Potentilla reptans

Senecio jacobaea ssp. jacobaea

Taraxacum sect. Ruderalia

Trifolium montanum

Trifolium repens

Valerianella locusta

Vicia cracca

$\begin{array}{ll}\mathrm{G} & 48 \\ \mathrm{G} & 32\end{array}$

G 32

G 28

G 24

G 16

G 12

G $\quad 12$

G 12

$\begin{array}{ll}\mathrm{G} & 12 \\ \mathrm{G} & 12\end{array}$

$\begin{array}{ll}\text { G } & 12 \\ \text { G } & 12\end{array}$

$\begin{array}{ll}\mathrm{G} & 12 \\ \mathrm{G} & 8\end{array}$

$\begin{array}{ll}\text { G } & 8 \\ \text { G } & 8\end{array}$

G 8

G 8

$\begin{array}{ll}\mathrm{G} & 8 \\ \mathrm{G} & 8\end{array}$

$\begin{array}{ll}\text { G } & 8 \\ \text { G } & 8\end{array}$

G 8

G 8

G 8

G 8

G 8

G 8

G 8

G 8

$\begin{array}{ll}\mathrm{G} & 8 \\ \mathrm{G} & 8\end{array}$

G 8

$\begin{array}{ll}\text { G } & 8 \\ \text { G } & 8\end{array}$ 


\begin{tabular}{|c|c|c|c|c|c|c|c|c|c|c|c|c|c|c|c|c|c|c|c|c|c|c|c|c|c|c|c|}
\hline \multirow[b]{2}{*}{ Aufnahmenummer } & \multirow{2}{*}{ 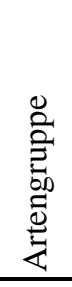 } & \multirow{2}{*}{ 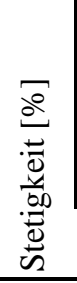 } & \multicolumn{25}{|c|}{ B1 - Cladonio symphycarpae-Sedetum albi } \\
\hline & & & No1 & $\mathrm{N} 02$ & X 30 & $\mathrm{X} 23$ & $X 42$ & $x 41$ & $X 43$ & X39 & $X 40$ & $x 45$ & P04 & P05 & $\mathrm{X} 12$ & $\mathrm{X} 77$ & T14 & K01 & X67 & V07 & T04 & - L10 & $\mathrm{X} 24$ & $\mathrm{X} 25$ & Y01 & K02 & K03 \\
\hline Gesamtartenzahl & & & 26 & 22 & 27 & 24 & 42 & 39 & 33 & 45 & 43 & 36 & 34 & 32 & 35 & 32 & 21 & 33 & 24 & 28 & 21 & 45 & 39 & 20 & 41 & 16 & 30 \\
\hline Artenzahl Gefäßpflanzen & & & 18 & 15 & 21 & 14 & 26 & 24 & 20 & 19 & 23 & 16 & 24 & 21 & 19 & 19 & 18 & 20 & 15 & 11 & 13 & 35 & 17 & 10 & 20 & 11 & 13 \\
\hline Artenzahl Moose & & & 7 & 6 & 6 & 8 & 11 & 10 & 7 & 9 & 8 & 8 & 5 & 5 & 12 & 8 & 3 & 9 & 4 & 5 & 7 & 8 & 10 & 6 & 13 & 4 & 8 \\
\hline Artenzahl Flechten & & & 1 & 1 & 0 & 2 & 5 & 5 & 6 & 16 & 11 & 11 & 4 & 6 & 4 & 5 & 0 & 4 & 5 & 12 & 0 & 2 & 12 & 4 & 8 & 0 & 9 \\
\hline \multicolumn{28}{|l|}{ Sonstige (epigäische Moose) } \\
\hline Bryum spec. & $\mathrm{M}$ & 12 & . & . & . & . & . & . & & $\mathrm{r}$ & . & . & . & . & . & $\mathrm{r}$ & & & . & . & . & . & $\mathrm{r}$ & & & . & \\
\hline Climacium dendroides & $\mathrm{M}$ & 12 & . & . & . & . & . & . & $\mathrm{r}$ & . & . & . & . & . & . & . & & 1 & . & . & . & . & . & . & . & + & \\
\hline Brachythecium rutabulum & $\mathrm{M}$ & 8 & . & . & . & . & . & . & . & . & . & . & . & . & $\mathrm{r}$ & . & & . & . & . & . & . & . & . & $\mathrm{r}$ & . & . \\
\hline Bryum cf. caespiticum & $\mathrm{M}$ & 8 & $\mathrm{r}$ & . & . & . & . & . & . & . & . & . & . & . & . & . & A & . & . & . & . & . & . & . & . & . & . \\
\hline Hypnuт сирressiforme & $\mathrm{M}$ & 8 & . & . & . & . & . & . & . & . & $\mathrm{r}$ & . & . & . & $\mathrm{r}$ & . & . & . & . & . & . & . & . & . & . & . & . \\
\hline Plagiomnium affine & $\mathrm{M}$ & 8 & . & . & . & . & $\mathrm{r}$ & $\mathrm{r}$ & . & . & $\cdot$ & $\cdot$ & $\cdot$ & $\cdot$ & $\cdot$ & . & $\cdot$ & . & . & $\cdot$ & $\cdot$ & $\cdot$ & $\cdot$ & . & . & $\cdot$ & \\
\hline \multicolumn{28}{|l|}{ Sonstige (epilithische Moose) } \\
\hline Tortula muralis & M & 8 & $\cdot$ & $\cdot$ & $\cdot$ & $\cdot$ & $\cdot$ & $\cdot$ & $\cdot$ & . & $\mathrm{r}$ & $\cdot$ & . & $\cdot$ & $\cdot$ & . & . & . & $\cdot$ & $\mathrm{r}$ & . & $\cdot$ & $\cdot$ & . & . & $\cdot$ & . \\
\hline \multicolumn{28}{|l|}{ Sonstige (epigäische Flechten) } \\
\hline Bacidia bagliettoana & $\mathrm{F}$ & 24 & . & . & . & . & . & . & $\mathrm{r}$ & $\mathrm{r}$ & $\mathrm{r}$ & . & . & . & . & . & & & . & $\mathrm{r}$ & . & . & $\mathrm{r}$ & . & $\mathrm{r}$ & . & \\
\hline Cladonia pocillum & $\mathrm{F}$ & 8 & . & . & . & . & . & . & . & . & . & . & . & . & . & . & . & . & . & 1 & . & . & + & . & . & . & . \\
\hline Cladonia pyxidata agg. & $\mathrm{F}$ & 8 & . & . & . & $\mathrm{r}$ & . & . & . & . & . & . & . & . & . & . & . & . & . & $\mathrm{r}$ & . & . & . & . & . & . & . \\
\hline Cladonia rei & $\mathrm{F}$ & 8 & . & . & . & . & . & . & . & $\mathrm{r}$ & $\mathrm{r}$ & . & . & . & . & . & . & . & $\cdot$ & . & . & . & . & . & . & . & . \\
\hline Cladonia subulata & $\mathrm{F}$ & 8 & . & . & . & . & . & . & . & $\mathrm{r}$ & $\mathrm{r}$ & . & . & . & . & . & . & . & . & . & . & . & . & . & . & . & . \\
\hline Ramalina farinacea & $\mathrm{F}$ & 8 & $\cdot$ & 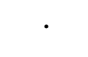 & $\cdot$ & $\cdot$ & $\cdot$ & $\cdot$ & $\cdot$ & $\cdot$ & $\mathrm{r}$ & $\mathrm{r}$ & $\cdot$ & $\cdot$ & $\cdot$ & . & & & $\cdot$ & $\cdot$ & $\cdot$ & $\cdot$ & $\cdot$ & . & . & $\cdot$ & \\
\hline \multicolumn{28}{|l|}{ Sonstige (epilithische Flechten) } \\
\hline Verrucaria nigrescens & $\mathrm{F}$ & 68 & . & . & . & . & . & $\mathrm{r}$ & $\mathrm{r}$ & $\mathrm{r}$ & $\mathrm{r}$ & $\mathrm{r}$ & + & + & $\mathrm{r}$ & + & . & + & $\mathrm{r}$ & B & . & 3 & $\mathrm{r}$ & B & $\mathrm{r}$ & . & 3 \\
\hline Verrucaria muralis & $\mathrm{F}$ & 52 & + & $\mathrm{r}$ & . & . & . & $\mathrm{r}$ & . & $\mathrm{r}$ & $\mathrm{r}$ & $\mathrm{r}$ & . & . & . & . & 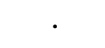 & . & $\mathrm{r}$ & A & . & + & $\mathrm{r}$ & B & A & . & B \\
\hline Protoblastenia rupestris & $\mathrm{F}$ & 32 & . & . & . & . & . & . & . & $\mathrm{r}$ & . & . & . & $\mathrm{r}$ & . & $\mathrm{r}$ & . & $\mathrm{r}$ & . & . & . & . & $\mathrm{r}$ & $\mathrm{r}$ & $\mathrm{r}$ & . & $\mathrm{r}$ \\
\hline Caloplaca crenulatella & $\mathrm{F}$ & 12 & . & . & . & . & . & . & . & . & . & . & . & . & . & . & & $\mathrm{r}$ & . & $\mathrm{r}$ & . & . & . & . & . & . & $\mathrm{r}$ \\
\hline Caloplaca holocarpa & $\mathrm{F}$ & 12 & . & . & . & . & . & . & . & . & . & . & . & . & . & . & & $\mathrm{r}$ & . & $\mathrm{r}$ & . & . & & & $\mathrm{r}$ & . & . \\
\hline Lecanora dispersa & $\mathrm{F}$ & 12 & . & . & . & . & . & . & . & . & . & . & . & . & . & . & & . & $\mathrm{r}$ & . & . & . & . & . & $\mathrm{r}$ & . & $\mathrm{r}$ \\
\hline Lecidella stigmatea & $\mathrm{F}$ & 12 & . & . & . & . & . & . & . & . & . & . & . & . & . & . & . & . & $\mathrm{r}$ & . & . & . & . & . & $\mathrm{r}$ & . & $\mathrm{r}$ \\
\hline Aspicilia calcarea & $\mathrm{F}$ & 8 & . & . & . & . & . & . & . & . & . & . & . & . & . & . & . & . & . & + & . & . & . & $\mathrm{r}$ & . & . & . \\
\hline Caloplaca citrina & $\mathrm{F}$ & 8 & . & . & . & . & . & . & . & $\mathrm{r}$ & . & . & . & . & . & . & . & . & . & . & . & . & . & . & . & . & $\mathrm{r}$ \\
\hline Caloplaca spec. & $\mathrm{F}$ & 8 & . & . & . & . & . & . & . & . & . & . & + & $\mathrm{r}$ & . & . & . & . & . & . & . & . & . & . & . & . & . \\
\hline Collema spec. & $\mathrm{F}$ & 8 & . & . & . & . & . & . & . & $\cdot$ & . & . & . & . & . & . & . & . & . & $\mathrm{r}$ & . & . & . & . & . & . & $\mathrm{r}$ \\
\hline \multicolumn{28}{|l|}{ Sonstige (epixyle Flechten) } \\
\hline Bacidia bagliettoana & $\mathrm{F}$ & 8 & . & . & . & . & . & . & $\mathrm{r}$ & . & . & . & . & . & . & . & . & & . & . & . & . & . & . & $\mathrm{r}$ & . & . \\
\hline Cladonia coniocraea & $\mathrm{F}$ & 8 & . & . & . & . & . & . & . & $\mathrm{r}$ & $\mathrm{r}$ & . & . & . & . & . & . & . & . & . & . & . & . & . & . & . & . \\
\hline Cladonia novochlorophaea & $\mathrm{F}$ & 8 & . & & . & . & . & . & & $\mathrm{r}$ & $\mathrm{r}$ & . & . & . & . & & & & . & . & . & . & . & . & & . & \\
\hline
\end{tabular}




\begin{tabular}{|c|c|c|c|c|c|c|c|c|c|c|c|c|c|c|c|c|c|c|c|c|c|c|c|c|c|c|}
\hline \multirow[b]{3}{*}{ Aufnahmenummer } & \multirow{3}{*}{ 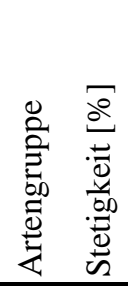 } & \multicolumn{25}{|c|}{ B1 - Cladonio symphycarpae-Sedetum albi } \\
\hline & & \multicolumn{14}{|c|}{ B1a } & \multicolumn{11}{|c|}{ B1b } \\
\hline & & N01 & $\mathrm{N} 02$ & $\mathrm{X} 30$ & $\mathrm{X} 23$ & $\mathrm{X} 42$ & $\mathrm{X} 41$ & $\mathrm{X} 43$ & X39 & $\mathrm{X} 40$ & $\mathrm{X} 45$ & P04 & P05 & $\mathrm{X} 12$ & $\mathrm{X} 77$ & T14 & K01 & X67 & V07 & T04 & L10 & $\mathrm{X} 24$ & $\mathrm{X} 25$ & Y01 1 & K02 & K03 \\
\hline Gesamtartenzahl & & 26 & 22 & 27 & 24 & 42 & 39 & 33 & 45 & 43 & 36 & 34 & 32 & 35 & 32 & 21 & 33 & 24 & 28 & 21 & 45 & 39 & 20 & 41 & 16 & 30 \\
\hline Artenzahl Gefäßpflanzen & & 18 & 15 & 21 & 14 & 26 & 24 & 20 & 19 & 23 & 16 & 24 & 21 & 19 & 19 & 18 & 20 & 15 & 11 & 13 & 35 & 17 & 10 & 20 & 11 & 13 \\
\hline Artenzahl Moose & & 7 & 6 & 6 & 8 & 11 & 10 & 7 & 9 & 8 & 8 & 5 & 5 & 12 & 8 & 3 & 9 & 4 & 5 & 7 & 8 & 10 & 6 & 13 & 4 & 8 \\
\hline Artenzahl Flechten & & 1 & 1 & 0 & 2 & 5 & 5 & 6 & 16 & 11 & 11 & 4 & 6 & 4 & 5 & 0 & 4 & 5 & 12 & 0 & 2 & 12 & 4 & 8 & 0 & 9 \\
\hline
\end{tabular}

\section{Außerdem kommen je einmal vor:}

Gefäßpflanzen: Agrostis capillaris X42: r; Alchemilla glaucescens L10: +; Allium oleraceum X67: r; Androsace septentrionalis X24: 1; Aquilegia vulgaris L10: 1; Berteroa incana X30: r; Capsella bursa-pastoris N02: r; Carex flacca L10: +; Carex muricata X42: r; Carlina spec. L10: +; Chenopodium album X45: r; Convolvulus arvensis X45: r; Euphrasia stricta P05: r; Festuca arundinacea N01: +; Festuca nigescens X77: r; Filipendula vulgaris X12: r; Geranium robertianum ssp. robertianum K03: r; Helictotrichon pratense X12: +; Heracleum sphondylium ssp. sibiricum Y01: r; Hieracium cf. brachiatum L10: +; Hieracium cf. iseranum L10: +; Hieracium subgen. Pilosella Y01: 1; Inula salicina T04: +; Juniperus communis ssp. communis (juv.) V07: r; Lapsana communis V07: r; Leontodo hispidus L10: r; Luzula pilosa L10: r; Melica nutans L10: r; Melilotus albus N02: r; Mycelis muralis X25: r; Myosurus minimus K02: A; Oenothera rubricaulis X30: $\mathrm{r}$; Origanum vulgare ssp. vulgare L10: 1; Phleum phleoides X12: r; Plantago major ssp. major X30: r; Plantago maritima P05: r; Potentilla incana X30: $\mathrm{r}$; Primula veris L10: $\mathrm{r}$; Ranunculus nemorosus L10: $\mathrm{r}$; Ranunculus

polyanthemoides Y01:r; Ranunculus polyanthemos L10: 1; Ribes alpinum V07: r; Rubus caesius N01: r; Scabiosa columbaria X77: r; Silene vulgaris ssp. vulgaris X41: r; Stellaria graminea T14: r; polyanthemoides Y01:1, Ranila Thymus serpyllum P05: +, Thollum pratense P04. I, Tripleurospermum perforatum X45: , Tussilagofarfara L10: r, Veronica verna X30. r, Viola arvensis ssp. arvensis X12: r; Epigäische Moose: P05: r; Riccia bifurca T04: r; Scleropodium purum X41: r; Thuidium philibertii X42: r; Tortula calcicolens X77: A; Weissia brachycarpa X24: r; Epilithische Moose: Orthotrichum spec. K03: r; P05: r; Riccia bifurca T04: r; Scleropodium purum X41: r; Thuidium philibertii X42: r; Tortula calcicolens X77: A; Weissia brachycarpa X24: r; Epilithische Moose: Orthotrichum spec. K03: r; Epigäische Flechten: Cladonia coniocraea X40. I, Cladonia glanca X40. 1, Cladonia phyllophora X40. 1, Cladonia subrangiformis X77. r, Collema bachmanianum X24: r, Collema crispum X12: r; Evernia prunastri X45: r; Leptogium gelatinosum X12: +; Parmelia sulcata X45: r; Peltigera didactyla X39. 1, Pelligera neckeri X42. 1, Ramalina fastigiata X45: r; Epilithische Flechten: Acarospora glaucocarpa X39: r; Aspicilia contorta ssp. contorta V07: r; Caloplaca lithophila X67: r; Candelariella aurella K03: r; Catillaria chalybeia Y01: r; Cladonia coniocraea X40: r; Lecanora polytropa X39: r; Lecidella carpathica X39. r, Lecidella cr. euphorea X39. r, Phaeophysica orbicularis P04. r, Protoparmeliopsis muralis P05: r, Epixyle Fechten: Cetraria sepincola X45: r, Lecanora carpinea X24: Lecanora spec. X45: r; Parmelia sulcata X45: r; Physcia tenella X45: r; Placynthiella icmalea X24: r; Ramalina farinacea X45: r; Rinodina gennarii X24: r; Trapeliopsis granulosa X24: r; Xanthoria candelaria X45: r; Epiphythische Flechten: Cladonia pyxidata agg. V07: r 


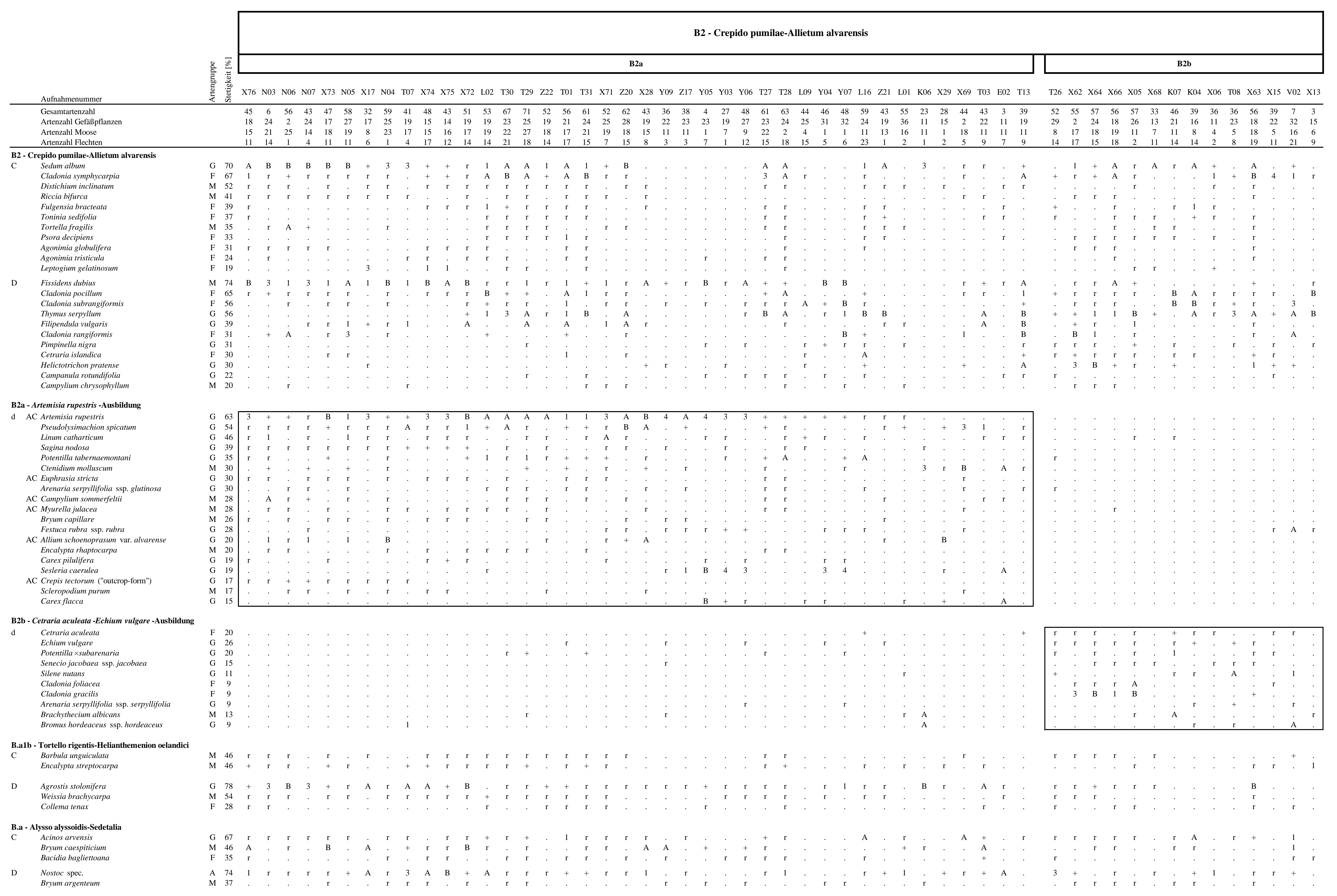




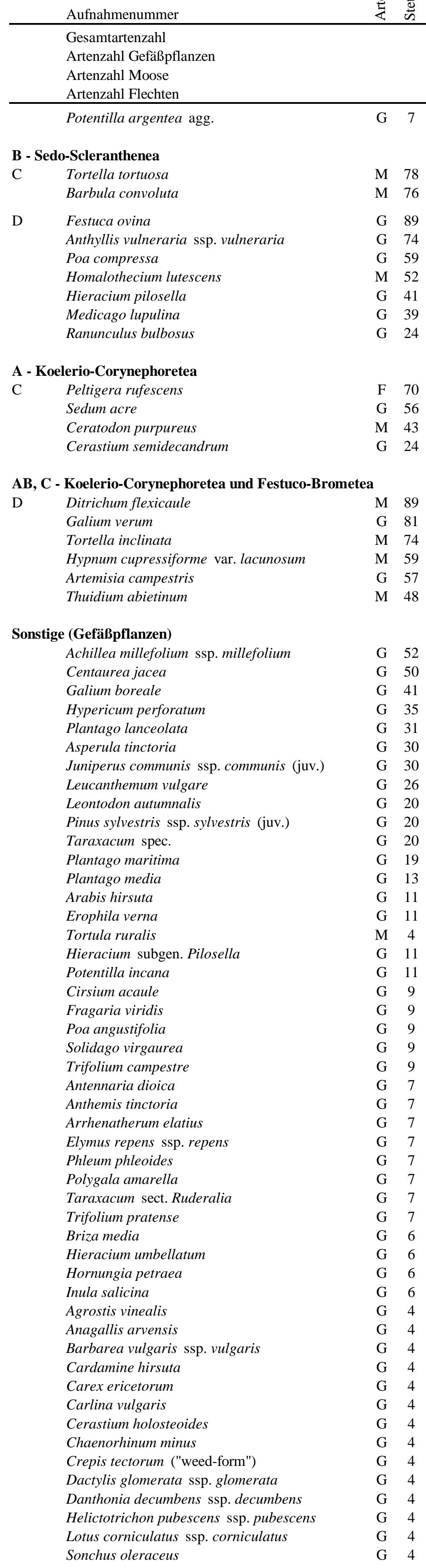

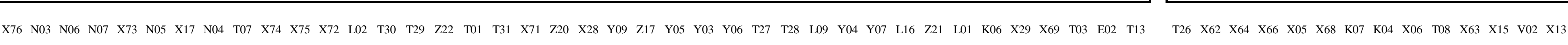

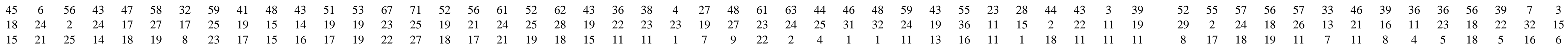

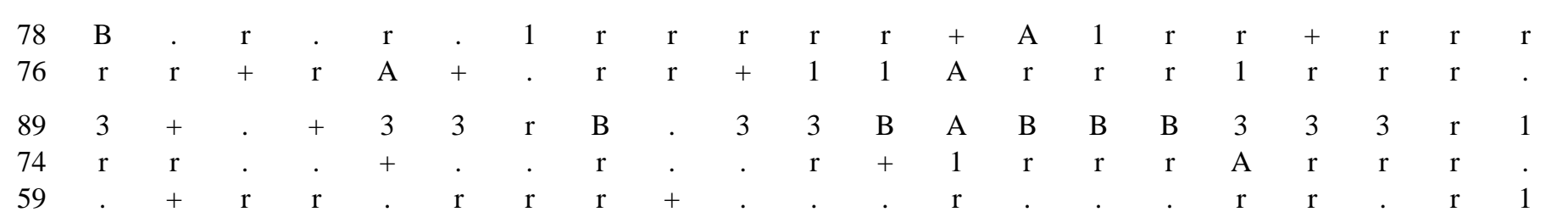




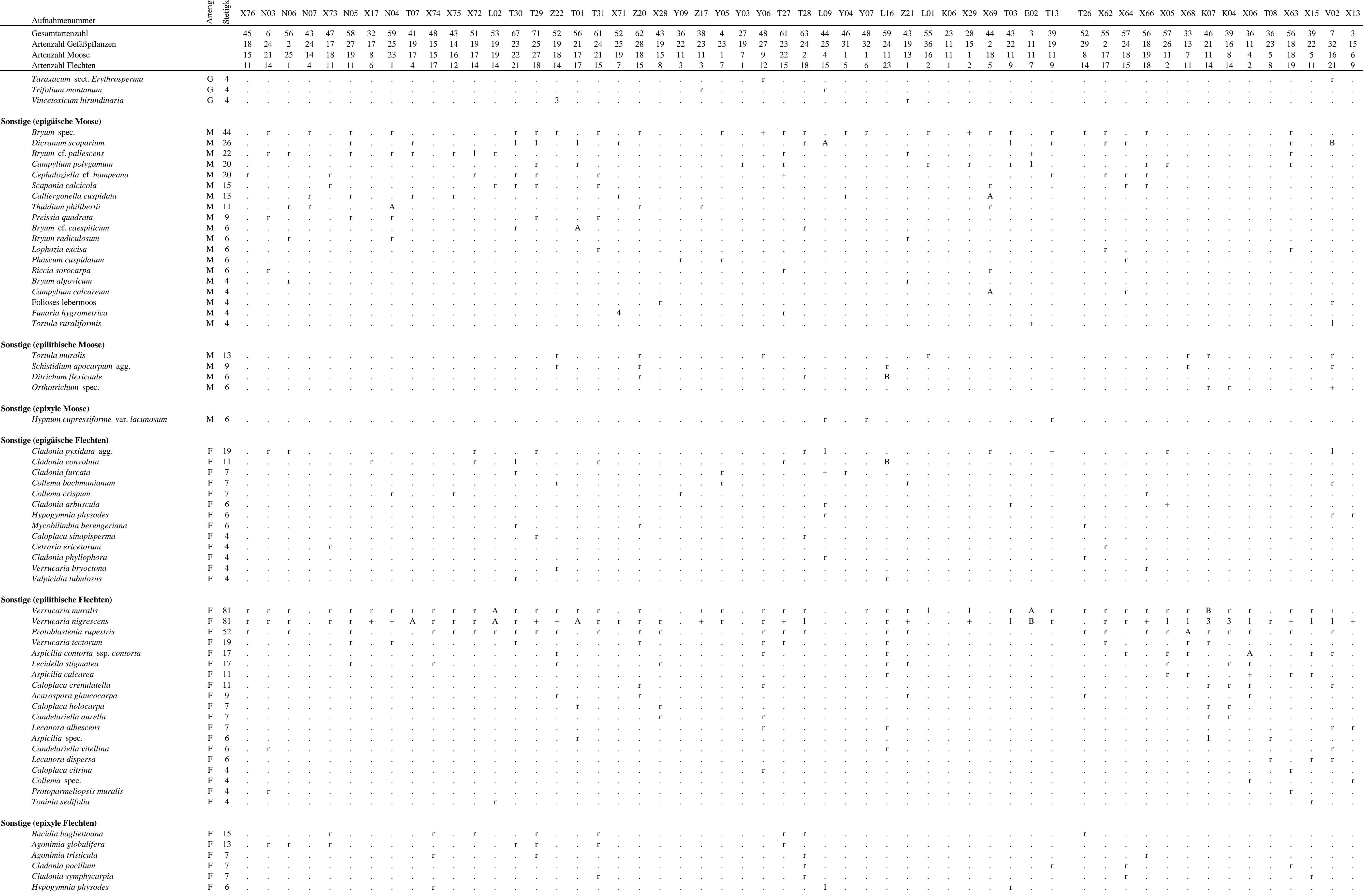




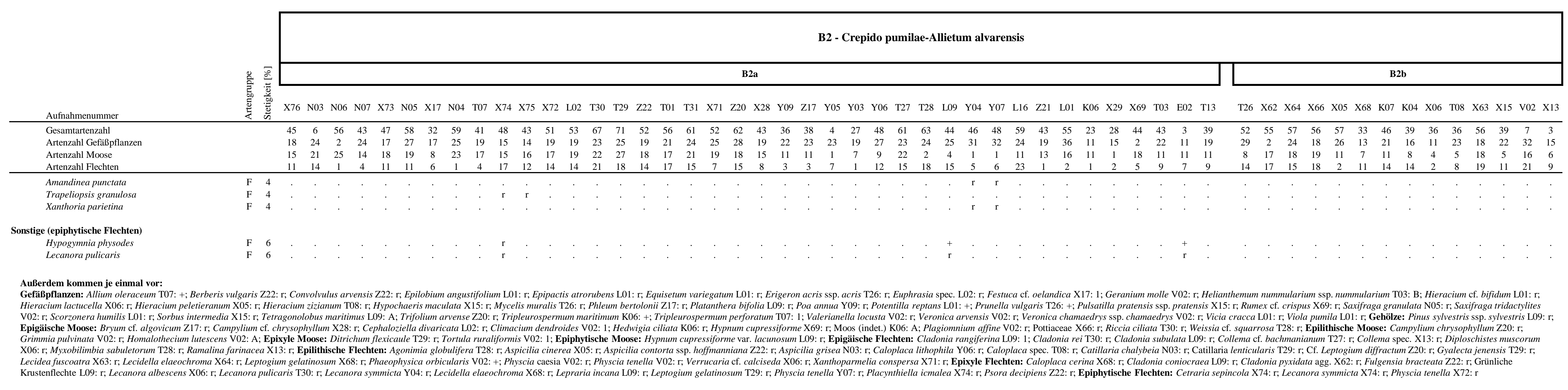




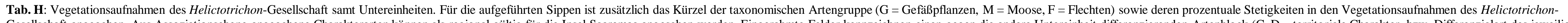
Gesellschaft angegeben. Aus Assoziationsebene angegebene Charakterarten können als regional gültig für die Insel Saaremaa ange
Syntaxons; $\mathrm{d}$ = Differenzialart unterhalb des Assoziationsniveaus). Am Ende der Tabelle sind außerdem die Kopfdaten aufgelistet.

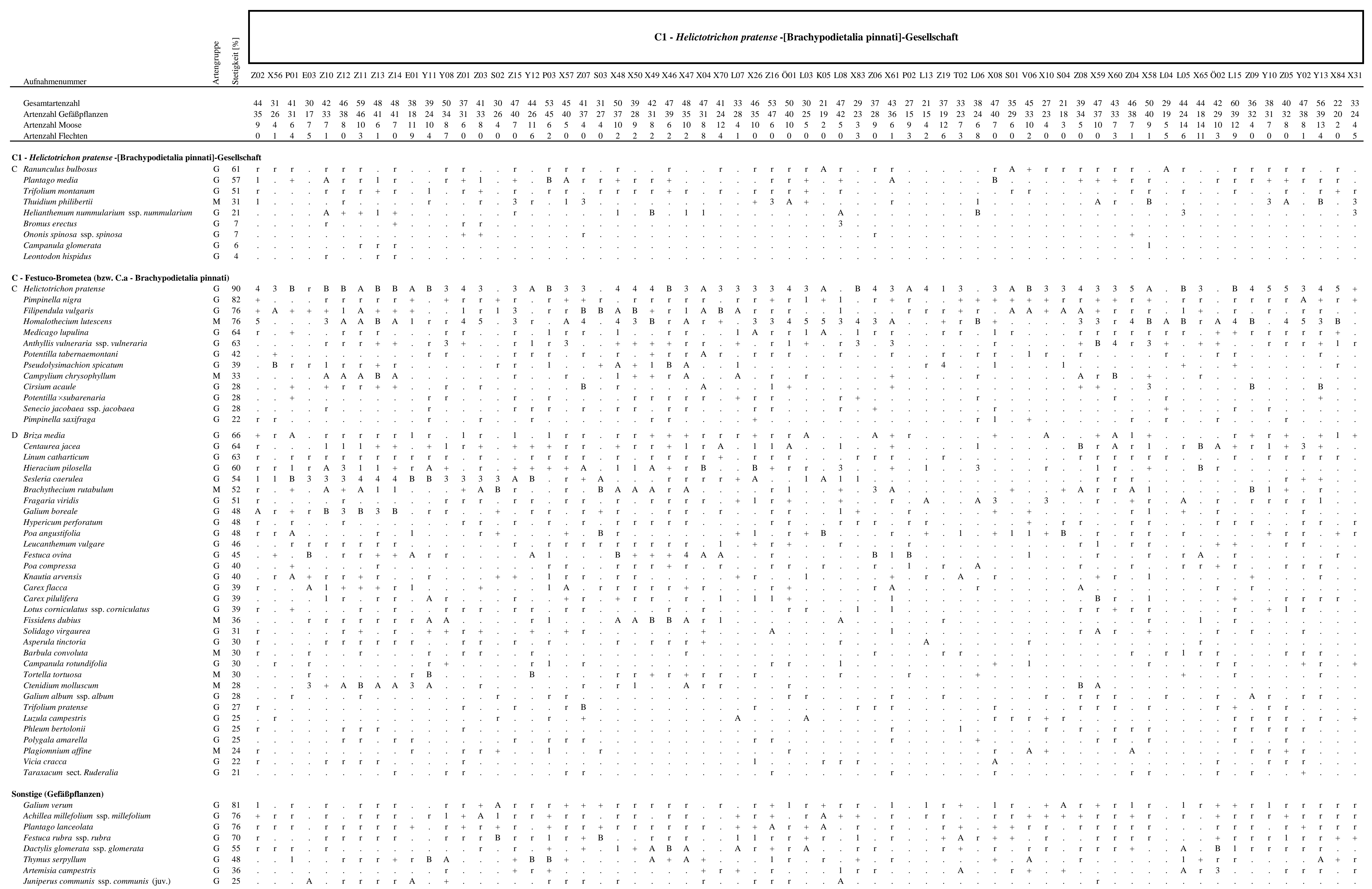




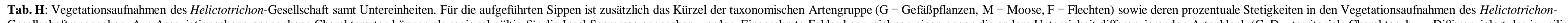

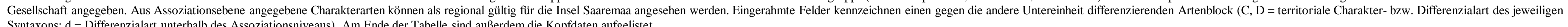
Syntaxons; d = Differenzialart unterhalb des Assoziationsniveaus). Am Ende der Tabelle sind außerdem die Kopfdaten aufgelistet.

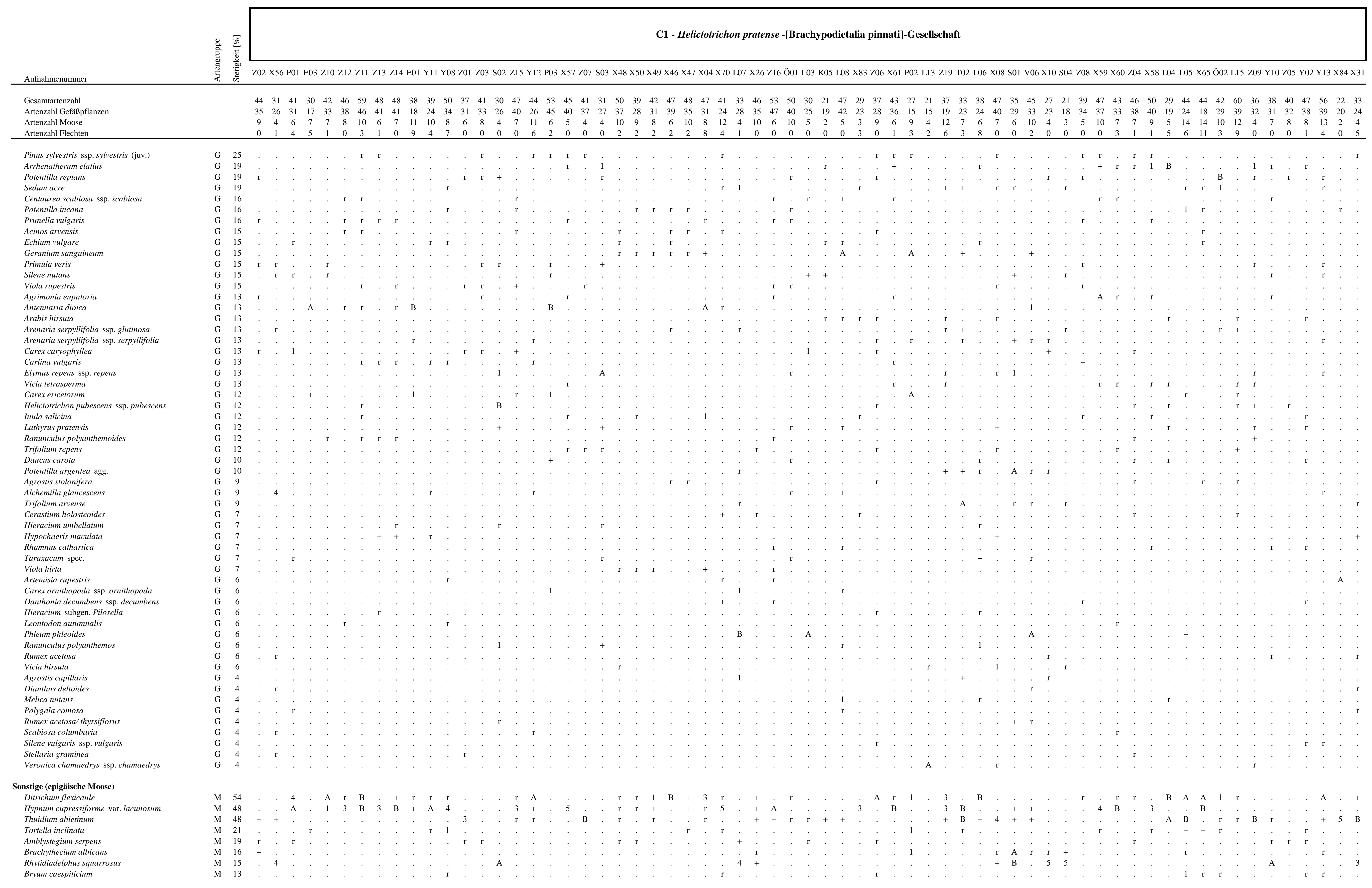




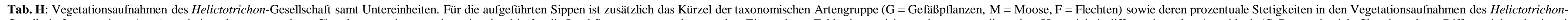
Syntaxons; d $\mathrm{d}$ D Differenzialart unterthalb des Assoziationsniveaus). Am Ende der Tabelle sind außüerdem die Kopfdaten aufgelistet.

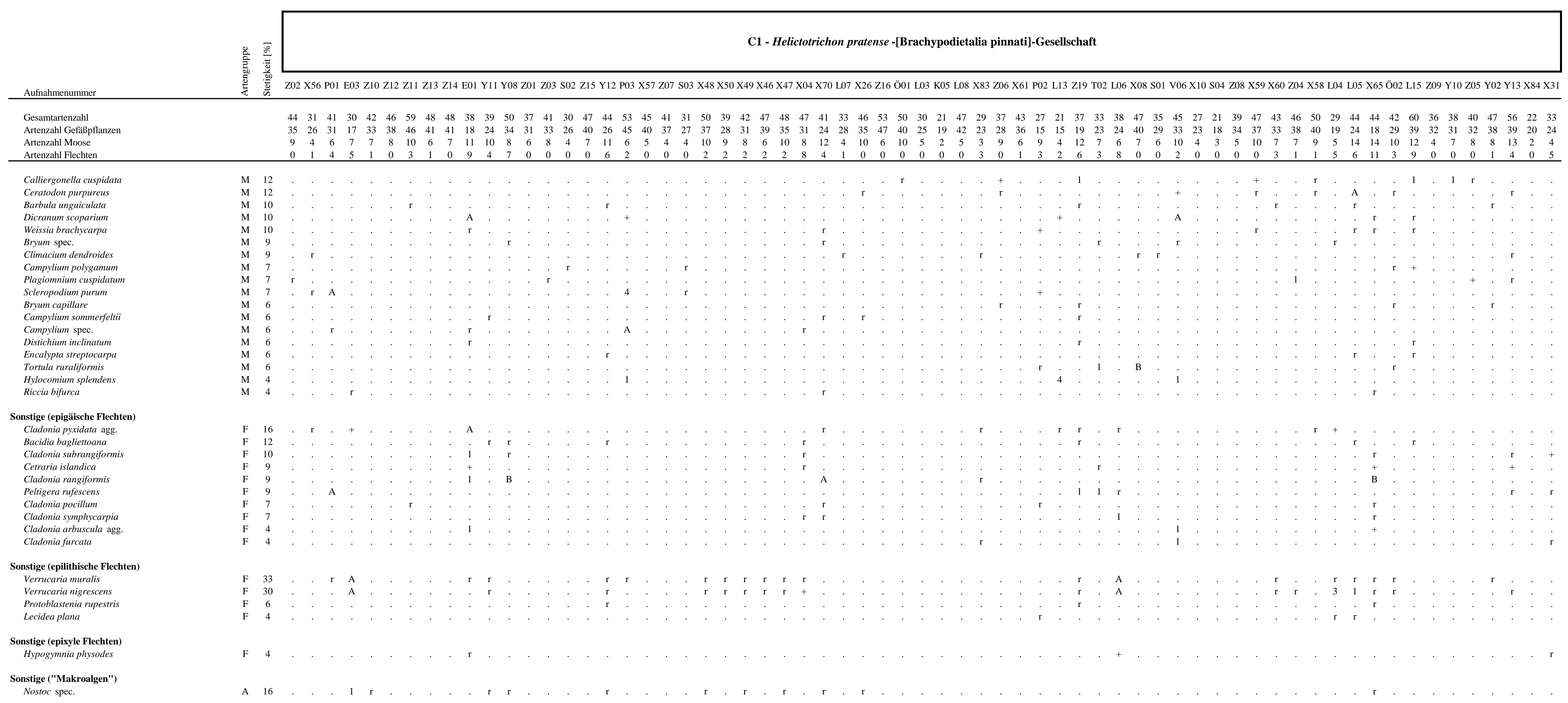

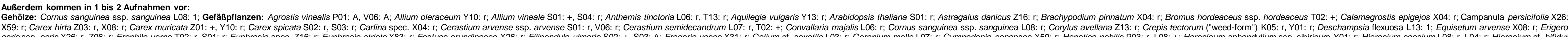

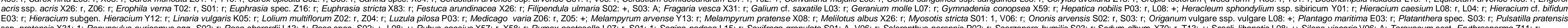

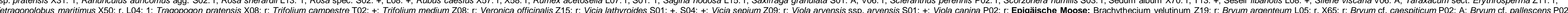

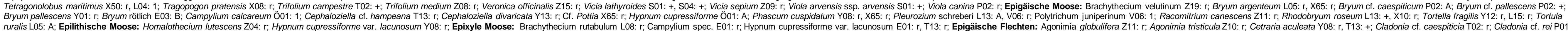

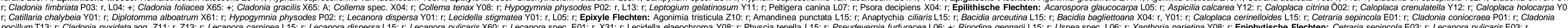

